九州大学学術情報リポジトリ

Kyushu University Institutional Repository

\title{
CHRYSOMELIDAE (COLEOPTERA) OF THAILAND, CAMBODIA, LAOS AND VIETNAM. III. EUMOLPINAE
}

Kimoto, Shinsaku

Gressitt, J. Linsley

https://doi.org/10.5109/2421

出版情報 : ESAKIA. 18，pp.1-141，1982-11-25. Entomological Laboratory，Faculty of Agriculture， Kyushu University

バージョン :

権利関係 : 
ESAKIA， (18): 1-141. 1982

\title{
CHRYSOMELIDAE (COLEOPTERA) OF THAILAND, CAMBODIA, LAOS AND VIETNAM. III. EUMOLPINAE ${ }^{1 / 2) 33}$
}

\author{
SHINSAKU Ki м о т о \\ Biological Laboratory, Department of General Education, \\ School of Medicine, Kurume University, Kurume 830, Japan \\ and \\ + J. Linsley Gressitw ${ }^{4}$ \\ Bishop Museum, Honolulu, Hawaii 96819, U.S. A. and \\ Wau Ecology Institute, Wau, Papua New Guinea
}

\begin{abstract}
This third paper of a series treats the subfamily Eumolpinae covering 207 species in 41 genera. The keys treat 31 genera and 195 species. Relevant synonymies are presented for genera and species, as well as general and local distributions of species. Fourty-five species are described as new and a number of species are newly recorded from the area or from individual countries. Eighty-seven species and 7 genera are relegated to synonymy.
\end{abstract}

This paper is the third in a series" attempting to cover the chrysomelid beetles from the Thai-Indochina area. This series represents a sequel to "Chrysomelidae (Coleopt.) of China and Korea" (Gressitt \& Kimoto, 1961 \& 1963), and should be used in conjunction with that monograph. This installment treats the subfamily Eumolpinae covering 41 genera, 207 species, including 45 new species and 87 new synonymies. Seven genera are synonymized and there are many new combinations.

Keys are presented to genera and species, including a few occurring just outside the area of treatment. Pertinent synonymies are presented, except that references under genera included in Gressitt \& Kimoto (1961) are not

1) Part I, 1979, Pacific Insects, 20 (2-3) : 191-256; Part II, 1981, ibid., 23(3-4): 286-391.

2) Partly supported by a grant from the Japan Society for the Promotion of Science, as part of the Japan-U.S. Cooperative Science Program : Zoogeography and Ecology of Pacific Area Insects.

3) Partial results of research and fieldwork supported by grants to Bishop Museum from the U. S. National Institute of Health (AI-01723), the U. S. Army Medical Research \& Development Command (DA-MEDDH-60-1), and the U. S. National Science Foundation (GF-58, G-2127).

4) Passed away on April 26, 1982 in a plane crash in south China. 
repeated (other than original references and type designations). All known geographical records within the area, as well as general distribution for all the species treated, are also presented. A number of species are newly recorded from this general region, as well as a number of new records to individual countries.

The sources of material are essentially the same as for the preceding installment. The main sources are Bishop Museum collections including the large J. A. Rondon collection from Laos (Gressitt et al., 1970) ; collections from various Japan-United States scientific cooperation projects funded by the U. S. National Science Foundation, the Japan Society for the Promotion of Sciences, and the Ministry of Education of Japan ; results of the rice stem-borer studies under the direction of Prof. K. Yasumatsu and Prof. Y. Hirashima ; collections from Kasetsart University and the Thai Department of Agriculture, Bangkhen; collections of Zoologische Staatsammlung, München, G. Frey Museum, Naturhistorisches Museum, Base1 and Entomological Laboratory, Ehime University; as well as private collection made by Dr. Kintaro Baba and others. For further details refer to the introduction in Kimoto \& Gressitt $(1978,1981)$.

For general information on the principal Laos survey and for locations of collecting localities, see introductory section, map and list of localities of Gressitt et al. (1970).

The following abbreviations are used herein for depository museums:

BANGKHEN Kasetsart University and Agriculture Department, Bangkhen, Thailand.

BASEL Naturhistorishes Museum, Basel.

BISHOP Bishop Museum, Honolulu.

BMNH British Museum (Natural History), London.

BRUXELES Institute Royal des Sciences Naturelles, Bruxelles.

CAS California Academy of Sciences, San Francisco.

CHUJO Dr. Michio Chûjô collection, Shizuoka.

EHIME Ehime University, Matsuyama.

FREY G. Frey Museum, Tutzing bei Mtinchen.

GENOVA Museo Civico di Storia Naturale, Genova.

HAMBURG Universitat Hamburg, Zoologisches Institute und Zoologisches Museum, Hambrug.

KIMOTO S. Kimoto collection, Kurume.

KØBENHAVN University Zoological Museum, Kфbenhavn (Copenhagen).

KU Kyushu University, Fukuoka.

MCZ Museum of Comparative Zoology, Harvard University, Cambridge, Massachusetts.

OHNO Masao Ohno collection, Saitama,

MUNCHEN Zoologische Staatsammlung, Mtinchen.

PARIS Museum National d'Histoire Naturelle, Paris. 
STOCKHOLM Naturhistoriska Riksmuseum, Stockholm.

TAR1 Taiwan Agriculture Research Institute and Taiwan University, Taipei.

ZMB Zoologisches Museum der Humboldt-Universitat zu Berlin.

The abbreviation "Umgeb." in geographical records refers to the German "Umgebung", meaning "Environs of".

In the course of the study we are grateful to authorities and staff members at Bishop Museum, Honolulu ; British Museum (Natural History), London ; Museum National d'Histoire Naturelle, Paris; Museo Civico di Storia Naturale, Genova; Zoologische Staatsammlung, Miinchen ; G. Frey Museum, Tutzing bei München; Kasetsart University and Agriculture Department, Bangkhen ; Kyushu University, Fukuoka ; Ehime University, Matsuyama ; California Academy of Sciences, San Francisco; National Museum of Natural History, Washington DC. Also, we are especially obliged to Drs. R. D. Pope, A. Viliers, Nicole Berti, G. Scherer, R. L. A. Damoiseau, R. Poggi, W. Wittmer, G. A. Samuelson, H. B. Leech, D. Kavanaugh, P. Arnaud, K. Yasumatsu, Y. Hirashima, S. Asahina, T. Ishihara, K. Baba, the late J. A. Rondon, and Mrs. Carol N. Higa.

\section{K EY TO GENERA OF EUMOLPINAE}

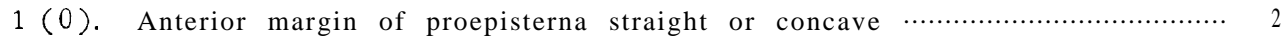

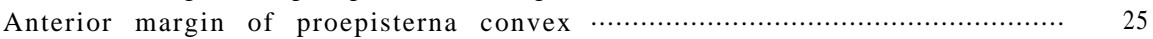

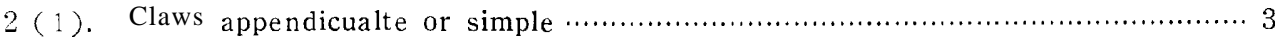

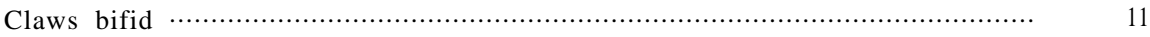

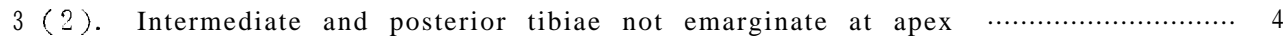

Intermediate and posterior tibiae, or intermdiate tibia emarginate at apex $\ldots . .7$

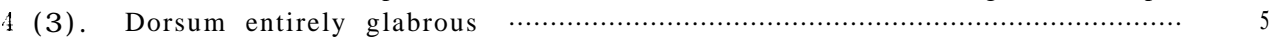

Dorsum densely covered with erect hairs ........................... Paracrothinium

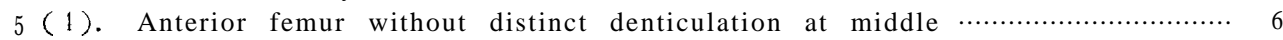

Anterior femur with a distinct denticulation at middle ............. Chrysolampra

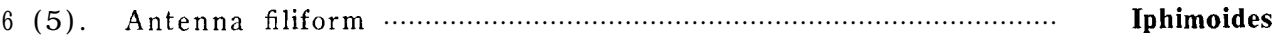

Antenna robust, flattened, attenuated towards apex .................. Clisitherella

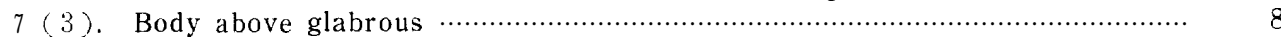

Body above thickly covered with pubescence ….......................... Parascela

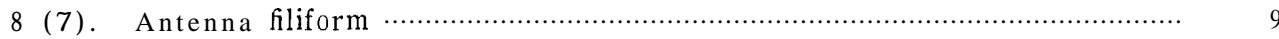

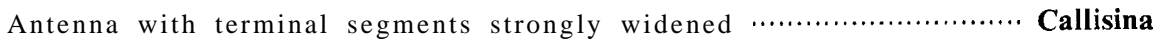

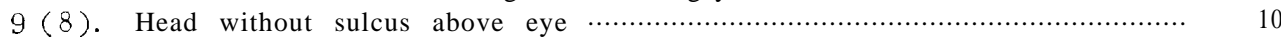

Head with sulcus above eye ….............................................. Pagria

10 (9). Prothorax as broad as elytron; body generally very small and rounded; antenna robust, nearly as long as in preapical antennal segments, and

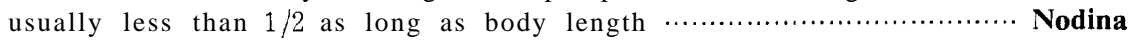

Prothorax narrower than elytron at base; antenna slender, long, and often more than $2 / 3$ as long as body length ......................... Basilepta

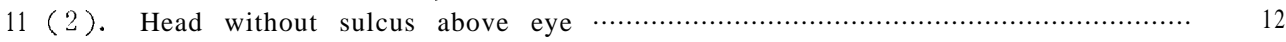

Head with deep sulcus above eye ….................................... Scelodonta

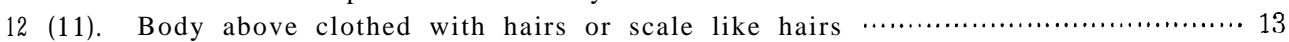

Body above glabrous …................................................ Colasposoma 

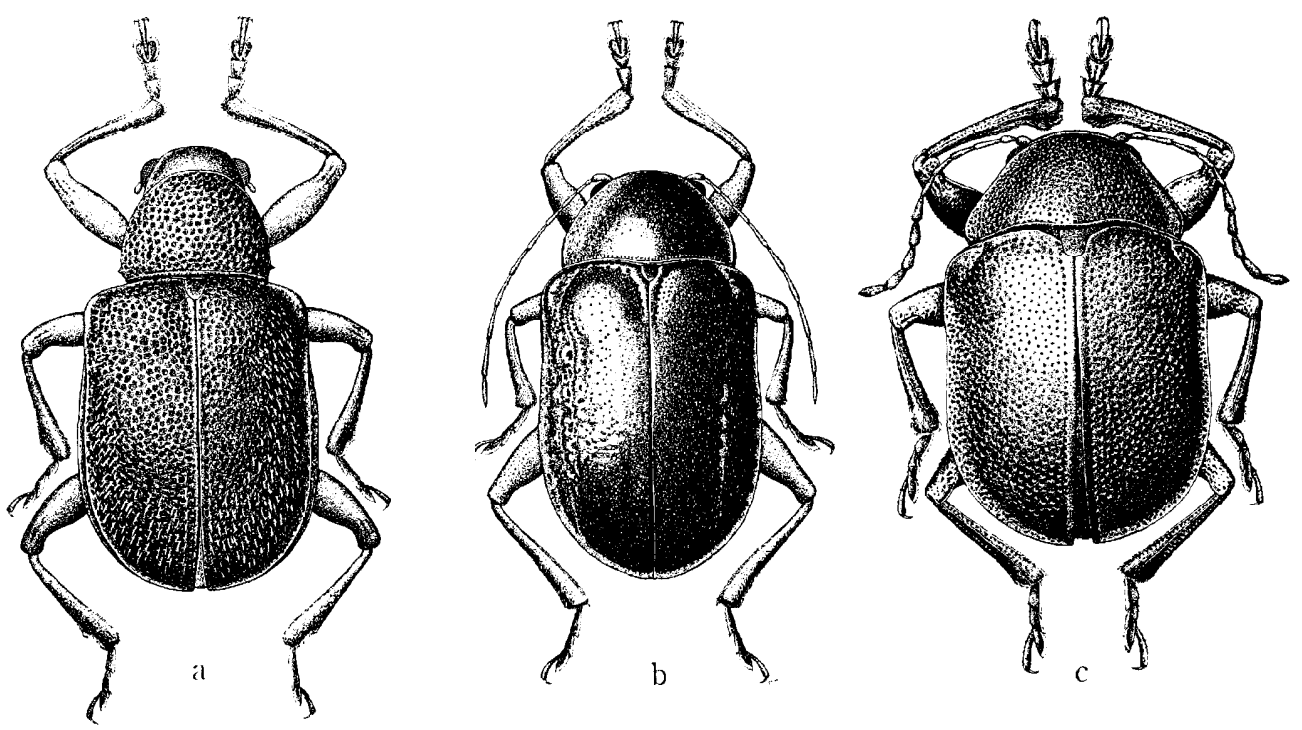

Fig. 1. a, Parascela cribrata ( $\mathrm{Schaufuss)} \mathrm{;} \mathrm{b,} \mathrm{Chrysolampra} \mathrm{splendens} \mathrm{Baly} \mathrm{;} \mathrm{c,} \mathrm{Colasposoma}$ downesii Baly.

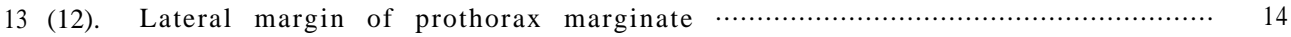

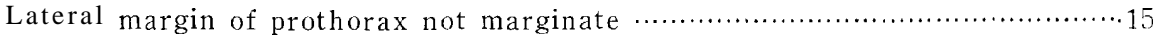

14 (13). Intermediate and posterior tibiae not emarginate at apex ............... Osnaparis Intermediate and posterior tibiae, or intermediate tibia emarginate at apex

Trichochrysea

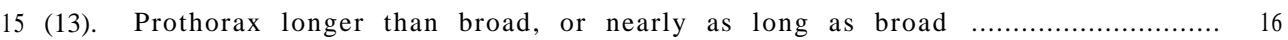

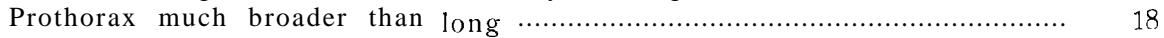

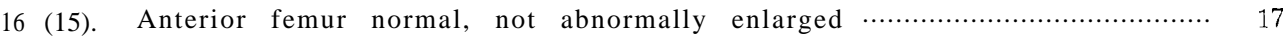

Anterior femur abnormally enlarged …..................................... Piomera

17 (16). Femora unarmed beneath ; body small, metallic ............................... Malegia

Femora distinctly toothed beneath …................................ Lypesthes

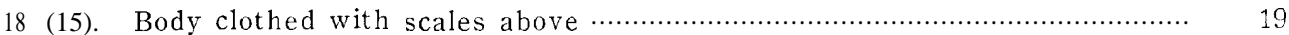

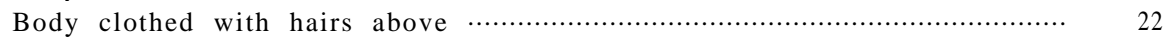

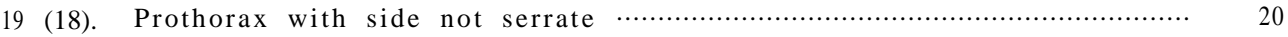

Prothorax with side entirely serrate .................................. Pseudometaxis

20 (19). Anterior and posterior femora not thicker than intermediate one; pos-

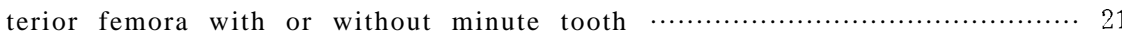

Anterior and posterior femora thickened, intermediate femur more slenderer; posterior femur, in some case anterior femur also, each with a

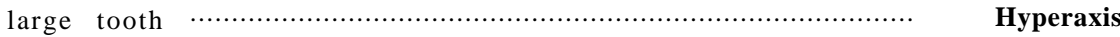

21 (20). Dorsum covered with long, strongly curved, suberect scales which are narrowed and thread-like apically

Hemiplatys Dorsum covered with normal scales which are usually depressed ........ Demotina

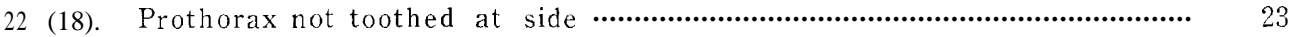

Prothorax with two or three fairly prominent teeth at side .................... 24

23 (22). Mesosternum transverse, more or less broadly emarginate apically ; generally longer than $4 \mathrm{~mm}$

Aoria

Mesosternum oblong or subquadrate; generally 2-3 $\mathrm{mm}$ in length in this fauna 
24 (22). Interantennal space deeply excavated ; body length over $10 \mathrm{~mm}$...... Goniopleura Interantennal space generally convex; body length nearly $3-4 \mathrm{~mm}$........ Aulexis

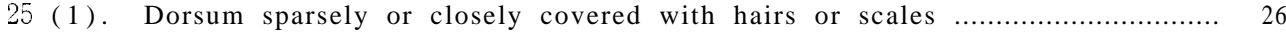

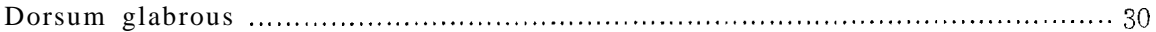

26 (25). Dorsum without transverse rugosities, closely covered with hairs or scales

Dorsum with strong transverse rugosities and short, adpressed sparse pubescence

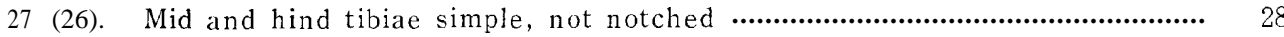

Mid and hind tibiae notched on outer side near apex; dorsum covered with scales

\section{Pachnephorus}

28 (27). Tarsal claws appendiculate ; dorsum covered with hairs .......................... 29

Tarsal claws bifid; dorsum covered with large, broad, rigid, adpressed or suberect curved scales; prosternum with a deep groove for reception of antenna

Aulacolepis

29 (28). Prosternum without groove for reception of antenna; antenna subfiliform, not broadly dilated; femora without distinct tooth beneath ...... Lophea

Prosternum with grove at side for reception of antenna; antenna very broadly dilated at apical segments; femora with a distinct tooth beneath

Heterotrichus

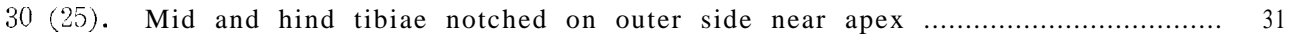

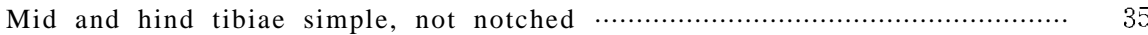

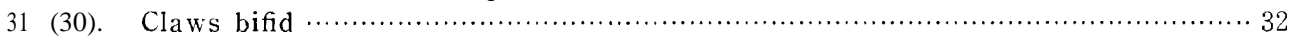

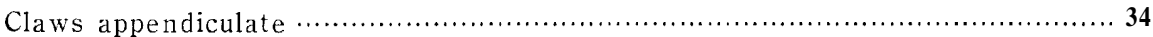

32 (31). Fore femur with large tooth beneath; head without a deep groove above eye

Fore femur unarmed or minutely toothed beneath; head with a deep groove above eye and groove large and deeply emarginate

33 (32). Elytron dilated posteriorly and with epipleuron convex

Elytron subparallel-sided and with epipleuron flat

34 (31). Antenna filiform, with terminal segments widened
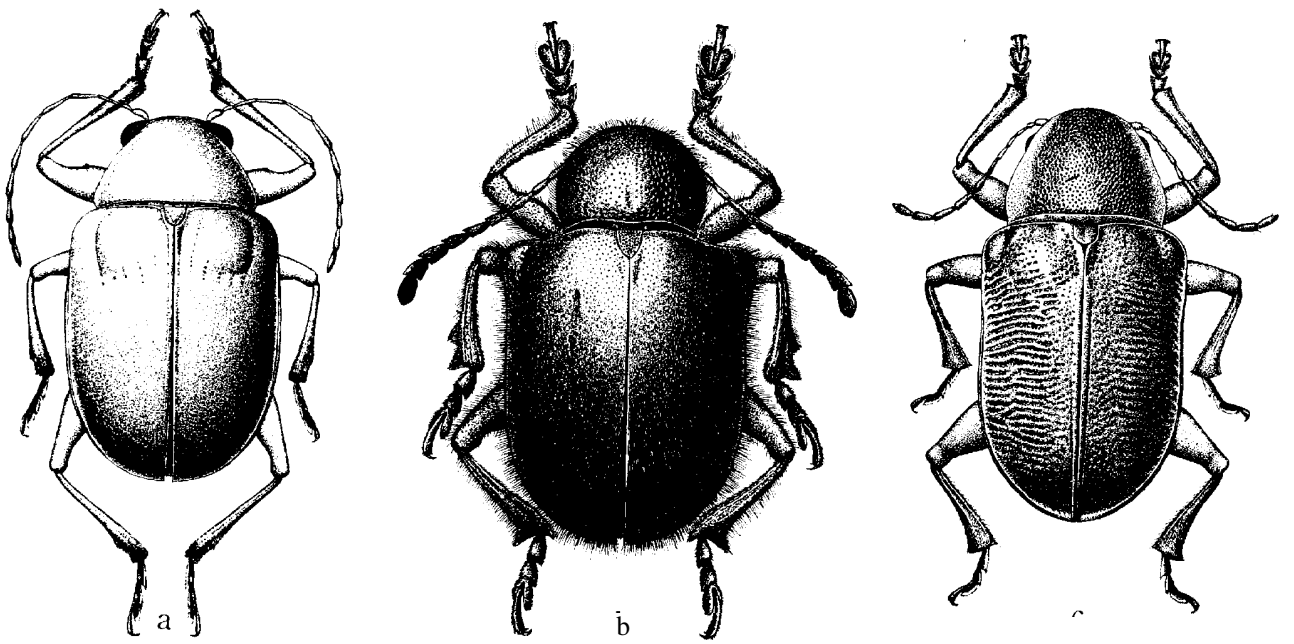

Fig. 2. a, Basilepta beccarii (Jacoby); b, Trichochrysea imperialis (Baly) ; c, Abirus fortuneii (Baly). 
Antenna with four basal segments cylindrical, following segments compressed feebly dilated

35 (30). Head without sulcus above eye

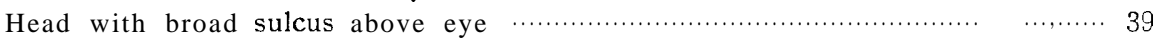

36 (35). Prothorax subquadrate or cylindrical, distinctly narrower at base than

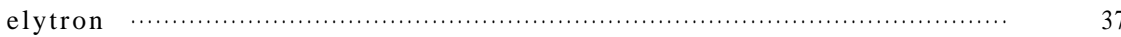

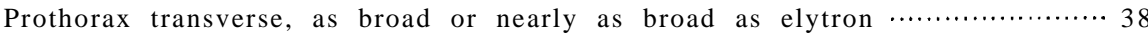

37 (36). Prothorax cylindrical, subparallel-sided, lateral margin not visible from abvoe …....................................................... Chalcolema

Prothorax subquadrate, widest slightly before middle, narrowed anteriorly and posteriorly; lateral margin distinct, visible from above ….. Heminodes

38 (36). Posterior femur with long projection subapically in male, and with distinct spine in female ..................................................... Olorus

Posterior femur sometimes with distinct spine subapically .............. Colaspoides

39 (35). Sulcus above eye deep and broad, antenna with apical segments widened and flattened Platycorynus

Sulcus above eye much shallower and not so broad as in Platycorynus; antenna with apical segments either cylindrical or not distinctly flattened

Chrysochus

\section{Genus Paracrothinium Chen}

Paracrothinium Chen, 1940, Sinensia 11 (3-4) : 209 (type : Paracrothinium cupricolle Chen, from China) ; 1940, ibid. 11 (5-6) : 489. -Gressitt \& Kimoto, 1961, Pac. Insects Monogr. 1A: 233.

\section{KeY TO SPECIES OF Paracrothinium}

$1(0)$. Head and pronotum reddish, elytron bluish 2

Head reddish brown, prontoum pitchy black with anterior margin brownish, scutellum reddish brown, elytron reddish brown with lateral margin blackish in basal portion; ventral surfaces pitchy brown; antenna pitchy black with four or five basal segments brownish; legs pitchy black with basal portions of femora brownish ; length 8.0-9.0

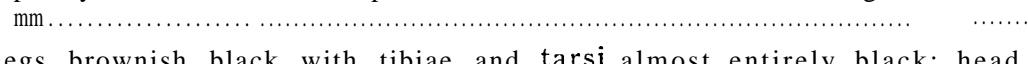

2(1). Legs brownish black with tibiae and tarsi almost entirely black; head greenish cupreous; ventral surfaces metallic; antenna black with four

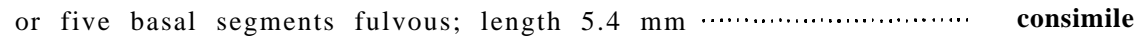

Legs reddish brown with tibiae, tarsi and apical portions of femora black; head, pronotum and scutellum deep red; elytron violaceous blue, ventral surfaces entirely reddish brown; antenna black with four basal

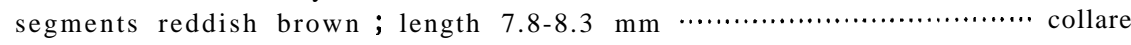

\section{Paracrothinium collare Kimoto \& Gressitt, n. sp. (Fig. 4a)}

Oblong oval ; head, prontoum and scutellum deep red; elytron violaceous blue; antenna black with four basal segments reddish brown; ventral surfaces entirely reddish brown; legs reddish brown with tibiae, tarsi and apical portions of femora black.

Head with surface distinctly but not closely punctate and interstices of 
punctures smooth, shining, and sparsely covered by fine hairs especially on lateral portion ; clypeus distinctly raised, vertex slightly depressed at anterior portion and with a shallow longitudinal furrow at middle. Antenna robust, depressed, nearly $3 / 4$ as long as body length; first segment robust, somewhat club-shaped; second nearly $2 / 3$ as long as first in length, robust; third nearly $11 / 2$ times as long as second, slender; fourth subequal to third in length and shape; fifth slightly longer than fourth ; sixth subequal to fifth in length but wider in apical portion, subtriangular ; seventh nearly as long as sixth but much robuster; eighth slightly shorter than seventh and nearly $11 / 4$ times as long as wide; ninth and tenth subequal to eighth in length and shape; eleventh about $1 \frac{1 / 2}{2}$ times as long as tenth and its apex pointed. Pronotum transverse, about twice as wide as long, side rounded, widest slightly behind middle and strongly narrowed anteriorly and less strongly so posteriorly; anterior and posterior corners each with a setigerous puncture; anterior margin nearly straight, posterior margin widely rounded posteriorly and slightly produced at middle; dorsal surface sparsely covered with fine hairs and distinctly but not closely punctate and their interstices smooth, shining, nearly impunctate; pro- epimeron slightly wrinkled, nearly impunctate ; metasternum smooth, shining, sparsely covered by fine hairs and sparsely impressed by small punctures. Scutellum subpentagonal, with apex rounded ; surface sparsely impressed by minute punctures. Elytron slightly rounded at side, without distinct subbasal transverse furrow; surface entirely covered with fine hairs and closely and distinctly punctate, and their interstices smooth, shining.

Length 7.8-8.3 $\mathrm{mm}$.

H olotype (BISHOP), Laos: Ban Van Heue, 20 km E of Phou-kow-kuei, 1531. v. 1965, J. A. Rondon. Paratype: Laos: Umgeb. Vientiane, 1 ex., iii-vi. 1963 (MUNCHEN).

This new species somewhat resembles $\boldsymbol{P}$. consimile Chen, but differs in being the body length larger and having the ventral surface entirely reddish brown.

\section{Paracrothinium consimile Chen}

Paracrothinium consimile Chen, 1940, Sinenšia 11: 210 (Tonkin).

DistRIBUTION : Vietnam.

No additional material.

\section{Paracrothinium latum(Pic) New combination}

Iphimoides latus Pic, 1928, Bull. Soc.Zool. Fr. 53: 378 (Tonkin; PARIS).

Distribution: Vietnam.

No additional material. 


\section{Genus Chrysolampra Baly}

Chrysolampra Baly, 1859, Ann. Mag. Nat. Hist. ser. 3, 4: 126 (type : Chrysolampra splendens Baly, China). -Chapuis, 1874, Genera Coleopt. 10: 237. -Lefèvre, 1885, Mém. Soc. Sci. Liège, ser. 2, 11: 12. -Jacoby, 1908, Fauna India, Coleopt. 2: 285. -Chen, 1935, Sinensia 6(3): $243 ; 1940$, ibid. 11(5-6) : 492. -Gressitt \& Kimoto, 1961, Pac. Insects Monogr. $1 \mathrm{~A}: 284$.

\section{K EY TO SPECIES OF Chrysolampra}

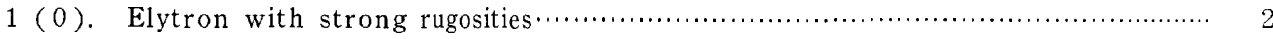

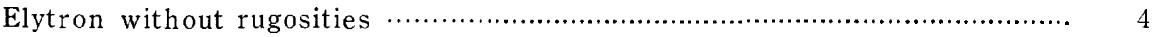

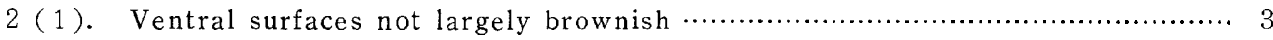

Ventral surfaces reddish brown, with meta-epimeron greenish black; head, pronotum and elytron cupreous with greenish luster; length 9

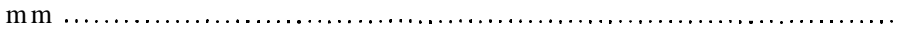

rufimembris

3 (2). Pronotum with anterolateral portion sparsely covered with fine but distinct hairs, lateral margin of pronotum nearly straight, and widely marginate, especially on basal portion ; general color bluish black, bluish green, with or without cupreous luster; antenna reddish brown to pitchy brown; rugosities of elytron transverse, not very strongly

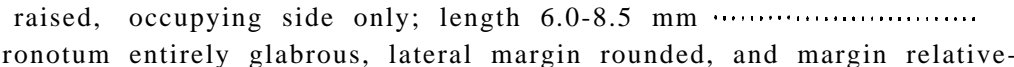
mouhoti ly narrower; rugosities of elytron more or less rounded, tubercular, generally strong and prominent; bluish black, blue, bluish green, violaceous or cupreous ; antenna reddish brown to pitchy black; length

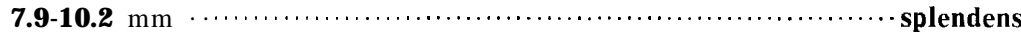

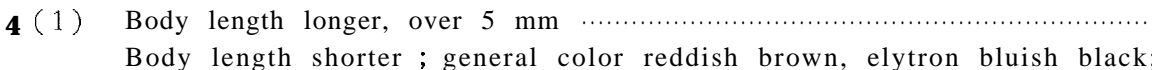
ventral surfaces of meso- and metathorax and abdomen partly blackish in various degrees; antenna and legs entirely reddish brown; length 3. 2-3. $4 \mathrm{~mm}$.

minuta

5 (4) Metasternum with surface sligtly wrinkled and sparsely covered with fine hairs; head and pronotum cupreous ; elytron violaceous blue; underside obscure blue, antenna pitchy black with four basal segments fulvous ; length 5.3-8.0 $\mathrm{mm}$

cuprithorax

Metasternum with surface finely granulate and closely covered with fine hairs; dorsal surfaces violaceous, bluish black, bluish green or cupreous green; antenna reddish brown; length 6.9-9.1 $\mathrm{mm}$

laosensis

\section{Chrysolampra cuprithorax Chen}

Chrysolampra cuprit horax C hen, 1935, Sinensia 6: 247 (Tonkin ; PARIS). - Gressitt \& Kimoto, 1961, Pac. Insects Monogr. 1A: 284 (Tonkin).

Distribution : Vietnam.

M A T E R A L EXAM E D. VIETNAM: Hoa-Binh, Tonkin, 5 exs., A. Cooman (FREY). 
Chrysolampra laosensis Kimoto \& Gressitt, n. sp. (Fig. 3a)

Oblong oval ; general color violaceous, bluish black, bluish green or cupreous green, antenna reddish brown.

Head with surface strongly and rather closely punctate and interstices of punctures finely shagreened, vertex sparsely covered with fine hairs and with a shallow longitudinal furrow at middle and a disticnt tubercle at middle of anterior part. Antenna slender, filiform, nearly $2 / 3$ as long as body length; first segment robust, somewhat club-shaped ; second nearly half as long as first, and nearly $2 / 3$ as long as wide, robust; third nearly $21 / 5$ times as long as second, slender; fourth subequal to third in length and shape; fifth nearly $11 / 4$ times as long as fourth ; sixth slightly longer than fifth; seventh subequal to sixth in length and shape; eighth distinctly shorter than seventh; eighth to tenth subequal to each other in length and shape; eleventh subequal to tenth in length but its apex pointed. Pronotum transverse, about twice as wide as long, side rounded, widest slightly before basal margin and strongly narrowed anteriorly; anterior and posterior corners each with a setigerous puncture; anterior margin rounded, produced anteriorly at middle and posterior margin widely rounded posteriorly; dorsal surface distinctly but not closely punctate and their interstices sparsely impressed by fine punctures; proepimeron wrinkled, nearly impunctate ; metasternum finely granulate, impressed by large punctures and covered by fine hairs. Scutellum subpentagonal, with apex rounded; surface smooth, shining, sparsely impressed by minute punctures. Elytron slightly rounded at side, subbasal area separated by subbasal transverse furrow which is obsolete at middle and distinctly impressed at lateral part; surface closely and distinctly punctate, and their interstices slightly raised on lateral part.

Length $6.9-9.1 \mathrm{~mm}$.

Holotype (BISHOP), LAOS : Vientiane Prov., Ban Van Eue, 15. v. 1965, Native collr. Paratopotype: 1 ex., same data as the holotype (KIMOTO). Paratypes : LAOS: Ban Van Eue, $20 \mathrm{~km}$ E of Phou-kow-kuei, 3 exs., 15-31. v. 1965, J. A. Rondon (BISHOP) ; Umg. Vientiane, 3 exs., iii-vi. 1963; Umgeb. Paklay, 1 ex., 1963 (MUNCHEN).

This new species closely resembles Chrysolampracuprithorax Chen, but differs in having the surface of metasternum finely granulate and more closely covered by fine hairs.

Chrysolampra minuta Kimoto \& Gressitt, n. sp. (Fig. 3c)

Oval; general color reddish brown, elytron bluish black; ventral surfaces of meso- and metathorax and abdomen partly blackish in various degrees; antenna and legs entirely reddish brown.

Head with surface distinctly and not closely punctate and interstices of 

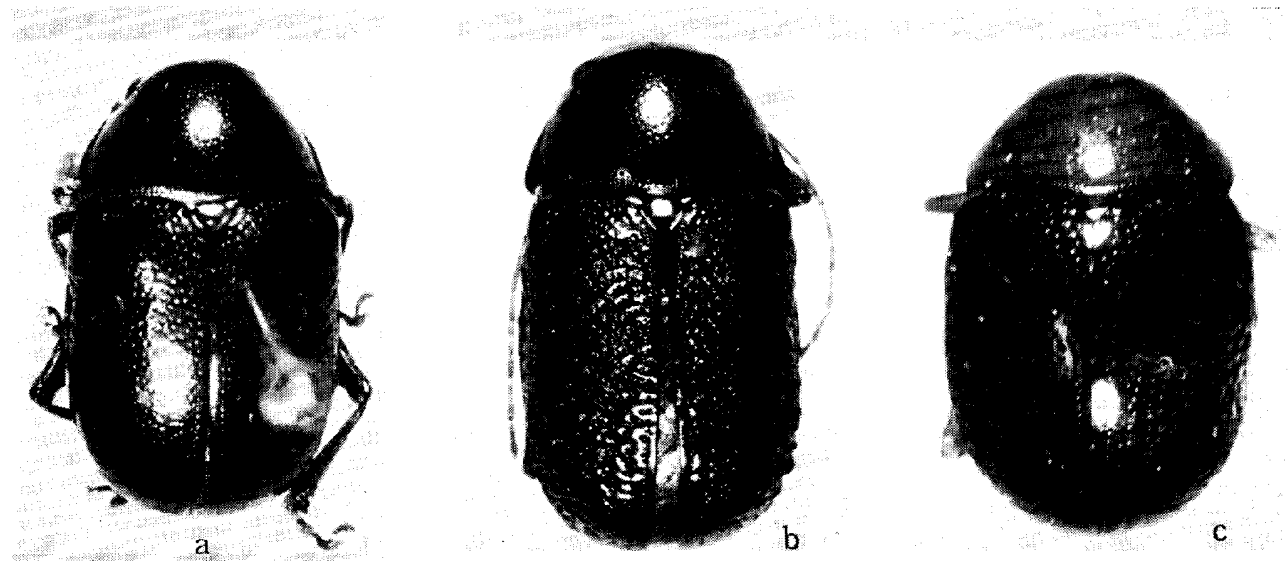

Fig. 3. a, Chrysolampra Eaosensis Kimoto \& Gressitt, n. sp.; h, C. mouhoti Baly ; c, C. minuta Kimoto \& Gressitt, n. sp.

punctures smooth and sparsely impressed by minute punctures; vertex with a shallow longitudinal furrow at middle. Antenna slender, filiform, nearly $3 / 4$ as long as body length; first segment robust, somewhat club-shaped ; second nearly half as long as first, robust; third nearly $11 / 2$ times as long as seocnd, slender ; fourth sligthly longer than third; fifth nearly $11 / 4$ times as long as fourth; sixth as long as fifth but apical part much wider; seventh subequal to sixth in length and shape; eighth nearly $3 / 4$ as long as seventh but much robuster ; ninth and tenth subequal to eighth in length and shape; eleventh slightly longer than tenth and its apex pointed. Pronotum transverse, about $21 / 5$ times as wide as long, side rounded, widest slightly behind middle and strongly narrowed anteriorly and less strognly so posteriorly; anterior and posterior corners each with a setigerous puncture; anterior margin concave, nearly straight at middle and posterior margin widely rounded, slightly produced posteriorly at middle; dorsal surface distinctly but not closely punctate and their interstices sparsely impressed by fine punctures; proepimeron slightly wrinkled, covered by fine hairs and impressed by fine punctures. Scutellum subpentagonal, with apex rounded; surface smooth, shining, nearly impunctate. Elytron slightly rounded at side, subbasal area not distinctly raised, without distinct subbasal transverse furrow; surface closely and distinctly punctate, and their interstices sparsely impressed by fine punctures.

Length $3.2-3.4 \mathrm{~mm}$.

Holotype (Type No. 2345, Kyushu Univ.), THAILAND: Chiang Mai Prov., Doi Suthep, $1000 \mathrm{~m}, 12$. vi. 1965, K. Morimoto. Paratopotypes: 5 exs., same data as the holotype (KU, BISHOP, KIмото). Paratypes : THAILAND: 2 exs., same data as the holotype but 19. vi. 1965; 4 exs., Doi Suthep, $1000 \mathrm{~m}, 4$ exs., 18. vi. 1965, Y. Miyatake; Tan Keo, Doi Suthep, 800 m, 4 exs., 10. vi. 1965, Y. 
Miyatake (KU) ; Doi Suthep, 1000 m, 1 ex., 12. vi. 1965, P. D. Ashlock; Chiangmai Prov., Chiang Dao, 2 exs., 15. vi. 1965, P. D. Ashlock (BISHOP).

This new species somewhat resembles C. cuprithorax Chen, in having the similar coloration of the dorsal surfaces, but differs in being the body length shorter, and having the ventral surfaces not entirely obscure blue.

Chrysolampra mouhoti Baly (Fig. 3b)

Chrysolampra mouhoti Baly, 1864, J. Entomol. 2: 220 (Siam ; BM).

Chrysolampra marginicollis Berlioz, 1917, Ann. Soc. Entomol. Fr., 86: 307 (Haut-Laos: Vientiane). New synonymy.

Distribution : Thailand, Laos, Vietnam.

We could not trace the type location of Chrysolampramarginicollis Berlioz. However, judging from the original description made by Berlioz, his species is no doubt identical with mouhoti Baly, from Siam.

MATERIAL EXAMined. THAILAND : Kanchanaburi, 8 exs., 21. v. 1962 (BANGKHEN). LAOS: Sayaboury Prov., Sayaboury, 6 exs., 5. v. 1965, 1 ex., 17. v. 1965, 2 exs., 30. v. 1965, Native collr ; Vientiane, 2 exs., 3. v.-3. vi. 1960, S. Quate \& L. W. Quate (BISHOP) ; Umgeb. Vientiane, 7 exs., iii-vi. 1963; Umgeb. Pakse, 1 ex., 1963; Umgeb. Vanky, 1 ex., 1964 (MUNCHEN). VIETNAM : Riv. de Chepone, Annam, 1 ex., 9. iv. 1927, Mrs. Dora E. Wright (CAS).

Chrysolampra rufimembris Pic

Chrysolampra rufimembris Pic, 1926, Mel. Exot. Entomol. 45: 13 (Tonkin; PARIS).

Distribution : Vietnam.

No additional material.

Chrysolampra splendens Baly (Fig. lb)

Chrysolampra splendens Baly, 1859, Ann. Mag. Nat. Hist. ser. 3, 4: 126 (China ; BM). -Berlioz, 1917, Ann. Soc. Entomol. Fr. 86: 307 (Ham-Laos : Keng-Trap). --Weise, 1922, Tijdschr. Entomol. 65: 46 (Fukien). - Chen, 1935, Sinensia 6(3): 244, fig. 5 (China). -Gressitt \& Kimoto, 1961, Pac. Insects Monogr. 1A: 285 (China).

Chry'solampraverrucosa Lef èvre, 1890, Nouv. Arch. Mus. Paris, ser. 3, 2: 192 (Laos: LuangPrabang; PARIS) ; 1893, Ann. Soc. Entomol. Fr. 1893: 115 (Tonkin: Lang-Son) ; 1904, Miss. Pavie Indo-China, ser. 2, 3 (Zool.) : 149. --Chen, 1935, Sinensia 6(3): 264 (Tonkin, Laos). -Gressitt \& Kimoto, 1961, Pac. Insects Monogr. 1A: 285 (Indo-China). New synonymy.

Chrysolampra festiva Lef èvre, 1893, Ann. Soc. Entomol. Fr. 1893 : 116 (Cochinchina) .--Berlioz, 1917, Ann. Soc. Entomol. Fr. 86: 307 (Haut-Laos: Vientiane). --Chen, 1935, Sinensia 6 (3) : 246 (S. China, Cochinchina). -Gressitt \& Kimoto, 1961, Pac. Insects Monogr. 1A: 284 (Cochinchina, S. China). New synonymy.

Chrysolampra testaceicornis Pic, 1907, Échange 23:135 (Tonkin; PARIS). N ew synonymy.

Chrysolampra coerulea Pic, 1926, Mel. Exot. Entomol. $45: 12$ (Tonkin; PARIS). New synonymy.

Chrysolampra tuberculata Pic, 1926, Mél. Exot. Entomol. 45: 13 (Tonkin; PARIS). New synonymy. 
Distribution : Cambodia, Laos, Vietnam, S. China.

We could not trace the type location of C. verrucosa Lefevre and C. festiva Lefevre. According to the original description of verrucosa Lefevre, the body length is described as $15-17 \mathrm{~mm}$. Chen (1935) cited the body length as $9.5 \mathrm{~mm}$ and separated this species from splendens Baly, in having the antenna enitrely brownish. However, this seems to be an infraspecific variation.

Material examined. LAOS: Vientiane Prov., Ban Van Eue, 4 exs., 15. v. 1965, 2 exs., 15-31. v. 1965, 1 ex., 31. v. 1966, Native collr ; Ban Van Eue, 20 km E of Phou-kow-kuei, 2 exs., 1-15. v. 1965, J. A. Rondon; Phou Kou Khouei, 1 ex, 31. v. 1966, Native collr; Sayaboury Prov., Sayaboury, 1 ex., 17. v. 1966, 2 exs., 20. v. 1966, Native collr (BISHOP) ; Umgeb. Vientiane, 7 exs., iii-vi. 1963, Umgeb. Pakse, 2 exs., 1963; Umgeb. Paklay, 1 ex., 1963 (MUNCHEN). VIETNAM: Hoa-Binh, Tonkin, 18 exs., A. Cooman; Monties Mauson, Tonkin, $2-300 \mathrm{~m}, 2$ exs., iv.-v. H. Fruhstorfer (FREY).

\section{Genus Iphimoides Jacoby}

Iphimoides Jac., 1883, Notes Leyden Mus. 5: 200 (type: Iphimoides celebensis Jacoby, Is. Saleyer). Lefevre, 1885, Mém. Soc. Sci. Liege, ser. 2, 11: 22. Gressitt \& Kimoto, 1961, Pac. Insects Monogr. 1A: 286.

\section{KeY TO SPECIES OF Iphimoides}

Reddish brown, elytron yellowish brown with suture and interior margin of epipleura blackish; dorsal surfaces impressed with distinct punctures, and their interstices smooth and shining; antenna pitchy black with four basal segments reddish brown; legs pitchy brown with femora reddish brown; length 6.0-6.3 mm

suturalis

Reddish brown, elytron bluish black; dorsal surfaces closely impressed with distinct punctures and their interstices finely granulate; antenna reddish brown with three apical segments blackish especially apical portion ; legs reddish brown with basal portion of posterior tibia slightly infuscate; length 7.0-8.6 $\mathrm{mm}$.

binhanus

Iphimoides binhanus Pic (Fig. 4b)

Iphimoides binhanus Pic, 1928, Bull. Soc.Zool.Fr. 53: 378 (Tonkin; PARIS).

Distribution : Laos, Vietnam.

Material examined. LAOS : Umg. Vientiane, 1 ex., iii-vi. 1963, Umgeb. Pakse, 1 ex., 1963 (MUNCHEN). VIETNAM: Hoa-Binh, Tonkin, 2 exs., A. Cooman (FREY).

Iphimoides suturalis Pic (Fig. 4c)

Iphimoides suturalis Pic, 1928, Bu1!. Soc. 2001. Fr. 53 : 378 (Tonkin; PARIS). -Gressitt \& Kimoto, 1961, Pac. Insects Monogr. 1A: 286 (Tonkin, S. China).

Distribution: vietnam, S. China. 


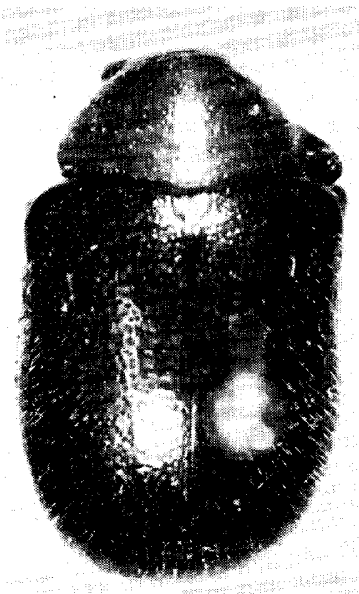

a

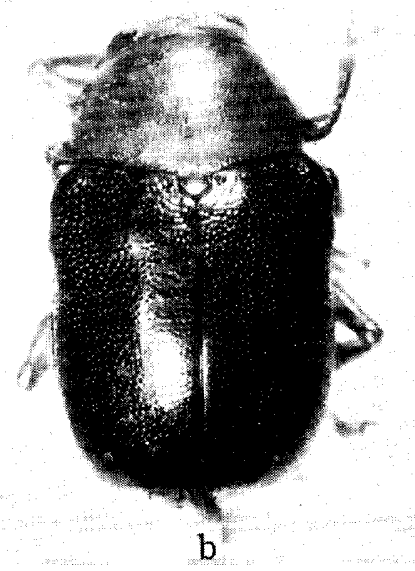

b

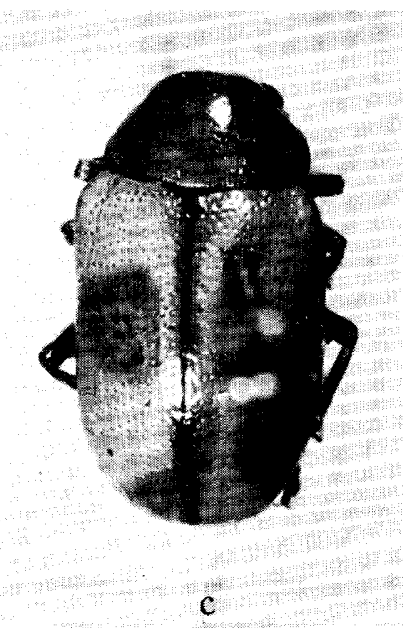

c.

Fig. 4. a, Paracrothinium collare Kimoto \& Gressitt, n. sp. b, Iphimoides binhanus Pic; c, I. suturalis $\mathrm{Pic}$.

Material examined LAOS: Sayaboury Prov., Sayaboury, 1 ex., 21. vi. 1966, Native collr (BISHOP).

\section{Genus Clisitherella Chen}

Clisitherella Chen, 1940, Sinensia 11: 207 (type : Clisitherella suturalis Chen, Tonkin).

\section{Clisitherella suturalis Chen}

Clisitherella suturalis Chen, 1940, Sinensia 11: 208 (Tonkin).

Distribution: Vietnam.

Body oblong subovate, moderately convex ; rufous or rufo-piteous; elytron yellow-brown, sutural and lateral margins reddish piceous; antenna red brown with five apcial segments deep fusco-rufous ; breast and abdomen ferrugineous ; length $5 \mathrm{~mm}$.

We could not check the type of this species.

\section{Genus Parascela Baly}

Parascela Baly, 1878, J. Linn. Soc. Lond., 14 : 252 (type : Pseudocolaspis cribrata Schaufuss, from Hongkong).-Lefèvre, 1885, Mém. Soc. Sci. Liege, ser. 2, 11: 64.-Chen, 1933, Sinensia 6(3): 297; 1940, ibid. 11(5-6): 489. --Gressitt \& Kimto, 1961, Pac. Insects Monogr. 1A: 237.

Pseudoparascela Pic, 1935, Mel. Exot. Entomol. 65: 1 (type: Pseudoparascela jeanvoinei Pic, from Tonkin). New synonymy.

Parascela cribrata (Schaufuss) (Fig. la)

Pseudocolaspis cribrata Sch., 1871, Nunguam Otiosus 1: 200 (Hong Kong). 
Parascelacribrata: Baly, 1878, J. Linn. Soc. Lond. 14: 252. -Gressitt\& Kimoto, 1961, Pac. Insects Monogr. 1A: 237 (China). Kimoto \& Gressitt, 1966, Pac. Insects 8(2): 507, fig. 11a (Ryukyu Is.).

Pseudoparascela jeanvoinei Pic, 1935, Mel. Exot. Entomol. 65: 1 (Tonkin; PARIS). New synonymy.

Basilepta hirta Chen, 1935, Sinensia 6(6): 769, fig. 1 (Yunnan). -Gressitt \& Kimoto, 1961, Pac. Insects Monogr. 1A:237 (=cribrata).

Parascela hirta: Chen, 1940, Sinensia 11(5-6): 485, 503 (China).

Distribution: Vietnam, S. China, Ryukyu Is.

No additional material.

\section{Genus Callisina Baly}

Callisina Baly, 1860, J. Entomol. 2: 30 (type : Callisina fasciata Baly, from Mallaca, Borneo). Chapuis, 1867, Genera Coleopt. 10: 246. -Lefèvre, 1885, Mém. Soc. Sci. Liege, ser. 2, 11: 63. Jacoby, 1908, Fauna India, Coleopt. 2 : 362. -Chen, 1935, Sinensia 6(3): 326 ; 1940, ibid.11(5-5): 488. Gressitt \& Kimoto, 1961, Pac. Insects Monogr. 1A: 214.

\section{Key to spectes of Callisina}

1 (0). Elytron without three pairs of tubercles laterally; elytron blackish with two or three pairs of markings brownish

Elytron with three pairs of tubercles laterosubbasally; elytron reddish brown with sutural and lateral margins together with a subtriangular laterosubbasal marking pitchy black; reddish brown, antenna black with basal segments brownish; length $5.0 \mathrm{~mm}$

trigibbosa

2 ( 1 Elytron pitchy black with basal, postmedian and apical markings reddish brown; pronotum reddish brown with basal portion pitchy black; ventral surfaces reddish brown; antenna black with basal segments brownish; legs reddish brown; length $5.8 \mathrm{~mm}$

Elytron black with basal and postmedian markings reddish brown; head black with a reddish marking at middle in most specimens; pronotum pitchy black; ventral surfaces black to pitchy brown; antenna black with basal segments brownish; legs black to pitchy brown ; length 4.8 $6.8 \mathrm{~mm}$

quadripustulata

\section{Callisina fasciata Baly (Fig. 5a)}

Callisina fasciata Baly, 1860, J. Entomol. 1 : 30, pl. 1, fig. 6 (Malacca, Borneo; BM) ; 1867 , Trans. Entomol. Soc. Lond., ser. 3, 4(2): 153 (Malacca, Borneo).

Distribution : Thailand, Malaya, Borneo.

Material examined. Thailand: Kao Chong, nr. Trung, 1 ex., 26. vi. 1965, K. Morimoto (KU).

Callisina quadripustulata Baly (Fig. 5b)

Callisina quadripustulata Baly, 1864, Descr. n. gen. \&spec. Phytoph., 11 (Java; BM) ; 1867, Trans. Entomol. Soc. Lond., ser. 3, 4(2): 154 (Java). Lefèvre, 1893, Ann. Soc. Entomol. Fr., $1893: 121$ (Cochinchina).

Callisina mouhoti Baly, 1864, Descr. n. gen. \&spec. Phytoph., 11 (Cambodia; BM) ; 1881, 
Trans. Entomol. Soc. Lond., 1881: 503 (Siam). Lefèvre, 1893, Ann. Soc. Entomol. Fr. 1893 : 121 (Cochinchina). New synonymy.

Callisina Zaosensis Pic, 1928, Mél. Exot. Entomol. 52: 28 (Laos ; PARIS). New synonymy.

Distribution : Thailand, Laos, Cambodia, Vietnam, Java.

Material examined. THAiland: Doi Suthep, $1000 \mathrm{~m}$, Chiang Mai Prov., 1 ex., 12. vi. 1965, 1 ex., 18. vi. 1965, Y. Miyatake; Khao Yai Nat. Park, 3 exs., 5. vi. 1965, K. Morimoto, 1 ex., 6. vi. 1965, Y. Miyatake (KU). CAMBODIA: Damrey Phong, 1 ex., 14-16. iv. 1961, N. R. Spencer (BISHOP). LAOS: Sayaboury Prov., Sayaboury, 1 ex., 5. v. 1966, Native collr; Wapikhamthong Prov., Khong Sedone, 1 ex., 16. v. 1965, Native collr (BISHOP); Umgeb. Vientiane, 1 ex., iii-vi. 1963 (MUNCHEN). VIETNAM: Kontum, $\mathrm{N}$ of Pleiku, $550 \mathrm{~m}, 1$ ex., 13. v. 1960, L. W. Quate (BISHOP).

Callisina trigibbosa $\mathrm{Pic}$

Callisina trigibbosa Pic, 1928, Mél.Exot. Entomol. 52: 28 (Indo-China: Chapa;PARIS).

Distribution : Vietnam.

No additional material.

\section{Genus Pagria Lefèvre}

Colposcelis Dejean, 1835, Cat. Coleopt. ed. 2: 408 (nec Dejean, 1834, ibid. ed. 2: 185, Tenebrionidae, Col.) ; 1837, ibid. ed. 3: 432. --Chevrolat, 1849, in d'Orbigny, Dict. Univ. d'Hist. Nat., 13 (type: Colaspis viridiaenea Gyllenha1). --Monrós \& Bechyné, 1956, Entomol. Arb. Mus. Frey 7(3): 1126. Gressitt \& Kimoto, 1961, Pac. Insects Monogr. 1A: 232. Kimoto, 1964, J. Fac. Agr. Kyushu Univ.13(2): 242. -Selman, 1965, Bull. Brit. Mus. (Nat. Hist.), Entomol. 16(3): 147.

Pagria Lef., 1884, Bull. Soc. Entomol. Fr. ser. 6, 4: 67 (P. suturalis Lef.; Africa; first species listed) ; 1885, Mém. Soc. Sci. Lièg, ser. 2, 11: 62. -Jacoby, 1908, Fauan India, Coleopt. 2: 356 (type designated as suturalis). -..Chen, 1935, Sinensia 6 (3) : 324; 1940, ibid. 11(5-6): 488. Chûjô, 1956, Philip. J. Sci. 85(1): 56. -Aslam, 1968, J. Nat Hist. 2: 128.

Aphthonesthis Weise, 1895, D. Entomol. Z. 1895: 329 (type : Aphthonesthis concinna Weise; W. Africa).

\section{Key to SPECIES of Pagria}

Pronotum nearly $13 / 4$ times as wide as long, head with vertex impressed with large punctures and their interstices much narrower than their diameters;

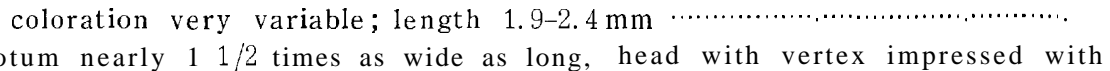

Pronotum nearly $11 / 2$ times as wide as long, head with vertex impressed with general color reddish brown, with top of vertex, a pair of median markings of pronotum, and basal and postmedian markings of elytron black; length $2.5-2.7 \mathrm{~mm}$ 

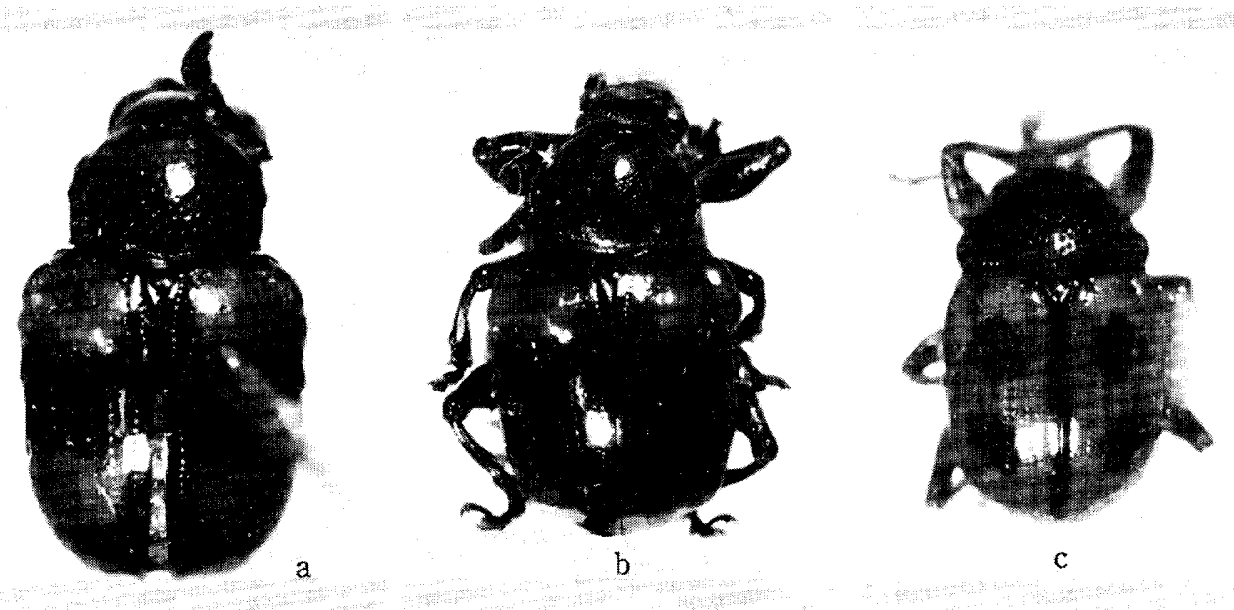

Fig. 5. a, Callisina fasciata Baly ; b, G. quadripustulata Baly ; c, Pagria sexmaculata Kimoto \& Gressitt, n. sp.

\section{Pagria sexmaculata Kimoto \& Gressitt, n. sp. (Fig. 5c)}

Body oval; general color reddish brown, head with top of vertex pitchy black; pronotum with a pair of median markings and entire margins pitchy black; scutellum entirely pitchy black; elytron with basal, postmedian and lateromedian markings together with sutural margin pitchy black; antenna mostly dark reddish brown; ventral surface of prothorax mostly pitchy black ; legs with apical and basal portions of femora pitchy black.

Head with wide sulcus above eye, surface somewhat rugose, strongly and very closely punctate, and interstices of punctures much narrower than their diameters; vertex with a short shallow longitudinal furrow at middle. Antenna slender, nearly $2 / 3$ as long as body length; first semgent robust, somewhat club-shaped; second nearly $3 / 5$ as long as first, robust ; third as long as second, but much slenderer; fourth nearly $11 / 3$ times as long as third, slender ; fifth nearly $3 / 4$ as long as fourth ; sixth subequal to fifth in length and shape; seventh nearly $11 / 4$ times as long as sixth; eighth and ninth subequal to seventh in length and shape; tenth slightly shorter than ninth; eleventh nealry $11 / 4$ times as long as tenth and its apex pointed. Pronotum transverse, nearly $11 / 2$ times as wide as long; side rounded, widest slightly behind middle and strongly narrowed anteriorly and less strongly so posteriorly; anterior and posterior corners somewhat thickened and each with a setigerous puncture; anterior margin slightly rounded anteriorly and posterior margin rounded posteriorly and slightly produced at middle; anterior sulcus distinct on lateral portion and shallower at middle and impressed by a row of distinct punctures; dorsal surface closely and distinctly punctate and their interstices smooth, shining, and convex especially at side; proepimeron closely and dis- 
tinctly punctate on basal portion; metasternum sparsely impressed by small punctures. Scutellum subtrigonate, with apex rounded; surface convex, smooth, shining, impunctate. Elytron rounded at side, widest sligthly behind humerus and gradually narrowed posteriorly and apex rounded; subbasal area slightly convex, subbasal transverse furrow obsolete ; punctures regularly arranged in longitudinal rows, and punctures larger and deeper on basal area and much finer and shallower on apical area, and their interstices sparsely impressed with minute punctures; humerus distinctly raised, and with a short costa starting from humerus. Each femur with a minute tooth beneath.

Length $2.5-2.7 \mathrm{~mm}$.

Holotype (BISHOP), THAILAND: Chiangmai Prov., Doi Suthep, 1-5. iv. 1958, T. C. Maa. Paratopotypes: 2 exs., same data as the holotype (BISHOP, KIMOTO).

This new species somewhat resembles Pagria signata (Motschulsky), but differs in having the head more closely and somewhat rugosely punctate.

\section{Pagria signata (Motschulsky)}

Metachroma signata Motsch., 1858, Etud. Entomol. 7: 110 (Burma).

Nodostoma consimile Baly, 1874, Trans. Entomol. Soc. Lond., 1874 : 168 (Japan ; BM). -Jacoby, 1908. Fauna India, Coleopt. 2:356 (=signata).

Nodostoma flavopustulatum Baly, 1874, Trans. Entomol. Soc. Lond., 1874: 169 (Japan ;BM). Chûjô \& Kimoto, 1961, Pac. Insects 3(1): 143 (=signata).

Pagria signata: Jacoby, 1908. Fauna India, Coleopt. 2: 356, fig. 125 (India, Burma, Ceylon, China, Japan). - Chen, 1935, Sinensia 6(3): 325 (Siberia, China, Burma, S. India, Ceylon, Japan). -Chûjô, 1956, Philip. J. Sci. 85(1): 58 (Formosa). --Gressitt, 1955, Insects Micronesia 17(1): 12 (Guam).

Pagria flavopustulata: Jacoby, 1908, Fauna Idnia, Coleopt, 2: 356 (India, Japan, Sumatra). -Chûjô, 1956, Philip. J. Sci. 85(1): 62 (Formosa).

Pagria flavopustuiata ab. bicolor Weise, 1922, Philip. J. Sci. 21 : 451 (Philippines).

Pagria ingibbosa Pic, 1929, Mél. Exot. Entomol. 53: 35 (Tonkin; PARIS). New synonymy.

Pagria signata var. anceyi Pic, 1929, Mel. Esot. Entomol. 53: 35 (Japan).

Pagria signata varr. rufithorax, innotata Pic, 1929, Mel. Exot. Entomol. 53: 35 (Tonkin).

Pagria signata ab. ussuriensis Roubal, 1929, Boll. Soc. Entomol. Ital., 61 (5-6) : 98 (Vladivostock).

Pagria signata grandis Chûjô, 1938, Arb. Morph. Taxon. Entomol. Berlin-Dahlem 1(5): 28 (Formosa).

Pagria annulicornis Pic, 1950, Échange 66: 4 (Indo-China; PARIS). New synonymy.

Pagria diversepunctata Pic, 1950, Diversites Entomol. 7: 3 (Tonkin; PARIS).

Colposcelis signata: Gressitt \& Kimoto, 1961, Pac. Insects Mongor. 1A:232(China,Hainan).Chûjô, 1961, Nature and Life in SE Asia, Osaka 1: 349 (Thailand: DoiDoa).-Kimoto, 1964, J. Fac. Agr. Kyushu Univ. 13(2): 242 (Japan, Ryukyu Is.).

Distribution : India, Burma, Thailand, Laos, Vietnam, China, Siberia, Korea, Taiwan, Philippines, Indonesia, Japan, Ryukyus, Bonin Is., Guam.

Body short oval, convex, with dorsal surfaces glabrous; coloration variable; pronotum (a) yellowish or reddish brown, (b) entirely black; elytron (a) reddish or yellowish brown with subbasal area paler, sutural margin more or 
less infuscate in many cases, lateral marign often entirely or partly infuscate, postbasal depression and posthumeral area frequently infuscate, (b) entirely piceous to black; antenna and legs yellowish brown, the former more or Iess infuscate at apical segments ; length 1.8-2.4 mm.

Material EXAmined. THAilAnd: Maesa Water Fall, Chiang Mai Prov., 12 exs., 16. vi. 1965, K. Morimoto; Doi Suthep, 1000 m, Chiang Mai Prov., 3 exs., 10. vi. 1965, K. Morimoto; Tan Keo, Doi Suthep, 800 m, Chiangmai Prov., 1 ex., 10. vi. 1965, Y. Miyatake; Chiangmai, 1 ex., 9. vi. 1965, K. Morimoto, 1 ex., 17. vii. 1973, sweeping of paddy field, K. Yano; Chieng Dao, 13 exs., 15-16. vi. 1965, K. Morimoto \& Y. Miyatake; Ban Tap Dua, Chieng Dao, 15. viii. 1973, sweeping of paddy field, K. Yano; Fang, 4 exs., 14. vi. 1965, K. Morimoto \& Y. Miyatake ; Khao Yai Nat. Park, 10 exs., 6. vi. 1965, K. Morimoto \& Y. Miyatake ; Khao Chong, nr. Trung, 5 exs., 24-27. vi. K. Morimoto; Kor Hong, 1 ex., 23. vi. 1965, K. Morimoto; Pew, Chanta Buri, 2 exs., 29. viii. 1973, S. Nakao (KU) ; Chiamgmai Prov., Chiang Dao, 5 exs., 15. vi. 1965, P. D. Ashlock; Chiangmai Prov., Doi Suthep, 900 m, 1 ex., 14. xi. 1957, J. L. Gressitt; Chiangmai Prov., Fang (Agr. Exp. Station), 600 m, 1 ex., 14. vi. 1965, P. D. Ashlock; Chiangmai Prov., Doi Pui, 1 ex., 2. iv. 1958, T. C. Maa; Chanthaburi Prov., Prew, 2 exs., 25-30. iv. 1958, T. C. Maa; Pakchong, 100 m, NE of Bankok, 2 exs., 2-3. xii. 1957, J. L. Gressitt; Nakhon Nayok Prov., Khao Yai Nat. Park, 1 ex., 5. vi. 1965, P. D. Ashlock (BISHOP). LAOS: Ban Van Heue, $20 \mathrm{~km}$ E of Phou-kow-kuei, 2 exs., 1-15. v. 1965, J. A. Rondon; Dong Dok, 1 ex., 26. xi. 1965, Native collr (BISHOP). VIETNAM: Hoa-Binh, Tonkin, 5 exs., A. Cooman, 2 exs., J. Clermont (FREY) ; Dalat, $1500 \mathrm{~m}, 3$ exs., 29. iv.-4. v. 1960, L. W. Quate; 18 km NW of Dalat, $1300 \mathrm{~m}, 1$ ex., 4-5. v. 1960, L. W. Quate; $20 \mathrm{~km} \mathrm{~S}$ of Dalat, 1300 m, 1 ex., 12. ix. 1960, J. L. Gressitt ; Fyan, 1200 m, 4 exs., 11. vii.-9. viii. 1961, N. R. Spencer; Blao (Balao), 500 m, 1 ex., 14-21. x. 1960, C. M. Yoshimoto ; 7 km SE of Dilinh (Djiring), 990 m, 1 ex., 2. v. 1960, R. E. Leech(BISHOP)

\section{Genus Nodina Motschulsky}

Nodina Mots., 1853, Etud. Entomol. 7: 108 (first species listed: Nodina pusilla Motschulsky from India). -Chapuis, 1874, Genera Coleopt. 10: 262. -Lefèvre, 1885, Mém. Soc. Sci. Liege, ser. 2, 11: 61. Jacoby, 1908, Fauna India, Coleopt. 2: 292 (type designated as N. pusilla Mots.). -Chen, 1935, Sinensia 6 (3) : 297: 1940, ibid. 11 (5-6) : 488. -Chûjô, 1956, Philip. J. Sci. 85(1) : 10. -Gressitt \& Kimoto, 1961, Pac. Insects Monogr. 1A:205. -Kimoto, 1964, J. Fac. Agr. Kyushu Univ. 13(2): 243.

\section{Key to SPECIES OF Nodina}

$1(0)$. Anerior margin of clypeus angularly notched or rather strongly produced on either side of apex and forming semicircular emargination …........ 2 Anterior margin of clypeus widely rounded or nearly straight $\ldots \ldots \ldots \ldots \ldots \ldots \ldots . . . . . .6$

$2(1,$. Proepimeron with surface impunctate, partly or entirely granulate $\cdots \ldots \ldots \ldots \ldots \ldots 3$ Proepimeron with surface sparsely impressed by distinct punctures, and 
partly granulate on lateral and basal portion ; anterior margin of clypeus angularly notched; dorsum greenish aeneous, beneath black; legs and labrum reddish brown; antenna reddish brown with apical seg-

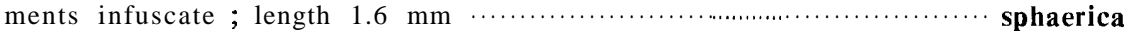

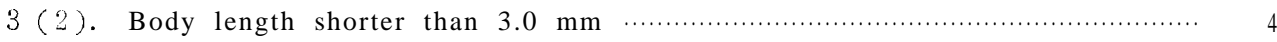

Body length longer; anterior margin of clypeus angularly notched ; general color bluish black, labrum reddish brown; antenna reddish brown with apical segments infuscate, legs black with tarsi and apical part of tibiae reddish brown; length $3.5-3.7 \mathrm{~mm} \ldots \ldots \ldots \ldots \ldots \ldots \ldots \ldots \ldots \ldots \ldots \ldots$ major

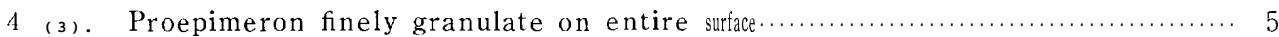

Proepimeron granulate on basal part only; greenish aeneous, beneath black; legs and labrum reddish brown; antenna yellowish brown with apical segments somewhat infuscate ; length 1. 6-2. $2 \mathrm{~mm} \ldots \ldots \ldots \ldots \ldots \ldots \ldots$ similis

5 (4). Anterior margin of clypeus somewhat angularly notched; legs entirely reddish brown; dorsum generally greenish aeneous and beneath black, in some specimen dorsum bluish ; labrum reddish brown; antenna reddish brown with apical segments infuscate ; length $1.4-2.3 \mathrm{~mm}$........ pusilla

Anterior margin of clypeus strongly produced on either side of apex and forming semicircular emargination; legs blackish with tarsi and apical part of tibiae brownish in various degrees, and in most pale colored specimen tibiae entirely brownish; dorsum bluish black; labrum pitchy black to reddish brown; length $1.4-2.9 \mathrm{~mm}$

coerulea

6 (1). Anterior part of clypeus finely punctate and their interstices finely granulate and its anterior margin nearly straight; proepimeron with surface sparsely impressed by distinct punctures and partly granulate

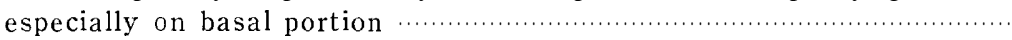

Anterior part of clypeus distinctly punctate and their interstices smooth, and its anterior margin widely rounded....

7 (6). Head finely granulate almost entire surface; elytron without sharp ridge laterally in both sexes; dorsum greenish cupreous or bluish black; legs blackish with basal part of femora and apical part of tibiae and entire tarsi brownish; labrum pitchy black; antenna blackish with basal segments brownish; length 2.1-2.5 mm

Head somewhat granulate almost near anterior margin only, and interstices of punctures impressed by minute punctures; elytron with a sharp lateral ridge in female; dorsum greenish cupreous or bluish black; legs reddish brown, but in some specimen blackish in various degrees; labrum reddish to pitchy brown; antenna reddish to pitchy brown with apical segments much darker; length 1.9-Z. $6 \mathrm{~mm} \ldots$ punctostriolata

8 (6). In female elytron with two lateral costae, of which inner one much longer than outer one; dorsum metallic greenish aeneous; labrum and legs reddish brown, and antenna deep yellowish brown with apical

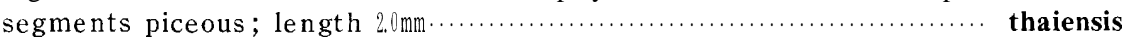

In female elytron with three lateral costae, of which middle one commencing on humeral callus and interrupted at middle; dorsum greenish cupreous, labrum and legs entirely reddish brown; antenna reddish brown with apical segments infuscate ; length 1.6-1. $9 \mathrm{~mm} \cdots \cdot$ minutissima 
Nodina clypeata Kimoto \& Gressitt, n. sp. (Fig. 6a)

Ovate ; dorsum greenish cupreous or bluish black; legs blackish with basal portion of femora and apical part of tibiae and entire tarsi brownish; labrum pitchy black; antenna blackish with basal segments brownish.

Head with surface fienly granulate, granulation somewhat obsolate on top of vertex, and distinctly and fairly closely punctate; vertex with a short shallow longitudinal furrow at middle; anterior margin of clypeus widely rounded. Antenna robust, short, nearly half as long as body length; first segment robust, somewaht club-shaped, nearly twice as long as wide; second subequal to first in length but more slenderer; third and fourth fairly slender, and subequal in length and shape to each other but shorter than second; fifth to tenth strongly swollen, each about as broad as long, and somewhat flattened. Pronotum nearly $13 / 4$ times as long as wide, disc evenly convex, smooth, and strongly and closely puncatate and their interstices impressed by minute punctures. Scutellum smooth, shining, slightly broader than long, obtusely rounded behind, sparsely impressed by minute punctures. Elytron with side rounded, disc smooth, with distinct punctures in 11 rows, interspaces flat, smooth, shining, impressed by minute punctures. Proepimeron with surface sparsely impressed by distinct punctures and granulate on basal portion.

Length 2.1-2.5 $\mathrm{mm}$.

Holotype (Type No. 2346, Kyushu Univ.), THAILAND: Chiang Mai Prov., Chiang Dao, 2. xi. 1970, S. Nakao. Paratopotype : 1 ex., same data as the holotype (KIMOTO). Paratypes : THAILAND: Chiang Mai Prov., Fang (Agr. Exp. Station), 600 m, 1 ex., 14. vi. 1965, P. D. Ashlock (BISHOP) ; Fang Spa, 16 exs., 21. xi. 1968, K. Hatta (EHIME) ; Fang, 1 ex., 14. vi. 1965, Y. Miyatake (KU) ; Chiang Mai, 5 exs., 3. xii. 1962 (BANGKHEN). LAOS: Sedone Prov., Pakse, 2 exs., 15. v. 1965, P. D. Ashlock, 2 exs., 31. v. 1965, Native collr; Paksong, 2 exs., 17. v. 1965, 2 exs., 18. v. 1965, P. D. Ashlock (BISHOP).

This new species somewhat resembles N. sphaerica Chen, but differs in having clypeus and vertex finely granulate, and from N. punctostriolata, clypeus and vertex more finely granulate.

\section{Nodina coerulea Chen}

Nodina coerulea Chen, 1940, Sinensia 11: 494 (Tonkin:Thyen-Quan; PARIS).

Distribution : Thailand, Laos, Vietnam.

Material examined. THAiland : Chiang Mai Prov., Fang (Agr. Expt. Station), 600 m, 1 ex., 14. vi. 1965, P. D. Ashlock (BISHOP) ; Fang, 1 ex., 14. vi. 1965, Y. Miyatake; Doi Pui, 1300 m, 11 exs., 17-18. vi. 1965, K. Morimoto \& Y. Miyatake ; Doi Suthep, $100 \mathrm{~m}, 3$ exs., 18. vi. 1965, K. Morimoto; Mae Klang Water Fall, nr. Chom Thong, 1 ex., 11. vi. 1965, Y. Miyatake; Kao Chong, nr. Trung, 1 ex., 26. vi. 1965, K. Morimoto; Kao Chong Park, 1 ex., 1. iii. 1965, K. 


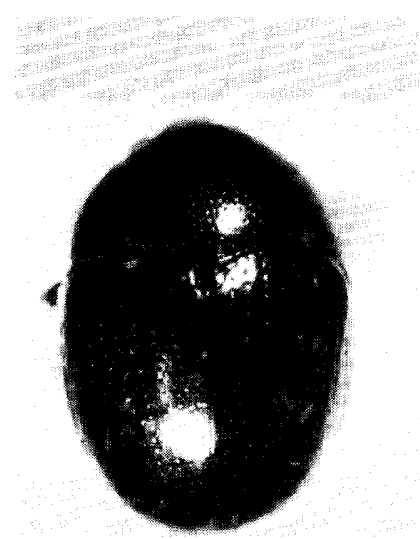

a

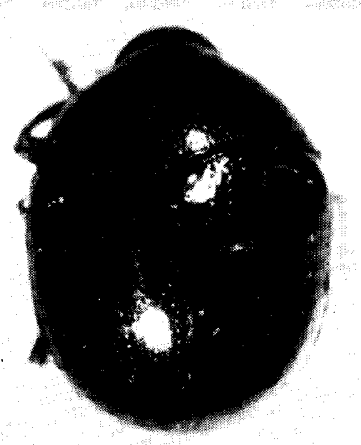

h

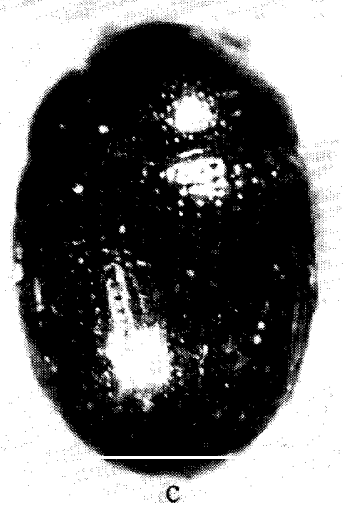

Fig. 6. a, Nodina clypeata Kimoto \& Gressitt, n. sp.: b, N. major Kimoto \& Gressitt. n. sp.; c, Nodia minutissima Kimoto \& Gressitt, n. sp.

Yasumatsu (KU). LAOS: Vientiane Prov., Vientiane, 1 ex., 22. vii. 1965, Native collr; Savanakhet Prov., Savanakhet, 1 ex., 15. iv. 1967, Native collr (BISHOP). VIETNAM: Fyan, 1200 m, 11 exs., 11. viii.-9. viii. 1961, N. R. Spencer ; Dalat, 1500 m, 1 ex., 26-27. ix. 1960, C. M. Yoshimoto, 3 exs., 28. iv.-4. v. 1960, L. W. Quate; Dalat, 1550 m, 2 exs., 11. ix. 1960, J. L. Gressitt; 20 km N of Pleiku, 650 m, 3 exs., 9. v. 1960, L. W. Quate ; Karyu Dannar, 200 m, 1 ex., 13-28. ii. 1961, N. R. Spencer; Ban Me Thuot, 570 m, 1 ex., 11. v. 1960, R. E. Leech; Ban Me Thuot, 500 m, 1 ex., 16-18. v. 1960, L. W. Quate, 1 ex., 20-24. xii. 1960, C. M. Yoshimoto; Di Linh (Djiring), $1200 \mathrm{~m}, 1$ ex., 22-28. iv. 1960, L. W. Quate; Ap. Hung-Lam, $21 \mathrm{~km}$ NW of Dilinh, 1100 m, 1 ex., 29. ix.-5. x. 1960, C. M. Yoshimoto ; Dai Lanh, N. of Nha Trang, 1. ex., 30. xi.-5. xii 1960, C. M. Yoshimoto; Blao (Balao), 500 m, 1 ex., 14-21. x. 1960, C. M. Yoshimoto (BISHOP).

\section{Nodina major Kimoto \& Gressitt, n. sp. (Fig. 6b)}

Spherical; general color bluish black, labrum reddish brown; antenna reddish brown with apical segments infuscate; legs black with tarsi and apical part of tibiae reddish brown.

Head with surface strongly and closely punctate, and their interstices closely impressed by minute pucntures, vertex with a short shallow longitudinal furrow at middle; anterior margin of clypeus angularly notched. Antenna robust, short, nearly half as long as body length; first segment robust, somewaht club-shaped, nearly twice as long as wide; second subequal to first in length but more slenderer; third and fourth fairly slender, and subequal in length and shape to each other but shorter than second; fifth to tenth 
strongly swollen, each about as broad as long, and somewhat flattened. Pronotum nearly twice as long as wide, disc evenly convex, smooth, and strongly and closely punctate and their interstices impressed by minute punctures. Scutellum smooth, shining, nearly impunctate, slightly broader than long, obtusely rounded behind. Elytron with side rounded, disc smooth, with distinct punctures in 11 rows, interspaces flat, smooth, shining, impressed by minute punctures. Proepimeron with surface entirely granulate, impunctate.

Length $3.5-3.7 \mathrm{~mm}$.

Holotype (Type No. 2347, Kyushu Univ.), THAILAND: Chiang Dao, 15. vi. 1965, K. Morimoto. Paratopotype : 1 ex., same deta as the holotype (BISHOP).

This new species somewaht resembles $N$. chinensis Weise, in being the size larger, but differs in having the legs blackish with tarsi and apical part of tibiae reddish brown.

\section{Nodina minutissima Kimoto \& Gressitt, n. sp. (Fig. 6c)}

Oblong oval ; dorsum greenish cupreous, labrum and legs entirely reddish brown; antenna reddish brown with apical segments infuscate.

Head with surface distinctly but not closely punctate, and their interstices smooth, shining ; vertex with a short shallow longitudinal furrow at middle; anterior margin of clypeus widely rounded. Antenna robust, short, nearly half as long as body length; first segment robust, somewhat club-shaped, nearly twice as long as wide; second subequal to first in length but more slenderer; third and fourth fairly slender, and subequal in length and shape to each other but shorter than second; fifth to tenth strongly swollen, each about as broad as long, and somewhat flattened. Pronotum nearly twice as long as wide, disc evenly convex, smooth, and strongly and closely punctate and their interstices impressed by minute punctures. Scutellum smooth, shining, slightly broader than long, obtusely rounded behind, nearly impuctate. Elytron with side rounded, disc smooth, with distinct punctures in 11 rows, interspaces smooth, shining, impressed by minute punctures; in female specimen elytron with three lateral costae, of which middle one commencing on humeral callus and interrupted at middle. Proepimeron with surface sparsely impressed by distinct punctures and granulate on basal portion.

Length 1.6-1. $9 \mathrm{~mm}$.

Holotype (BISHOP), VIETNAM: $10 \mathrm{~km}$ E of Ban Me Thuot, $570 \mathrm{~m}, 11 . \mathrm{v}$. 1960, R. E. Leech. Paratopotype: 1 ex., same data as the holotype (KIMOTO). Paratypes : VIETNAM: 1 ex., same data as the holotype, but 7. v. 1960; Dok Song, 76km SW of Ban Me Thuot. 1 ex., 19.-21. v. 1960, L. W. Quate; $17 \mathrm{~km} \mathrm{~S}$ of Dilinh, 1300 m, 1 ex., 6-13. x. 1960, C. M. Yoshimoto; Ap Hung-Lam, 21 km NW of Dilinh, 110 m, 1 ex., 29. ix.-5. x. 1960, C. M. Yoshimoto (BISHOP).

This new species somewhat resembles N. chalcosoma Baly, in having three lateral costae in female, but differs in being body length shorter, and having 
proepimeron sparsely impressed by distinct punctures, lateral ridges slightly lower and the anterior margin of clypeus widely rounded.

\section{Nodina punctostriolata (Fairmaire)}

Lamprosoma punctostriolatum Fairm., 1888, Ann. Soc. Entomol. Belg. 32: 37 (China: Kiangsi).

Lamprosoma minutulum Fairm., 1888, ibid. (China : Kiangsi). -Gressitt \& Kimoto, 1961, Pac. Insects Monogr. 1A : 212 (= punctostriolata).

Nodina chinensis Bryant, 1924, Ann. Mag. Nat. Hist. ser. 9, 14: 248 (China: Fukien; BM). Gressitt\& Kimoto, 1961, Pac. Insects Monogr, 1A:212 (=punctostriolata).

Nodina punctostriolata : Chen, 1932, Bull. Soc. Entomol. Fr. 1932: 108 (Eumolpinae) ; 1935, Sinensia 6(3): 301 (China). -Gressitt \& Kimoto, 1961, Pac. Insects Monogr. 1A: 212 (China, Hainan).

Nodina minutula: Chen, 1935, Sinensia 6(3): 301 (China).

Nodina fokienica Chen, 1935, Sinensia 6(3): 300 (new name for chinensis Bryant, nec Weise, 1922). Gressitt \& Kimoto, 1961, Pac. Insects Monogr. 1A:212 (=punctostriolata).

Nodina metallica Bryant, 1935, Ann. Mag. Nat. Hist. ser. 10, 20: 98 (new name for chinensis Bryant, nec Weise, 1922). Gressitt \& Kimoto, 1961, Pac. Insects Monogr. 1A: 212 $(=$ punctostriolata $)$.

Distribution : Thailand, Laos, Vietnam, Hainan, China.

Material examined. thailand: Chiangmai Prov., Chiangmai, 2 exs., 9. vi. 1965, P. D. Ashlock (BISHOP) ; Chiangmai, 1 ex., 3. xii. 1962 (BANGKHEN) ; Chiangmai, 1 ex., 12. vi. 1965, K. Morimoto ; Botanical Garden, Chiangmai, 1 ex., 12. vi. 1965, Y. Miyatake; Maesa Water Fall, 2 exs., 16. vi. 1956, K. Morimoto; Khao Yai Nat. Park, 8 exs., 5-6. vi. 1965, K. Morimoto \& Y. Miyatake; Muak Lek, $40 \mathrm{~km}$ SE of Sara Buri, 1 ex., 5. vi. 1965, Y. Miyatake (KU) ; Pak Chong, Korat Plateau, 1 ex., 14. iv. 1973, Y. Yoshiyasu (KIMOTO). LAOS: Dong Dok, 1 ex., 7. ix. 1965, Native collr; Muong Sing, NW of Luang Prabang, 650 m, 1 ex., 6-10. vi. 1960, S. Quate \& L. W. Quate; Borikhane Prov., Pakkading, 1 ex., 9. ix. 1965, Native collr; Vientiane Prov., Vientiane, 1 ex., 28. vii. 1965, Native collr (BISHOP). VIETNAM: Ban Me Thuot, $500 \mathrm{~m}, 1$ ex., 16-18. v. 1960, L. W. Quate ; $10 \mathrm{~km} \mathrm{E} \mathrm{of} \mathrm{Ban} \mathrm{Me} \mathrm{Thuot,} 570 \mathrm{~m}, 1$ ex., 11. v. 1960, R. E. Leech; M'Drak, E of Ban Me Thuot. 4-600 m, 2 exs., 8-19. xii. 1960, C. M. Yoshimoto ; Dak Song, $76 \mathrm{~km}$ SW of Ban Me Thuot, 3 exs., 19-21. v. 1960, S. Quate, L. W. Quate \& R. E. Leech; Dilinh (Djiring), 1200 m, 2 exs., 22-28. iv. 1960, L. W. Qaute ; 17 km S of Dilinh, 1 ex., 6-13. x. 1960, C. M. Yoshimoto; $30 \mathrm{~km} \mathrm{NW}$ of Dilinh, 1 ex., 27. iv. 1960, S. Quate \& L. W. Quate ; Ap Hung-Lam, $21 \mathrm{~km} \mathrm{NW}$ of Dilinh, $1100 \mathrm{~m}, 2$ exs., 29. ix.-5. x. 1960, C. M. Yoshimoto; $20 \mathrm{~km}$ $\mathrm{N}$ of Pleiku, $650 \mathrm{~m}, 2$ exs., 9. v. 1960, L. W. Quate ; Fyan, 900-1200 m, 2 exs., 11. vii. 9. viii. 1961, N. R. Spencer; Mt. Lang Bian, 1500-2000 m, 3 exs., 19. v.-8. vi. 1961, N. R. Spencer; Dalat, 1550 m, 3 exs., 11. ix. 1960, J. L. Gressitt; Dalat, 1500 m, 1 ex., 26-27. ix. 1960, C. M. Yoshimoto; 6 km S of Dalat, 1400-1500 m, 3 exs., 9. v.-7. vii. 1961, N. R. Spencer, 1 ex., 12. ix. 1960, J. L. Gressitt (BISHOP) ; Chapa, Tonkin, 3 exs. (FREY). 
Nodina pusilla Motschulsky

Nodina pusilla Motsch., 1858, Etud. Entomol. 7: 108 (India). -L.efèvre, 1889, Ann. Soc. Entomol. Fr. 1889: 290 (Annam: Qui-Nhon) ; 1893, ibid. 1893: 120 (Tonkin). -Jacoby, 1908, Fauna India, Coleopt. 2: 295 ("India").

Distribution : India, Thailand, Vietnam.

We could not check the type of this species. We follow Lefèvre's identification at present. Our material closely resembles Nodina parvula Jacoby from Burma, but differs in having the anterior margin of clypeus somewhat angularly notched. In parvula Jacoby, the anterior margin of clypeus is widely rounded.

Material examined. THAiland : Chiangmai Prov., Mae Klang, $340 \mathrm{~m}$, 1 ex., 11. vi. 1965, P. D. Ashlock (BISHOP) ; Maesa Water Fall, 1 ex., 16. vi. 1965, K. Morimoto ;Doi Suthep, 1000 m, 1 ex., 10. vi. 1965, K. Morimoto, 5 exs., 18. vi. 1965, K. Morimoto \& Y. Miyatake; Tan Keo, Doi Suthep, 800 m, 2 exs., 10. v. 1965, Y. Miyatake ;Doi Pui, 1685 m, 32 exs., 17. vi. 1965, Y. Miyatake ; Chiangmai, 1 ex., 9. vi. 1965, K. Morimoto; Mae Klang Water Fall, nr. Thorn Thong, 2 exs., 11. vi. 1965, Y. Miyatake ; Khao Yai Nat. Park, 10 exs., 5-6. vi. 1965, K. Morimoto \& Y. Miyatake; Kao Chong Park, 3 exs., 1. iii. 1965, K. Yasumatsu (KU) ; Fang, 1 ex., 22. xi. 1968, M. Sato ; Pak Chong, Korat Plateau, 1 ex., 14. iv. 1973, Y. Yoshiyasu; Saraburi, 14 exs., 28. i. 1968, K. Baba (KIMOTO). VIETNAM: Ban Me Thuot, 2 exs., 16-18. v. 1960, S. Quate \& L. W. Quate; $10 \mathrm{~km}$ E of Ban Me Thuot, 1 ex., 7. v. 1960, 2 exs., 11. v. 1960, R. E. Leech; Dak Song, $75 \mathrm{~km}$ SW of Ban Me Thuot, $870 \mathrm{~m}, 1$ ex., no date, R. E. Leech; Diling (Djiring), 900-1200 m, 3 exs., 22-28. iv. 1960, S. Quate \& L. W. Quate, 1 ex., 27. ix.-14. x. 1960, C. M. Yoshimoto; Ap Hung-Lam, 21 km NW of Dilinh, 1100 m, 3 exs., 29. ix.-5. x. 1960, C. M. Yoshimoto ; $20 \mathrm{~km} \mathrm{~N}$ of Pleiku, 1 ex., 650 m, 9. v. 1960, S. Quate ; Karyu Danar, 200 m, 1 ex., 13-28. ii. 1961, N. R. Spencer; Dalat, 1500 m, 1 ex., 26-27. ix. 1960, C. M. Yoshimoto (BISHOP).

\section{Nodina rotundata Motschulsky}

Nodina rotundata Motsch., 1858, Etud. Entomol. 7: 109 (India). Lefèvre, 1889, Ann. Soc. Entomol. Fr. 1889: 291 (Annam: Hue). Jacoby, 1908, Fauna India, Coleopt. 2: 299 (India).

Distribution : India, Vietnam.

This species is not included in the key. We could not check the type of this species.

Nodina similis Kimoto \& Gressitt, n. sp.

Ovate; greenish aeneous, beneath black; legs and labrum reddish brown; antenna yellowish brown with apical segments somewhat infuscate.

Head with surface strongly and closely punctate, and their interstices 
smooth and shining, vertex with a short shallow longitudinal furrow at middle; anterior margin of clypeus angularly notched. Antenna robust, short, nearly half as long as body length; first segment robust, somewhat clubshaped, nearly twice as long as wide; second slightly shorter than first and more slenderer; third and fourth slender, and subequal in length and shape to each other and nearly $2 / 3$ as long as second; fifth to tenth strongly swollen, each about as broad as long, and somewhat flattened. Pronotum nearly twice as long as wide, disc evenly covnex, smooth, and strongly and closely punctate and their interstices impressed by minute punctures. Scutellum smooth, shining, sparsely impressed by minute punctures, slightly broader than long, obtusely rounded behind. Elytron with side rounded, disc smooth, with distinct punctures in 11 rows, interstices flat, smooth, shining, impressed by minute punctures. Proepimeron with surface impunctate, granulate on basal part only.

Length $1.6-2.2 \mathrm{~mm}$.

Holotype (Type No. 2348, Kyushu Univ.), THAILAND : Songkhla, S. Thailand, 22. vi. 1965, Y. Miyatake. Paratopotypes: 44 exs., same data as the holotype (KU, BISHOP, KIMOTO). Paratypes : 17 exs., same data as the holotype but 23. vi. 1965; 19 exs., same data as the holotype but K. Morimoto; Kao Chong, nr. Trung, 8 exs., 25-27. vi. 1965, K. Morimoto \& Y. Miyatake (KU).

This new species resembles N. pusilla Motschulsky and N. malayana Bryant, but differs in having proepimeron granualte on basal part only.

\section{Nodina sphaerica Chen}

Nodina sphaerica Chen, 1940, Sinensin 11: 495 (Tonkin).

Distribution : Vietnam.

We could not check the type of this species. This species was described as new species by a single specimen. The following specimen seems to belong the same series of material described by Chen.

Material examined. Vietnam: Hoa-Binh, 1 ex., A. Cooman (PARIS).

\section{Nodina thaiensis Chî̀jô New status}

Nodina parvula thaiensis Chûjô, 1960, Nature and Life in South East Asia, Kyoto 1 : 349, fig.

4 (Thailand : Chieng Mai; CHUJO).

Distribution: Thailand.

Châjô (1961) described this species as a subspecies of parvula Jacoby, which was originally described from Burma and known to occur from Nepal also. However, thaiensis is distinctly separable from parvula in having elytron with two lateral costae in female. In parvula, elytron has no lateral costa in both sexes. We treat this species temporally as an independent species at present. 
No additional material.

\section{Genus Basilepta Baly}

Basilepta Baly, 1860 (Apr.), J. Entomol. 1: 23 (type: Basileptalongipes Baly, from Borneo). Chen, 1934, Sinensia 6 (3) : 320; 1940, ibid. 11 (5-6) : 488. —Ch ûjô, 1956, Philip. J. Sci. 85(1): 18. -Gressitt \& Kimoto, 1961, Pac. Insects Monogr. 1A: 215. -Kimoto, 1964, J . Fac. Agr. Kyushu Univ. 13(2): 244.

Nodostoma Motschulsky, 1860 (Decem.), Schrenck's Keisen Amurl. 2: 176 (first species listed : Nodostoma flavipes Motsch., Siberia). -Chapuis, 1874, Genera Coleopt. 10: 216. --Lefkvre, 1885, Mém.Soc. Sci. Liege, ser. 2, 11: 57. Jacoby, 1908, Fauna India, Coleopt. 2: 301 (type designated as fulvipes).

Mimoparascela Pic, 1935, Mel. Exot. Entomol. 65: 1 (type : Mimoparascela viridis Pic, from Tonkin). New synonymy.

Falsoiphimoides Pic, 1935, Mel. Exot. Entomol. 65: 2 (type : Falsoiphimoides bicoloripes Pic, from Tonkin). New synonymy.

\section{KEY to SPECIES of Basilepta}

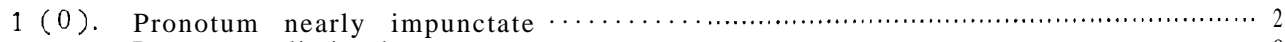

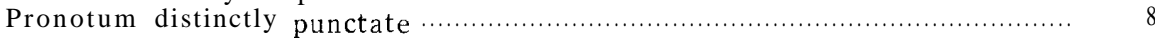

2 (1). Clypeus and anterior portion of vertex impressed by distinct punctures $\cdots \cdots \cdots$

Clypeus nearly impunctate or sparsely impressed by minute punctures,

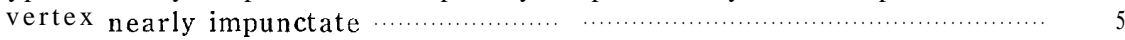

3 (2). Prothorax widest almost at base, without distinct angulation slightly

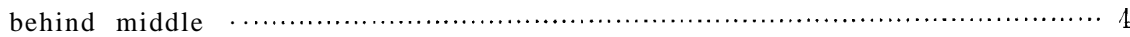

Prothorax widest sligthly behind middle and distinctly angulate; general color reddish to yellowish brown, in dark colored specimen elytron with sutural and lateral margins blackish in various degrees and in most dark colored one elytron black with apical part brownish; length

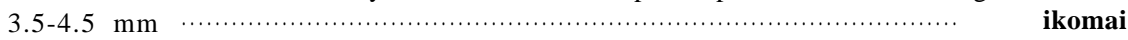

3 (3). Prothorax nearly twice as wide as long, side obtusely angulate slightly before base, and abruptly converged anteriorly; coloration variable, general color reddish brown, elytron entirely black or reddish brown with or without longitudinal stripe blackish, legs reddish brown, in dark colored specimen blackish in various degrees ; length 4.5-5.5 mm

martini

Prothorax nearly $11 / 3$ times as wide as long, side rounded, widest slightly before base, and distinctly narrowed anteriorly; general color reddish brown, in dark colored specimen elytron piceous to black in various

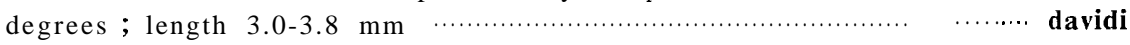

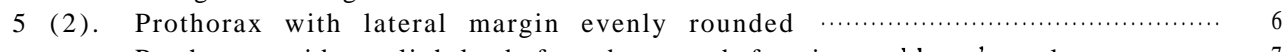

Prothorax widest slightly before base and forming subbasal angle …........... 7

6 (5). Prothorax widest almost at middle and narrowed anteriorly and posteriorly; legs reddish brown with apical half of femora and basal portion of tibiae pitchy black; general color reddish brown, antenna pitchy with basal segments brownish; length $2.4-2.7 \mathrm{~mm} \ldots \ldots \ldots \ldots \ldots \ldots \ldots \ldots$ spenceri

Prothorax widest almost at $1 / 3$ from base and strongly narrowed anteriorly and less strongly so posteriorly; general color reddish brown, antenna pitchy black with basal segments brownish; legs entirely

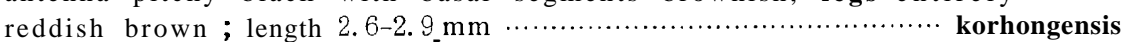



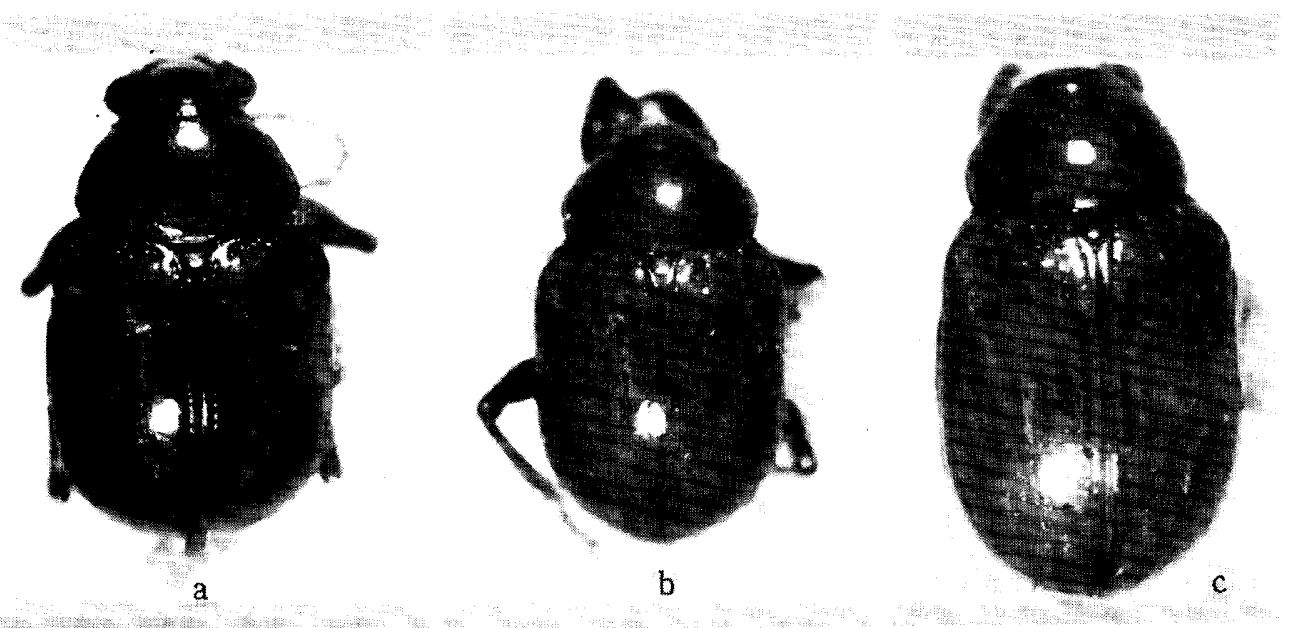

Fig. 7. a, Basilepta ikomai Chûjô ; b, B. spenceri Kimoto \& Gressitt, n. sp.; c, B. korhongensis Kimoto\& Gressitt, n. sp.

7(5). Pronotum with lateral margin widely collared at near basal angle and narrowly so anteriorly, and side abruptly coverged anteriorly in almost straight line ; entirely yellowish brown ; length 2. 6-2. $7 \mathrm{~mm}$ marginalis

Pronotum with lateral margin collared almost same width in entire margin, and side rounded, widest slightly before base and distinctly narrowed anteriorly ; coloration variable : general color reddish brown, in most dark colored specimen almost entirely pitchy black; length 2.7-3. $7 \mathrm{~mm}$

8 (1). Pronotum without smooth callosities on central portion $\ldots \ldots \ldots \ldots \ldots \ldots \ldots \ldots \ldots \ldots \ldots \ldots$

Pronotum with several conspicuous smooth callosities on central portion and sharply angulate at slightly behind middle ; elytron with dense punctures but several swollen interstices; bronzy, antenna pitchy black with four basal segments reddish brown, legs in part rufous; length $5.2 \mathrm{~mm}$......

9 (8). Prothorax somewhat hexagonal, widest near middle, with angle fairly near middle of side or at least before $1 / 3$ from base $\ldots \ldots \ldots \ldots \ldots \ldots \ldots \ldots \ldots \ldots \ldots \ldots \ldots \ldots \ldots$

Prothorax somewhat trapeziform, narrowed anteriorly, with lateral angle fairly near base, or evenly rounded

10 (9). Head with clypeus not distinctly raised; prontoum without any distinct ridge at side parallel to lateral margin

Head with clypeus convex, distinctly raised; pronotum with a distinct ridge at side parallel to lateral marign ; coloration variable : generally ochraceous to reddish or pitchy brown; length 3. '2-4. $0 \mathrm{~mm}$

11 (10). Elytron with distinct markings or with basal, lateral and sutural margins

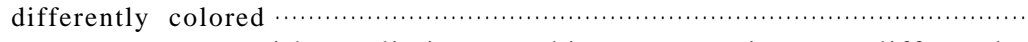

Elytron unicolor, without distinct marking or margins not differently colored

12 (11). Prothorax with lateral margin subarcuate ; elytron long, nearly three
times as long as wide, ground color of elytron brownish $\ldots \ldots \ldots \ldots . .$.
Prothorax with sharp angle slightly behind middle; elytron not especial-

12 (11). Prothorax with lateral margin subarcuate ; elytron long, nearly three
times as long as wide, ground color of elytron brownish $\ldots \ldots \ldots \ldots . .$.
Prothorax with sharp angle slightly behind middle; elytron not especial 
ly long, nearly $21 / 3$ times as long as wide; dark reddish brown, elytron black with a large median marking yellowish brown, antenna robust, especially on median segments, pitchy black with basal segments brownish, legs pitchy brown to black; length $3.8-4.8 \mathrm{~mm} \cdots \cdots$ miyatakei

13 (12). Pronotum sparsely impressed by minute punctures ; elytron yellowish brown with basal, lateral and sutural margins pitchy black; yellowish to reddish brown, antenna brownish with apical segments blackish, legs mostly blackish ; length $5.0 \mathrm{~mm}$

longipennis

Pronotum distinctly and closely impressed with large punctures which are much larger than average elytral punctures and separated by less than their diameters; ground color reddish brown, elytron with basal and postmedian markings blackish, in most dark colored specimen basal half of sutural, interior half of basal and subbasal part of lateral margins and part of postbasal transverse impression together with a pair of markings of pronotum and ventral surfaces of thorax blackish;

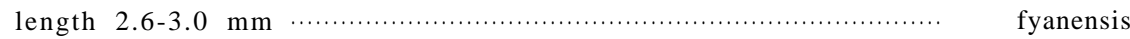

14 (11). Dorsum unicolor, entirely pale or dark reddish to yellowish brown $\cdots \cdots \cdots \cdots \cdots \cdots$

Dorsum not entirely brownish, at least partly blackish, bluish, violace-

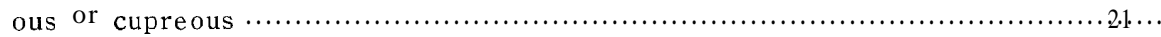

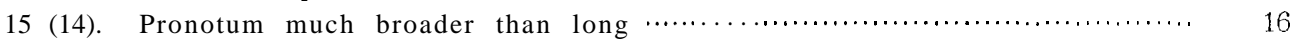

Pronotum as long as wide, strongly and somewhat closely punctate ; general color deep red, side and base of pronotum dark brown, antenna reddish brown with apical segments infuscate, legs pitchy brown with basal portion of femora paler; length $3.5 \mathrm{~mm} \ldots \ldots \ldots \ldots \ldots \ldots \ldots \ldots \ldots \ldots+$ gracilis

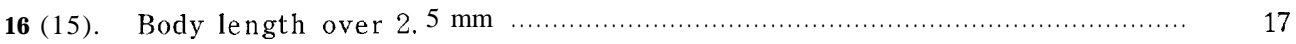

Body length shorter; prothorax widest almost at middle and strongly narrowed anteriorly and posteriorly, surface distinctly and homogeneously impressed by large punctures which are much larger than average elytral punctures and separated by approximately their diameters ; general color pitchy brown; length 1. $8-1.9 \mathrm{~mm}$.................. minutissima

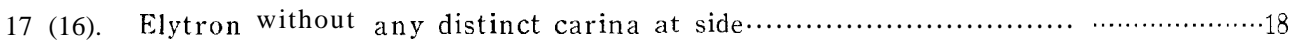

Elytron with a distinct carina, starting from humerus and ending nearly middle of side; prothorax widest slightly behind middle and surface distinctly impressed with large punctures especially on lateral part; yellowish brown, with top of vertex infuscate; length $3.7-4.0 \mathrm{~mm} \cdots \cdots . .$. maai

18 (17). Prothorax about $11 / 2$ times as broad as long, surface distinctly punctate

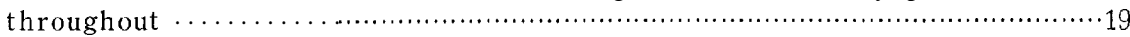

Prothorax nearly twice as broad as long and side not very obtusely angulate below middle, surface fairly strongly punctate in middle but obsolete at side and anterior collar very deeply set off; yellowish

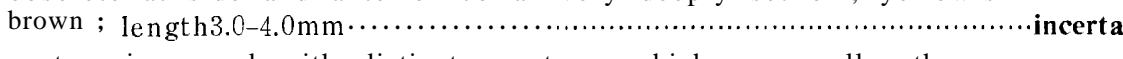

19 (18). Pronotum impressed with distinct punctures which are smaller than or subequal to average elytral punctures and separated by more than their diameters

Pronotum distinctly and closely impressed by large punctures which are much larger than average elytral punctures and separated by less than their diameters; reddish brown, antenna pitchy black with basal segments brownish, ventral surfaces brownish with lateral portion of thorax in part blackish; legs blackish with middle of femora reddish

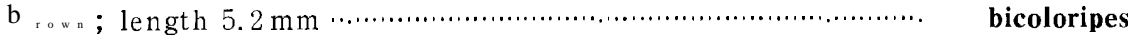

20 (19). Body length larger, elytral puncture rows largely continuing behind basal $1 / 3$; pronotum strongly and fairly closely punctate; general color 
reddish brown, with basal margin pitchy black; legs piceous with basal half of femora and apical part of tibiae and entire tarsi paler; length $3.5 \mathrm{~mm}$

melanopus

Body length smaller, elytral puncture rows largely obliterated behind basal $1 / 3$; pronotum distinctly but closely punctate; yellowish brown ;

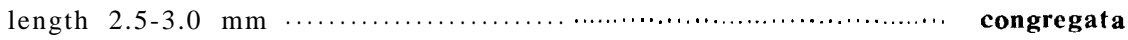

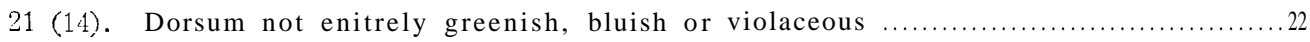

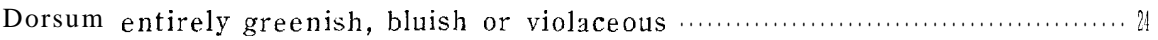

22 (21). Prothorax with anterior sulcus not entire but distinct only on lateral

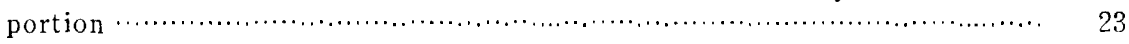

Prothorax with anterior sulcus entire; general color cupreous with purple or greenish luster; antenna pitchy black with basal segments dark reddish brown; legs dark cupreous with tibiae and tarsi dark reddish brown; length $4.0 \mathrm{~mm}$......

23 (22). Prothorax with sharp angle almost at middle; general color black; head, prothorax and scutellum reddish brown; antenna pitchy black with basal segments brownish ; legs shining black with most of median portion of femora reddish brown; length $5.0 \mathrm{~mm} \ldots \ldots \ldots \ldots \ldots \ldots \ldots \ldots$ semirufa

Prothorax with sharp angle slightly behind middle; prothorax blackish with anterior margin slightly pitchy; head pitchy black with middle of vertex brownish, elytron reddish brown, antenna pitchy black with basal segments brownish, legs and ventral surfaces of thorax pitchy black and abdomen dark reddish brown; length $3.4 \mathrm{~mm}$.............. morimotoi

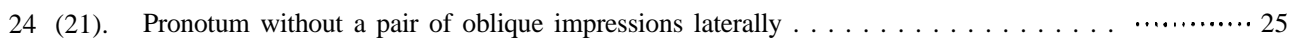

Pronotum with a pair of oblique impressions laterally, and its suface finely granulate; elytron with a short but sharply raised humeral ridge; general color greenish; antenna pitchy black with basal seg-

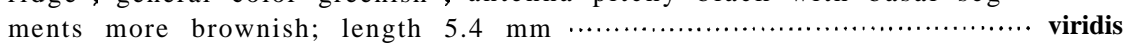

25 (24). Prothorax with obtuse angle near at middle of side . . . . . . . . ............. 26

Prothorax with sharp angle slightly behind middle of side; bluish black

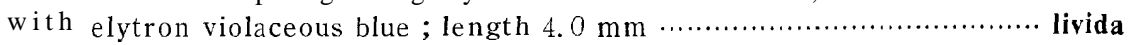

26 (25). Elytron with one or more distinct carinae or ridges at side $\ldots \ldots \ldots \ldots \ldots \ldots 27$

Elytron without ridge at side; antenna blackish with basal segments somewhat brownish; legs entirely violaceous blue; general color violaceous blue; length $5.0-6.0 \mathrm{~mm}$

27 (26). General color violaceous blue, elytron with two carinae and a tubercle

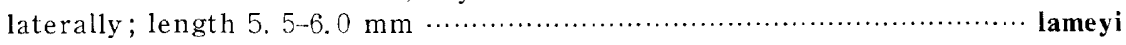

General color bluish black, pronotum greenish blue, elytron violaceous blue with humerus greenish; elytron with a distinct carina laterally;

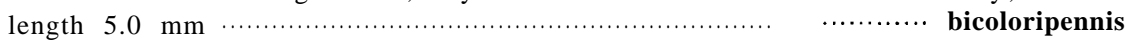

28 (9). Head finely or distinctly punctate and interstices of punctures smooth, not granulate

Head coarsely and strongly punctate and interstices of punctures finely granulate; pronotum distinctly and rather closely punctate, and with collar deeply set off by a row of punctures; prothorax with side distinctly angulate near base, but in some specimen obtuse or rounded; metallic green, violaceous, bluish or blackish; legs reddish brown with tarsi and part of tibiae blackish, but in dark colored specimen entire-

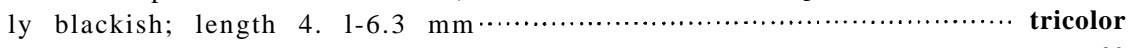

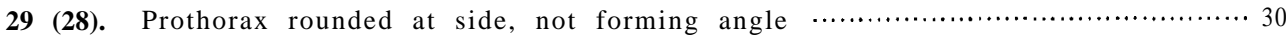

Prothorax forming an angle posterolaterally, which is obtuse or sharp ….... 37

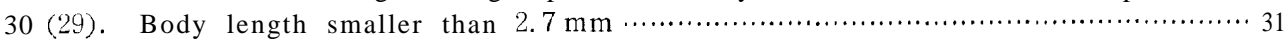




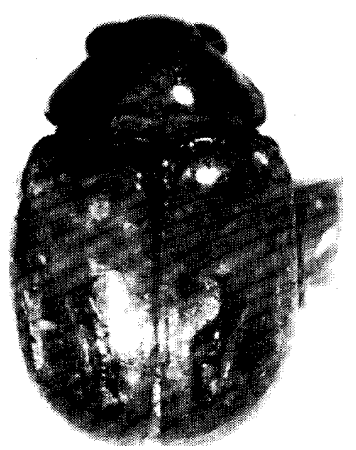

a

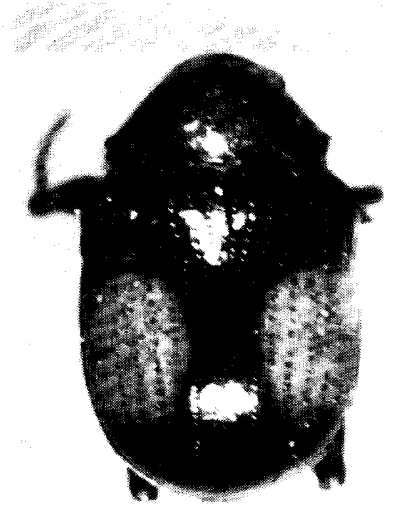

b.

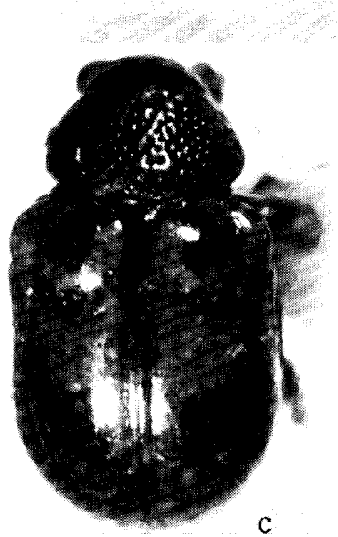

C

Fig. 8. a, Basilepta marginalis Kimoto \& Gressitt, n. sp.; b, B. miyatakei Kimnto \& Gressitt, n. sp.; c, B. fyanensis Kirnoto \& Gressitt, n. sp.

Body length larger than $5.0 \mathrm{~mm}$

31 (30). Pronotum with anterior sulcus distinct on lateral portion and shallower at middle and impressed by a row of distinct punctures; antenna robuster, in preapical segments nearly twice as long as wide; dorsal surfaces greenish blue; ventral surfaces black; antenna pitchy black with basal segments brownish ; legs entirely pitchy black; length 2. 7 $\mathrm{mm}$

viridicyanea

Pronotum with anterior sulcus distinct only on lateral portion and interrupted at middle; antenna slenderer, in preapical segments nearly three times as long as wide; head, thorax and sutural and lateral margins of elytron metallic green, disc of elytron reddish brown to deep red; antenna and legs yellowish to reddish brown; ventral surfaces metallic green ; pronotum disinctly but not closely punctate; transverse depression behind subbasal area of elytron obsoletely impressed ; length $2.3-2.5 \mathrm{~mm}$......

$32(30)$. Elytron unicolor, without distinct marking 33

Elytron yellowish brown with large basal and smaller subapical markings blackish, general color yellowish brown, with head and ventral surfaces of thorax largely blackish, pronotum sparsely impressed with minute punctures ; length $5.0 \mathrm{~mm}$

33 (32). Elytron without ridge at side, dorsum not entirely violaceous blue $\ldots \ldots \ldots \ldots \ldots . \cdots 34$

Elytron with weak ridge at side, partly rough behind at middle; antenna fulvo-testaceous with five apcial segments fuscous; general color obscure cyaneous; length $5.0-5.5 \mathrm{~mm} \ldots \ldots \ldots \ldots \ldots \ldots \ldots \ldots \ldots \ldots \ldots \ldots \ldots \ldots \ldots . \ldots \ldots$ fabrei

34 133). Dorsum reddish cupreous, with greenish luster especially on lateral part $\ldots \ldots . . .35$ Prothorax reddish brown, elytron bluish or blackish . . . . . . . .................... 36

35 (34). Pronotum sparsely impressed by distinct punctures on middle and more closely so laterally; ventral surfaces reddish brown with greenish luster on thorax; legs reddish brown; length $5.0 \mathrm{~mm} \ldots \ldots \ldots \ldots \ldots \ldots \ldots$ speciosa

Pronotum closely impressed with distinct punctures and more closely so laterally, interstices of punctures nearly as wide as diameters of punctures; ventral surfaces dark reddish brown with thorax dark to 
blackish brown; legs reddish brown; length $4.6-6.7 \mathrm{~mm}$................ rondoni

36 (34). Elytron black; head and thorax reddish brown; general color black; pronotum sparsely impressed by distinct punctures; length $7.0 \mathrm{~mm} \cdots$ latipennis

Elytron greenish blue; head and thorax reddish brown; general color black; pronotum sparsely impressed with distinct punctures ; length

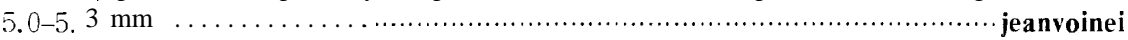

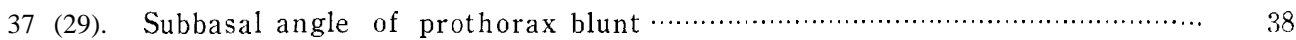

Subbasal angle of prothorax sharp; elytron with a sharply raised ridge, running parallel with lateral margin to about middle; general color fulvus or piceous, elytron metallic green or blue, in most pale colored specimen dorsum entirely fulvus and in most dark colored one dorsum entirely bluish ; length $3.0-3.7 \mathrm{~mm}$

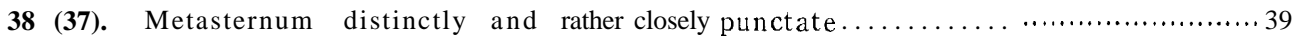

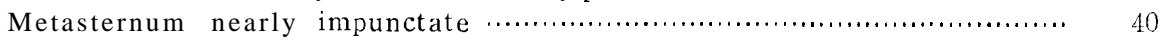

39 (38). Lateral margin of prothorax abruptly converging anteriorly in almost straight line; pronotal punctures smaller than average elytral punctures and finely and more closely impressed on lateral part and more sparsely and finely so on median and anterior parts, and those separated by more than diameters even in lateral part; coloration variable : reddish, brownish, bluish, greenish, violaceous or blackish ;

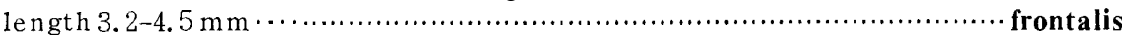

Lateral margin of prothorax less abruptly and rather convexly converging anteriorly; pronotal punctures larger than, or subequal to average elytral punctures, and distinctly and almost homogeneously impressed, except for anterior part, and those separated by approximately, or less than their diameters; coloration variable : reddish, brownish, greenish, violaceous, bluish or blackish ; length $3.7-4.3 \mathrm{~mm}$........... puncticoliis

40 (38). Pronotal punctures finely and not so closely impressed, and much smaller than average elytral punctures; prothorax with lateral margin widely collared apically, and width of collar subequal to width of apical segments of antenna; yellowish brown, in some specimen, postscutellar part of sutural margin and lateral margin of elytron blackish, and in most dark colored specimen almost entirely pitchy black; length 2.9-

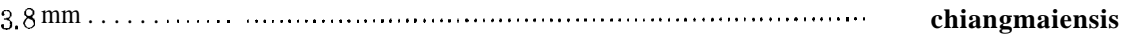

Pronotal punctures distinctly and closely impressed, and larger than, or subequal to average elytral punctures ; prothorax with lateral margin widely collared apically, and width of collar much wider than the width of apical segments of antenna; dark yellowish brown, pronotum with a pair of large median markings pitchy black, elytron with basal, laterobasal, postmedian and lateromedian markings pitchy black; ventral surfaces mostly pitchy black and apical segments of abdomen partly brownish ; legs with apex of femora and base of tibiae blackish; length $4.5-4.8 \mathrm{~mm}$ multimaculata

\section{Basilepta bicolor (Lefèvre) New combination}

Nodostomabicolor Lef., 1893, Ann. Soc. Entomol. Fr. 62: 118 (Tonkin: Lang-Son).

Nodostoma bicolor var. submarginatum Pic, 1930, Mél. Exot. Entomol. 56: 7 (Tonkin).

Distribution : Vietnam .

We could not trace the type location of this species. This species is not included in the key. 


\section{Basilepta beccarii (Jacoby) (Fig. 2a)}

Nodostoma beccarii Jac., 1884, Ann. Mus. Civ. Genova 20: 214 (Java). -Lefèvre, 1889, Ann

Soc. Entomol. Fr., ser. 6, 9: 233 (Saigon) ; 1833, ibid. 1893: 118 (Tonkin).

Basilepta beccarii : Chen, 1935, Sinensia F(3): 337 (Tonkin, Java, Sinagar).

Distribution : Thailand, Laos, Vietnam, Java.

Material ExAmined. THAILAND : Uthaithani, 2 exs., 13. iv. 1963 (BANGKHEN) ; Chiangmai Prov., Chiangmai (Arboretum), 300 m, 1 ex., 12. vi. 1965, P. D. Ashlock; Chiangmai, 1 ex., 9. vi. 1965, P. D. Ashlock; Trang Prov., Khaophappha, Kaochang, 200-400 m, 1 ex., 11. i. 1964, G. A. Samuelson (BISHOP) ; Chieng Dao, 1 ex., 15. vi. 1965, K. Morimoto; Chiangmai, 1 ex., 12. vi. 1965, K. Morimoto; Botanical Garden, Chiangmai, 1 ex., 12. vi. 1965, Y. Miyatake ; Khao Yai Nat. Park, 2 exs., 6. vi. 1965, K. Morimoto \& Y. Miyatake; Khao Chong, nr. Trang, 1 ex., 27. vi. 1965, Y. Miyatake (KU). LAOS: Ban Van Heue, $20 \mathrm{~km}$ E of Phou-kow-kuei, 1 ex., l-15. v. 1965, J. A. Rondon (BISHOP). VIETNAM: Ban Me Thuot, $500 \mathrm{~m}, 2$ exs., 16-18. v. 1960, L. W. Quate; $7 \mathrm{~km}$ SE of Dilinh (Djiring), 990 m, 3 exs., 2. v. 1960, R. E. Leech; Fyan, 900-1000 m, 1 ex., 11. vii. -9. viii. 1961, N. R. Spencer; $25 \mathrm{~km} \mathrm{SE} \mathrm{of} \mathrm{Pleiku,} 400 \mathrm{~m}, 1$ ex., 12. v. 1960, L. W. Quate ; Mt. Lang Bian, 1500 m-2000 m, 1 ex., 19. v. -8. vi. 1961, N. R. Spencer (BISHOP) ; Hoa-Binh, Tonkin, 2 exs., A. Cooman (FREY).
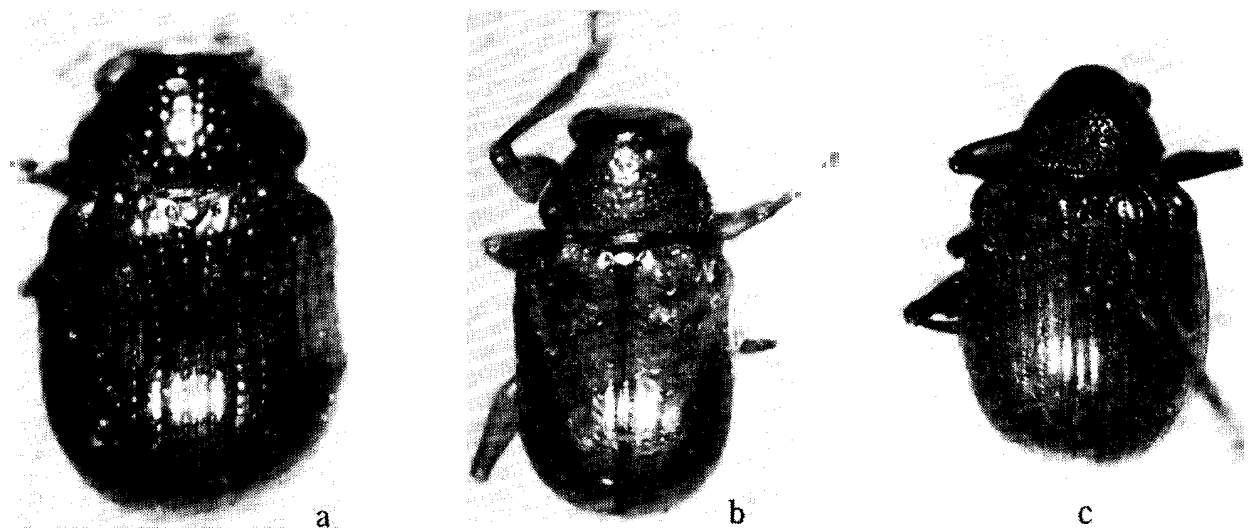

Fig. 9. a, Basileptaminutissima Kimoto \& Gressitt, n. sp.; b, B. maai Kimoto \& Gressitt, n. sp.; c, B. bicoloripes (Pic), holotype.

\section{Basilepta bicoloripennis $(\mathrm{Pic}) \mathrm{New}$ combination}

Nodostoma bicoloripenne Pic, 1930, Mél. Exot. Entomol. 56: 7 (Tonkin; PARIS).

Distribution : Vietnam.

No additional material. This species might be prooved as an infraspecific variation of B.lameyi (Lefèvre) in future. 
Basilepta bicoloripes (Pic) New combination (Fig. 9c)

Falsoiphimoides bicoloripes Pic, 1935, Mél. Exot. Entomol. 65 :2(Tonkin; PARIS).

Distribution : Vietnam.

No additional material.

\section{Basilepta binhana (Pic) New combination}

Nodostoma binhanumPic, 1930, Mél. Exot. Entomol. 56: 8(Tonkin: Hoa-Binh ; PARIS).

Basileptadistinctum Chen, 1935, Sinensia 6(3):314 (Tonkin; PARIS). -Gressitt \& Kimoto, 1961, Pac. Insects Monogr. 1A:220(Tonkin, Hainan, S. China). N ew synonymy.

Distribution : S. China, Hainan, Vietnam.

Material examined. VIETNAM: Hoa-Binh, 5 exs., A. Cooman (FREY) ; 9 $\mathrm{km} \mathrm{S}$ of Dilinh (Djiring), 1 ex., 24. iv. 1960, R. E. Leech; $31 \mathrm{~km} \mathrm{~S}$ of Dilinh (Djiring), 1050 m, 1 ex., 29. iv. 1960, on grass near bamboo, R. E. Leech (BISHOP).

\section{Basilepta chapaensis (Pic) New combination}

Nodostoma ckapaense Pic, 1930, Mél. Exot. Entomol. 56: 6(Tonkin:Chapa; PARIS).

Distribution: Vietnam.

No additional material.

Basilepta chiangmaiensis Kimoto \& Gressitt, n.sp. (Fig. 13a)

Body oblong oval; general color yellowish brown, in some specimen postscutellar part of sutural margin and lateral margin of elytron blackish, and in most dark colored specimen almost entirely pitchy black.

Head distinctly and rather closely punctate, vertex with a short longitudinal furrow at middle. Antenna filiform, nearly $2 / 3$ as long as body length; first segment robust, somewhat club-shaped ; second shortest, robuster, nearly half as long as first; third slenderer, nearly $11 / 3$ times as long as second; fourth $1 \frac{1 / 2}{2}$ times as long as third ; fifth slightly longer than fourth; sixth and seventh subequal to fifth in length and shape; eighth slightly shorter than seventh; ninth and tenth subequal to eighth in length and shape; eleventh slightly longer than tenth and its apex pointed. Pronotum transverse,

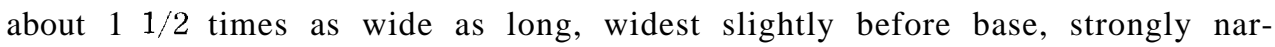
rowed anteriorly and subbasal angle rather blunt; anterior angle somewhat denticulate and with a setigerous puncture, and basal angle slightly tuberculate and with a setigerous puncture; anterior margin distinctly rounded anteriorly, and posterior margin widely rounded posteriorly, lateral margin widely collared apically, and width of collar subequal to width of apical segments of antenna; dorsal surface finely and not so closely punctate and diameter of punctures much smaller than average elytral punctures, anterior sulcus entire and impressed with a row of distinct punctures; preopimeron wrinkled, im- 
punctate; mesosternum nearly impunctate, sparsely covered with fine pubescence. Elytron subparallel-sided with apex rounded ; subbasal area slightly convex and separated from behind by a distinct transverse furrow; punctures regularly arranged in longitudinal rows and these punctures generally larger and deeper and even distinct on apical portion.

Length 2.9-3.8 $\mathrm{mm}$.

Holotype (Type No. 2349, Kyushu Univ.), THAILAND: Doi Pui, $1300 \mathrm{~m}$, Chiang Mai Prov., 8. vi. 1965, Y. Miyatake. Paratopotypes: 7 exs., same data as the holotype (KU, BISHOP, KIMOTO). Paratypes : THAILAND : 7 exs., same data as the holotype but 17. vi. 1965, Doi Suthep, $1000 \mathrm{~m}$, Chiang Mai Prov., 1 ex., 8. vi. 1965, K. Morimoto, 1 ex., same data, Y. Miyatake, 1 ex., 10. vi. 1965, K. Morimoto, 2 exs., 18. vi. 1965, K. Morimoto, 1 ex., same data, Y. Miyatake (KU) ; Mae Sa, Chiang Mai Prov., 1 ex., 16. vi. 1965, P. D. Ashlock (BISHOP) ; LAOS: Ban Van Heue, $20 \mathrm{~km} \mathrm{E}$ of Phou-Kow-Kuei, 1 ex., 1 15. v. 1965, J. A. Rondon (BISHOP) ; VIETNAM: Fyan, $1200 \mathrm{~m}, 2$ exs., 11. vii. -1. viii. 1961, N. R. Spencer ; Mt. Lang Bian, 1500-2000 m, 6 exs., 19. v. -8. vi. 1961, N. R. Spencer; Dilinh (Djiring), $1200 \mathrm{~m}, 3$ exs., 22-28. iv. 1960, S. Quate \& L. W. Quate; $20 \mathrm{~km} \mathrm{~N}$ of Pleiku, $600 \mathrm{~m}, 1$ ex., 9. 1960, S. Quate; Dai Lanh, N of Nha Trang, 1 ex., 30. xi. -5. xii. 1960, C. M. Yoshimato ; Dalat, $1500 \mathrm{~m}, 2$ exs., 28. iv. -4. v. 1960, S. Quate; Blao (Balao), 500 m, 1 ex., 14-21. v. 1960, C. M. Yoshimoto (BISHOP).

Distribution : Thailand, Laos, Vietnam.

This new species somewhat resembles Basilepta yimnae Gressitt \& Kimoto, from S. China, but differs in being body length shorter, and having the anterior margin of prothorax more strongly rounded anteriorly.

\section{Basilepta congregata (Jacoby) (Fig. 10a)}

Nodostoma congregatum Jac., 1908, Fauna India, Coleopt. 2: 329 (India: Travancore; BM). Basilepta congregatum: Gressitt \& Kimoto, 1961, Pac. Insects Monogr. 1A: 219 (Hainan).

Distribution : India, Thailand, Laos, Vietnam, Hainan.

Considering about the variation of this species, especially on the degrees of the lateral angulation of pronotum and pronotal punctures, it would be possible that Basilepta incertum $\mathrm{Pic}(=$ pallicorne Chen) might be a synonymy of this species.

Material ExAmined. THAILAND: Nakhon Nayok Prov., Khao Yai Nat. Park, 2 exs., 5. vi. 1965, P. D. Ashlock; Chiangmai Prov., Fang, 500 m, 1 ex., 13. vi. 1965, P. D. Ashlock; Tang Keo, 1 ex., 10. vi. 1965. P. D. Ashlock; Doi Suthep, 1000 m, 1 ex., 12. vi. 1965. P. D. Ashlock (BISHOP) ; Doi Suthep, 1000 m, 8 exs., 12. vi. 1965, 31 exs., 17-19. vi. 1965, K. Morimoto \& Y. Miyatake; Fang, 1 ex., 14. vi. 1965, K. Morimoto, 1 ex., 20. viii. 1973, K. Yano ; Chieng Dao, 4 exs., 15. vi. 1965, K. Morimoto \& Y. Miyatake; Khao Yai Nat. Park, 15 exs., 5-6. vi. 1965, K. Morimoto \& Y. Miyatake; Pak Chong, Korat Plateau, 1 ex., 14. iv. 
1973, Y. Yoshiyasu (KU). LAOS: Ban Van Heue, $20 \mathrm{~km}$ E of Phou-kow-kuei, 1 ex., 1-15. v. 1965, J. A. Rondon; Borikhane Prov., Paksane, 1 ex., 8. xii. 1965, Native collr (BISHOP). VIETNAM: Dilinh (Djiring), $1200 \mathrm{~m}, 3$ exs., 2228. iv. 1960, S. Quate \& L. W. Quate; Ap. Hung-Lam, $21 \mathrm{~km} \mathrm{NW}$ of Dilinh, 1100 m, 1 ex., 29. ix. - 5. x. 1960, C. M. Yoshimoto; Dalat, 1500 m, 4 exs., 29. iv. -4 . v. 1960, L. W. Quate ; $15 \mathrm{~km} \mathrm{NW}$ of Dalat, n850 ex., 5. v. 1960, L. W. Quate; Mt. Lang Bian, 1500-2000 m, 4 exs., 19. v. -8. vi. 1961, N. R. Spencer ; Blao (Balao), 500 m, 1 ex., 14-21. x. 1960, C. M. Yoshimoto; Dak Song, $76 \mathrm{~km}$ SW of Ban Me Thuot, $870 \mathrm{~m}, 1$ ex., no date, R. E. Leech (BISHOP).

\section{Basilepta davidi (Lefèvre)}

N odostoma davidi Lef., 1877, Ann. Soc. Entomol. Fr. ser. 5, 7: 157 (China; PARIS).

Nodostoma atriventre Pic, 1933, Mel. Exot. Entomol. 61: 7 (Tonkin; PARIS) ; 1934, ibid. 64: 7 (Tonkin). New synonymy.

Nodostoma atriventre var. approximatum Pic, 1933, Mel. Exot. Entomol. 61: 8 (China) ; 1934, ibid. 64: 7 (China).

Nodostoma okinawense Chûjô, 1935, Trans. Nat. Hist. Soc. Formosa 25: 77 (Ryukyu Is. ; TARI); 1956, Philip. J. Sci. 85(1) : 28 (=davidi).

Nodostoma insulanum Chûjô, 1935, Trans Nat. Hist. Soc. Fromosa 25: 204 (Ryukyu Is.; TARI) ; 1956, Philip. J. Sci. 85(1): 28 (=davidi).

Basilepta davidi: Chen, 1935, Sinensia 6 (3) : 306 (China) ; 1940, ibid. 11 (5-6): 484 (host: Populus). - Chûjô, 1956, Philip. J. Sci. 85 (1) : 28 (Formosa). -Gressitt \& Kimoto, 1961, Pac. Insects Monogr. 1A: 219 (China, Hainan, Korea). -Kimoto, 1964, J. Fac. Agr. Kyushu Univ. 13(2): 245, 248 (Japan, Ryukyu Is.).

Basilepta atriventris approximata: Chen, 1940, Sinensia 11 (5-6) :502(China).

Distribution: Vietnam, Hainan, Taiwan, China, Ryukyus, Korea.

No additional material.

\section{Basilepta fabrei (Lefèvre) New combination}

Nodostoma fabrei Lef., 1887, Bull. Soc. Entomol. Fr., 1887: 175 (Hindustan : Rammand) ; 1893, Ann. Soc. Entomol. Fr. 1893: 118 (Tonkin). -Jacoby, 1908, Fauna India, Coleopt. 2: 308 (India, Burma).

Distribution : India, Burma, Vietnam.

We could not trace the type location of this species. No additional material.

\section{Basilepta frontalis (Baly) New combination (Fig. 12b)}

Nodostoma aeneipenne Baly, 1867 (nec Motschulsky, 1860), Trans. Entomol. Soc. Lond., ser. 3, 4(2): 235 (Malacca ; BM). - Jacoby, 1884, Notes Leyd. Mus. 6: 14 (Sumatra). -Lefèvre, 1889, Ann. Soc. Entomol. Fr., ser. 6, 9: 295 (Tonkin : Lien-Son) ; 1890, Nouv. Arch. Mus. Paris, ser. 3, 2: 193 (Siam) ; 1904, Miss. Pavie Indo-China, ser. 2, 3 (2001) : 149 (Siam).

Nodostoma frontale Baly, 1867, Trans. Entomol. Soc. Lond., ser. 3, 4(2): 253 (Malacca ; BM). -Lefèvre, 1893, Ann. Soc. Entomol. Fr. 1893: 118 (Tonkin).

Nodostoma apicipes Jacoby, 1892, Entomologist 25 (Suppl.) : 87 (Sumatra, Burma; BM) ; 1908, Fauna India, Coleopt, 2: 331 (=aeneipenne). 
Nodostoma haroldi Jacoby, 1908, Fauna India, Coleopt. 2: 331 (new name for aeneipenne Baly, nec Motschulsky, 1860, from Dauria). New synonymy.

Nodostoma occipitale Jacoby, 1908, Fauna India, Coleopt. 2: 335 (Burma: Tenasserim; BM). New synonymy.

Basilepta haroldi: Gressitt \& Kimoto, 1961, Pac. Insects Monogr. 1A: 224 (Hainan).

Basilepta occipitale:Chûjô, 1964, Nature and Life in SE Asia, Kyoto 3: 268 (Thailand : $\mathrm{Na}$ born)

Distribution : India, Burma, Thailand, Laos, Vietnam, Hainan, Malaya, Sumatra.

Material examined. Thailand: Trang Prov., Khaophappha, Kaochang, 200-400 m, 2 exs.. 9. i. 1964, 3 exs., 11. i. 1964, 1 ex., 13. i. 1964, G. A. Samuelson; Banna, Chawang, nr. Nabon, 1 ex., 4. ix. 1958, J. L. Gressitt; Chiang Mai Prov., Fang (Agr. Exp. Station), 600 m, 2 exs., 14. vi. 1965, P. D. Ashlock; Maesa, 1 ex., 16. vi. 1965, P. D. Ashlock (BISHOP) ; Fang, 7 exs., 13. vi. 1965, 26 exs., 14. vi. 1965, K. Morimoto, 2 exs., 14. vi. 1965, Y. Miyatake; Mae Sa Water Fall, nr. Chom Thong, 1 ex., 11. vi. 1965, 3 exs., 16. vi. 1965, K. Morimoto; Tankeo, Doi Suthep, $800 \mathrm{~m}, 1$ ex., 10. vi. 1965, Y. Miyatake; Kao Yai Nat. Park, 2 exs., 6. vi. 1965, Y. Miyatake; Khao Chong nr. Trung, 8 exs., 25-26. vi. 1965, K. Morimoto \& Y. Miyatake (KU). LAOS: Umgeb. Vientiane, 1 ex., iii-vi. 1963; Umgeb. Vanky, 2 exs., 1963; Umgeb. Paklay, 2 exs., 1963 (MUNCHEN) ; Ban Van Heue, $20 \mathrm{~km} \mathrm{E}$ of Phou-kow-kuei, 2 exs., 1-15. v. 1965, J. A. Rondon, 4 exs., 15-31. v. 1965, Native collr; Attopeu Prov., Houei Kong, 1 ex., 31. v. 1965, Native collr ; Borikhang Prov., Pakkading, 1 ex., 9. ix. 1965, Native collr (BISHOP). VIETNAM: Ban Me Thuot, $500 \mathrm{~m}, 2$ exs., 16-18. v. 1960, S. Quate \& L. W. Quate; $22 \mathrm{~km} \mathrm{~S}$ of Nha Trang, 3 exs., 20-26. xi. 1960, C. M. Yoshimoto; Kontum, N of Pieiku, 550 m, 1 ex., 13. v. 1960, L. W. Quate (BISHOP).

Basilepta fyanensis Kimoto \& Gressitt, n. sp. (Fig. 8c)

Body oblong oval; ground color reddish brown, elytron with basal and postmedian markings blackish, in most dark colored specimen basal half of sutural, interior half of basal and subbasal part of lateral margins and part of postbasal transverse impression, together with a pair of markings of pronotum and entire ventral surfaces of thorax blackish; antenna pitchy black with three or four basal segments reddish brown but in most pale colored specimen entirely reddish brown; legs entirely reddish brown.

Head with surface smooth, strongly and closely punctate, clypeus with a pair of distinctly raised short longitudinal costae laterally. Antenna filiform, long, nearly $3 / 4$ as long as body length; first segment robust, somewhat clubshaped; second nearly $3 / 5$ as long as first, and nearly $21 / 2$ times as long as wide ; third nearly $11 / 3$ times as long as second, and much slenderer; fourth $11 / 2$ times as long as third; fifth to tenth subequal to fourth in length but graudally widened toward apical segments ; eleventh nearly $1 \frac{1 / 3}{3}$ times as 


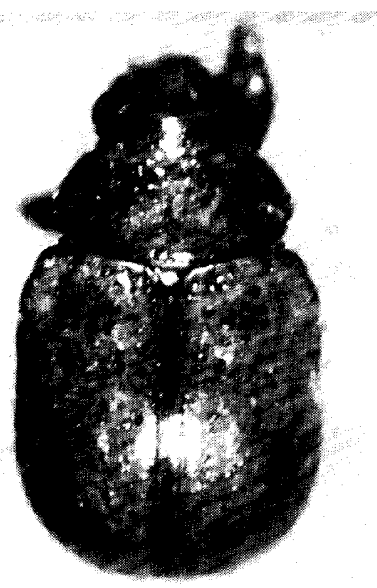

a

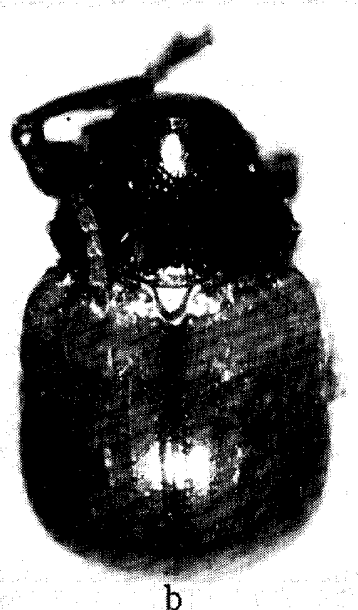

$\mathrm{b}$

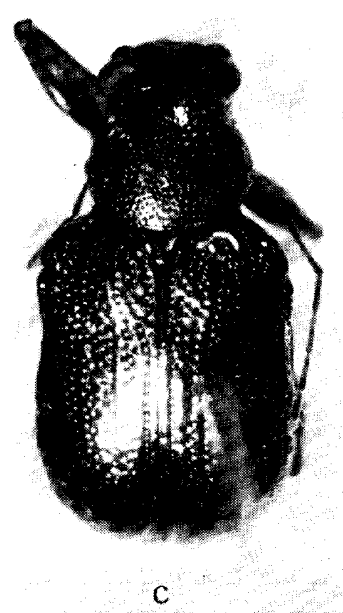

c

Fig. 10. a, Basilepfa congrega a (Jacoby); b, B. morimotoi Kimoto \& Gressitt. n. sp.; c, B. viridis Pic, holotype.

long as tenth and its apex pointed. Pronotum transverse, nearly $11 / 2$ times as long as wide, widest almost at middle, lateral margin subarcuate, not sharply produced laterally, and distinctly narrowed anteriorly and posteriorly; anterior margin distinctly rounded anteriorly and posterior margin rounded and slightly produced posteriorly, anterior corner somewhat denticulate and with a setigerous puncture and posterior corner sligthly tuberculate and with a setigerous puncture; dorsal surface distinctly and closely impressed with large punctures which are much larger than average elytral punctures and separated by less than their diameters, interstices of punctures smooth, shining and anterior sulcus distinct on lateral portion and shallower at middle, and impressed by a row of distinct punctures; proepimeron nearly smooth, rather closely impressed by large punctures; metasternum very sparsely covered with fine hairs and minute punctures. Scutellum subtrigonate, with apex rounded; surface slightly convex, smooth, shining, nearly impunctate. Elytron relatively long, subparallel-sided with apex rounded ; subbasal area distinctly raised and separated from behind by a deep transverse furrow; punctures regularly arranged in longitudinal rows, and these punctures stronger and deeper at subbasal transverse furrow and finer at subbasal and median portion and obsolescent at apical portion; humerus distinctly raised and with a short but distinct costa starting from humerus.

Length 2.6-3.0 mm.

Holotype (BISHOP), VIETNAM: Fyan, 900-1000 m, 11. vii. -9. viii. 1961, N. R. Spencer. Paratopotypes : 2 exs., same data as the holotype (BISHOP, KIMOTO) . Paratypes : VIETNAM: Dalat, 1500 m, 2 exs., 29. iv. -4. v. 1960, L. W. Quate (BISHOP). 
This new species somewhat resembles Basileptamaai Gressitt \& Kimoto, but differs in being the body length shorter, and having the punctures of pronotum larger and closer.

\section{Basilepta gracilis $\boldsymbol{C}$ he $\mathrm{n}$}

Basilepta gracile Chen, 1935, Sinensia 6(3): 317, fig. 22 (Tonkin ; PARIS). -Gressitt \& Kimoto, 1961, Pac. Insects Monogr. 1A: 224 (Tonkin).

Distribution : Vietnam.

No additional material.

Basilepta ikomai Châjồ (Fig. 7a)

Basilepta ikomai Chûjô, 1961, Nature and Life in SE Asia, Kyoto 1: 347, fig. 2 (Thailand: Chieng Mai; CHUJO) ; 1964, ibid, 3 : 267 (Thailand : Chieng Mai).

Basilepta nigrofasciatum : Chûjô, 1961, Nature and Life in SE Asia, Kyoto 1: 348 (Thailand: Chieng Mai); 1964, ibid. 3: 268 (Thailand : Mae Fack).

Distribution : Thailand, Cambodia, Laos.

Material EXAmined. THAILAND: Nakorn-nayok, 1 ex., 14. v. 1963 (BANGKHEN) ; Tang Prov., Kahophappha, Kaochang, ZOO-400 m, 3 exs., 2-4. i. 1964, G. A. Samuelson ; Chiangmai Prov., Fang (Agr. Exp. Station), 600 m, 2 exs., 14. vi. 1965, P. D. Ashiock (BISHOP) ; Fang, 4 exs., 13-14. vi. 1965, K. Morimoto \& Y. Miyatake; Doi Suthep, 1000 m, Chiang Mai Prov., 2 exs., 12. vi. 1965, K. Morimoto; Khao Yai Nat. Park, 4 exs., 6. vi. 1965, S. Asahina, K. Morimoto \& Y. Miyatake; Khao Chong, nr. Trung, 9 exs., 25-27. vi. 1965, K. Morimoto \& Y. Miyatake (KU). CAMBODIA: Bamrey Phong, 2 exs., 14-16. iv. 1961, N. R. Spencer (BISHOP). LAOS: Vientiane, 2 exs., 8-9. v. 1965, P. D. Ashlock; Muong Sing, NW of Luang Prabang, $650 \mathrm{~m}, 1$ ex., 6-10. vi. 1960, L. W. Quate (BISHOP).

\section{Basilepta incerta (Pic) New combination}

Pagria incerta Pic, 1928, Mél. Exot. Entomol. 53: 33 (Tonkin; PARIS).

Basilepta pallicornis Chen, 1935, Sinensia 6(3):312 (Tonkin: Lan-Son near Bao-Lac ; PARIS). -Gressitt \& Kimoto, 1961, Pac. Insects Monogr. 1A:228 (Tonkin, W. China). N e w synonymy.

Distribution : Vietnam, W. China.

No additional material.

Basilepta jeanvoinei (Pic) New combination

Nodostoma jeanvoinei Pic, 1928, Bull. Soc.Zool. Fr., 53 : 379 (Tonkin :Chapa; PARIS).

Distribution : Vietnam.

No additional material. 
Basilepta korhongensis Kimoto \& Gressitt, n. sp. (Fig. 7c)

Body oblong oval. General color reddish brown, antenna pitchy black with three or four basal segments brownish; legs entirely reddish brown.

Head with surface smooth, shining, impunctate, clypeus with a pair of sligthly raised short longitudianl costae laterally, vertex with a shallow longitudinal furrow at middle. Antenna robuster, nearly $2 / 3$ as long as body length; first segment robust, somewhat club-shaped; second nearly $3 / 5$ as long as first; third slenderer, nearly as long as second in length; fourth $11 / 3$ times as long as third and gradually widened apically; fifth robuster than fourth and nearly $11 / 4$ times as long as fourth; sixth slightly shorter than fifth and nearly half as wide as long; seventh to ninth subequal to sixth in length and shape; tenth slightly shorter than ninth; eleventh $1 \frac{1 / 2}{2}$ time:s as long as tenth and its apex pointed. Pronotum transverse, about $11 / 2$ times as wide as long, widest almost at middle, lateral margin evenly rounded and not forming a distinct angle, anterior angle with a setigerous puncture, and basal angle slightly tuberculate and with a setigerous puncture; anterior margin slightly rounded anteriorly and posterior margin widely rounded and slightly produced posteriorly at middle; dorsal surface smooth, shining, nearly impunctate, anterior sulcus distinct only on lateral portion and interrupted at middle ; proepimeron smooth, shining, impunctate ; metasternum nearly smooth, shiningim punctate. Scutellum subtrigonate, with apex rouned ; surface slightly convex, smooth, shining, impunctate. Elytron subparallelsided with apex rounded; humerus strongly raised, subbasal area slightly raised but not separated from bethind by distinct transverse furrow; punctures regularly arranged in longitudinal rows, these punctures distinct on basal portion and much finer and become obsolescence on apical portion.

Length 2.6-2.9 $\mathrm{mm}$.

Holotype (Type No. 2350, Kyushu Univ.), THAILAND: Kor Hong, 23. vi. 1965, Y. Miyatake. Paratoptotypes: 2 exs., same data as the' holotype (KU, BISHOP). Paratype : THAILAND: Khao Chong, nr. Trung 1 ex., 25. vi. 1965, K. Morimoto (KIMOTO).

This new species somewhat resembles Basileptabeccarii(Jacoby), but differs in having the anterior sulcus of prothorax interrupted at middle and elytron with the postbasal sulcus obsolescent.

\section{Basilepta lameyi (Lefèvre) New combination}

Nodostoma lameyi Lef., 1893, Ann. Soc. Entomol. Fr. 62: 119 (Tonkin: Ha-Lang; PARIS).

Nodostoma particulare Pic, 1930, Mél. Exot. Entomol. 56: 6 (Tonkin:Chapa; PARIS). Ne w synonymy.

DistRibution : Vietnam.

No additional material. 


\section{Basilepta latipennis (Pic) New combination}

Nodostoma latipenne Pic, 1928, Bull. Soc. 2001. Fr., 53: 379 (Tonkin:Chapa; PARIS).

Distribution : Vietnam.

No additional material.

Basilepta leechi (Jacoby)

Nodosfoma leecki Jac., 1889, Proc.Zool.Soc. Lond.. 1888: 344 (China ; MCZ). Lefèvre, 1839, Ann. Soc. Entomol. Fr. 1893: 119 (Tonkin: Lang-Son, Ha-Lang).

Basilepta leechi: Weise, 1922, Tijdschr. Entomol. 65: 47 (Fokien). -Chen, 1935, Sinensia 6(2): 321 (S. China, Tonkin). -Gressitt \& Kimoto, 1961, Pac. Insects Monogr. 1A: 225 (China).

Distribution : Vietnam, China.

No additional material.

Basilepta livida Kimoto \& Gressitt, n. sp. (Fig. Ila)

Body oval. Bluish black with elytron violaceous blue.

Head with surface smooth, strongly and closely punctate, clypeus with a pair of slightly raised longitudinal short costae laterally. Antenna filiform, nearly $2 / 3$ as long as body length; first segment robust, somewhat club-shaped ; second much slenderer, three to four times as long as wide, and nearly $3 / 4$ as long as first ; third much slenderer than second, and $11 / 5$ times as long as second; fourth subequal to third in length and shape; fifth much robuster than fourth, and $11 / 4$ times as long as fourth ; sixth slightly shorter than fifth ; seventh subequeal to sixth in length and shape; eighth slightly shorter than seventh; ninth and tenth subequal to eighth in length and shape; eleventh $11 / 4$ times as long as tenth and its apex pointed. Pronotum transverse, about, $13 / 4$ times as wide as long, lateral margin with a sharp angle slightly behind middle; anterior angle somewhat denticulate and with a setigerous puncture, and basal angle slightly tuberculate and with a setigerous puncture; anterior margin slightly rounded anteriorly and posterior margin widely rounded and slightly produced posteriorly at middle; dorsal surface distinctly and rather closely punctate, anterior sulcus entire and impressed with a row of disticnt punctures; proepimeron closely and distinctly punctate ; metasternum sparsely covered with fine pubescence and punctures. Scutel. lum subtrigonate, with apex rounded; surface slightly convex, smooth, shining, sparsely impressed by minute punctures, especially on basal portion. Elytron subparallel-sided with apex rounded; humerus strongly raised ; subbasal area slightly convex and separated from behind by a shallow transverse furrow; punctures regularly arranged in longitudianal rows, and these punctures larger and deeper on basal area and finer and shallower on apical area.

Length $4.0 \mathrm{~mm}$. 


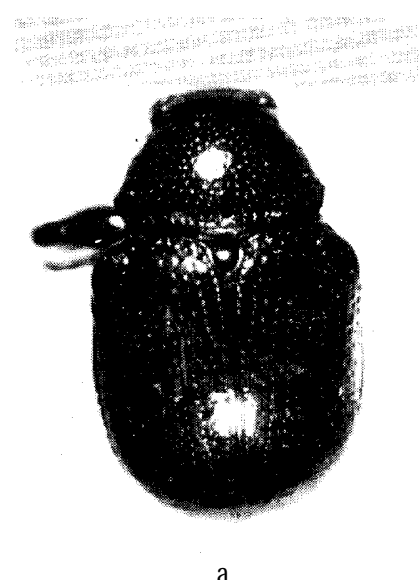

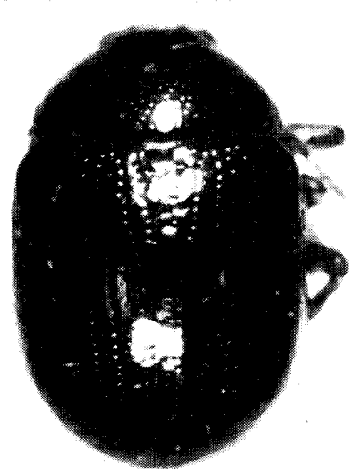

b

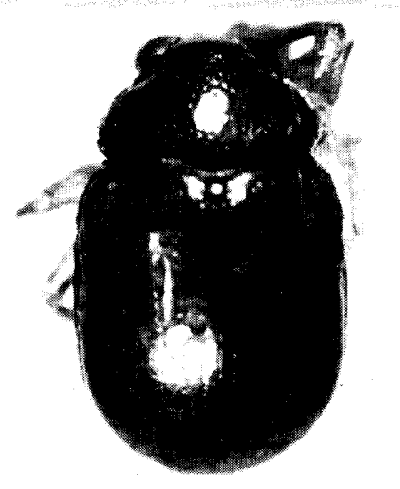

$\mathrm{c}$

Fig. Il. a, Basilepta livida Kimoto \& Gressitt, n. sp.; b, B. airidicyanea Kimoto \& Gressitt, $\mathrm{n}, \mathrm{sp} . ; \mathrm{c}$. B. varicolor (Jacoby).

Holotype (Type No. 2351, Kyushu Univ.), THAILAND : Chiang Mai Prov., Doi Pui, 1685 m, 17. vi. 1965, Y. Miyatake. Paratypes : THAILAND : Chiang Mai Prov., Doi Suthep, 1000 m, 1 ex., 18. vi. 1965, K. Morimoto, 1 ex., 18. viii. 1973, S. Nakao (KU, KIMOTO) ; Doi Suthep, 1300 m, 2 exs., 8. vi. 1965, P. D. Ashlock (BISHOP).

This new species resembles B.leechi (Jacoby) from China, but differs in having pronotum more transverse, widest slightly behind middle and more sharply angulate laterally.

Basilepta longipennis (Pic) New combination

Nodostoma longipenne Pic, 1931, Mél. Exot. Entomol. 57: 23 (Tonkin:Chapa; PARIS).

Distribution : Vietnam.

No additional material.

Basilepta maai Kimoto \& Gressitt, n. sp. (Fig. 9b)

Body oblong oval, convex. General color reddish brown, head with top of vertex blackish, antenna with two or three apical semgents infuscate.

Head distinctly and closely punctate, especially on vertex and slightly less on clypeus. Antenna filiform, nearly $2 / 3$ as long as body length; first segment robust, somewhat club-shaped; second shortest, robust, nearly twice as long as wide and $2 / 3$ as long as first; third slender, nearly $11 / 2$ times as long as second; fourth subequal to third in length and shape; fifth nearly $11 / 4$ times as long as fourth; sixth and seventh subequal to fifth in length and shape ; eighth nearly $3 / 4$ as long as seventh and distinctly robuster; 
ninth and tenth subequal to eighth in length and shape; eleventh nearly $11 / 3$ times as long as tenth and its apex pointed. Pronotum transverse, nearly $13 / 5$ times as wide as long, widest slightly behind middle and strongly narrowed anteriorly and posteriorly, anterior angle somewhat denticulate and with a setigerous puncture, and basal angle slightly tuberculate and with a setigerous puncture ; anterior margin sligthly rounded anteriorly, and posterior margin widely rounded posteriorly ; dorsal' surface closely and distinctly punctate, especially on lateral portion ; anterior sulcus entire and impressed by a row of distinct punctures ; proepimeron closely impressed by distinct punctures basally; metasternum nearly impunctate. Scutellum subtrigonate, with apex rounded; surface slightly convex, smooth, shining, impunctate. Elytron subparallel sided with apex rounded ; subbasal area distinctly raised and separated from behind by a distinct transverse furrow; punctures regularly arranged in longitudinal rows, and these punctures larger and deeper on basal area and much finer and shallower on apical area; humerus strongly raised and with a distinct costa starting from humerus and ending near middle of side.

Length $3.7-4.0 \mathrm{~mm}$.

HоLотуPE (BISHOP), THAILAND : Doi Suthep (water margin), Chiang Mai Prov., 4. iv. 1958, T. C. Maa. Paratopotype: 1 ex., same data as the holotype (KTMOTO). Paratypes : THAILAND: Doi Suthep, 1 ex., 1-5. iv. 1958, 1 ex., 29. iii- 4. iv. 1958, T. C. Maa;Doi Pui, Chang Mai Prov., 1 ex., 2. iv. 1958, T. C. Maa (BISHOP).

Distribution: Thailand.

This new species somewhat resembles B. pallidula Baly, from Far East, but differs in having pronotum widest slightly behind middle, punctures on disc more closely and distinctly punctate, and the top of vertex blackish.

Basilepta marginalis Kimoto \& Gressitt, $\mathbf{n}$ sp. (Fig. 8a)

Body oval; entirely yellowish brown, antenna with apical segments somewhat infuscate.

Head with surface smooth, shining, nearly impunctate except for sparsely impressed punctures on side of vertex. Antenna filiform, nearly $2 / 3$ as long as body length; first segment robust, somewhat club-shaped; second nearly $2 / 3$ as long as first; third slenderer, nearly $11 / 3$ times as long as second; fourth nearly $11 / 4$ times as long as third; fifth subequal to fourth in length but slightly robuster ; sixth subequal to fifth in length but slightly robuster; seventh and eighth subequal to sixth in length and shape; ninth slightly shorter than eighth; tenth subequal to ninth in length and shape; eleventh $11 / 3$ times as long as tenth and its apex pointed. Pronotum transverse, nearly twice as wide as long, widest slightly before base and forming subbasal angle which is not sharp but rounded, and widely collared at near 
basal angle, and side abruptly converged anteriorly in almost straight line ; anterior angle with a setigerous puncture and basal angle slightly tuberculate and with a setigerous puncture; anterior margin slightly rounded anteriorly and posterior margin widely rounded and slightly produced posteriorly at middle; dorsal surface smooth, shining, nearly impunctate, anterior sulcus entire and impressed by a row of very fine punctures; proepimeron with surface finely wrinkled, nearly impunctate ; metasternum sparsely covered with fine hairs and minute punctures. Elytron subparallel-sided with apex rounded; humerus raised; subbasal area slightly covnex, and separated from behind by a shallow transverse furrow; punctures arranged in longitudinal rows, and these punctures slightly finer on subbasal area, and much finer and become obsolescence on apical area.

Length 2.6- $2.7 \mathrm{~mm}$.

Holotype (Type No. 2352, Kyushu Univ.), THAILAND: Chiang Mai Prov., Chiang Dao, 15. vi. 1965, K. Morimoto. Paratopotypes: 7 exs., same data as the holotype (KU, KIMOTO). Paratypes : THAILAND: Mae Talai, nr. Chieng Dao, 1 ex., 3. vii. 1970, upland rice, K. Yano (KU) ; Chieng Dao, 1 ex., 15. vi. 1965, P. D. Ashlock (BISHOP).

This new species somewhat resembles B.davidi(Lefèver), but differs in having clypeus nearly impunctate and the lateral margin widely collared.

\section{Basilepta martini (Lefèvre)}

Nodostoma martini Lef., 1885, Bull. Soc. Entomol. Fr., ser. 6, 5: 65 (Tonkin); 1889, ibid. ser. 6, 9: 296(Tonkin: Lien-Son) ; 1893, ibid. 1893: 117 (Tonkin, Cambodge : Luang-Prabang). -Jacoby, 1896, Ann. Mus. Civ. Genova 37: 129 (Sumatra).

Nodostoma martini var. atrovittata Pic, 1930, Bull. Soc. Linn. Lyon 9: 37 (Tonkin).

Basilepta martini: Chen, 1935, Sinensia 6(2): 308 (S. China, Tonkin, Cochinchina, Combodge). --Gressitt \& Kimoto, 1961, Pac. Insects Monogr. 1A: 226 (China, Hainan).

Basilepta sauteri Chit jô, 1938, Arb. Morph. Taxon. Entomol. Berlin-Dahlem 5(1): 26 (Formosa ; DEI) ; 1956, Philip. J. Sci. 85(1): 31 (Formosa). --Gressitt\& Kimoto, 1961, Pac. Insects Monogr. 1A: 226 (= martini).

Distribution : Thailand, Laos, Cambodia, Vietnam, S. China, Hainan, Taiwan.

Material ExAmined. THAILAND: Kaochong, Trang, 1 ex., 9. iv. 1960 (BANGKHEN). LAOS: Umgeb. Vientiane, 3 exs., iii-iv. 1963 (MUNCHEN) ; Ban Van Heue, $20 \mathrm{~km}$ E of Phou-kow-kuei, 1 ex., 15. vi. 1966, Native collr ; Vientiane Prov., Vientiane, 1 ex., 22. vii. 1965, Native collr (BISHOP). CAMBODIA: Damrey Phong, 1 ex., 14-16. iv. 1961, N. R. Spencer (BISHOP). VIETNAM: Hoa-Binh, Tonkin, 2 exs., A Cooman (FREY).

Basilepta melanopus (Lefèvre)

Nodostoma melanopus Lef., 1893, Ann. Soc. Entomol. Fr. 62: 119 (Tonkin ; PARIS).

Basilepta melanopus: Chen, 1935, Sinensia 6(2): 316 (Tonkin).--Gressitt \& Kimoto, 1961, 
Pac. Insects Monogr. 1A: 226 (Tonkin, S. China).

Distribution : S. China, Vietnam.

No additional material.

Basilepta minutissima Kimoto \& Gressitt, n. sp. (Fig. 9a)

Body oval, slightly convex. General color pitchy brown, antenna almost entirely yellowish brown, legs reddish brown with femora pitchy black.

Head with surface smooth, strongly but not so closely punctate, clypeus with a pair of slightly raised longitudinal short costae laterally, vertex with a short longitudinal sulcus at middle. Antenna robuster; nearly $2 / 3$ as long as body length; first segment robust, somewhat club-shaped ; second nearly $3 / 4$ as long as first, robust; third slightly shorter than second and much slenderer; fourth subequal to third in length and shape; fifth slightly longer than fourth and much robuster; sixth to eighth subequal to fifth in length and shape ; ninth subequal to eighth in length but slightly robuster; tenth subequal to ninth in length and shape; eleventh $13 / 5$ times as long as tenth and its apex pointed. Pronotum transverse, nearly $13 / 4$ times as wide as long, widest almost at middle and strongly narrowed anteriorly and posteriorly, anterior angle with a setigerous puncture, and basal angle slightly tuberculate and with a setigerous puncture; anterior margin sligthly rounded anteriorly, and posterior margin rounded and slightly produced posteriorly at middle ; dorsal surface smooth, shining, distinctly and homogeneously impressed by large punctures which are much larger than average elytral punctures and separated by approximately their diameters, anterior sulcus distinct only on lateral portion and interrupted at middle; proepimeron smooth, shining, distinctly impressed by large punctures ; metasternum smooth, shining, nearly impunctate. Scutellum subtrigonate, with apex rounded; surface slightly convex, smooth, shining, impunctate. Elytron subparallel sided with apex rounded; humerus distinctly raised, subbasal area slightly raised, subbasal area slightly convex and separated from behind by a shallow transverse furrow ; punctures regularly arranged in longitudinal rows, and these punctures become finer in apical portion, and with a slightly raised short costa starting from humerus.

Length 1.8-1. $9 \mathrm{~mm}$.

Holotype (Type No. 2358, Kyushu Univ.), THAILAND: Mae Klang Water Fall, nr. Chom Thong, 11. vi. 1965, K. Morimoto. Paratopotype: 1 ex., same data as the holotype (BISHOP).

This new species somewhat resembles B.incerta(Pic), but differs in being body length shorter and having pronotum more elongate and more thickly covered with large punctures. 
Basilepta miyatakei Kimoto \& Gressitt, n. sp. (Fig. 8b)

Body oval. Dark reddish brown, elytron black with a large median marking yellowish brown, antenna pitchy black with two or three basal segments brownish, legs pitchy brown to black.

Head with surface smooth, distinctly impressed by large punctures, vertex convex. Antenna relatively robuster, especially on median segments, slightly depressed, nearly $2 / 3$ as long as body length: first segment robust, somewhat club-shaped ; second shortest, nearly $4 / 5$ as long as first; third $11 / 4$ times as long as second, slenderer; fourth about $11 / 5$ times as long as third, gradually widened apically; fifth robuster, nearly three times as long as wide, slightly shorter than fourth; sixth and seventh subequal to fifth in length and shape; eighth slightly shorter than seventh; ninth and tenth subequal to eighth in length and shape; eleventh longest, nearly $11 / 3$ times as long as tenth and its apex pointed. Pronotum transverse, about $11 / 2$ times as wide as long, lateral margin with a sharp angle slightly behind middle; anterior angle somewhat denticulate and with a setigerous puncture, and basal angle slightly tuberculate and with a setigerous puncture; anterior margin slightly rounded anteriorly and posterior margin widely rounded and slightly produced posteriorly at middle; dorsal surface distinctly but not closely punctate, anterior sulcus entire and impressed with a row of distinct punctures; proepimeron closely and distinctly punctate ; metasternum sparsely impressed by fine punctures. Scutellum subtrigonate, with apex rounded ; surface slightly convex, smooth, shining, impunctate. Elytron subparallelsided with apex rounded; humerus strongly raised; subbasal area slightly convex and separated from behind by a shallow transverse furrow; punctures regularly arranged in longitudinal rows, and these punctures larger and deeper on basal area and much finer and shallower on apical area.

Length $3.8-4.8 \mathrm{~mm}$.

Holotype (Type No. 2354, Kyushu Univ.), THAILAND: Khao Chong, nr. Trung, 26. vi. 1965, Y. Miyatake. Paratopotypes: 4 exs., same data as the holotype (KU, BISHOP, KIMOTO). Paratype : 1 ex., same data as the holotype but $\mathrm{K}$. Morimoto (KU).

This new species somewhat resembles B. fulvofasciata Jacoby, from India, in having elytron black with fulvous band, but differs in being the body length larger and having pronotum more longer and the elytral marking free from sutural margin.

Basilepta morimotoi Kimoto \& Gressitt, n. sp. (Fig. 10b)

Body oblong oval, convex. Head pitchy black with middle of vertex brownish, prothorax blackish with anterior margin slightly brownish ; scutellum and elytron reddish brown, antenna pitchy black with basal segments brown- 
ish; legs and ventral surfaces of thorax pitchy black and abdomen dark reddish brown.

Head with surface smooth, strongly and rather closely punctate, vertex convex. Antenna robuster, nearly $2 / 3$ as long as body length; first segment robust, somewhat club-shaped; second much slender, nearly three times as long as wide, and nearly $4 / 5$ as long as first; third nearly as long as second, but much slenderer ; fourth nearly $11 / 2$ times as long as third; fifth subequal to fourth in length, but much robuster and thickly covered with fine pubescence; sixth slightly shorter than fifth and nearly twice as long as wide ; seventh subequal to sixth in length and shape; eighth slightly shorter than seventh; ninth and tenth subequal to eighth in length and shape; eleventh slightly longer than tenth and its apex pointed. Pronotum transverse, about $13 / 5$ times as wide as long, lateral margin with a sharp angle slightly behind middle; anterior angle somewhat denticulate and with a setigerous puncture, and basal angle sligthly tuberculate and with a setigerous puncture; anterior margin sligthly rounded anteriorly and posterior margin widely rounded and slightly produced posteriorly at middle ; dorsal surface distinctly but not closely punctate, anterior sulcus distinct only on lateral portion and interrupted at middle; proepimeron rather closely impressed by large punctures; episternum rather closely impressed by large punctures. Scutellum subtrigonate, with apex rounded; surface slightly convex, smooth, shining, nearly impunctate. Elytron subparallel-sided with apex rounded ; humerus strongly raised; subbasal area distinctly raised and separated from behind by a deep transverse furrow; punctures regularly arranged in longitudinal rows, and these punctures larger and deeper but slightly finer on subbasal area, and much finer and become obsolescent on apical area.

Length 3. $4 \mathrm{~mm}$.

нодотуре (Type No. 2355, Kyushu Univ.), THAILAND: Chiang Mai Prov., Doi Pui, $1300 \mathrm{~m}$, 17. vi. 1965, K. Morimoto. Paratopotype: 1 ex., same data as the holotype (BISHOP).

This new species somewhat resembles B.semirufa(Pic), but differs in having prothorax with a sharp angle slightly behind middle, and prothorax blackish and elytron reddish brown.

Basilepta multimaculata Kimoto \& Gressitt, n. sp. (Fig. 13b)

Body oblong oval; general color dark yellowish brown, pronotum with a pair of large median markings pitchy black, elytron with basal, laterobasal, postmedian and latero-median markings pitchy black; antenna reddish brown with apical segments slightly infuscate; ventral surfaces mostly pitchy black with apical segments of abdomen partly brownish; legs reddish brown with apex of femora and base of tibiae blackish.

Head with surface smooth, strongly and rather closely punctate. Antenna 
filiform, nearly $2 / 3$ as long as body length; first segment robust, somewhat club-shaped ; second nearly $2 / 3$ as long as first, and nearly three times as long as wide; third nearly $11 / 2$ times as long as second and much slenderer; fourth nearly $11 / 3$ times as long as third; fifth to seventh subequal to fourth in length and shape; eighth slightly shorter than seventh, ninth subequal to eighth in length and shape; tenth slightly shorter than ninth; eleventh nearly $11 / 3$ times as long as tenth and its apex pointed. Pronotum transverse, about $14 / 5$ times as wide as long, widest slightly before basal corner and strongly narrowed anteriorly ; anterior margin slightly rounded anteriorly and posterior margin rounded and slightly produced at middle; anterior angle somewhat denticulate and with a setigerous puncture, and basal angle slightly tuberculate and with a setigerous puncture; surface distinctly and closely punctate, and punctures larger than, or subequal to average elytral punctures, and anterior sulcus deep, entire and impressed by a row of distinct punctures; lateral margin widely collared apically, and width of collar much wider than width of apical segments of antenna; proepimeron finely granulate and its basal portion rather closely impressed by distinct punctures; metasternum slightly wrinkled, sparsely covered with fine hairs and minute punctures. Scutellum subtriangular, with apex rounded ; surface finely granulate, distinctly impressed by fine punctures especially on basal portion. Elytron subparalle1sided with apex rounded; subbasal area slightly convex and separated from behind by a shallow but distinct transverse furrow; punctures regularly arranged in longitudinal rows and these punctures slightly finer in apical portion, interstices of these puncture rows slightly raised in basal and lateral portions; humerus distinctly raised. and with a short but distinct costa starting from humerus.

Length $4.5-4.8 \mathrm{~mm}$.

HoLotype (BISHOP), VIETNAM: $20 \mathrm{~km}$ SE of Pleiku, $400 \mathrm{~m}, \mathbf{1 2}$. v. 1960, L. W. Quate. Paratopotypes : 2 exs., same data as the holotype (BISHOP, KIMOTO).

This new species somewhat resembles B.chiangmaiensis Kimoto \& Gressitt, but differs in having the punctures of pronotum more closely and distinctly impressed and lateral margin of pronotum more widely collared.

Basilepta notabilis Chen

Basilepta notabile Chen, 1935. Sinensia 6(3): 310 (Laos or Cambodia ; PARIS). -Gressitt \& Kimoto, 1961, Pac. Insects Monogr. 1A: 227 (Laos or Cambodia).

Distribution : Laos or Cambodia.

No additional material.

Basilepta puncticollis (Lefèvre) (Fig. 12c)

Nodostoma puncticolle Lef., 1889, Ann. Soc. Entomol. Fr., ser. 6, 9: 295 (Tonkin); 1893, ibid. 62 : 116 (Tonkin, Langson). --Jacoby, 1908, Fauna India, Coleopt. 2: 321 (India, 


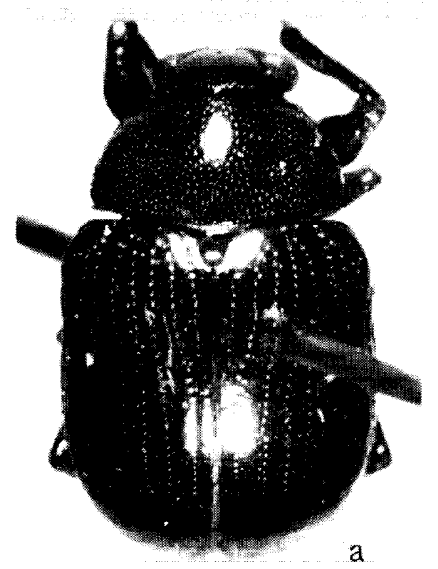

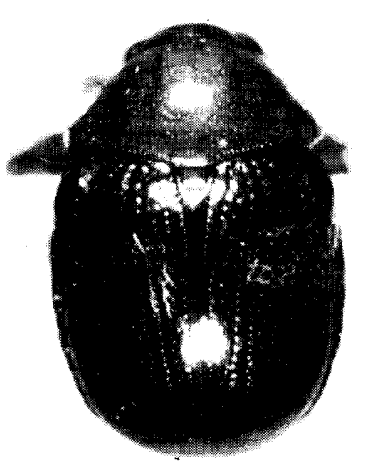

b

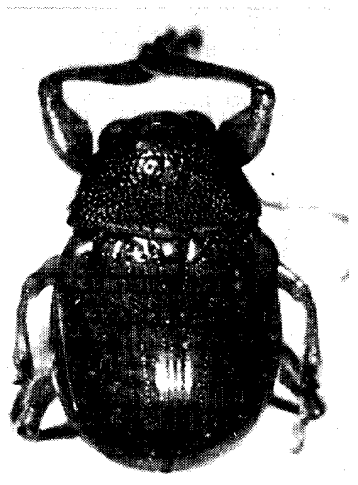

$\mathrm{c}$

Fig. 12. a, Basilepta rondoni Kimoto \& Gressitt, n. sp. ; b, B. frontalis (Baly); c, B. puncticollis (Lef èvre).

Burma, Tonkin).

Nodostoma birmanicum Jacohy, 1892, Ann. Mus. Civ. Geneva 32: 898 (Burma ; GENOVA) ; 1908, Fauna India, Coleopt. 2:321 (=puncticolle).

Nodostoma Zimbatum Lefevre, 1893, Ann. Soc. Entomol. Fr. 1893 :116 (Cochinchina ; PARIS). $\mathrm{N}$ ew synonymy.

Nodostoma nigriventre Lefevre, 1893, Ann. Soc. Entomol. Fr. 1893: 117 (Cochinchina; PARIS). New synonymy.

Basilepta robusta Weise, 1922, Tijdschr. Entomol. 65: 48 (China : Fukien ; STOCKHOLM). Chen, 1935, Sinensia 6(3): 313 (Fokien). Gressitt\& Kimoto, 1961, Pac. Insects Monogr. 1A: 230 (S. China). New synonymy.

Nodostoma atrithorax Pic, 1930, Mél. Exot. Entomol. 56: 5 (Yunnan ; with var. notaticeps.p.6; PARIS). -Gressitt \& Kimoto, 1961, Pac. Insects Monogr. 1A:230 (=robusta).

Nodostoma atritarse Pic, 1930, Mél. Exot. Entomol. 56: 7 (China ; PARIS). -Gressitt \& Kimoto, 1961, Pac. Insects Monogr. 1A: 230 (=robusta).

Nodostoma brunneopunctatum Pic, 1933, Mél. Exot. Entomol. 61: 8 (China : Fukien ; PARIS). Gressitt \& Kimoto, 1961, Pac. Insects Monogr. 1A:230 (=robusta).

Nodostoma femorale Pic, 1934, Mél. Exot. Entomol. 64: 7 (Tonkin; with var. hanoiense; PARIS). New synonymy.

Basilepta puncticolle: Chen, 1935, Sinensia 6(3):314 (Tonkin, Burma, Punjab). Gressitt \& Kimoto, 1961, Pac. Insects Monogr. 1A: 229 (China). Kimoto\& Takizawa, 1973, Kontyû 41(2): 175 (Nepal).

Basilepta atrithorax: Chen, 1935, Sinensia 6(3): 309 (Yunnan).

Basilepta atritarse: Chen, 1935, ibid. (China).

Basilepta bruneopunctata: Chen, 1940, ibid. 11(5-6): 502 (China).

Distriburron: India, Burma, Nepal, Thailand, Laos, Vietnam, S. China.

We could not trace the type location of puncticolle Lefevre. Our identification is based on the specimen identified by Lefevre as "Nodostoma punctithorax Lef. (sic ?)". This specimen is now preserved in Paris Museum.

Material examined. THAilAND: Kanchanaburi, 3 exs., 31. v. 1962 (BANGKHEN) ; Chiangmai Prov., Doi Suthep, 3 exs., 18-19. vi. 1965, K. Morimoto \& Y. 
Miyatake; Fang, 9 exs., 13-14. vi. 1965, K. Morimoto; Chieng Dao, 4 exs., 15. vi. 1965, K. Morimoto \& Y. Miyatake (KU). LAOS: Ban Van Heue, $20 \mathrm{~km} \mathrm{E}$ of Phou-kow-kuei, 1 ex., 15-31. v. 1965, Native collr (BISHOP) ; Umgeb. Pakse, 1 ex., 1963 (MUNCHEN). VIETNAM: Hoa-Binh, Tonkin, 4 exs., A. Cooman (FREY)?

\section{Basilepta quadrimaculata (Pic) New combination}

N odostoma quadrimaculatum Pic. 1931, Bull. Soc. Linn. Lyon 10:27 (Tonkin:Chapa; PARIS).

Distribution : Vietnam.

No additional material.

Basilepta rondoni Kimoto \& Gressitt, n. sp. (Fig. 12a)

Body oval; dorsum reddish cupreous, with greenish luster especially on lateral part; antenna reddish brown; ventral surfaces dark reddish brown with thorax dark to blackish brown; legs reddish brown.

Head strongly and rather closely punctate, interstices of punctures smooth, shining; vertex with a short longitudinal furrow in middle. Antenna filiform, nearly $2 / 3$ as long as body length; first segment robust, somewhat clubshaped; second nearly $2 / 3$ as long as first, robust; third nearly as long as second in length but much slenderer; fourth nearly $11 / 5$ times as long as third; fifth subequal to fourth in length but much robuster; sixth slightly shorter than fifth and nearly three times as long as wide; seventh to ninth subequal to sixth in length and shape; tenth slightly shorter than ninth; eleventh nearly $12 / 5$ times as long as tenth and its apex pointed. Pronotum transverse, about $14 / 5$ times as wide as long, rounded at side, not forming angle and widest slightly before basal corner ; anterior margin slightly rounded anteriorly and posterior margin rounded and slightly produced posteriorly; anterior and posterior corners each with a setigerous puncture; dorsal surface closely impressed by distinct punctures and more closely so laterally, interstices of punctures nearly as wide as diameter of punctures, anterior sulcus distinct only on lateral portion and interrupted at middle; proepimeron finely granulate and closely impressed by large punctures especially on basal portion ; metasternum wrinkled and sparsely impressed by shallow but fairly large punctures. Scutellum elongate, subquadrate and rounded at apex; convex, smooth, sparsely covered with fine pubescence basally. Elytron subparallel-sided, with apex rounded; humerus distinctly raised, subbasal area slightly raised, and subbasal transverse furrow obsolescent and hardly separated from behind; punctures regularly arranged in longitudinal rows, and these punctures become finer in apical portion.

Length 4. 6-6.7 $\mathrm{mm}$.

Holotype (BISHOP), LAOS: Muong Sing, NW of Luang Prabang, 650 m, 6-10. vi. 1960, S. Quate \& L. W. Quate. Paratypes: LAOS: Ban Van Eue, 1 ex., 20 


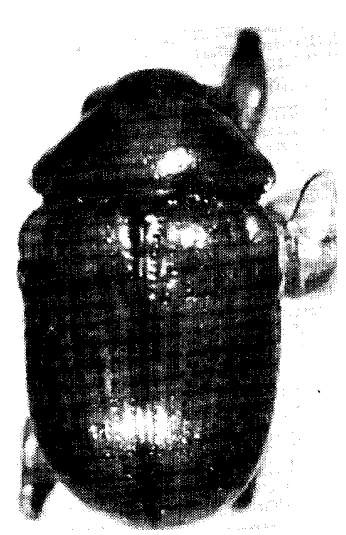

a

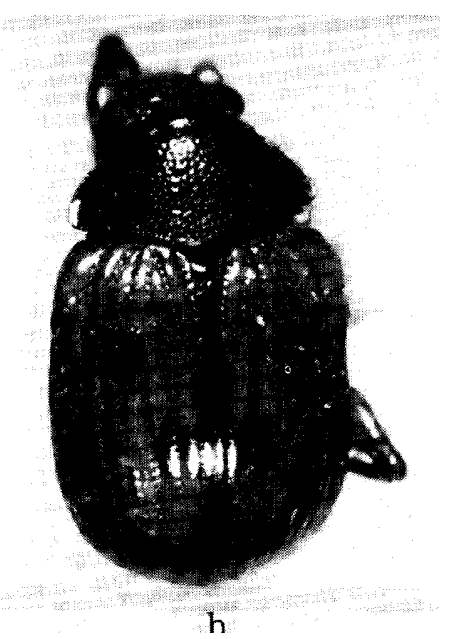

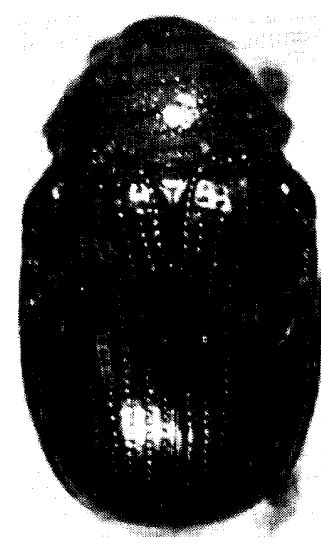

c

Fig. 13. a, Basilepta chiangmaiense Kimoto \& Gressitt, n. sp. ; b, B. multimaculatum Kimotu \& Gressitt, n. sp. ; c. B. subcostatum (Jacoby).

km E of Phou-kow-kuei, 1 ex., 15-31. v. 1965, Native collr (BISHOP) ; Vientiane Prov., Vientiane, 1 ex., 22. vii. 1965, Native collr (KIMOTO).

Judging from the original description, this new species seems to resemble Basilepta speciosa (Lefèvre) from Cochinchina, but differs in having the punctures of pronotum much stronger and closer.

\section{Basilepta semirufa(Pic) New combination}

Nodostoma semirufumPic, 1930, Mél. Exot. Entomol. 56: 6 (Tonkin: Chapa; PARIS).

Distribution: Vietnam.

No additional material.

Basilepta speciosa (Lefèvre) N ew combination

Nodostoma speciosum Lef., 1893, Ann. Soc. Entomol. Fr. 62: 120 (Cochinchina).

Distribution: vietnam.

We could not trace the type location of this species. No additional material.

Basilepta spenceri Kimoto \& Gressitt, n. sp. (Fig. 7b)

Body oval; general color reddish brown, antenna pitchy black with three or four basal segments brownish, legs reddish brown apical half of femora and basal portion of tibiae pitchy black.

Head with surface smooth, shining, impunctate, clypeus with a pair of longitudinal short costae laterally and separated from vertex by a shallow transverse depression, vertex with a short longitudinal sulcus at middle. 
Antenna relatively robust, nearly $2 / 3$ as long as body length and preapical segments nearly $21 / 2$ times as long as wide ; first segment robust, somewhat club-shaped ; second nearly $3 / 4$ as long as first and nearly $21 / 2$ times as long as wide ; third slenderer, and slightly longer than second; fourth slightly longer than third; fifth subequal to fourth in length but much robuster ; sixth subequal to fifth in length but slightly robuster; seventh and eighth subequal to sixth in length and shape; ninth slightly shorter than eighth; tenth sub. equal to ninth in length and shape; eleventh $11 / 4$ times as long as tenth and its apex pointed. Pronotum transverse, about $12 / 3$ times as wide as long, side evenly rounded, widest almost at middle and narrowed anteriorly and posteriorly ; anterior margin distinctly produced anteriorly and posterior margin slightly rounded and produced posteriorly at middle; anterior and posterior corners each with a setigerous puncture and posterior corner slightly tuberculate ; dorsal surface nearly impunctate, smooth, shining, anterior sulcus distinct only on lateral portion and interrupted at middle; proepimeron smooth, nearly impunctate ; metasternumm smooth, shining, nearly impunctate. Scutellum subtriangular, with apex rounded; surface smooth, shining, slightly convex, impunctate. Elytron subparallel-sided with apex rounded; humerus distinctly raised ; subbasal area distinctly convex, subbasal transverse impression obsolescent; punctures regularly arranged in longitudinal rows and these punctures distinct in basal portion and become obsolescent in apical portion.

Length $2.4-2.7 \mathrm{~mm}$.

Holotype (BISHOP), THAILAND: Fyan, 900-1000 m, 11. vii. -9. vii. 1961, N. R. Spencer. Paratopotypes: 2 exs., same data as the holotype (BISHOP, KIMOTO) .

This new species resembles B.varicolor Jacoby, in having the side of prothorax evenly rounded, but differs in having pronotum nearly impunctate and widest almost at middle.

Basilepta subcostata (Jacoby) (Fig. 13c)

Nodostoma subcostatum Jac., 1889, Ann. Mus. Civ. Genova 27: 164 (Burma ; GENOVA. BM); 1908, Fauna India, Coleopt. 2: 334 (Assam, Burma).

Nodostoma cyanipenne Lefèvre, 1893, Ann. Soc. Entomol. Fr. 62: 120 (Tonkin ; PARIS). New synonymy.

Basilepta subcostatum: Chûjô, 1964, Nature and Life in SE Asia, Kyoto 3: 268 (Thailand: Phattalung).

Distribution : India, Burma, Thailand, Laos.

Material exAmined. THAILAND: Banna, Chawang, nr Nabon, 70 m, 1 ex., 6. ix. 1958, J. L. Gressitt (BISHOP) ; Fang, 1 ex., 13. vi. 1965, Y. Miyatake (KU) ; Rayong, 2 exs., 17. vii. 1965 (BANGKHEN). LAOS: Umgeb. Vientiane, 1 ex., iii-vi. 1963 (MUNCHEN) ; Ban Van Heue, 20 km E of Phou-kow-kuei, 4 exs., 115. v. 1965, J. A. Rondon (BISHOP). 


\section{Basilepta tricolor (Baly) New combination}

N odostoma tricolor Baly, 1877, Trans. Entomol. Soc. Lond., 1877: 38 (Siam; with var. pachy bouri; BM).

N odostoma harmandi Lef., 1893, Ann. Soc. Entomol. Fr. 62: 117 (Cochinchina; PARIS). New synonymy.

N odostoma laosense Pic, 1930, Mél. Exot. Entomol. 56: 5 (Laos ; PARIS). New synonymy.

Basilepta pretiosum:Chûjô, 1964, Nature and Life in Asia, Kyoto 3: 268 (Thailand :Doi Suthep, Chiengmai).

Distribution : Thailand, Laos, Vietnam.

Material examined. THAiland: Chiang Mai Prov., Doi Suthep, 1300 m, 1 ex., 8. vi. 1965, P. D. Ashlock; Chiang Mai (Zoo), 1 ex., 16. vi. 1965, P. D. Ashlock (BISHOP) ;Doi Suthep, $1000 \mathrm{~m}, 4$ exs., 8. vi. 1965, A. Asahina \& K. Morimoto, 8 exs., 12. vi. 1965, 3 exs., 17-19. vi. 1965, K. Morimoto \&. Y. Miyatake ; Tankeo, Doi Suthep, 800 m, 2 exs., 10. vi. 1965, Y. Miyatake ; Fang, 3 exs., 14. vi. 1965, K. Morimoto; Chieng Dao, 2 exs., 15. vi. 1965, K. Morimoto; Mae Sa Water Fall, 1 ex., 10. vi. 1965, 2 exs., 16. vi. 1965, Y. Miyatake (KU). LAOS: Muong Sing, NW of Luang Prabang, $650 \mathrm{~m}, 1$ ex., 6-10. vi. 1960, S. Quate \& L. W. Quate; Sayaboury Prov., Sayaboury, 1 ex., 17. v. 1965, Native collr (BISHOP).

\section{Basilepta varicolor (Jacoby) (Fig. llc)}

N odostoma varicolor Jac., 1885, Proc. Zoo!. Soc. Lond., 1885: 751 (Japan ; BM).

Pagria viridicollis Pic, 1928, Mel. Exot. Entomol. 53: 34 (Tonkin:Hoa-Binh; PARIS). New synonymy.

N odostoma coomani Pic, 1931, Mel. Exot. Entomol. 57: 23 (Tonkin: Hoa-Binh; PARIS). New synonymy.

Basilepta maebarai Chûjô, 1956, Mem. Fac. Lib. Arts \&Educ. Kagawa Univ. 2(31): 1, fig. 1 (Japan; CHUJO). Chûjô \& Kimoto, 1961. Pac. Insects 3(1): 140 (=varicolor).

Basilepta aeneomicans Chûjô, 1956, Philip. J. Sci. 85(1): 26 (Formosa ;TARI). New synonymy.

Basilepta varicolor : Gressitt \& Kimoto, 1961, Pac. Insects Monogr. 1A: 231 (S. China, Hainan). -Kimoto, 1964, J. Fac. Agr. Kyushu Univ. 13(2): 248 (Japan).

Distriburton: Thailand, Vietnam, S. China, Hainan, Taiwan, S. Japan.

Material examined. THAiland : Chiangmai Prov., Doi Suthep (water margin), 1 ex., 4. iv. 1958, T. C. Maa; Doi Suthep, 900 m, 1 ex., 14. xi. 1957, J. L. Gressitt (BISHOP) ; Doi Suthep, 1000 m, 1 ex., 12. vi. 1965, Y. Miyatake (KU). VIETNAM: Hoa-Binh, Tonkin, 1 ex., A. Cooman (FREY).

\section{Basilepta viridicyanea Kimoto \& Gressitt, n. sp. (Fig. 11b)}

Body oval ; dorsal surfaces greenish blue; ventral surfaces black; antenna pitchy black with four or five basal segments brownish; legs entirely pitchy black.

Head with surface strongly and rather closely punctate, vertex with a short shallow longitudinal furrow at middle ; interspace of eyes narrower. Antenna robuster, distinctly longer than half as long as body length; first 
segment robust, somewhat club-shaped; second robust, nearly $2 / 3$ as long as first ; third slender, slightly shorter than second ; fourth slender, $1 \frac{1 / 3}{3}$ times as long as third; fifth subequal to fourth in length and shape; sixth subequal to fifth in length but gradually widened apically; seventh subequal to sixth in length and shape; eighth nearly as long as seventh but much robuster, and nearly twice as long as wide; ninth and tenth subequal to eighth in length and shape; eleventh $11 / 3$ times as long as tenth and its apex pointed. Pronotum transverse, about twice as wide as long, side rounded, not forming angle, widest slightly before basal margin and strongly narrowed anteriorly; anterior and posterior corners somewhat thickened and each with a setigerous puncture; anterior margin slightly rounded anteriorly and posterior margin rounded and distinctly produced posteriorly at middle; anterior sulcus distinct on lateral portion and shallower at middle and impressed by a row of distinct punctures ; dorsal surface closely and distinctly punctate and their interstices closely impressed by fine punctures; pro-epimeron closely and distinctly punctate ; metasternum wrinkled, sparsely impressed by large punctures. Scutellum subtrigonate, with apex rounded; surface finely granulate at middle, impunctate. Elytron rounded at side, widest slightly behind humerus and gradually narrowed posteriorly and apex rounded; subbasal area slightly convex, subbasal transverse furrow obsolete at middle and slightly impressed laterally; punctures regularly arranged in longitudinal rows, and punctures larger and deeper on basal area and finer and shallower on apical area, and their interstices distinctly impressed by fine punctures; humerus distinctly raised.

Length $2.7 \mathrm{~mm}$.

Holotype (BISHOP), VIETNAM: $50 \mathrm{~km} \mathrm{SW}$ of Pleiku, $250 \mathrm{~m}, 14 . \mathrm{v}$. 1960, L. W. Quate. Paratype: VIETNAM: Ban Me Thuot, 500 m, 1 ex., 16-18. v.1960, L. W. Quate (KIMOTO).

This new species resembles Basileptavaricolor(Jacoby), but differs in having the anterior sulcus of pronotum entire and antenna more robuster.

Basilepta viridis (Pic) New combination (Fig. 10c)

Mimoparascela viridis Pic, 1935, Mel. Exot. Entomol. 65: 2(Tonkin; PARIS).

Distribution: Vietnam.

No additional material.

\section{Genus Scelodonta Westwood}

Scelodonta Westw., 1837, Proc.Zool.Soc. Lond., 5: 129 (type : Scelodonta curculionoides Westw., Philippines). -Baly, 1867. Trans. Entomol. Soc. Lond., ser. 2, 4(2): 155. -Chapuis, 1874, Genera Coleopt. 10: 266. -Lefèvre, 1877, Ann. Soc. Entomol. Fr., ser. 5, 7: 159; 1885, Mém. Soc. Sci. Liege, ser. 2, 11: 67. -Jacoby, 1908, Fauna India, Coleopt. 2: 382. -Chen, 1935, Sinensia 6(3):327; 1940, ibid. 11(5-6): 489. -Chûjô, 1956, 
Philip. J. Sci. 85(1): 76. -Gressitt \& Kimoto, 1961, Pac. Insects Monogr. 1A:242.Kimoto, 1964, J. Fac. Agr. Kyushu Univ. 13(2): 251.

Heteraspis Chevrolat, 1837 (nec Leconte, 1859), in Dejean, Cat. Coloept. ed. 3: 437; 1849, d'Orbingny, Dict. Univ. d'Hist. Nat., 13 (type : Eumolpus vittatus Olivier). -Jacoby, 1898, Ann. Soc. Entomol. Belg., 42: 186. --Monrós\& Bechyné, 1956, Entomol. Arb. Mus. Frey $7(3): 1126$.

Odontionopa Motschulsky, 1866 (nec Erichson, 1842, nec Chevrolat, 1837), Bull. Soc. Hist. Nat. Moscou, 2, 29: 408.

Scelodontomorpha Pic, 1938, Mé1. Exot. Entomol. 70: 26 (type : Scelodontomorpha tricostata Pic, Congo Fr.).

\section{Key to SPECIES of Scelodonta}

1(0). Elytron with quite long white hairs

Elytron with very small short hairs only

2(1). Elytoron with four longitudinal stripes of pale golden pubescence ; metallic cupreous, antenna with apical segments black; length 6.0-7.7

cture-rows pubescence, and a spot im-

vittata

Elytron with interstices of puncture-rows pubescence, and a spot immediately below base close to suture, an elongate spot at shoulder, and a third at middle below basal spot, purplish cupreous; greenish to metallic cupreous, terminal segments of antenna and tarsi black; length 3. 2-3. $8 \mathrm{~mm}$

3 (1). Elytron closely punctured behind middle, where there are about 14 puncture-rows ; elytral disc with sharp ridges at base and side; metallic cupreous, antenna with apical segments black; length 3.8-4.0 mmtron sparsely punctured behind middle, where there are about 10 puncture-rows ; elytral disc with low ridges at base and side; coloration of dorsal surfaces variable, mostly aeneous and in some cases bluish, greenish; antenna with apical segments black; length 3.2-4.0 mm …. lewisii

\section{Scelodonta dillwyni (Stephens) (Fig. 14c)}

Eumolpus dillwyni Steph., 1831, Ill. Brit. Entomol. 4: 364, pl. 23, fig. 3 (England: S. Wales). Scelodonta dillwyni: Champion, 1899, Entomol. Month. Mag., ser. 2, 10: 269 (England,Borneo, Singapore, Sumatra, Penang, Siam, India, China). Jacoby, 1908, Fauna India, Coleopt. 2: 385 (Tenasserim, Borneo, Singapore). -Weise, 1922, Philip. J. Sci. 21(5): 451 (Philippines). -Gressitt \& Kimoto, 1961, Pac. Insects Monogr. 1A: 243 (China, Hainan, Vietnam, Thailand). -Kimoto\& Takizawa, 1973, Kontyù, Tokyo 41(2): 176 (Nepal).

Scelodonta nitidula Baly, 1862, Descr. n. gen. \&spec. Phytoph., 2 (Borneo, Singapore) ; 1867, Trans. Entomol. Soc. London, ser. 3, 4(1): 157 (Borneo, Singapore). -Lefèvre, 1889, Ann. Soc. Entomol, Fr. 1889: 291 (Cochinchina : Saigon ; Annam: Hue ;Cambodge : Pnomh-Penh) ; 1893, ibid. 1893 : 121 (Env. Bangkok). -Champion, 1899, Entomol. Month. Mag., ser. 2, 10: 269 (=dillwyni). 一Weise, 1913, Philip. J. Sci. 8 (3)D: 218 (Philippines).

Scelodonta coomani Pic, 1926, Bull. Soc. Zool. Fr. 51: 145 (Tonkin; PARIS). New synonymy. Scelodonta subcostata: Chûjô, 1964, Nature and Life in SE Asia, Kyoto 3: 281, pl. 25, fig. 17 (Thailand).

D istribution : Burma, Nepal, Thailand, Cambodia, Laos, Vietnam, S. China, 
Hainan, Philippines, Singapore, Sumatra, Java, Borneo. England : accidentally introduced.

Material examined. THAILAND: Chieng Dao, 11 exs., 15. vi. 1965, K. Morimoto \& Y. Miyatake; Fang, 4 exs., 13-14. vi. 1965, K. Morimoto \& Y. Miyatake; Khao Chong, nr. Trung, 3 exs., 25-27. vi. 1965, K. Morimoto ; Kor Hong, 1 ex., 23. vi. 1965, K. Morimoto ; Ratapoom, 1 ex., 27. vi. 1965, Y. Miyatake (KU) ; Saraburi, 1 ex., 28. i. 1968, K. Baba (KIMOTO). CAMBODIA: Darmrey Phong, 1 ex., 14-16. iv. 1961, N. R. Spencer ; Ph. Chisau, $40 \mathrm{~km} \mathrm{~S}$ of P. Penh, $20 \mathrm{~m}, 1$ ex., 29. iv. 1961, N. R. Spencer (BISHOP). LAOS: Umg. Vientiane, 4 exs., iiivi. 1963; Umgeb. Vanky, 1 ex., 1963 (MUNCHEN) ; Attopeu Prov., Houei Kong, 3 exs., 3. v. 1965, Native collr ; Ban Van Heue, $20 \mathrm{~km} \mathrm{E} \mathrm{of} \mathrm{Phou-kow-kuei,} 1$ ex., 1-15. v. 1965, J. A. Rondon; Borikhane Prov., Paksane, 1 ex., 3. xii. 1965, Native collr (BISHOP). VIETNAM: $30 \mathrm{~km} \mathrm{NW}$ of Pleiku, $300 \mathrm{~m}, 1$ ex., 10. v. 1960, L. W. Quate (BISHOP) ; Hoa-Binh, Tonkin, 1 ex. (FREY).

Scelodonta granulosa Baly (Fig. 14a)

Scelodonta granulosa Baly, 1867, Trans. Entomol. Soc. Lond., ser. 3, 4: 158 (Borneo, Celebes ; BM) .--Jacoby, 1908, Fauna India, Coleopt. 2: 386 (Madras, Assam). -Gressitt \& Kimoto, 1961, Pac. Insects Monogr. 1A: 243 (S. China).

Scelodonta blaisei Pic, 1926, Bull. Soc. 2001. Fr. 51: 144 (Tonkin; PARIS). New synonymy.

Distribution: India, Thailand, Laos, Vietnam, S. China, Borneo, Celebes.

MAterial EXAmined. THAILAND: S. Banna, Nakhon, 108 m, 1 ex., 5-10. v. 1958, T. C. Maa (BISHOP). LAOS: Attopeu Prov., Houei Kong, 2 exs., 31. v. 1965, Native collr (BISHOP) ; Umg. Vientiane, 1 ex., iii-vi. 1963 (MUNCHEN).

\section{Scelodonta lewisii Baly}

Scelodonta lewisii Baly, 1874, Trans. Entomol. Soc. Lond., 1874: 165 (Japan, Chusan, Shanghai; BM). --Chen, 1935, Sinensia 6 (3) : 328, fig. 25 (S. China) ; 1940, ibid. 11 (5-6) : 485. -Chûjô, 1938, Mushi, Fukuoka 11(2): 162 (Lao Shan) ; 1942, 14(1): 57 (Dairen); 1956, Philip. J. Sci. 85(1): 80 (Formosa). -Gressitt \& Kimoto, 1961, Pac. Insects Monogr. 1A: 243 (China, Hainan). -Kimoto, 1964, J. Fac. Agr. Kyushu Univ. 13(2): 251 (Japan).

Scelodonta orientalis Lefèvre, 1887, Bull. Soc. Entomol. Fr., ser. 6, 7: 56(China). -Fairmaire, 1888, Ann. Soc. Entomol. Belg. 32: 38 (China). -Chen, 1935, Sinensia 6(3): 328 (= lewisii).

Scelodonta jeanvoinei Pic, 1941, Opusc. Mart. 2: 13 (Tonkin; PARIS). New synonymy.

Distribution: Japan, China, Taiwan, Hainan, Vietnam.

No additional material.

Scelodonta vittata (Olivier) (Fig. 14b)

Eumolpus vittata Oliv., 1808, Entomologie 6: 907, pl. 1, fig. 18 (India).

Scelodonta vittata:Lefèvre, 1889, Ann. Soc. Entomol. Fr., ser. 6, 9: 291 (Cochin-Chine: Mytho; Cambodge : Pnomh-Penh) ; 1890, Nouv. Arch. Mus. Paris, ser. 3, 2 : 193 

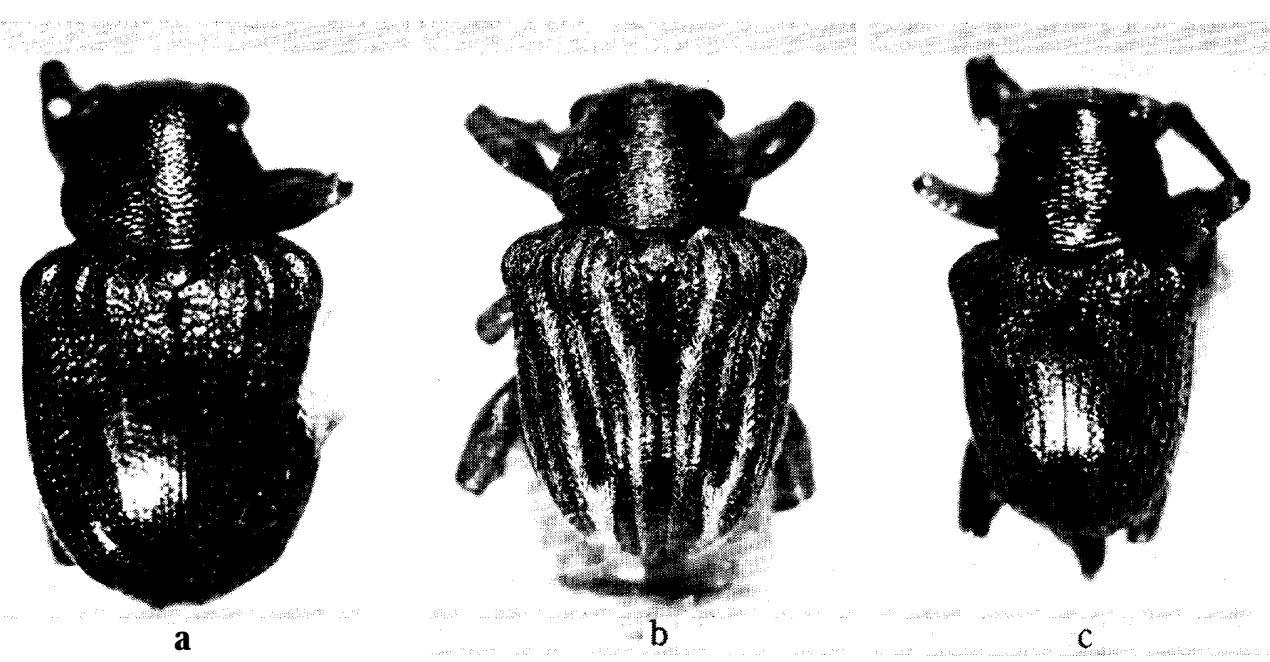

Fig. 14. a, Scelodonta granulosa Baly; b, S. vittata (Olivier); c, S. dillwyni (Stephens).

(Cambodge) ; 1893, Ann. Soc. Entomol. Fr. 1893: 121 (Env. Bangkok) ; 1904, Miss. Pavie Indo-China, ser. 2, 3 (Zool.) : 149 (Cambodge). -Jacoby, 1908, Fauna India, Coleopt. 2: 382, pl. 2, fig. 5 (India, Cochinchina). -Pavia, 1908, Rec. Indian Mus. 2: 404 (India, Burma, Andamans).

Distribution : India, Andamans, Burma, Thailand, Cambodia, Laos, Vietnam.

Material EXAmined. THAILAND: Kanchanaburi, 2 exs., 21. v. 1962 (BANGKHEN). LAOS: Umgeb. Vientiane, 14 exs., iii-vi. 1963(MUNCHEN); Nongtevada, 1 ex., 6. viii. 1965, Native collr (BISHOP). VIETNAM: $22 \mathrm{~km} \mathrm{~S}$ of Trang, 1 ex., 20-26. xi. 1960, C. M. Yoshimoto (BISHOP).

\section{Genus Colasposoma Castelnau}

Colasposoma Castelnau, 1833, in Silbermann, Rev. d'Entomol. 1: 22. --Chapuis, 1874, Genera Coleopt. 10: 301. --Lefèvre, 1885, Mém. Soc. Sci. Liege, ser. 2, 11: 103. -Jacoby, 1908, Fauna India, Coleopt. 2: 439 (type : Colasposoma senegalense Castelnau). -Chen, 1935, Sinensia 6(3): 329; 1940, ibid. $11(5-6): 489$. -Chûjô, 1956, Philip. J. Sci. 85 (1) : 122. Gressitt \& Kimoto, 1961, Pac. Insects Monogr. 1A: 233. -Kimoto, 1964. J. Fac. Agr. Kyushu Univ. 13(2): 249.

Acis Chevrolat, 1837, in Dejean, Cat. Coleopt. ed. 3: 411..--Monrós \& Bechyné, 1956 , Entomol. Arb. Mus. Frey 7: 1127 (type: Colasposoma senegalense Castelnau).

Ballastro Gistl, 1837, Systema Insectorum, 404. -Monrós\& Bechyné, 1956, En tomol. Arb. Mus. Frey 7: 1127.

Thysbe Thompson, 1858, Arch. Entomol. 2: 370.

Palesida Harold, 1874, Berl. Entomol. Z. 18: 23.

Pseudomacetes Linell, 1896, Proc. U. S. Nat. Mus. 18: 695.

Dasychlorus Fairmaire, 1898, Bull. Soc. Entomol. Fr. 1898: 19.

Chiriphyle Jacoby, 1901, Trans. Entomol. Soc. Lond. 1901: 241. 
$1(0)$. Short oval ; antenna robuster, in preapical segments less than three

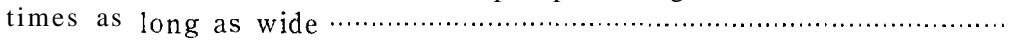

Narrowly oblong; antenna slenderer, in preapical segments nearly three times as long as wide; elytron strongly rugose and tuberculate at side in female and closely transversely rugose but not tuberculate in male ; coloration variable, dorsoal surfaces metallic green, violaceous, blue, or cupreous with margins greenish; antenna and legs brownish in various degrees and in some specimen largely brownish or entirely blackish ; length $4.5-7.2 \mathrm{~mm}$

downesii

2(1). Larger species; antenna about $11 / 2$ times as long as wide in preapical segments ; in male middle tibia strongly curved in apical portion and strongly expanded at apex; lateral portion of elytron obsoletely rugose in male and never rugose in male; dorsum entirely dark blue, violaceous, blue, or violaceous with elytron reddish cupreous; length $6,5-8.2 \mathrm{~mm}$.

pretiosum

Rather small; antenna about twice as long as wide in preapical segments ; in male middle tibia almost straight in apical portion and sligthly expanded at apex; lateral portion of elytron rugose, strongly in female but rather obsoletely in male; coloration varibale, in nominate form head, pronotum, scutellum and ventral surfaces dark blue or violaceous blue, elytron greenish or reddish cupreous, with suture, side and trigonal subbasal marking purplish blue, but in some specimen entirely greenish, bluish, violaceous, cupreous or blackish; length 4.5-G. $5 \mathrm{~mm}$

auripenne

\section{Colasposoma auripenne Motschulsky (Fig. 15a)}

Colasposoma auripenne Motsch., 1860, Schrenck's Reisen Amurl. 2: 179 (Indes oriental). - Jacoby. 1908, Fauna India, Coleopt. 2: 454, pl. 2, fig. 9 (Amur, India, Burma, Andaman). -Gressitt \& Kimoto, 1961, Pac. Insects Monogr. 1A: 235 (China, Hainan).

-Kimoto \& Gressitt, 1966, Pac. Insects 8(2): 506 (Ryukyu Is.). -Kimoto, 1972, Entomol. Ts. 93(4): 145 (Annam).

Colasposoma metallicum Clark, 1865, Ann. Mag. Nat. Hist. ser. 3, 15: 142 (Pulo Penang; BM). -Baly, 1867, Trans. Entomol. Soc. Lond. ser. 3, 4(2): 276 (Pulo Penang). -Jacoby, 1908, Fauna India, Coleopt. 2: 446 (India, Burma, Andaman, Penang). -Gressitt \& Kimoto, 1961, Pac. Insects Monogr. 1A: 236 (China, Hainan). 一Kimoto, 1964, J. Fac. Agr. Kyushu Univ. 13(2): 250 (Japan, Ryukyu Is.). Kimoto\& Gressitt, 1966. Pac. Incects 8(2): 506 (=auripenne).

Colasposoma pulcherrimum Baly, 1864, Descr. n. gen. \&spec. Phytoph., 15 (India). --Jacoby, 1889, Ann. Mus. Civ. Genova 27: 175 (Burma). Lefèvre, 1889, Ann. Soc. Entomol. Fr. ser. 6, 9: 292 (Annam: Hue, Qui-Nhon ; Cochinchina: Saigon, Mythe; Cambodge : Pnomh-Penh) ; 1890, Nouv. Arch. Mus. Paris, ser. 3, 2: 195 (Siam, Cambodge) ; 1904, Fauna India, Coleopt. 2: 454 (=auripenne).

Colasposoma mutabile Baly, 1867, Trans. Entomol. Soc. Lond., ser. 3, 4(2): 273 (Java, Borneo, Malacca, Timor). -Lefèvre, 1887, Notes Leyd. Mus. 9: 261 (Sumatra). -Jacoby, 1908, Fauna India, Coleopt. $2: 454$ (=auripenne).

Colasposoma capitatus Jacoby, 1889, Ann. Mus. Civ. Genova 27: 178 (Burma ; GENOVA) ; 1908, Fauna India, Coleopt. $2: 446$ (=metallicum).

Colasposoma annamita Lefèvre, 3885, Mém.Soc. Sci. Liege, ser. 2, 11: 104, nota 1 (Cochinchine : Saigon) ; 1889, Ann. Soc. Entomol. Fr. ser. 6, 9: 292 (Cochinchine: Saigon) ; 
1893, Ann. Soc. Entomol. Fr. 1893: 126 (Cochinchine). New synonymy.

Colasposoma affine Lefèvre, 1890, Neuv. Arch. Mus. Paris, ser. 3, 2: 195 (Laos: LuangPrabang; PARIS) ; 1904, Miss. PavieIndo-China, ser. 2, 3 (Zool.) : 151 (Laos). New synonymy.

Colasposoma oberthuri Jacoby, 1896, Entomologist 29: 6 (Amami-Oshima; cotype :BRUXELES)._Chûjô, 1956, Philip. J. Sci. 85(1): 124, fig. 3 (Formosa). --Kimoto, 1964, J. Fac. Agr. Kyushu Univ. 13(2): 250 (=metallicum).

Colasposoma auripenne ab. sumatrense Weise, 1926, Treubia 8: 243 (Sumatra).

Colasposoma jeanvoinei Pic, 1937, Mel. Exot. Entomol. 69: 21 (Tonkin; PARIS). New synonymy.

Colasposoma brevenotatum Pic, 1937, Mél. Exot. Entomol. 69: 22 (Tonkin; PARIS). New synonymy.

Colasposoma perroudi Pic, 1937, Mel. Exot. Entomol. 69: 22 (Cochin China ; PARIS). New synonymy.

Colasposoma oberthuri abb. aurita,azurea,azureolineata,bryanti,chujoi, sauteri, violacea Papp, 1946, Additam. Fauna. Coleopt.4(1): 7 (locality not cited).

Distribution : India, Burma, Andamans, Thailand, Laos, Cambodia, Vietnam, Hainan, S. China, Ryukyus, Taiwan, Malaya, Sumatra, Java, Borneo, Molluccas, Timor.

Material examined. THAilAnD: Kanchanaburi, 5 exs., 21. v. 1962, 4 exs., 31. v. 1962; San Ngow Tak, 1 ex., 6. vi. 1959 (BANGKHEN) ; Tang Prov., Water Fall to Khaochang, 150-300 m, 2 exs., 3. i. 1964, G. A. Samuelson; Chanthabiri, Prew, 2 exs., 24. iv. -1. v. 1958, T. C. Maa; Chiang Mai Prov., Mae Klang, 1 ex., 11. vi. 1965, P. D. Ashlock; Chiang Dao, 1 ex., vi. 1965, P. D. Ashlock (BISHOP) ; Chieng Dao, 3 exs., 15. vi. 1965, K. Morimoto \& Y. Miyatake, 2 exs., 23. iv. 1973, Y. Yoshiyasu; Mae Sa Water Fall, 1 ex., 11. vi. 1965, 1 ex., 16. vi. 1965, K. Morimoto; Fang, 1 ex., 22. viii. 1973, K. Yano ; Mae Chan, 1 ex., 13. vii. 1970, K. Yano; Samptong, 1 ex., 14. vii. 1973, S. Nakano ; Khao Yai Nat. Park, 2 exs., 5. vi. 1965, S. Asahina \& K. Morimoto; Muak Lek, $40 \mathrm{~km} \mathrm{SE}$ of Saraburi, 1 ex., 5. vi. 1965, Y. Miyatake; Prew, Chanta Buri, 2 exs., 29. viii. 1973, S. Nakao; Khao Chong, nr. Trung, 3 exs., 26-27. vi. 1965, K. Morimoto \& Y. Miyatkae (KU). LAOS: Umgeb. Vientiane, 56 exs., iii-vi. 1963; Umgeb. Paklay, 24 exs., 1963, 1 ex., 1964; Umgeb. Pakse, 8 exs., 1963; Umgeb. Vanky, 3 exs., 1963, 4 exs., 1964 (MUNCHEN) ; Ban Van Heue, 20 km E of Phou-kowkuei, 2 exs., 15-31. v. 1965, Native collr; Lunag Prabang, 300 m, 1 ex., 4-5, v. 1960, 1 ex., 11-12. vi. 1960, S. Quate; Ile de Khong, 1 ex., 7. ix. 1965, Native collr ; Borikhane Prov., Pakkading, 1 ex., 10. v. 1965, 4 exs., 31. vii. 1961, 1 ex., 3. viii. 1965, 1 ex., 15. vi. 1966, Native collr; Attopeu Prov., Houei Kong, 4 exs., 31. v. 1965, 1 ex., 20. vii. 1965, Native collr; Sayaboury Prov., Sayaboury, 3 exs., 8. v. 1965, 1 ex., 5. v. 1966, Native collr; Vientiane Prov., Vientiane, 1 ex., 31. v. -3. vi. 1960, L. W. Quate, 1 ex., 22. vii. 1965, Native collr; Sedone Prov., Pakse, 1 ex., 14. v. 1965, P. D. Ashlock, 1 ex., 5. vii. 1965, Native collr; Paksong, 1 ex., 16. 1965, Native collr (BISHOP). VIETNAM: Ban Me Thuot, 500 m, 15 exs., 16-18. v. 1960, S. Quate, L. W. Quate \& R. E. Leech; $33 \mathrm{~km} \mathrm{NE}$ of Ban Me Thuot, 870 m, 1 ex., 18. v. 1960, L. W. Quate; M'Drak, E of Ban 

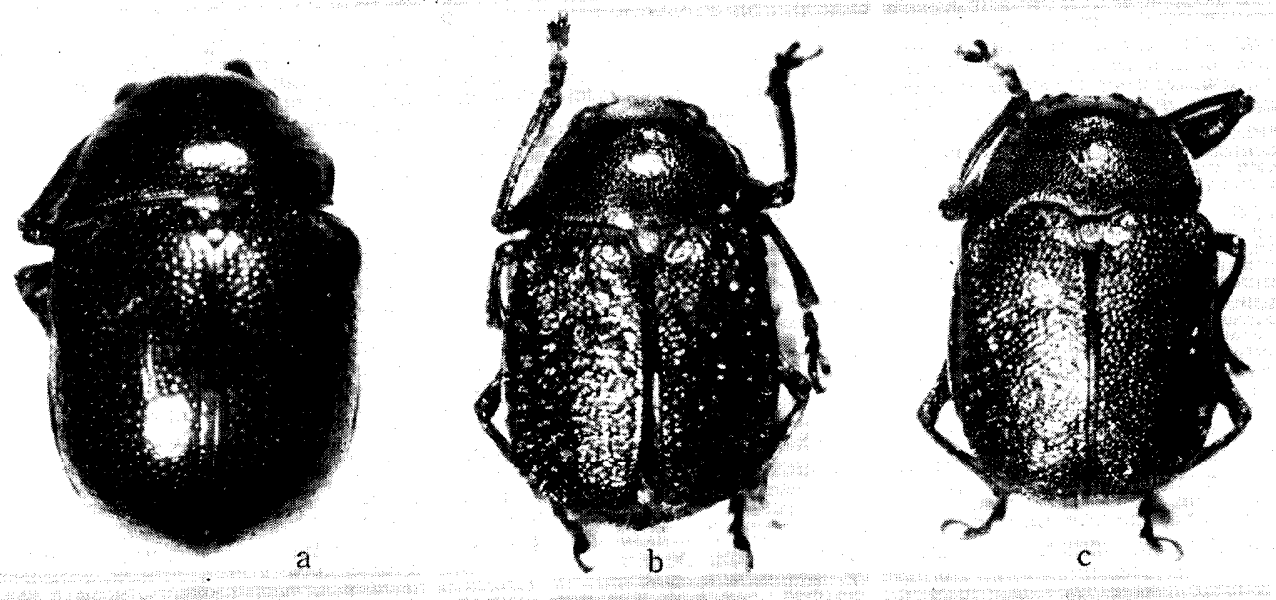

Fig. 15. a, Colasposoma auripenne Motschulsky; b, C. duwnesii Baly, female ; c, ditto, male.

Me Thuot, 4-600 m, 1 ex., 8-19. xii. 1960, L. W. Quate; Nha Ho, 14 km N Phan Ran, 2 exs., 6. xi. 1960, 2 exs., 15. xi. 1960, C. M. Yoshimoto; Dilinh (Djiring), $1200 \mathrm{~m}, 1$ ex., 22.28. iv. 1960, S. Quate; $28 \mathrm{~km} \mathrm{~N}$ of Dilinh, $900 \mathrm{~m}, 1$ ex., 22-28. iv. 1960, L. W. Quate; $40 \mathrm{~km} \mathrm{~N}$ of Dilinh, $540 \mathrm{~m}, 1$ ex., 26. iv. 1968, R. E. Leech (BISHOP) ; Riv. de Hue, Annam, 1 ex., 16. iii. 1927, Mrs Dora \& E. Wright (CAS) ; Hoa-Binh, Tonkin, 70 exs., A. Cooman;Chapa,Tonkin, 4 exs. (FREY).

\section{Colasposoma downesii Baly (Figs. 1c, 15b-c)}

Colasposoma downesii Baly, 1862, Ann. Mag. Nat. Hist. ser. 3, 10: 19 (India ; BM). -Jacoby, 1908, Fauna India, Coleopt. 2: 442, pl. 2, fig. 7 (India, Sikkim, Burma). -Kimoto, 1970, Spec. Bull. Lepidop. Soc. Japan, 4: 165 (Nepal).

Colasposoma aureovittatum Baly, 1864, Descr. n. gen. \& spec. Phytoph., 14 (India; BM). Lefèvre, 1893, Ann. Soc. Entomol. Fr. 62: 126 (Tonkin). Jacoby, 1908, Fauna India, Coleopt. 2: 455 (India). New synonymy.

Colasposomaigneicolle Baly, 1865, Trans. Entomol. Soc. Lond., ser. 3, 2(5): 428 (Siam ; BM). New synonymy.

Colasposoma viridiaeneum Baly, 1865, Trans. Entomol. Soc. Lond., ser. 3, 2(5): 429 (Laos; $\mathrm{BM})$. N ew synonymy.

Colusposoma ornaticolle Jacoby, 1881, Proc. Zool. Soc. Lond., 1881: 444 (Cochinchina). --Lefèvre, 1890, Nouv. Arch. Mus. Paris, ser. 3, 2: 195 (Siam : Battambang; Cambodge) ; 1904, Miss. Pavie Indo-China, ser. 2, 3 (2001.) : 151 (Siam, Cambodge). New synonmy.

Colasposoma asperatumLefèvre, 1885, Mém. Soc. Sci Liège, ser. 2, 11: 104, nota 2 (Cochinchina: Saigon) ; 1889, Ann. Soc. Entomol. Fr. ser. 6, 9: 292 (Cochinchina: Saigon) ; 1890, Nouv. Arch. Mus. Paris, ser. 3, 2: 195 (=ornaticoite).-Jacoby, 1895, Ann. Soc. Entomol. Belg. 39: 274 (good species). -Lefèvre, 1904, Miss. Pavie Indo-China, ser. 2, 3 (Zool.): 151 (=ornaticolle).-Jacoby, 1908, Fauna India, Coleopt. 2: 452, fig. 156 (India, Burma to China). -Gressitt \& Kimoto, 1961, Pac. Insects Mongor. 1A: 237 ("China"). - Chû́jô, 1964, Nature and Life in SE Asia, Kyoto 3: 270 (Thailand).

Colasposoma transversicolle Jacoby, 1889, Ann. Mus. Civ. Genova 27: 176 (Burma; GENOVA). -Lefèvre, 1893, Ann. Soc. Entomol. Fr. 189: 126 (Tonkin). -Jacoby, 1908, Fauna 
India, Coleopt. 2: 448 (Burma). New synonmy.

Colasposoma multipunctata Jacoby, 1889, Ann. Mus. Civ. Genova 27 : 177 (Burma ; GENOVA) ; 1895, Ann. Soc. Entomol. Belg. 39: 274 (=asperatum); 1908, Fauna India, Coleopt. 2: 448 (Burma).

Colasposoma angulicolle Le fè vre, 1893, Ann. Soc. Entomol. Fr. 62: 127 (Cochinchina ; PARIS). New synonymy.

Colasposoma insuturatum Pic, 1937, Mel. Exot. Entomol. 69: 21 (Tonkin: Lac Tho ; PARIS). New synonymy.

Colasposoma saigonense Pic, 1937, Mél. Exot. Entomol. 69: 22 (Saigon ; PARIS). New synonymy.

Colasposoma downesi var. purpureicolle Pic, 1937, Mél. Exot. Entomol. 69: 22 (Cambodia).

Distribution : India, Nepal, Burma, Thailand, Laos, Cambodia, Vietnam.

Material ExAmined. THAILAND: Uthaithai, 3 exs., 13. iv. 1963 (BANGKHEN) ; Chiang Mai Prov., Mae Sa, 1 ex., 16. vi. 1965, P. D. Ashlock; Chiang Dao, 1 ex., 15. vi. 1965, P. D. Ashlock (BISHOP) ; Doi Suthep, 3 exs., 8. vi. 1965, 3 exs., 10. vi. 1965, 3 exs., 12. vi. 1965, K. Morimoto; Tan Keo, Doi Suthep, 5 exs., 10. vi. 1965, Y. Miyatake; Mae Klang Water Fall, nr. Chom Thong; 3 exs., 11. vi. 1965, K. Morimoto \& Y. Miyatake; Fang, 1 ex., 13. vi. 1965, K. Morimoto; Pak Chong, Korat Plateau, 1 ex., 14. iv. 1973, Y. Yoshiyasu (KU). LAOS: Umgeb. Vientiane, 29 exs., iii-vi. 1963, Umgeb. Pakse, 8 exs., 1963; Umgeb. Paklay, 4 exs., 1963; Umgeb. Vanky, 2 exs., 1964 (MUNCHEN) ; Muong Sing, NW of Luang Prabang, 650 m, 1 ex., 6 -10. vi. 1960, L. W. Quate ; Ban Van Heue, $20 \mathrm{~km}$ E of Phou-kow-kuei, 1 ex., 1-15. v. 1965, J. A. Rondon; Borikhane Prov., Pakkading, 1 ex., 22. iii. 1965, 1 ex., 15. vi. 1966, Native collr; Wapikhamthong Prov., Khong Sedone, 1 ex., 16. v. 1965, 1 ex., 30. v. 1965, Native collr ; Khammoune Prov., Phon Tiou, 2 exs., 17. v. 1965, Native collr ; Vientiane Prov., Ban Van Eue, 2 exs., 15. v. 1965, Native collr; Vientiane, 1 ex., 31. v. -3. vi. 1960, S. Quate; Sedone Prov., Pakse, 1 ex., 14. v. 1965, 7 exs., 15. v. 1965, 3 exs., 23. v. 1965, 1 ex., 24. v. 1965, P. D. Ashlok ; $5 \mathrm{~km}$ E of Pakse, 1 ex., 13. v. 1965, P. D. Ashlock; Ile de Khong, 6 exs., 7. 1965, 2 exs., 17. v. 1965, Native collr (BISHOP). VIETNAM: $50 \mathrm{~km}$ SE of Pleiku, 5 exs., 11. v. 1960, 4 exs., 14. v. 1960, L. W. Quate; $20 \mathrm{~km} \mathrm{~N}$ of Pleiku, 4 exs., 9. v. 1960, L. W. Quate (BISHOP).

\section{Colasposoma pretiosum Baly}

Colasposoma pretiosum Baly, 1860, J. Entomol. 1: 36 (India ; BM). Jacoby, 1908, Fauna India, Coleopt. 2: 449, pl. 2, fig. 8 (Darjeeling, Assam, Tibet). Gressitt \& Kimoto, 1961, Pac. Insects Monogr. 1A: 234 (China).

Colasposoma coeruleatum Baly, 1879, Cistula Entomol. 2: 440 (Assam ; BM). -Gressitt \& Kimoto, 1961, Pac. Insects Monogr. 1A:234 (=pretiosum).

Colasposoma thibetanum Jacoby, 1891, Entomologist 24 (Suppl.) : 35 (Tibet; BM) ; 1908, Fauna India, Coleopt. 2: 449 (=pretiosum).

Colasposoma purpureum Pic, 1937, Mel. Exot. Entomol. 69: 21 (Yunnan; PARIS). -Gressitt \& Kimoto, 1961, Pac. Insects Monogr. 1A:234 (=pretisosum).

Distribution : India, Burma, Thailand, Laos, Vietnam, China. 

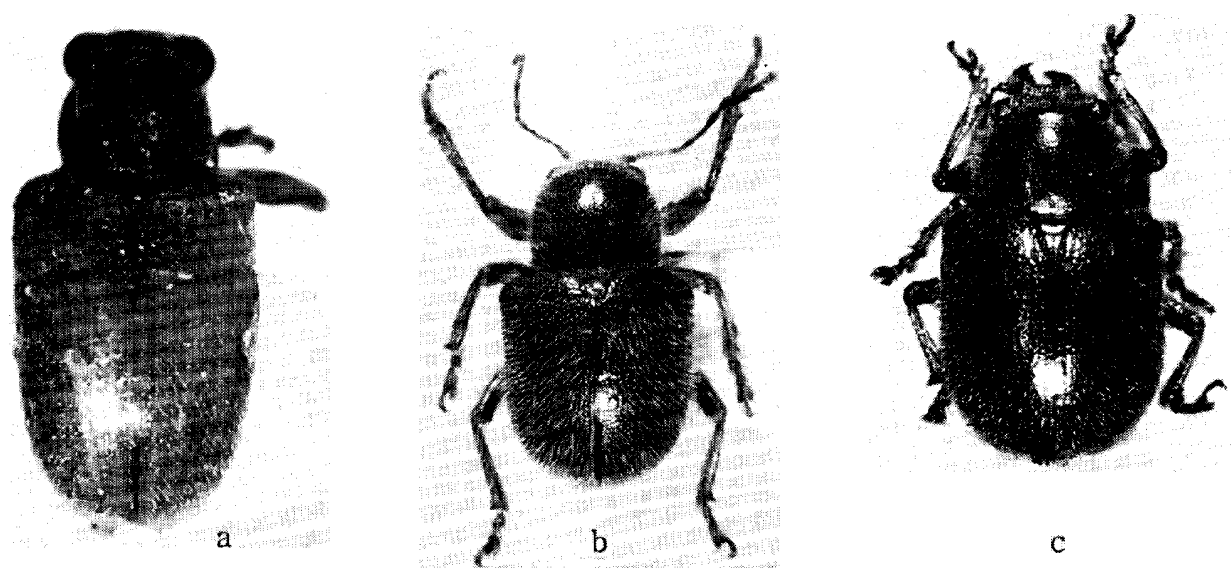

Fig. 16. a, O snaparis pallidipennis (Pic); b, Trichochrysea hirfa (Fabricius) ; c, T. mouhoti Baly.

Material examined. Thailand: Kanchanaburi, 4 exs., 21. v. 1962 (BANG KHEN). LAOS: Umgeb. Vientiane, 23 exs., iii-vi. 1963; Umgeb. Paklay, 8 exs., 1963 ; Umgeb. Vanky, 3 exs., 1963, 5 exs., 1964 (MUNCHEN) ; Muong Sing, NW of Luang Prabang, 650 m, 2 exs., 6-10. vi. 1960, S. Quate \& L. W. Quate; Ban Van Heue, $20 \mathrm{~km}$ E of Phou-kow-kuei, 1 ex., 15-31. v. 1965, Native collr; Vientiane Prov., Ban Van Eue, 1 ex., 30. vi. 1966, 1 ex., 15. vii. 1966, Native collr; Sedone Prov., Pakse, 1 ex., 17. vii. 1965, Native collr ; Attopeu, Houei Khong, 2 exs., 16. v. 1965, Native collr; Borikhane Prov., Pakkading, 1 ex., 22. iii. 1965, 1 ex., 10. v. 1965, 1 ex., 9. ix. 1965, Native collr (BISHOP). VIETNAM: Hoa-Binh, Tonkin, 1 ex., A. Cooman (FREY).

\section{Genus Osnaparis Fairmaire}

O snaparis Fairmaire, 1889, Ann. Soc. Entomol. Fr., 1889: 72 (type : O snaparis nucea Fairmaire, from Moupin). -Chûjô, 1956, Philip. J. Sci. 85(1):113. -Gressitt \& Kimoto, 1961, Pac. Insects Monogr. 1A: 266. - Kimoto, 1964, J. Fac. Agr. Kyushu Univ. 13(2): ' 260.

$$
\text { K EY TO SPECIES OF Oshaparis }
$$

Tibiae and apex of femora blackish; reddish brown, antenna blackish with basal segments brownish ; length 5.5-6.7 mm (Fairmaire, 1889 ; China, Taiwan, Japan)

nucea

Tibiae blackish with basal portion brownish; yellowish to reddish brown; antenna blackish with basal segments brownish; punctures of dorsum finer; length

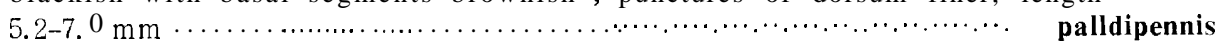

Osnuparis pallidipennis (Pic) New combination (Fig. 16a)

Aoria pallidipennis Pic, 1928, Bull.Soc. Zool. Fr., 53 : 377 (Tonkin; PARIS). 
Aoria tonkinea Pic, 1935, Mél. Exot. Entomol. 66: 21 (Tonkin:Chapa; PARIS). New synonymy.

D istribution: Thailand, Vietnam.

Material examined. Thailand :Doi Pui, 1685 m, Chiang Mai Prov., 1 ex., 17. vi. 1965, Y. Miyatake (KU).

\section{Genus Trichochrysea Baly}

Trichochrysea Baly, 1861, J. Entomol. 1: 195 (type: Trichochrysea mouhoti Baly, from Cambodia). -Jacoby, 1908, Fauna India, Coleopt. 2: 387. -Chen, 1935, Sinensia 6 (3) : 235; 1940, ibid. 11 (5-6) : 490. —Chûjô, 1956, Philip. J. Sci. 85 (1) : 83. -Gressitt \& Kimoto, 1961, Pac. Insects Monogr. 1A: 244. -Kimoto, 1964, J. Fac. Agr. Kyushu Univ. 13(2): 252.

\section{Key to SPECIES OF Trichochrysea}

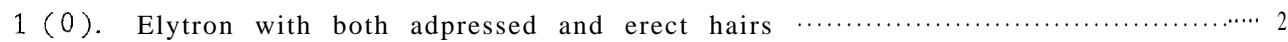

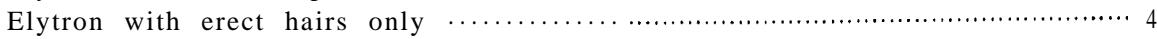

2(1). Pronotum rugosely and closely impressed by deep, elongate punctures;

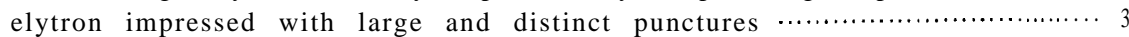

Pronotum with surface smooth, finely impressed by minute, round punctures; elytron impressed with fine punctures and their interstices

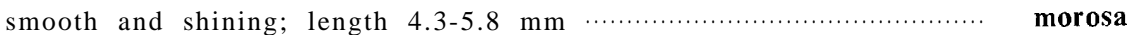

3 (2). Interstices of elytral punctures flat or slightly raised, but not irregular;

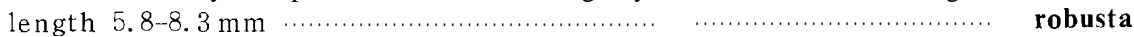

Interstices of elytral punctures irregularly convex ; length 8.3-9.0 mm ….........

4 (1). Pronotum entirely reddish cupreous ; antenna with apical segments more

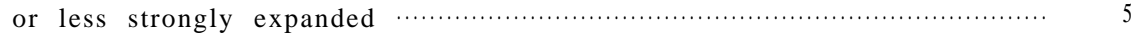

Not as above combination of characters ..................................... 7

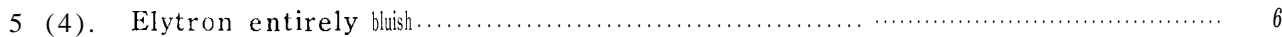

Elytron greenish with a large, oblique, reddish curpreous marking which is narrow basally and widened posteriorly; length $5.9-7.6 \mathrm{~mm} \cdots \ldots . . .$. vitalisi

6 (5). Pronotum with anterior corner sharply pointed and produced' laterally; violaceous blue, head and pronotum reddish cupreous ; length 5.3-

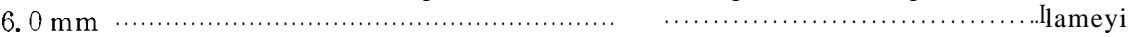

Pronotum with anterior corner not pointed nor produced laterally; violaceous blue, pronotum and posterior half of head reddish cupreous;

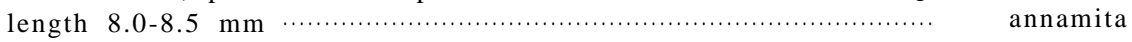

7 (4). Antenna with apical segments more or less strongly widened and flat-

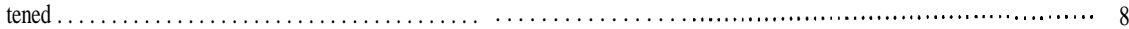

Antenna slender, filiform, apical segments not distinctly widened and

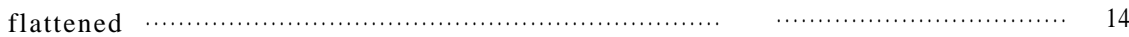

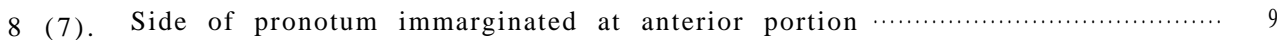

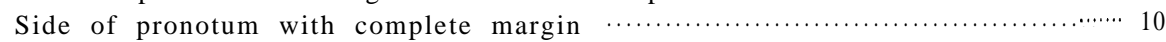

9 (8). Anterior margin of clypeus with a strongly produced sharp tooth in each side in male and slightly produced broad tooth in female; dorsal surfaces covered with both whitish and blackish hairs; coloration variable: 1) head red cupreous with marginal portion greenish, pronotum red cupreous with a pair of triangular markings united at middle gold- 
en green, elytron red cupreous with subbasal transverse band and subapical elongate marking, together with entire lateral, apical and sutural margins and most of basal margin, golden green, ventral surfaces mostly red cupreous; 2) ground color cupreous green to golden green with subbasal transverse band and subapical elongate marking bluish, ventral surfaces mostly golden green; 3) entirely blackish to violaceous blue; length $9.2-11.9 \mathrm{~mm}$

Anterior margin of clypeus without any distinct tooth in side; dorsal surfaces covered with blackish hairs only; general color greenish blue, violaceous or purple; length $9.5-12.5 \mathrm{~mm}$

imperialis

10 (8). Elytron without distinct marking dorsally

Elytron violaceous blue with basal half and apical marking reddish cupreous; short ovate, general color violaceous blue ; length $6.0-7.4 \mathrm{~mm} \ldots$ hebe

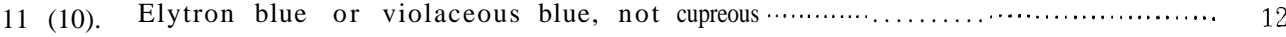

Elytron greenish to reddish or purplish cupreous; general color violaceous or violaceous blue; scutellum sparingly punctate ; length 7.8-9.5

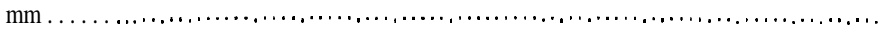

aeneipennis

12 (11). 9th and 10th segments of antenna each slightly longer than broad $\cdots \cdots \cdots \cdots \cdots \cdots \cdots \cdots$ 9 th and 10th segments of antenna transversal; general color violaceous blue; length $7.8-8.5 \mathrm{~mm}$

tarsata

13 (12). Oblong-ovale, elytron subparallel sided and rounded at apex; dark blue;

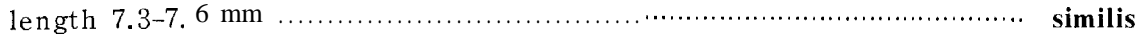

Short ovate ; elytron widest at humerus and gradually narrowed behind

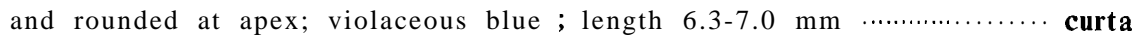

14 (7). Anterior margin of clypeus deeply and widely rounded . . . . . ...................... 15

Anterior margin of clypeus nearly straight, pronotum slightly wider than long and nearly $11 / 5$ times as wide as long; general color blackish blue, reddish cupreous or golden green ; length $5.8-8.3 \mathrm{~mm} \ldots \ldots \ldots \ldots$ mandarina

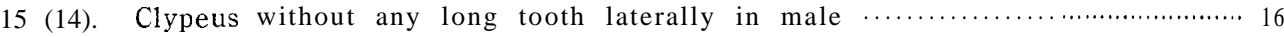

Clypeus with a long tooth laterally in male; pronotum transversely shaped, quite twice as broad as long, surface extremely closely and strongly punctate, punctures nearly confluent at side ; obscure aeneous,

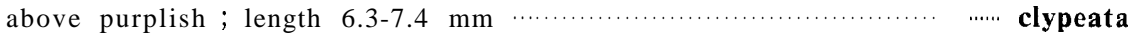

16 (15). Antenna nearly three times as wide as long in preapical segments $\ldots \ldots \ldots \ldots \ldots \ldots \ldots \ldots$

Antenna slenderer, nearly four times as long as wide in preapical segments ; body relatively narrow; labrumpiceous; pronotum nearly $11 / 5$ times as wide as long; diameter of punctures generally narrower than their interstices; general color blackish blue, violaceous or greenish blue ; length 5.2-5. $9 \mathrm{~mm}$

17 (16). Pronotum widest slightly behind anterior margin and strongly narrowed

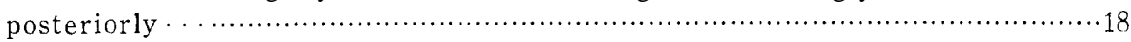

Pronotum widest slightly before middle and narrowed anteriorly and

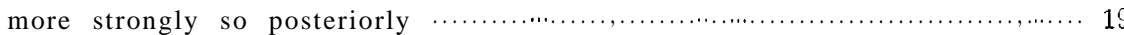

18 (17). Dorsal surfaces violaceous, blackish blue or greenish blue; length 8. 0 $8.3 \mathrm{~mm}$

Dorsal surfaces bluish green, with elytral humerus cupreous ; length 8.0

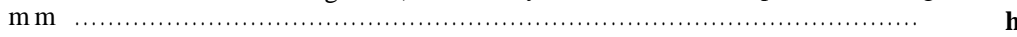

19(17). Pronotum with slightly raised latero-anterior tubercle which is covered with distinct punctures and fine hairs; punctures of pronotum stronger, diameter of punctures nearly twice as wide as their interstices; dorsal surfaces bluish green, violaceous blue or greenish cupreous; length $5.5-7.4 \mathrm{~mm}$ 
Pronotum with distinctly raised latero-anterior tubercle which is smooth, shining and without distinct punctures; punctures of pronotum distinct, diameter of punctures nearly as wide as their intersirces; body broad, labrum bluish, violaceous or greenish; pronotum transverse. nearly $11 / 3$ times as wide as long. widest slightly before middle and narrowed anteriorly and more strongly so posteriorly; general color blackish blue, violaceous or golden green; length $6.1-10.0 \mathrm{~mm}$

hirta

\section{Trichochrysea aeneipennis (Lefèvre) New combination}

Heteraspis aeneipennisLefèvre, 1890, Nouv. Arch. Mus. Paris, ser. 3, 2: 194 (Laos; PARIS) ; 1904, Miss. Pavie Indo-China, ser. 2, 3 (Zool.) : 150 (Laos).

Heteraspis gloriosa Lef., 1893, Ann. Soc. Entomol. Fr. 62: 123 (Tonkin; PARIS). New synonymy.

Trichochrysea gloriosa: Chen, 1935, Sinensia 6 (3) : 341 (S. China, Tonkin). -Gressitt \& Kimoto, 1961, Pac. Insects Monogr. 1A: 246 (Tonkin).

Distribution: S. China, Vietnam.

No additional material.

\section{Trichochrysea annamita (Lefèvre)}

Heteraspis annamita Lef., 1877, Ann. Soc. Entomol. Fr., ser. 5, 7: 309 (Cochinchina, Hongkong).

Trichochrysea annamita: Gressitt \& Kimoto, 1961, Pac. Insects Monogr. 1A: 245 (S. China, Vietnam).

Distriburion: S. China, Vietnam.

No additional material.

\section{Trichochrysea bidens (Lefèvre)}

Heteraspis bidens Lef., 1893, Ann. Soc. Entomol. Fr. 62: 124 (Tonkin).

Trichochrysea bedoci Pic, 1926, Mél. Exot. Entomol. 46: 11(Tonkin; PARIS). N ew synonymy.

Trichochrysea bidens: Chen, 1935, Sinensia 6 (3): 344 (Tonkin). -Gressitt \& Kimoto, 1961, Insects Monogr. 1A: 245 (Tonkin).

Distribution : Thailand, Laos, Vietnam.

We could not trace the type location of this species. According to the redescription made by Chen (1935), this species seems to be identical with Trichochrysea bedoci $\mathrm{P}$ ic.

No additional material.

\section{Trichochrysea cephalotes (Lefèvre)}

Heteraspis cephalotes Lef., 1893, Ann. Soc. Entomol. Fr. 62: 124 (Tonkin; PARIS),

Trichochrysea cephalotes : Chen, 1935, Sinensia 6 (3) : 345 (Tonkin).

Trichochrysea viridipesPic, 1936, Mél. Exot. Entomol. 67: 14 (Tonkin ; PARIS). New synony-

my.

Distribution: Vietnam.

No additional material. 
Trichochrysea clypea ta (Jacoby)

Heteraspisclypeata Jacoby, 1889, Ann. Mus. Civ. Genova, 27: 172 (Burma ; GENOVA, BM). LefPvre, 1893, Ann. Soc. Entomol. Fr. 1893: 124 (Tonkin).

Trichochrysea clypeata: Jacoby, 1908, Fauna India, Coleopt. 2: 391, fig. 13 (Burma). Chen, 1935, Sinensia 6 (3): 345 (Yunnan, Vietnam). Gressitt\& Kimoto, 1961, Pac. Insects Monogr. 1A: 246 (Yunnan, Vietnam).

Distribution : Burma, Vietnam, China (Yunnan).

No additional material.

\section{Trichochrysea curta $\mathrm{Pic}$}

Trichochrysea curta Pic, 1926, Mill. Exot. Entomol. 46: 12 (Tonkin; PARIS).

Distribution : Thailand, Laos, Vietnam.

This species might be proved as an infraspecific variation of Trichochrysea hebe (Baly) in future.

Material examined. Thailand: Fang, 1 ex., 14. vi 1965, K. Morimoto (KU). LAOS : Savannakhet Prov., Savannakhet, 1 ex., 20. vii. 1965, Native collr (BISHOP). VIETNAM: $20 \mathrm{~km} \mathrm{~N}$ of Pleiku, $650 \mathrm{~m}, 1$ ex., 9. v. 1960, S. Quate (BISHOP).

Trichochrysea hebe (Baly) (Fig. 18a)

Bromius hebe Baly, 1864, J. Entomol. 2: 220 (Siam; BM).

Heteraspishebe: Jacoby, 1889, Ann. Mus. Civ. Genova 27: 173 (Burma). LefPvre, 1890, Nouv. Arch. Mus. Paris. ser. 3, 2:194 (Laos) ; 1909, Miss. Pavie Indo-China, ser. 2, 3 (Zool.): 150 (Laos).

Trichochrysea hebe: Jacoby, 1908, Fauna India, Coleopt. 2: 393 (Burma, Siam).

Trichochrysea splendida Achard, 1921, Bull. Soc. Entomol. Fr., 1921: 171 (Laos:Luang-Prabang). New synonymy.

Distribution : Burma, Thailand, Laos.

We could not trace the type location of Trichochrysea splendida Achard. Judging from the original description, $T$. splendida is no doubt identical with hebe (Baly), in having the characteristic coloration and marking of the dorsal surfaces.

Material examined. LaOS: Umg. Vientiane. 3 exs., iii vi. 1963, Umgeb. Paklay, 1 ex.. 1963 (MUNCHEN) ; Vientiane Prov., Ban Van Eue, 1 ex., 30. ix. 1965, Native collr (BISHOP).

Trichochrysea hirta (Fabricius) (Fig. 16b)

Eumolpus hirtus Fabr., 1801, Syst. Eleuth. 1: 420 (Sumatra).

Bromius hirtus: Baly, 1867, Trans. Entomol. Soc. Lond., ser. 3, 4: 96 (Malacca, Tringanee, Penang, Sumatra, Java, Sylhet).

Heteraspis nitidissima Jacoby, 1888, Proc.Zool.Soc. Lond., 1888: 345 (China ; BM, MCZ). LefPvre, 1893, Ann. Soc. Entomol. Fr. 1893: 125 (Cambodge: Luang Prabang). New synonymy. 
Heteraspis vestita: Lefèvre, 1890, Nouv. Arch. Mus. Paris, ser. 3, 2: 194 (Laos) ; 1909 , Miss. Pavie Indo-China, ser. 3, 3 (2001.) : 150 (Laos).

Heteraspis viridis Jacoby. 1890, Entomologist, 1892 (Suppl.) : 86 (Burma ; BM). New synonymy. Trichochrysea nitidissima :Lefèvre, 1893, Ann. Soc. Entomol. Fr. 62: 125 (Cambodge). -Chen, 1935, Sinensia 6 (3) : 343 (China). -Gressitt \& Kimoto, 1961, Pac. Insects Monogr. 1A: 247 (China).

Trichochrysea hirta: Jacoby, 1908, Fauna India, Coleopt, 2: 390 (Sylhet, Malayan Is.). -Kimoto, 1972, Entomol. Ts. 93 (4) : 145 (Annam).

Trichochrysea viridis : Jacoby, 1908, Fauna India, Coleopt. 2: 392 (Burma). --Chen, 1940, Sinensia 11 (5-6) : 507 (China).-Gressitt \& Kimoto, 1961, Pac. Insects Monogr. 1A: 245 (Burma).

Trichochrysea scutellaris Pic, 1926, Mél. Exot. Entomol. 46: 11 (Hoa-Binh ; PARIS) .-Gressitt \& Kimoto, 1961, Pac. Insects Monogr. 1A: 248 (= nitidissima).

Trichochrysea donckieri Pic, 1926, Mél. Exot. Entomol. 46: 11 (Tonkin; PARIS). _Gressitt\& Kimoto, 1961, Pac. Insects Monogr. 1A : 248 (= nitidissima).

Trichochrysea diversicolor Pic, 1939, VII Int. Kongr. Entomol. Verh. 1: 344 (China; PARIS). -Gressitt \& Kimoto, 1961, Pac. Insects Monogr. 1A: 247 (=nitidissima).

Distribution : India, Burma, Thailand, Cambodia, Laos, Vietnam, Hainan, S. China, Malaya, Sumatra, Java.

According to the study on the material preserved in Paris Museum, the identification of Trichochrysea vestita by Lefèvre $(1890,1909)$ should be corrected as hirta. T. vestita is separable from hirta in having clypeus of male produced at either side into a strong tooth, and the dorsal surfaces always dark cupreo-aeneous.

Material examined. THAiland: Kanchanaburi, $1300 \mathrm{ft}, 6$ exs., 21. v. 1962 (BANGKHEM) ; Chiangmai Prov., Fang (Agr. Expt. Stat.), $600 \mathrm{~m}, 1$ ex., 14. vi. 1965, P. D. Ashlock (BISHOP) ; Fang, 3 exs., 14. vi. 1965, K. Morimoto ; Doi Suthep, $1000 \mathrm{~m}, 1$ ex., 12. vi. 1965, 1 ex., 19. vi. 1965, Y. Miyatake (KU). LAOS: Umgeb. Vientiane, 53 exs., iii-vi. 1963; Umgeb. Pakse 5 exs., 1963; Umgeb. Paklay, 3 exs., 1963; Umgeb. Vanky, 1 ex., 1963, 3 exs., 1964 (MUNCHEM); Bori- khane Prov., Pakkading, 2 exs., 17. v. 1965, Native collr; Khammouane Prov., Phon Tiou, 4 exs., 17. v. 1965, Native collr ; Sedone Prov., Paksong, 2 exs., 16. v. 1965, Native collr ; Vientiane Prov., Ban Van Eue, 1 ex., 15.v. 1966, Native collr; Ban Van Heue, 20 km E of Phou-kow-kuei, 1 ex., l-15. v. 1965, J. A. Rondon, 1 ex., 15-31. v. 1965, Native collr (BISHOP). VIETNAM: $7 \mathrm{~km}$ SE of Dilinh (Djiring), 990 m, 1 ex., 2. v. 1960, R. E. Leech; $28 \mathrm{~km} \mathrm{~N}$ of Dilinh, $900 \mathrm{~m}$, 1 ex., 22-28. iv. 1960, L. W. Quate (BISHOP) ; Hoa-Binh, Tonkin, 2 exs., A. Cooman (FREY).

\section{Trichochrysea humeralis Pic}

Trichochrysea humeralis Pic, 1928, Bull. Soc. 2001. Fr., 53: 378 (Tonkin: Hoa-Binh ; PARIS).

Distribution : Vietnam.

It is possible that this species is nothing but a infraspecific variation of

$\mathrm{T}$. cephalotes (Lefèvre).

No additional material. 
Trichochrysea imperialis (Baly) (Fig. 2b)

Callomorphaimperialis Baly, 1861. J. Entomol. 1: 285 (N. China ; BM).

Bromius imperialis: Baly, 1865, J. Entomol. 2: 440 (N. China).

Heteraspis imperialis: Jacoby, 1888, Proc. 2001. Soc. Lond. 1888: 345 (China). -Lefèvre, 1893, Ann. Soc. Entomol. Fr. 1893: 123 (Tonkin, Lang-Son, Ha-Lang).

Trichochrysea imperialis: Achard, 1921, Bull.Soc. Entomol. Fr. 1921: 172 (key). --Chen, 1935, Sinensia 6 (3) : 339, fig. 28 (China, Tonkin). Gressitt \& Kimoto, 1961, Pac. Insects Monogr. 1A: 246 (China).

Trichochrysea reitteri Pic, 1939, Entomol. Nachr.B1. 12: 158 ("NW China"; PARIS). -Gressitt \& Kimoto, 1961, Pac. Insects Monogr. 1A: 246 ( $=$ imperialis).

Distribution : S. China, Vietnam.

No additional material.

Trichochrysea inaequalis Pic (Fig. 17b)

Trichochrysea inaequalis Pic, 1927, Mel. Exot. Entomol. 49: 22 (Tonkin:Chapa; PARIS).

DisTRIBUTION : Laos, Vietnam.

Material examined. LAOS: Tonpheng, 1 ex., 31. iii. 1966, Native collr (BISHOP).

Trichochrysea lameyi (Lefèvre)

Heteraspis Iameyi Lef., 1893, Ann. Soc. Entomol. Fr. 62: 123 (Tonkin; PARIS).

Trichochrysea lameyi: Gressitt \& Kimoto, 1961. Pac. Insects Monogr. 1A: 246 (Tonkin).

DisTRIBUTION : Vietnam.

No additional material.
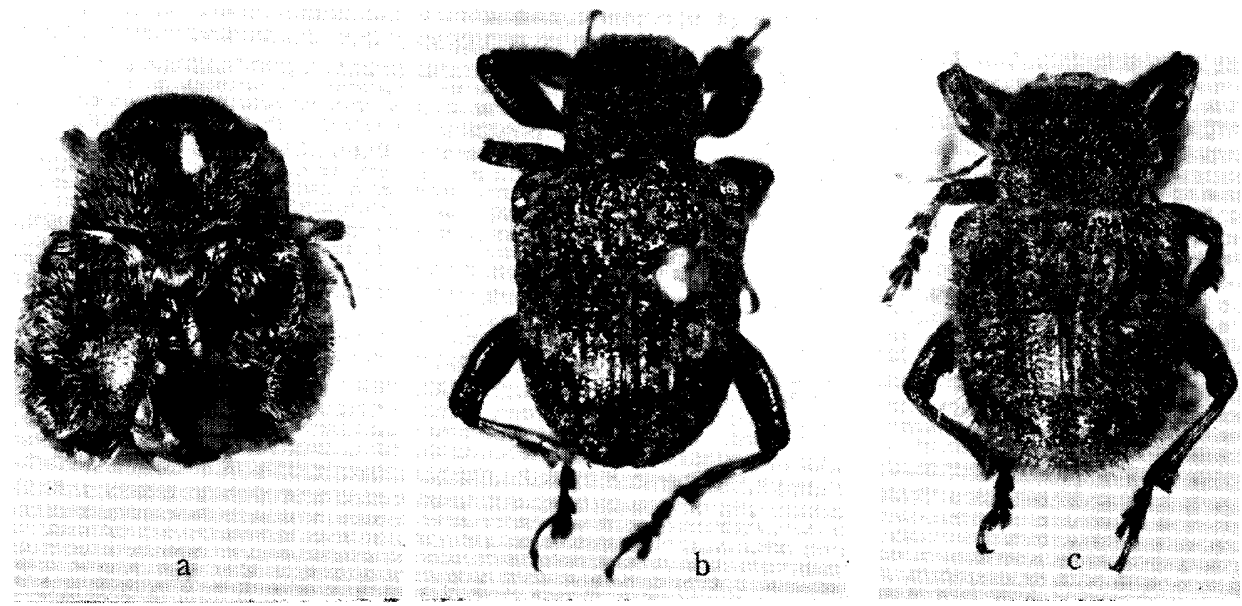

Fig. 17. a, Trichochrysea morosa (Lefèvre); b, T. inaequalis Pic; c, T.robusta Pic. 
Trichochrysea mandarina (Lefèvre)

Heteraspis mandarina Lef ., 1893, Ann. Soc. Entomol. Fr. 62: 125 (Cochinchina; PARIS).

Trichochrysea mandarina : Clavareau, 1914, Coleopt. Cat. Junk 59: 83 (Cochinchina).

Trichochrysea coomani Pic, 1926, Mél. Exot. Entomol. 46: 11 (Tonkin; PARIS). New synonymy.

Distribution : Thailand, Laos, Vietnam.

Material examined. THAildand: Doi Suthep, $100 \mathrm{~m}$, Chiang Mai Prov., 1 ex., 18. vi. 1965, K. Morimoto ; Fang, 3 exs.. 13. v. 1965, K. Morimoto ; Khao Yai Nat. Park, 1 ex., 6. v. 1965, Y. Miyatake ; Mae Chan, 1 ex., 13. vii. 1970, K. Yano (KU). LAOS: Umgeb. Vientiane, 8 exs., iii-vi. 1963; Umgeb. Vanky, 5 exs., 1963, 6 exs., 1964; Umgeb. Pakse, 3 exs., 1963 ; Paklay, 3 exs., 1963 (MUN. CHEN) ; Ban Van Heue, $20 \mathrm{~km}$ E of Phou-kow-kuei, 2 exs., 15-31. v. 1965, Native collr ; Borikhane Prov., Pakkading, 1 ex., 17. v. 1965, 1 ex., 5. iv. 1966, 1 ex., 15. vi. 1966, Native collr ; Sayaboury Prov., Sayaboury, 1 ex., 21. iv. 1966, Native collr (BISHOP). VIETNAM: Kontum, N of Pleiku, 550 m, 1 ex., 13. v. 1960, light trap, R. E. Leech; Ban Me Thuot, 500 m, 1 ex., 16-18. v. 1960, S. Qua te (BISHOP).

Trichochrysea morosa (Lefèvre) (Fig. 17a)

Heteraspis morosa Lef., 1885, Mém. Soc. Sci. Liège, ser. 2, 11: 83, nota 1 (Siam: Juthia ; PARIS) ; 1893, Ann. Soc. Entomol. Fr. 1893: 126 (Bords du Meking et montagnes de Chaudoc).

Trichochrysea morosa: Clavareau, 1914, Coleopt. Cat. Junk 59: 83 (Siam).

Distribution: Thailand, "Indo-China".

Material examined. THAiland : Sam Ngaw Tak, 1 ex., 25. v. 1959 (BANGKHEN).

Trichochrysea mouhoti Baly (Figs. 16c, 18b-c)

Trichochrysea mouhoti Baly, 1860, J. Entomol. 1: 196 (Cambodia ; BM).

Bromius mouhoti: Baly, 1856, J. Entomol. 2: 440 (Cambodia).

Heteraspis mouhoti: Lefèvre, 1893, Ann. Soc. Entomol. Fr. 1893: 123 (Cambodge: LuangPrabang).

Trichochrysea superba Pic, 1934, Mél, Exot. Entomol. 64: 6 (Indo-China; PARIS). N ew synony-

my.

Distribution : Thailand, Cambodia, Laos, Vietnam.

MATERIAL EXAMINED. THAILAND: Bangkok, 1 ex., 22. vi. 1959, Sam Ngow Tak, 1 ex., 26 v. 1959 (BANGKHEM). LAOS: Umgeb. Pakse, 28 exs., 1963; Umgeb. Vientiane, 21 exs., iii-vi. 1963; Umgeb. Paklay, 2 exs., 1963; Umgeb. Vanky, 1 ex., 1964 (MUNCHEN) ; Sedone Prov., Pakse, 1 ex., 16. v. 1965, Native collr, 1 ex., 15. v. 1965, A. D. Ashlock; $5 \mathrm{~km} \mathrm{E} \mathrm{of} \mathrm{Pakse,} 1$ ex., 13. v. 1955, P. D. Ashlock; Sayaboury Prov., Sayaboury, 1 ex., 20. v. 1966, Native collr (BISHOP). VIETNAM: Ban Me Thuot, 500 m, 1 ex., 16 18. v. 1960, R. E. Leech (BISHOP). 


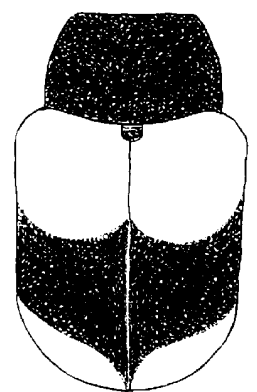

a

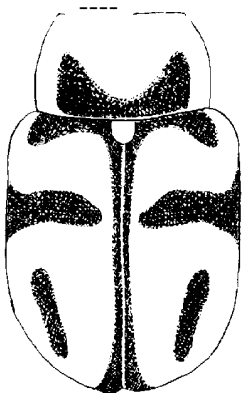

b

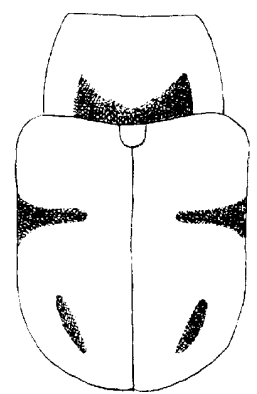

C

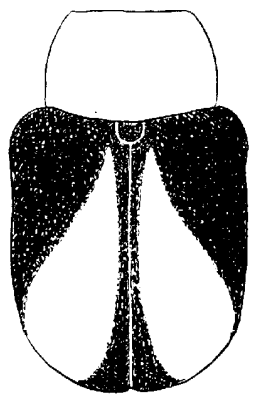

$\mathrm{d}$

Fig. 18. a, Tvichochrysea hcbe (Baly); b-c, T. mouhoti Baly; d, T. vitalisi (Berlioz).

Trichochrysea multicolor Pic

Trichochrysea multicolor Pic, 1926, Mél. Exot. Entomol. 46: 12 (Tonkin; PARIS).

Distribution : Thailand, Laos, Vietnam.

In the type specimen, pronotum is green and elytron is cupreous. The specimens here treated are very variable on the coloration of the dorsal surface.

Material ExAmined. THAILAND: Doi Suthep, $1000 \mathrm{~m}$, Chiang Mai Prov., 3 exs., 18. vi. 1965, K. Morimoto (KU). LAOS: Umgeb. Vientiane, 1 ex., iii-vi. 1963, Umgeb. Pakse, 1 ex., 1963; Umgeb. Vanky, 1 ex., 1964 (MUNCHEN) ; Vientiane Prov., Phou Kou Khouei, 1 ex., 16. vi. 1966, Native collr ; Ban Van Eue, 1 ex., 15-31. v. 1965, Native collr (BISHOP). VIETNAM: $20 \mathrm{~km} \mathrm{~N}$ of Pleiku, 1 ex., 650 m, 9. v. 1960, S. Quate (BISHOP) ; Hoa-Binh, Tonkin, 2 exs., A. Cooman (FREY).

\section{Trichochrysea robusta Pic (Fig. 17c)}

Tuichochrysea robusta Pic, 1926, Mél. Exot. Entomol. 46:12 (Tonkin; PARIS).

Trichochrysea griseonotata Pic, 1936, Mél. Exot. Entomol. 67: 14 (Hoa-Binh ; PARIS), New synonymy.

Trichochrysea fasciataCheII, 1940, Sinensia 11: 506 (Tonkin: Hoa-Binh). New synonymy.

We have not studied the type of fasciata Chen, which was described by a single specimen from Hoa-Binh, collected by A. de Cooman. The type series of griseonotata Pic seems to be same as that of fasciata Chen.

Material Examined. LAOS: Sayaboury Prov., Sayaboury, 1 ex., 5. v. 1966, 1 ex., 20. v. 1966, Native collr (BISHOP). VIETNAM: Hoa-Binh, Tonkin, 7 exs., A. Cooman (FREY).

\section{Trichochrysea similis Chen}

Trichochrysea similis Chen, 1935, Sinensia 6 (3) : 342 (S. China). -Gressitt \& Kimoto, 1961, Pac. Insects Monogr. 1A: 248 (S. China, Tonkin). 
Distribution: S. China, Vietnam.

No additional material.

\section{Trichochrysea tarsata Achard}

Trichochrysea tarsata Achard, 1921, Bull. Soc. Entomol. Fr. 1921: 171 (China). Chen, 1935, Sinensia 6 (3) : 341, fig. 29, 30 (China). Gressitt\& Kimoto, 1961, Pac. Insects Monogr. 1A: 248 (Tonkin).

Distribution : S. China, Vietnam.

MAterial EXAmined. LAOS: Sayaboury Prov., Sayaboury, 1 ex., 17. v. 1965, Native collr (BISHOP). VIETNAM: Hoa-Binh, 4 exs., A. Cooman (FREY).

Trichochrysea vitalisi (Berlioz) New combination (Fig. 18d)

Heteraspis vitalisi Berl 1917, Ann. Soc. Entomol. Fr. 86: 310 (Laos; PARIS).

Distribution : "Thailand, Laos.

Material ExAmined. THAILAND : Sam Ngow Tak, 1 ex., 29. v. 1959 (BANGKHEN). LAOS: Umg. Vientiane, 11 exs., iii-vi. 1963 (MUNCHEN).

\section{Genus Piomera Baly}

Piomera Baly, 1863, J. Entomol. 2:156 (type : Piomera brachialis Baly, from Borneo). --Chapuis, 1874, Genera Coleopt. 10: 278. -Lefèvre, 1885, Mém. Soc. Sci. Liège, ser. 2, 11: 79.

\section{Key to SPECIES of Piomera}

1 (0). Tibia of anterior leg nearly straight and with a minute tooth subapically or without any distinct tooth

Tibia of anterior leg strongly curved and with a large angulation subapically; length $5 \mathrm{~mm}$ (Bryant, 1941, Malaya, Singapore ; Fig. 19c)….... malayana

2(1). Body length shorter; elytron with interstics of longitudinal punctate striae distinctly raised and costate; tibia of anterior leg with a minute tooth subapically; length $3.4-3.5 \mathrm{~mm}$

Body length longer; elytron with interstices of longitudinal punctate striae flat, not costate; tibia of anterior leg without any distinct tooth; length $4.0-4.5 \mathrm{~mm}$

Piomera costata Kimoto \& Greshitt, n. sp. (Fig. 19a)

Body narrowly elongate, subparallel-sided; entirely clothed with adpressed scale like setae white or reddish brown; general color reddish brown, antenna with apical segments in part infuscate.

Head rugosely punctate, vertex depressed on interocular area; clypeus depressed, strongly punctate, not covered by scale like setae, widened anteriorly, emarginate at anterior margin. Antenna slender, filiform, nearly $2 / 3$ as long as body length, finely pubescent ; first segment robust, club-shaped; second nearly $3 / 5$ as long as first, robust; third nearly twice as long as se- 
cond, slender; fourth to sixth subequal to third in length and shape; seventh slightly longer than sixth and gradually widened apically; eighth slightly shorter and robuster than seventh; ninth and tenth subequal to eighth in length and shape; eleventh slightly longer than tenth and its apex pointed. Pronotum subcylindrical, slightly wider than long, side distinctly rounded, strongly rugosopunctate. Scutellum elongate, slightly narrowed posteriorly, apex rounded. Elytron elongate, much wider than pronotum at base, subparallel-sided and rounded at apex, dorsal surface closely punctate and partly arranged in ill-defined longitudinal rows and interstices of punctate striae distinctly raised and costate, especially on latero-apical part. Tibia of anterior leg with a minute tooth subapically.

Length 3.4-3.5 mm.

Holotype (BISHOP), THAILAND: Trang Prov., Khaophappha Khaochang, ZOO-400 m, 10. i. 1964, G. A. Samuelson. Paratopotype: 1 ex., same data as the holotype (KIMOTO) .

This new species somewhat resembles P.major Kimoto \& Gressitt, but differs in being the body length shorter and having the interstices of longitudinal punctate striae distinctly raised and costate and tibia of anterior leg with a minute tooth subapically.

Piomela major Kimoto \& Gressitt, n. sp. (Fig. 19b)

Body narrowly elongate, subparallel-sided ; entirely clothed with adpressed scale like setae white or pitchy brown; general color pitchy brown, clypeus and labrum reddish brown; antenna reddish brown with three or four apical segments infuscate; legs pitchy brown.

Head rugosely punctate, with a longitudinal impressed line on middle, de-
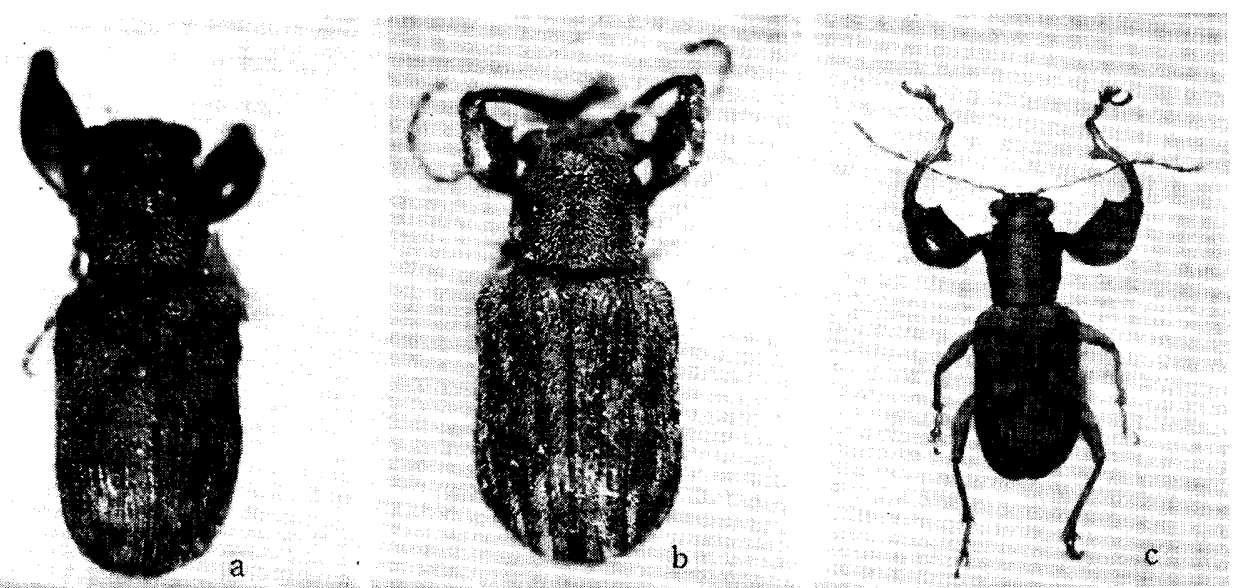

Fig. 19. a, Piomela costata Kimoto \& Gressitt, n. sp .; b, P. major Kimoto \& Gressitt, n. sp.; c, P. malayuna Bryant (1941), from Malaya. 
pressed on inter-ocular area ; clypeus depressed, strongly punctate, not covered by scale like setae and widened anteriorly, emarginate at anterior margin. Antenna slender, filiform, nearly $2 / 3$ as long as body length, finely pubescent; first segment robust, club-shaped; second nearly $2 / 3$ as long as third, robust; third nearly $13 / 4$ times as long as second, slender; fourth to sixth subequal to third in length and shape; seventh slightly longer than sixth and gradually widened apically; eighth nearly $4 / 5$ as long as seventh and much robuster ; ninth nearly as long as eighth but slightly slenderer; tenth subequal to

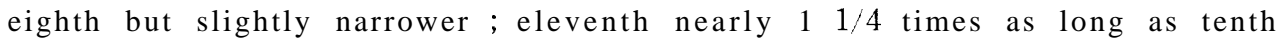
and its apex pointed. Pronotum subcylindrical, transverse, nearly $11 / 3$ times as wide as long; side distinctly rounded, strongly rugoso-punctate. Scutellum elongate, slightly narrowed posteriorly, apex rounded. Elytron elongate, much wider than pronotum at base, subparallel-sided and rounded at apex, dorsal surface closely punctate and partly arranged in ill-defined longitudinal rows and interstices of punctate striae flat.

Length 4.0 -- $4.5 \mathrm{~mm}$.

Holotype (Type No. 2356, Kyushu Univ.), THAILAND: Doi Suthep, 1000 m, Chiang Mai Prov., 12. vi. 1965, K. Morimoto. Paratopotype: 1 ex., same data as the holotype (BISHOP).

This new species somewhat resembles P.malayana Bryant, but differs in having the tibia of anterior leg nearly straight and without any distinct tooth subapically.

\section{Genus Malegia Lefevre}

Malegia Lef., 1883, Bull. Soc. Entomol. Fr. ser. 6, 3: 65; 1885, Mém. Soc. Sci. Liège, ser. 2, 11 : 73 (type : Malegia letourneuxi Lefevre, from Egypt), -Gressitt\& Kimoto, 1961, Pac. Insects Monogr. 1A: 266.

Microlypesthes: Chen, 1940, Sinensia 11(5-6): 491.

\section{Key to SPecies of Malegia}

Legs entirely reddish brown; cupreous with slight greenish luster; antenna pitchy

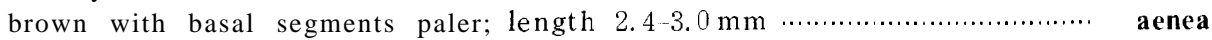

Legs pitchy black with anterior and middle tibiae reddish brown; blackish, antenna with basal segments paler; length $2.4-3.0 \mathrm{~mm}$

bicoloripes

\section{Malegia aenea(Chen) New combination (Fig. 20a)}

Microlypesthes aeneus Chen, 1940, Sinensia 11 (5-6) : 517 (China).

Malegia flavipes Chûjô, 1942, Mushi, Fukuoka 14 (1) :55 (Kwangtung ;TARI). Gressitt \& Kimoto, 1961. Pac. Insects Monogr. 1A: 266 (China). New synonymy.

Distribution: China, Vietnam, Thailand.

Material examined. THAILAND: Chiangmai Prov.. Chiangmai, 2 exs., 9. vi. 1965, P. D. Ashlock; Mae Cho, Chiangmai Prov., 1 ex., 9. vi. 1965, P. D. 
Ashlock (BISHOP). VIETNAM: Ninh Hoa, N of Nha Trang, 1 ex., 28. xi. 1960, C. M. Yoshimoto; Nha Trang, 1 ex., 17-26. xi. 1960, C. M. Yoshimoto (BISHOP).

Malegia bicoloripes $\mathrm{Pic}$

Malegia bicoloripes Pic, 1936, Mél.Exot. Entomol. 67: 16 (Tonkin ; PARIS).

Distribution : Vietnam.

No additional material.

\section{Genus Lypesthes Baly}

Lypesthes Baly, 1863, J. Entomol. 2: 152 (type : Fidia atra Motschulsky, from Japan). Chapuis, 1874, Genera Coleopt. 10: 273. Lefèvre, 1885, Mém. Soc. Sci. Liège, ser. 2, 11: 74. Jacohy, 1908, Fauna India, Coleopt. 2: 412. Chen, 1935, Sinensia 6 (3) : 370; 1940, ibid. 11 (5-6) : 491. - Chû jô, 1956, Philip. J. Sci. 85 (1) : 101. Gressitt \& Kimoto, 1961, Pac. Insects Monogr. 1A:269. Ohno, 1958, J. Toyo Univ. 12: $178 . \quad$ Kimoto, 1964, J. Fac. Agr. Kyushu Univ. 13 (2) : 256.

Leprotes Baly, 1863, J. Entomol. 2: 158 (type : Endoxus gracilicornis Baly, from Hongkong). - Chapuis, 1847, Genera Coleopt. 10: 279. Lefèvre, 1885, Mkm. Soc. Sci. Liège, ser. 2, $11: 80$. - Jacoby, 1908, Fauna India, Coleopt. 2: 425.

Talmonus Fairmaire, 1889, Ann. Soc. Entomol. Fr. ser. 6, 9: 71 (type: Talmonus farinosus Fairmaire, from Moupin).

\section{KEY TO SPECIES OF Lypesthes}

1(0). Elytron covered with adpressed and erect hairs, sometimes with white

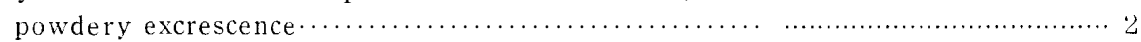

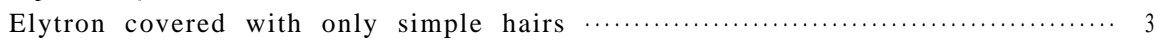

2 (1). Median longitudinal area of pronotum, a broad longitudinal stripe parallel to elytral suture from base to apex, two other short but equally broad longitudinal stripes at base, and a broad transverse band on middle portion of each elytron, covered by chestnut hairs, and posterior portion covered by pale yellow hairs; length $7 \mathrm{~mm} . . . .2 . \ldots \ldots$ bisquamosus

Dorsal surfaces covered by adpressed extremely fine white hairs and erect white hairs, and also covered with white powdery excrescence;

black, antenna with basal segments brownish; length $3.4-5.0 \mathrm{~mm}$........ albidus

3 ( 1 ). Dorsal surfaces covered with extremely fine white hairs; black, antenna with basal segments brownish; length $8 \mathrm{~mm} \ldots \ldots \ldots \ldots \ldots \ldots \ldots$ impressus

Dorsal surfaces thickly covered by stout hairs and not covered with white powdery excrescence; coloration reddish brown; length $3.0 \mathrm{~mm} \cdots$ rufus

Lypesthes albidus Pic (Fig. 20b)

Lypesthes albidus Pic, 1923, Mél. Exot. Entomol. 40: 16 (Cochinchina; PARIS).

Distribution : Thailand, Laos, Vietnam.

Material examined. THAILAND: Chiang Mai Prov., Tan Keo, Doi Suthep, 1 ex., 10. vi. 1965, Y. Miyatake (KU). LAOS: 100 m, Uekinak, nr Pakkading, 1 ex., 22. iv. 1965, J. L. Gressitt (BISHOP). VIETNAM: $7 \mathrm{~km}$ SE of Dilinh 


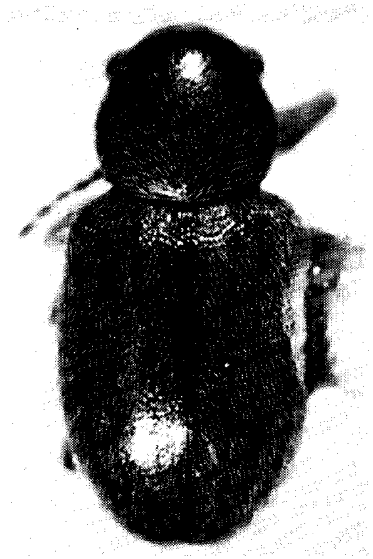

a

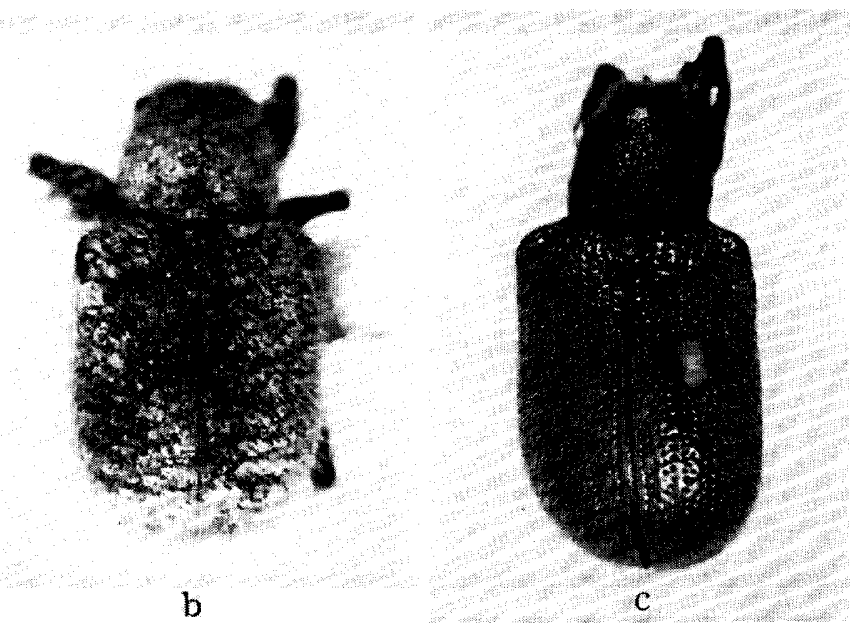

Fig. 20. a, Malegiaaenea (Chen); b, Lypesthes albidus Pic;c, L. fulvus Baly (1878), from

S. China, Taiwan, Japan.

(Djiring), $990 \mathrm{~m}, 1$ ex., 2. v. 1960, R. E. Leech; $20 \mathrm{~km} \mathrm{~N}$ of Peliku, $650 \mathrm{~m}, 1$ ex., 9. v. 1960, L. W. Quate (BISHOP).

\section{Lypesthes bisquamosus Chen}

Lypesthesbisquamosus Ch e n, 1935. Sinensia $6: 374$ (Tonkin; PARIS). --Gressitt \& Kimoto, 1961, Pac. Insects Monogr. 1A: 270 (Tonkin).

Distribution : Vietnam.

No additional material.

\section{Lypesthes impressus (Pic) New combination}

Leprotes impressaPic, 1928, Mél. Exot. Entomol 52: 26 (Tonkin:Chapa; PARIS).

Distribution: Laos, Vietnam.

Material Examined. LAOS: Sayaboury Prov., Sayaboury, 1 ex., 5. v. 1966, Naitve collr (BISHOP). VIETNAM: Hoa-Binh, Tonkin, 3 exs., A. Cooman (FREY).

\section{Lypesthes rufus (Pic) New combination}

Leprotes rufus Pic, 1924, Mél. Exot. Entomol. 41: 6 (Tonkin).

Distribution: Vietnam.

We could not trace the type location of this species. Judging from the original description, it is doutful whether this species is surely a member of present genus. Pic compared this species with Lypesthes fulvus (Baly) (Fig. 20c). The body length of this species is $3 \mathrm{~mm}$ and of fulvus is $7 \mathrm{~mm}$ or so. 


\section{Genus Pseudometaxis Jacoby}

Pseudometaxis Jac., 1900, Mém.Soc. Entomol. Belg. 7: 101 (type : Pseudometaxis serratithorax Chen, =Pseudometaxis serraticollis Jacoby, 1900, from Burma, nec Baly, 1867, from Penang) ; 1908, Fauna India, Coleopt. 2: 424. Pic, 1929, Bull Soc. 2001. Fr. 54: 140. -Chen, 1935, Sinensia 6 (3) : 347; 1940, ibid. 11 (5-6) : 490. -Chûjô, 1956, Philip. J. Sci. 85 (1) : 88. --Gressitt \& Kimoto, 1961, Pac. Insects Monogr. 1A: 253.

\section{Key to species of Pseudometaxis}

$1(0)$. Suberect hairs covering dorsal surface of elytron closer, longer and slenderer; pitchy brown, in some specimens femora brownish in various degrees; antenna reddish brown with apical segments brownish ............. 2

Suberect hairs covering dorsal surface of elytron sparser, shorter and robuster; adpressed scales covering dorsal surface of elytron partly brownish and partly pitchy brown, and in many cases forming irregular bands; reddish to pitchy brown, antenna and legs reddish brown ;

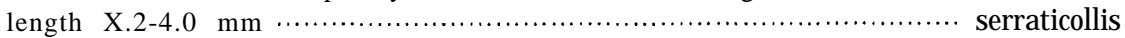

2(1). Dorsal surface of elytron without any distinct markings or bands; length

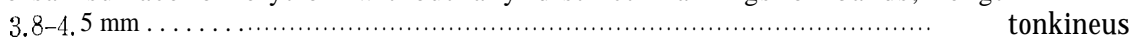
Dorsal surface of elytron with distinct marking or band; length 3.24. $2 \mathrm{~mm}$ minutus

Pseudometaxis minutus Pic (Fig;. 21a)

Pseudometaxis minutus Pic, 1923, Mél. Exot. Entomol. 40: 16 (China ; PARIS) ; 1929, Bull. Soc. Zool. Fr. 54: 141 (China, Tonkin).-Chen, 1935, Sinensia 6 (3) : 348 (Yunnan). -- Gressitt \& Kimoto, 1961, Pac. Insects Monogr. 1A: 254 (Yunnan).

Leprotes submaculatus Pic, 1924, Mél. Exot. Entomol. 41: 6(Tonkin; PARIS). New synonymy. Pseudometaxis minutus var. angulosus Pic, 1929, Bull. Soc. Entomol. Fr. 1929: 147 (Yunnan).

Pseudometaxis bicoioripes Pic, 1929, Bull. Soc.Zool. Fr. 54: 141 (Yunnan; with var. reductus; PARIS). -Gressitt \& Kimoto, 1961, Pac. Insects Monogr. 1A: 254 (=minutus).

Distribution: SW China, Vietnam, Thailand.

Material ExAmined. THAILAND: Doi Suthep, 1300 m, Chiang Mai Prov., 1 ex., 8. vi. 1965, P. D. Ashlock (BISHOP), Doi Suthep, 1000 m, 1 ex., 12. v. 1965, 1 ex., 18. vi. 1965, K. Morimoto ;Doi Pui, 1300 m, 1 ex., 8. vi. 1965, Y. Miyatake (KU). VIETNAM: Hoa-Binh, Tonkin, 1 ex., A. Cooman (FREY).

\section{Pseudometaxis serraticollis (Baly) (Fig. 21b)}

Demotina serraticollis Baly, 1867, Trans. Entomol. Soc. Lond., ser. 3, 4 (2) : 85 (Penang). -Jacoby, 1889, Ann. Mus. Civ. Genova 27: 169 (Burma). -Lefèvre, 1893, Ann. Soc. Entomol. Fr. 122 (Cochinchina, Sumatra). -Jacoby, 1908, Fauna India, Coleopt. 2: 429 (Burma, Penang).

Pseudometaxis serraticollis: Chen, 1940, Sinensia 11 (5-6): 509 (genus).

Pseudometaxis maculatus Chûjô, 1964, Nature and Life in SE Asia, Kyoto 3: 279. pl. 25, fig. 16 (Thailand ; CHUJO). New synonymy.

Distribution: Burma, Thailand, Vietnam, Malaya, Sumatra.

We could not trace the type location of serraticollis.

Material examined. THAilAND: Chiang Mai Prov., Doi Suthep, $1300 \mathrm{~m}$, 

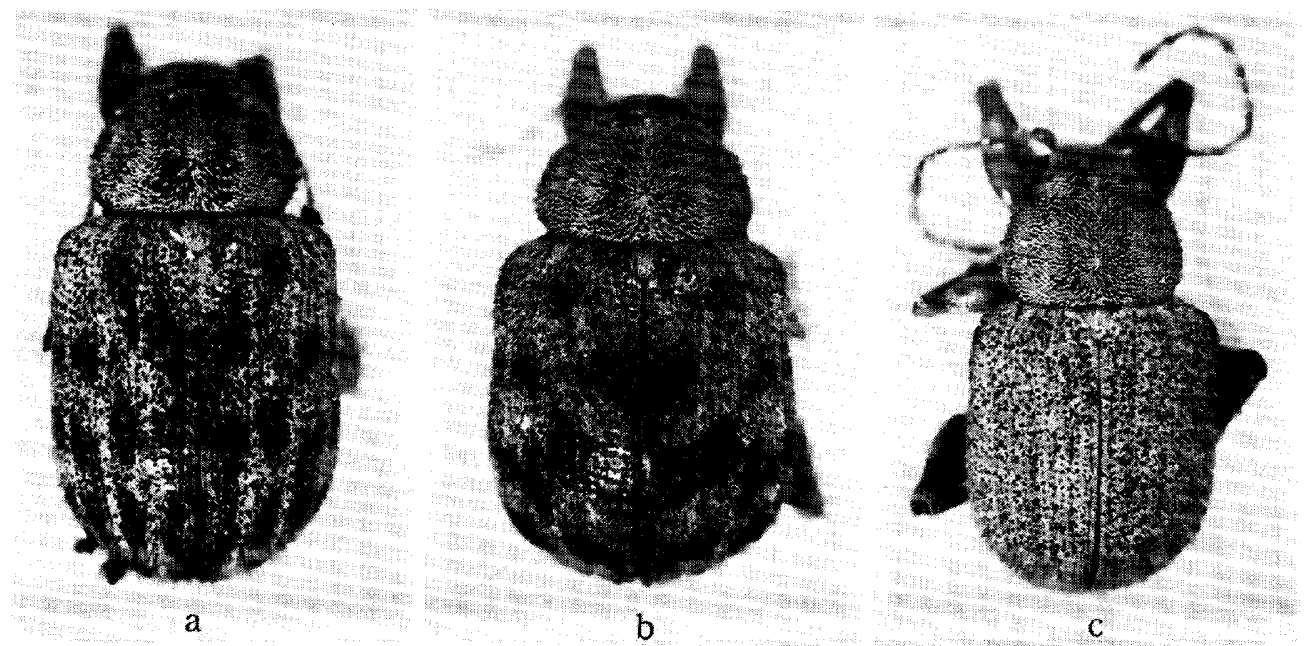

Fig. 21. a, Pseudometaxis minutus $\mathrm{Pic} ; \mathrm{b}$, P. serraticollis (I3aly); c, P. tonkineus Pic.

2 exs., 8. vi. 1965, P. D. Ashlock (BISHOP) ;Doi Suthep, 1000 m, 1 ex., 17. vi. 1965, K. Morimoto; Doi Pui, 1300 m, 2 exs., 17. vi. 1965, K. Morimoto, Doi Pui, 1685 m, 2 exs., 17. vi. 1965, Y. Miyatake; Botanical Garden, Chiang Mai, 1 ex., 12. vi. 1965, Y. Miyatake; Chiang Mai, 1 ex., 12. vi. 1965, K. Morimoto; Mae Klang Water Fall, nr. Chom Thong, 33 exs., 11. vi. 1965, K. Morimoto \& Y. Miyatake; Khao Chong, nr. Trung, 1 ex., 26. vi. 1965, Y. Miyatake (KU).

\section{Pseudometaxis tonkineus Pic (Fig. 21c)}

Pseudometaxis tonkineus Pic, 1924, Mél.Exot. Entomol. 41 : 7 (Tonkin; PARIS) ; 1929, Bull. Soc. 2001. Fr. 54: 141 (Tonkin).

Pseudometaxis hirsutus Pic, 1928, Mél.Exot. Entomol. 52: 26 (Tonkin: Chapa; PARIS) ; 1929, Bull. Soc.Zool. Fr. 54: 141 (Tonkin). New synonymy.

Distribution: Thailand, Vietnam.

This species closely resembles P.minutus Pic, but is separable in having the dorsal surface of elytron without any distinct marking. However, it is possible that this species is nothing but a infraspecific variation of minutus Pic.

Material examined. THAiland: Chiang Mai Prov., Doi Suthep, 1000 m, 1 ex., 12. vi. 1965, P. D. Ashlock (BISHOP), 1 ex., 8. vi. 1965, K. Morimoto, 5 exs., 18. vi. 1965, K. Morimoto \& Y. Miyatake; Doi Pui, Chiang Mai Prov., 3 exs., 8. vi. 1965, Y. Miyatake (KU).

\section{Genus Hyperaxis Gemminger \& Harold}

Metaxis Baly, 1863 (ncc de Chaudoir, 1850), J. Entomol. 2: 157 (type : Metaxis sellata Baly, from Borneo). Chapuis, 1874, Genera Coleopt. 9: 279.

Hyperaxis Gemm. \& Har,, 1874, Cat. Coleopt.9: 3377. Lefèvre, 1885, Mém. Soc. Sci. Liège, ser, 2, 11: 79. Jacoby, 1908, Fauna India, Coleopt. 2: 417. -Chen, 1940, Sinensia 11 
(5-6) : 490. Gressitt \& Kimoto, 1961, Pac. Insects Monogr. 1A : 252. Kimoto, 1964, J.

Fac. Agr. Kyushu Univ. 13(2): 253.

\section{K EY TO SPECIES of Hyperaxis}

1 (0). Dorsal surface of elytron covered with erect hairs and adpressed scales . 2 Dorsal surface of elytron covered with adpressed scales only; pale to dark reddish brown, antenna entirely reddish brown; length $5.0 \mathrm{~mm} \ldots .$. scutellatus

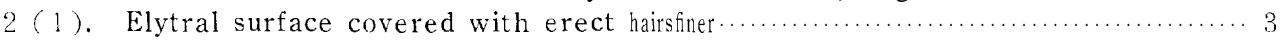

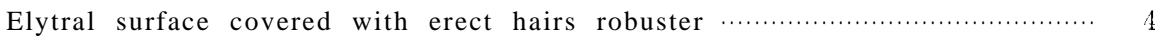

3 (2). Elytral surface with some irregular markings blackish, erect hairs longer and adpressed scales finer; yellowish to reddish brown ; length 3. $0-4.2$

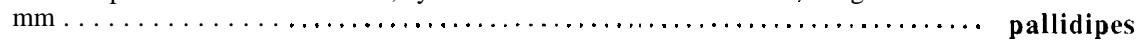

Elytral surface without any distinct markings, erect hairs shorter, and adpressed scales robuster; yellowish to reddish brown, with tibiae, tarsi and apex of femora pitchy brown; length $3.8-4.0 \mathrm{~mm} \ldots \ldots \ldots \ldots \ldots$ tanongchiti

4 (2). Elytral surface without any distinct markings; black, antenna reddish brown with apical segments pitchy brown; legs black with median portion of femora of middle leg brownish ; length 4.2 $5.2 \mathrm{~mm} \ldots \ldots \ldots \ldots \ldots \ldots$ ariyani

Elytral surface with several stripes or markings white; black, antenna dark reddish brown with apical segments slightly darker; legs mostly black; length $1.24 .5 \mathrm{~mm} \ldots \ldots \ldots \ldots \ldots \ldots \ldots \ldots \ldots \ldots \ldots \ldots \ldots$ maculat

Hyperaxis ariyani Chûjồ (Fig. 22a)

Hyperaxis ariyani Chûjồ, 1964, Nature and Life in SE Asia, Kyoto 3:273, pl. 25, fig. 15 (Thailand : Chieng Mai;CHUJO).

Distribution : Thailand.

Material examined. Thailand: Chiangmai Prov., Doi Pui, 2 exs., 2. iv. 1958, 5 exs., 1 8. iv. 1958, T. C. Maa (BISHOP), 3 exs., 8. vi. 1965, Y. Miyatake (KU).

Hyperaxis maculatus Kimoto \& Gressitt, n. sp. (Fig. 22b)

Oval; general color black, elytral surface with several stripes or markings white; antenna dark reddish brown with apical segments slightly darker; legs largely pitchy black.

Head thickly covered with scale like setae, with a feeble longitudinal impressed line on middle and depressed on interocular area ; clypeus convex, not covered with scale like setae, strongly punctate, widened anteriorly. Antenna slender, filiform, nearly as long as body length; first segment robust, somewhat club-shaped; second nearly $2 / 3$ as long as first, and $11 / 3$ times as long as wide; third slender, nearly $23 / 4$ times as long as second, slender; fourth to seventh subequal to third in length and shape; eighth slightly shorter than seventh; ninth to tenth subequal to eighth in length and shape; eleventh subequal to tenth in length but its apex pointed. Pronotum transverse, nearly $11 / 3$ times as wide as long, side rounded, widest slightly behind middle 
and strongly narrowed anteriorly and less strongly so posteriorly; dorsum strongly convex, closely covered with scale like setae. Scutellum subpentagonal, narrowed posteriorly and apex rounded; dorsum thickly covered by scale like setae. Elytron much wider than pronotum at base, slightly widened posteriorly and rounded at apex; dorsum strongly convex, thickly covered with erect hairs black and adpressed scales black and white, and forming several stripes or markings. Anterior and posterior femora each with a large tooth.

Length $4.2-4.5 \mathrm{~mm}$.

Holotype (BISHOP), VIETNAM: Ban Me Thout, $500 \mathrm{~m}, 1$ ex., 16-18. v. 1960, S. Quate. Paratypes : VIETNAM: $33 \mathrm{~km} \mathrm{NE}$ of Ban Me Thuot, $870 \mathrm{~m}, 1$ ex., 18. v. 1960, L. W. Quate (KIMOTO) ; $7 \mathrm{~km} \mathrm{SE} \mathrm{of} \mathrm{Dilinh} \mathrm{(Djiring),} 990 \mathrm{~m}, 1$ ex., v. 1960, R. E. Leech (BISHOP) ; LAOS: Wapikhamthong Prov., Wapi, 1 ex., 15. iii. 1967, light trap, Native collr (BISHOP).

This new species closely resembles $H$. scutellatus (Baly), but differs in having the robust erect hairs beside the adpressed scales on the surface of elytron.

Hyperaxis pallidipes (Pic) New combination (Fig. 24a)

Pseudometaxis pallidipes Pic, 1929, Bull. Soc. Entomol. Fr. 54: 147 (Indo-China; PARIS) ; 1929, Bull. Soc. 2001. Fr. 54: 141 (Tonkin).

Distribution : Thailand, Vietnam.

Material examined. Thailand: Chiang Mai Prov., Doi Pui, $1685 \mathrm{~m}, 1$ ex., 17. vi. 1965, P. D. Ashlock (BISHOP), Doi Pui, 1300-1685 m, 2 exs., 17. vi. 1965, K. Morimoto \& Y. Miyatake (KU). VIETNAM: Dak Song, $75 \mathrm{~km} \mathrm{SW}$ of Ban Me Thuot, 870 m, 1 ex., 19-21. v. 1960, L. W. Quate ; DiLinh (Djiring), 1200 m, 2 exs., 22-28. iv. 1960, L. W. Quate ; Pleiku, 700 m, 1 ex., 8-14. v. 1960, Malaise trap, L. W. Quate; $20 \mathrm{~km} \mathrm{~N}$ of Pleiku, $650 \mathrm{~m}, 2$ exs., 9. v. 1960, S. Quate (BISHOP).

\section{Hyperaxis scutellatus (Baly)}

Demotina scutellata Baly, 1863, J. Entomol. 2: 159 (N. China; BM).

Hyperaxis harmandi Lefèvre, 1893, Ann. Soc. Entomol. Fr. 62: 122 (Tonkin; PARIS). New synonymy.

Pseudometaxis inhirsutus Pic, 1923, Mel. Exot. Entomol. 40: 16 (China ; PARIS) ; 1929, Bull. Soc. 2001. Soc. Fr, 54: 140 (China). Gressitt \& Kimoto, 1961, Pac. Insects Monogr. $1 \mathrm{~A}: 253$ (= scutellatus).

Leprotes rufonotatus Pic, 1923, Mel. Exot. Entomol. 41 :6(Tonkin; PARIS). New synonymy.

Pseudometaxis robustus Pic, 1929, Bull. Soc. 2001. Fr. 54: 140 (Tonkin:Chapa; PARIS). New synonymy.

Pseudometaxis niger Chen, 1935, Sinensia 6 (3) : 348, fig. 31, 32 (China ; PARIS). -Gressitt \& Kimoto, 1961, Pac. Insects Monogr. 1A:253 (=scutellatus).

Hyperaxis niger: Chen, 1940, Sinensia 11 (5-6) : 509 (China).

Hyperaxis scutellatus: Gressitt \& Kimoto, 1961, Pac. Insects Monogr. 1A: 253 (China). Distribution: Vietnam, Hainan, S. China. 

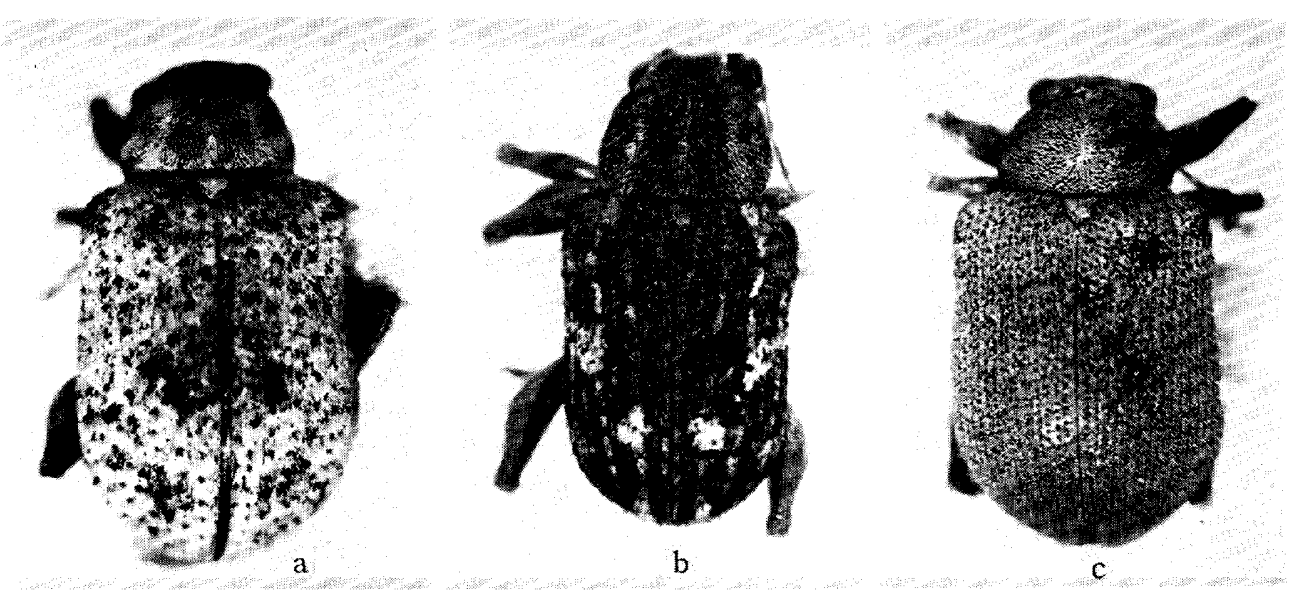

Fig. 22. a, Hyperaxis ariyai Chûjô; b, H. maculatus Kimoto \& Gressitt, n. sp.; c, $H$. tanongchiti Kimoto \& Gressitt, n. sp.

The type of L.rufonotatus Pic, preserved in Paris Museum, is not in good condition. However, this species seems to be the same as scutellatus (Baly). The type of Hyperaxis harmandi Lefèvre is the most pale colored specitnen and of P.robustus is the most dark colored one.

No additional material.

Hyperaxis tanongchiti Kimoto \& Gressitt, n. sp. (Fig. 22c)

Oval; general color yellowish to reddish brown; antenna reddish brown with four or five apical segments pitchy brown; legs reddish brown with tibiae, tarsi and apex of femora pitchy brown.

Head thickly covered with scale like setae, with a feeble longitudinal impressed line on middle and depressed on interocular area; clypeus convex, not covered with scale like setae, strongly punctate, widened anteriorly. Antenna robust, nearly $3 / 4$ as long as body length; first segment robust, somewhat club-shaped; second nearly half as long as first and $11 / 2$ times as long as wide; third slender, nearly $21 / 2$ times as long as second; fourth slightly shorter than third; fifth subequal to fourth in length and shape; sixth slightly shorter than fifth; seventh as long as sixth but widened apically; eighth shorter than seventh and nearly twice as long as wide; ninth and tenth subequal to eighth in length and shape; eleventh slightly longer than tenth and its apex pointed. Pronotum transverse, nearly $13 / 5$ times as wide as long, side rounded, widest slightly behind middle and strongly narrowed anteriorly and less strongly so posteriorly; dorsum strongly convex and slightly depressed along anterior margin, closely covered with scale like setae. Scutellum subpentagonal, narrowed posteriorly and apex rounded, dorsum thickly covered by scale like setae. Elytron much wider than pronotum at 
base, slightly widened posteriorly and rounded at apex; dorsum strongly convex, without any distinct marking, thickly covered with erect hairs and ad. pressed scales both brown. Anterior and posterior femora each with a large tooth.

Length 3.8-4.0 $\mathrm{mm}$.

H olotype (BANGKHEN), THAILAND: Uthaithani, 13. iv. 1963. Paratopotype : 1 ex., same data as the holotype (KU).

This new species somewhat resembles $H$. pallidipes (Pic), but is separable in having the erect hairs shorter and the adpressed scales robuster.

The scientific name of this new species is dedicated to Dr. Tanongchit Wongsiri, Director of Entomology and Zoology Division, Department of Agriculture, Bangkhen, Bangkok.
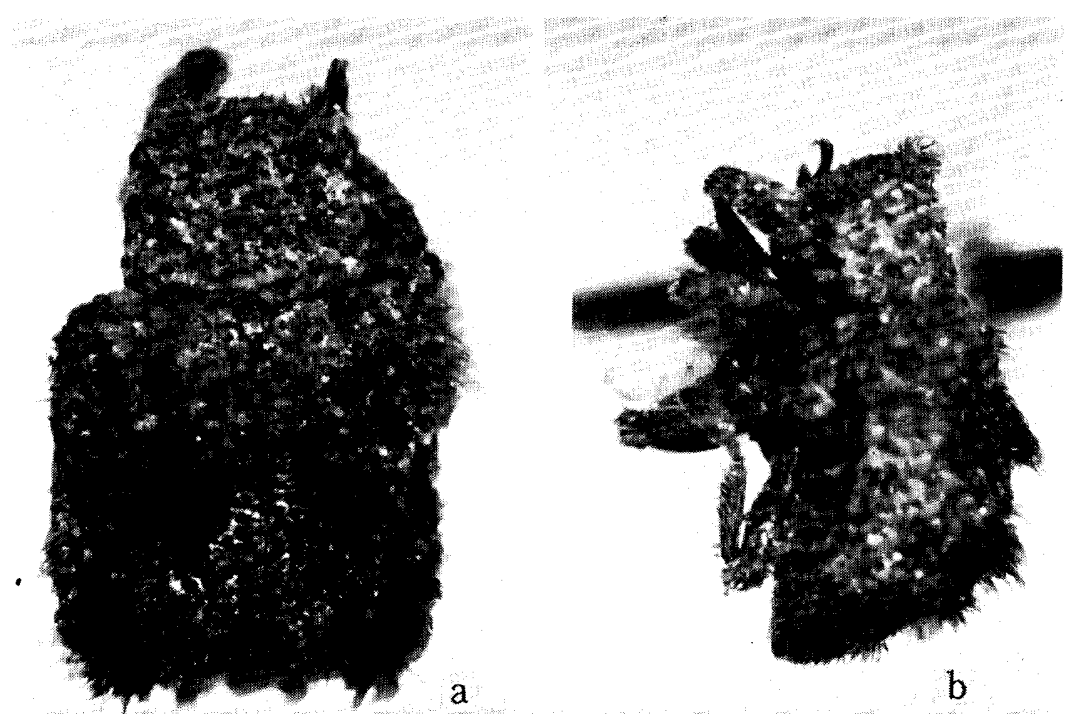

Fig. 23. a, Hemiplatys pascoei Baly, clorsal view, b, ditto, lateral view.

\section{Genus Hemipla tys Baly}

Hemiplatys Baly, 1863, J. Entomol. 2: 160 (type : Hemiplatys pascoei Baly, from Cambodia).

Chapuis, 1874, Genera Coleopt. 10: 282. Lefèvre, 1885, Mém. Soc. Sci. Liège, ser. 3, 11: 81 .

\section{Hemiplatys pascoei Baly (Fig. 23)}

Hemiplatys pascoei Baly, 1863, J. Entomol. 2: 160 (Cambodia; BM).

Distribution : Cambodia, Vietnam.

Body subelongate, subcylindrical, piceous, covered above with long, suberect, strongly curved scales; surface of thorax irregular, remotely punctate, 
border scales on disc intermingled with slender hair-like scales; elytron deeply punctate, interspaces thickened and elevated; disc with five or six tufts of large, rigid, erect, blackish scales, apices of which are, as usual, prolonged into a slender thread; length 3.2-3.6 $\mathrm{mm}$.

Material examined. VietnaM: Mt. Lan Bian, 1500-2000 m, 1 ex., 19. v.8. vi. 1961, N. R. Spencer (BISHOP).

\section{Genus Demotina Baly}

Demotina Baly, 1863, J. Entomol. 2: 158 (type : Demotinabowringii Baly, from Hongkong). -Chapuis, 1874, Genera Coleopt. 10: 281. -Lefèvre, 1885, Mém. Soc. Sci. Liège, ser. 2, 11: 80. -Jacoby, 1908, Fauna India, Coleopt. 2: 427. -Chen, 1935, Sinensia 6 (3): 250; 1940, ibid. 11 (5-6) : 490. Chûjô, 1956, Philip. J. Sci. 85 (1) : 92. -Gressitt \& Kimoto, 1961, Pac. Insects Monogr. 1A:250. --Kimoto, 1964, J. Fac. Agr. Kyushu Univ. 13(2): 254.

\section{Key to species of Demotina}

Each elytron with a longitudinal ridge behind middle and in some cases with another one latero-apically; reddish brown to pitchy black, pronotum and elytron marked with irregular, ill-defined blackish markings ; antenna entirely reddish brown; legs reddish brown with apical portions of femora, subbasal and subapical portions of tibiae blackish ; length $3.3-3.8 \mathrm{~mm} \cdots \cdots \ldots \ldots \ldots \ldots \ldots$ tuberosa

Each elytron without any distinct ridge; dark reddish brown to pitchy brown; antenna reddish brown, with apical segments usually darker, each elytron sometimes with a white marking consisting of mass of scales on subbasal area, and with ill-defined irregular blackish markings which vary in their

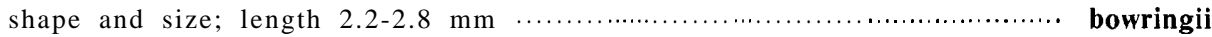

\section{Demotina bowringii Baly (Fig. 24b)}

Demotina bowringii Baly, 1863, J. Entomol. 2 : 159 (Hongkong; BM). --Chen, 1935, Sinensia 6 (3) : 351 (China). -Gressitt \& Kimoto, 1961, Pac. Insects Monogr. 1A: 250 (China).

Distribution : Thailand, Laos, Vietnam, S. China, Hainan.

M aterial examined. Thailand: Doi Pui, Chiang Mai, 1300 m, 2 exs., 17. vi. 1965, K. Morimoto (KU), 1 ex., 2. iv. 1958, T. C. Maa (BISHOP). LAOS: Sedone Prov., Paksong, 3 exs., 17-18. v. 1965, P. D. Ashlock (BISHOP). VIETNAM: Dalat, 1500 m, 4 exs., 29. iv. - 4. v. 1960, L. W. Quate; Dalat, 6 km S, 1400-1500 m, 2 exs., 9. vi. - 7. vii. 1961, N. R. Spencer; $18 \mathrm{~km} \mathrm{NW}$ of Dalat, 1300 m, 2 exs., 4-5. v. 1960, S. Quate \& L. W. Quate ; Fyan, 1200 m, 3 exs., 11. vii. 1. viii. 1961, N. R. Spencer ; Fyan, 900-1000 m, 1 ex., 11. vii. - 9. viii. 1961, N. R, Spencer; Blao (Balao), 5000 m, 2 exs., 14-21. x. 1960, C. M. Yoshimoto; Mt. Lang Bian, 1500-2200 m, 1 ex., 9. v. - 8. vi. 1961, N. R. Spencer ; M'Drak, E of Ban Me Thuot, 4-600 m, 1 ex., 8-19. xii. 1961, N. R. Spencer; Ap Hung-Lam, 21 km NW of Dilinh, 1100 m, 1 ex., 29. ix. - 5. x. 1960, C. M. Yoshimoto (BISHOP). 

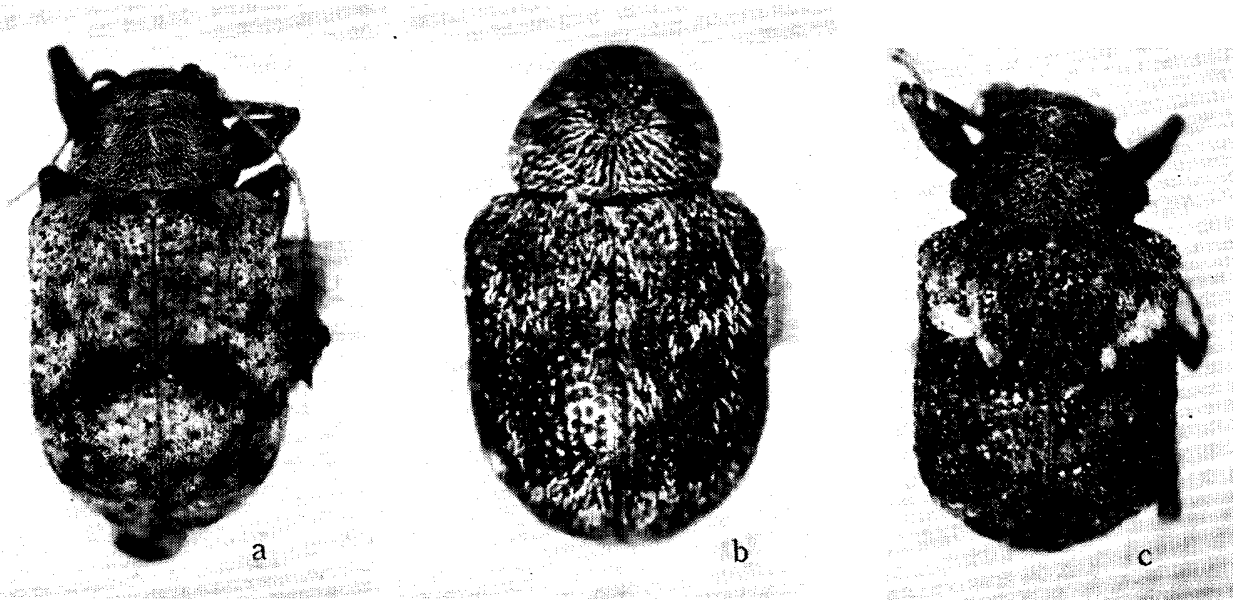

Fig. 24. a, Hyperaxis pallidipes (Pic); b, Demotina bowringii Baly; c, D. tuberosa Chen.

\section{Demotina tuberosa Chen (Fig. 24c)}

Demotinatuberosa C he n, 1935, Sinensia 6 (3) : 354, fig. 33 (S. China, Tonkin ; PARIS). -Gressitt \& Kimoto, 1961, Pac. Insects Monogr. 1A: 252 (S. China, Tonkin).-Kimoto, 1964, J. Fac. Agr. Kyushu Univ. 13 (2): 255 (Japan).

Demotina japana Ohno, 1960, Trans. Shikoku Entomol. Soc. 6 (6) : 65 (Japan ;OHNO). —Chûjô \& Kimoto, 1961, Pac. Insects 3 (1) : 144 (=tuberosa).

Distribution: Laos, Vietnam, S. China, Japan.

Material examined. LAOS: Sedone Prov., Paksong, 2 exs., 17-18. v. 1965, P. D. Ashlock (BISHOP). VIETNAM: Fyan, $1200 \mathrm{~m}, 2$ exs., 11. vii. - 9. viii. 1961, N. R. Spencer; $20 \mathrm{~km}$ from DiLinh (Djiring), 1 ex., 22-28. iv. 1960, L. W. Quate (BISHOP).

\section{Genus Aoria Baly}

Aoria Baly, 1863, J. Entomol. 2: 149 (type : Adoxus nigripes Baly, from Hong Kong). -Chapuis, 1874, Genera Coleopt. 10: 270. -Lefèvre, 1885, Mém. Soc. Sci. Liège, ser. 2, 11: 71. -Jacoby, 1908, Fauna India, Coleopt. 2: 396. - Chen, 1935, Sinensia 6 (3) : 362; 1940, ibid. 11 (5-6) : 491. -Chûjô, 1956, Philip. J. Sci. 85 (1) : 111. -Gressitt \& Kimoto, 1961, Pac. Insects Monogr. 1A: 256. -Kimoto, 1964, J. Fac. Agr. Kyushu Univ. 13 (2) : 260.

Pseudaoriana Pic, 1930, Mél. Exot. Entomol. 56: 3 (type : Pseudaoria (Pseudaoriana) lemoulti Pic,=Aoriarufotestacea Fairmaire).

\section{Key to species of Aoria}

Elytron reddish brown with three pairs of black spots; pronotum reddish brown with or without a blackish marking at middle; reddish brown, ventral sur-

faces in part blackish, antenna reddish brown; length 5. $56.0 \mathrm{~mm} \cdots \cdots \cdots$ bowringii

Elytron without black spots; coloration variable: dorsum entirely reddish brown, entirely black or reddish brown with prothorax black, ventral surfaces 
entirely black, entirely reddish brown or reddish brown with prothorax blackish; antenna blackish with basal segments brownish, legs blackish, in some specimen brownish in various degrees ; length $4.2-5.8 \mathrm{~mm} \ldots \ldots \ldots \ldots \ldots$ nigripes

Aoria bowringii (Baly) (Fig. 25a)

Adoxus bowringii Baly, 1860, J. Entomol. 1: 27 (Nepal, N. India ; BM).

A oria bowringii: Baly, 1867, Trans. Entomol. Soc. Lond., ser. 3, 4 (2) : 79, pl. 4, fig. 1 (Malacca, Borneo, Penang, Burma, Cambodia, N. China). -Lefèvre, 1889, Ann. Soc. Entomol. Fr., ser. 6, 9: 296 (Tonkin: Lien-Son) ; 1890, Nouv. Arch. Mus. Paris, ser. 3, 2: 193 (Laos) ; 1893, Ann. Soc. Entomol. Fr., 1893: 121 (Tonkin: Ha-Lang) ; 1904, Miss. Pavie Indo-China, ser. 3, 3: 150 (Laos) - - Jacoby, 1908, Fauna India, Coleopt. 2: 397, fig. 137 (Nepal, India, Burma, Andamans, Sumatra, N. China). - Chen, 1935, Sinensia 6 (3): 365 (China, Tonkin, Nepal, India, Burma, Andamans, Sumatra). -Gressitt \& Kimoto, 1961, Pac. Insects Monogr. 1A : 258, fig. 14 (S. China, Hainan). --Chûjô, 1964, Nature and Life in SE Asia, Kyoto 3: 267 (Thailand).

Distríuтion: India, Burma, Andamans, Thailand, Cambodia, Laos, Vietnam, Malaya, Borneo, China, Hainan.

material examined. THAiland: Khao Chong, nr. Trung, 1 ex., 26 . vi. 1965, Y. Miyatkae (KU) ; Kanchanaburi, 1300 ft, 3 exs., 21. v. 1962, Kanchana buri, $2500 \mathrm{ft}, 2$ exs., 21. v. 1962 (BANGKEN). LAOS: Attopeu Prov., Houei Kong, 1 ex., 31. v. 1965, Native collr (BISHOP); Umgeb. Vientiane, 1 ex., iii-vi. 1963 (MUNCHEN). VIETNAM: Dak Song, $76 \mathrm{~km} \mathrm{SW}$ of Ban Me Thuot, $870 \mathrm{~m}$, 2 exs., 19-21. v. 1960, L. W. Quate (BISHOP).

Aoria nigripes (Baly) (Fig. 25b)

Adoxus nigripes Baly, 1860, J. Entomol. 1: 28 (Hongkong; BM).

Aoria mouhoti Baly, 1878, J. Linn. Soc. Lond., 14: 247 (Cambodia, Laos ; BM). New synonymy.

Aoria nigripes: Jacoby, 1884, Notes Leyd. Mus. 6: 203 (Sumatra). -- Fairmaire, 1888, Ann. Soc. Entomol. Fr., ser. 6, 8: 372 (Tonkin). Lefevre, 1889, Ann. Soc. Entomol. Fr., ser. 6, 9: 291, 296(Tonkin: Lien-Son ;Cambodge: Pnomh-Penh) ; 1890, Nouv. Arch. Mus. Paris, ser. 3, 2: 193 (Cambodge) ; 1904, Miss. Pavie Indo-China, ser. 2, 3: 150 (Cambodge) ; 1893, Ann. Soc. Entomol. Fr. 1893: 121 (Tonkin: Ha-Lang). -Jacoby, 1908, Fauna India, Coleopt. 2: 396 (India, Burma, China, Sumatra). -Chen, 1935, Sinensia 6 (3) : 366 (China, Tonkin, Burma, India, Sumatra). Chûjô, 1956, Philip. J. Sci. 85 (1) : 117 (Formosa). Gressitt \& Kimoto, 1961, Pac. Insects Monogr. 1A: 259 (S. China, Hainan, Tonkin).

Aoria pusilla Lefevre, 1889, Ann. Soc. Entomol. Fr., ser, 6, 9: 291 (Tonkin: Pnomh-Penh; PARIS) ; 1893, ibid. 1893: 121 (Tonkin).

Aoria nigrita Jacoby, 1892, Ann. Mus. Civ. Genova 32: 904 (Burma ; GENOVA) ; 1908, Fauna India, Coleopt. 2: 397 (Burma). New synonymy.

Aoria atra Pic, 1923, Mel. Exot. Entomol. 40: 17(Tonkin; with var. scutellaris; PARIS). New synonymy.

Aoria atra varr. bicolor, bicoripes Pic, 1935, Échange 51: 8 (Tonkin).

Aoria nigrita taiwana Chûjô, 1956, Philip. J. Sci. 85 (1) : 119 (Formosa).

Distribution : India, Andamans, Burma, Thailand, Cambodia, Laos, Vietnam,

S. China, Hainan, Taiwan, Sumatra. 

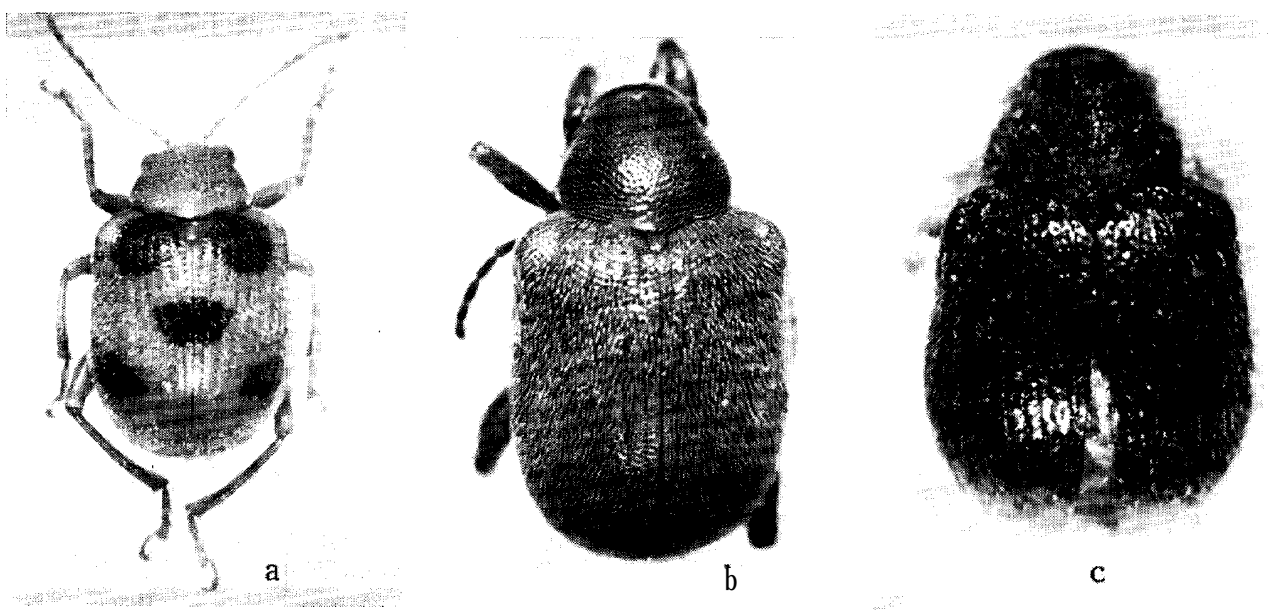

Fig. 25. a, A oria bowringii Baly ; b, A. nigripes Baly ; c, Xanthonia minuta (Pic).

Material examined. THAildnd: Doi Suthep, Chiang Mai Prov., 1 ex., 12. vi. 1965, K. Morimoto, 7 exs., 18-19. vi. 1965, K. Morimoto \& Y. Miyatake; Fang, 5 exs., 14. vi. 1965, K. Morimoto \& Y. Miyatake; Chieng Dao, 2 exs., 15. vi. 1965, K. Morimoto \& Y. Miyatake (KU) ; Chiang Mai, Doi Suthep, 1278 m, iii. - 4. v. 1958, T. C. Maa; Chiang Mai, Ban-tin-doi, 350 m, 1 ex., 13. xi. 1957, J. L. Gressitt (BISHOP). LAOS: Vientiane Prov., Ban Van Eue, 1 ex., 30. ix. 1965, Native collr (BISHOP). CAMBODIA: Damrey Phong, 1 ex., 14-16. iv. 1961, N. R. Spencer (BISHOP). VIETNAM: Fyan, 900-1000 m, 2 exs., 11. vii.9. viii. 1961, N. R. Spencer; $17 \mathrm{~km} \mathrm{~S}$ of Dilinh, $1300 \mathrm{~m}, 1$ ex., 6-13. x. 1960, C. M. Yoshimoto; $33 \mathrm{~km} \mathrm{NE}$ of Ban Me Thuot, $500 \mathrm{~m}, 1$ ex., 16-18. v. 1960, L. W. Quate (BISHOP) ; Hoa-Binh, Tonkin, 11 exs., A. Cooman (FREY).

\section{Genus Xanthonia Baly}

Xanthonia Baly, 1863, J. Entomol. 2: 151 (type: Xunthonia stevensi Baly, =villosuls Melsheimer ; U.S.A.). -Chapuis, 1874, Genera Coleopt. 10: 273. -Lefèvre, 1885, Mém. Soc. Sci. Liege, ser. 2, 11: 74. -Chen, 1935, Sinensia 6 (3) : 359; 1940, ibid. 11 (5-6) : 491.-Chûjô, 1956, Philip. J. Sci. 85 (1) : 108. -Gressitt \& Kimoto, 1961, Pac. Insects Monogr. 1A: 255. --Kimoto, 1964, J. Fac. Agr. Kyushu Univ. 13 (2) : 260.

Microlypesthes Pic, 1936, Mel. Exot. Entomol. 67: 15 (type: Microlypesthes coomani Pic, from Tonkin).- -Gressitt \& Kimoto, 1961, Pac. Insects Monogr. 1A: 267. New synonymy.

\section{Key to SPECIES of Xanthonia}

1(0). Elytron impressed by regularly arranged eleven longitudinal rows of punctures; legs entirely pale

Elytron impressed by partly arranged longitudinal rows of punctures, and hairs covering elytral surface fairly close, fine, long; dark reddish brown to pitchy black, antenna almost entirely reddish brown; legs reddish brown with posterior portion of femora blackish in various de- 
grees, in most dark colored specimen femora almost entirely pitchy

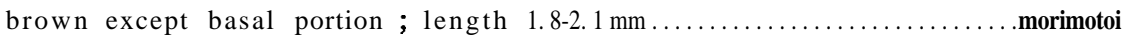

2(1). Dorsal surfaces sparsely covered with very short, fine hairs, and in well preserved specimen also covered with powdery excrescence; dark reddish brown to pitchy black, antenna and legs entirely yellowish to reddish brown; length 1.8-2.1 $\mathrm{mm}$

Dorsal surfaces thickly covered with fairly long hairs; black, with apical portion of elytron brownish, antenna and legs entirely yellowish to reddish brown, in some specimen pronotum reddish brown and elytron entirely blackish ; length 1.8-2.1 mm

minuta

\section{Xunthonia coomani (Pic) New combination (Figs. 26b-c)}

Microlypesthes coomani Pic, 1936, Mel. Exot. Entomol. 67: 16 (Tonkin; PARIS).

Distribution : Thailand, Vietnam.

Material examined. THAILAND: Chieng Dao, 1 ex., 15. vi. 1965, Y. Miyatake; Fang, 3 exs., 14. vi. 1965, K. Morimoto \& Y. Miyatake (KU) ; Fang (Agr. Exp. Station), 1 ex., 14. vi. 1965, P. D. Ashlock (BISHOP).

We could not separate the material taken from Thailand from the type of Microlypesthes coomani P ic. Chen (1940) treated several Chinese species of $M a$ legia as members of Microlypesthes. This treatment is obviously erroneous. Microlypesthes should be treated as a synonym of Xanthonia.

\section{Xanthonia minuta (Pic) (Fig. 25c)}

Aoria minuta Pic, 1929, Bull. Soc. Zool. Fr., 54: 45 (Tonkin; PARIS).

Xanthonia minuta: Chen, 1940, Sinensia 11 (5-6): 513 (S. China). Gressitt \& Kimoto, 1961, Pac. Insects Monogr. 1A: 256 (S. China, Tonkin).

Distribution : $S$. China, Vietnam, Thailand.

A specimen taken from Vietnam, differs from the nominate form in having pronotum reddish brown and elytron entirely black.

Material examined. THAiland: Doi Suthep, $1000 \mathrm{~m}$, Chieng Mai Prov., 2 exs., 18. vi. 1965, K. Morimoto \& Y. Miyatake (KU). VIETNAM: Ban Me Thuot, $500 \mathrm{~m}, 1$ ex., 20-24. xii. 1960, C. M. Yoshimoto (BISHOP).

\section{Xunthonia morimotoi Kimoto \& Gressitt, n. sp. (Fig. 26a)}

Oblong oval; dark reddish brown to pitchy black, antenna almost entirely reddish brown; legs reddish brown with posterior portion of femora blackish in various degrees, in most dark colored specimen femora almost entirely pitchy brown except basal portion.

Head finely punctate and covered by fine pubescence; clypeus distinctly raised, impressed by much larger size of punctures than vertex, slightly widened anteriorly. Antenna slender, filiform, nearly $2 / 3$ as long as body length; first segment robust, club-shaped; second shortest, nearly $2 / 3$ as long as first, and much slenderer; third nearly $13 / 5$ times as long as second, 


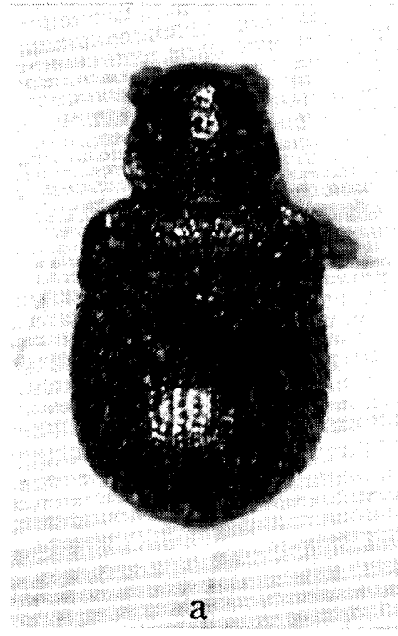

Fig. 26. a, Xanthonia morimotoi

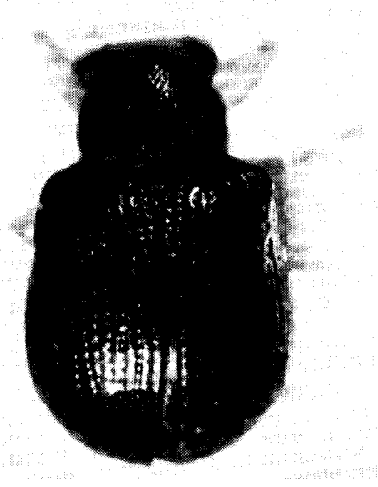

$\mathrm{b}$

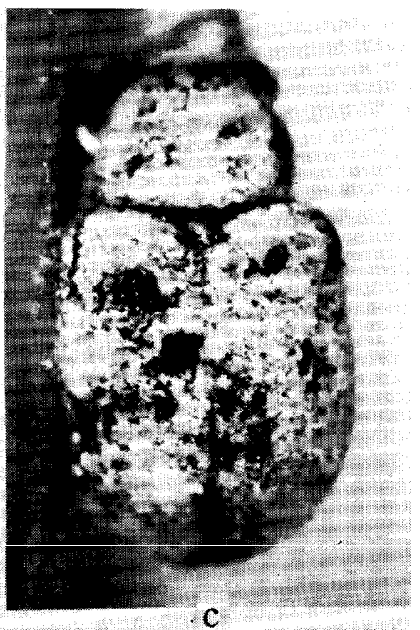

c

Kimoto \& Gressitt, n. sp.; b, X. coomani (Pic); c, ditto, holotype.

slender; fourth nearly $11 / 3$ times as long as third; fifth and sixth subequal to fourth in length and shape; seventh nearly $4 / 5$ as long as sixth and gradually widened apically; eighth slightly shorter than seventh but slightly robuster; ninth subequal to eighth in length and shape; tenth slightly shorter than ninth; eleventh slightly longer than tenth and its apex pointed. Pronoturn transverse, nearly $11 / 3$ times as wide as long, rounded at side, widest almost at middle, lateral border not marginate; dorsum strongly convex, with a broad, transverse depression anteriorly, which is interrupted at middle, closely and strongly punctate throughout. Scutellum trapezoidal, narrowed posteriorly, surface closely punctate and pubescent. Elytron much wider than pronotum at base, slightly widened posteriorly and rounded at apex; dorsum convex, impressed with partly arranged longitudinal rows of punctures, and hairs covering surface fairly close, fine and long.

Length $1.8-2.1 \mathrm{~mm}$.

Holotype (Type No. 2357, Kyushu Univ.), THAILAND: Chiang Mai Prov., Chiang Dao, 16. vi. 1965, K. Morimoto. Paratopotypes: 3 exs., same data as the holotype (KU, BISHOP, KIMOTO). Paratypes: THAILAND: 6 exs., same data as the holotype but 15. vi. 1965, K. Morimoto \& Y. Miyatake; 1 ex., Doi Suthep, $1000 \mathrm{~m}, 1$ ex., 18. vi. 1965, Y. Miyatake (KU),

This new species somewhat resembles Xanthonia sinica Chen, but differs in having the head more closely covered with hairs and the legs brownish with femora blackish in various degrees.

\section{Genus Goniopleura Westwood}

Goniopleura Westw., 1832, in Griffith, Animal Kingdom Arranged in Conformity, 15: 149 
(type: Goniopleura auricoma Westwood, from Penang). -Chapuis, 1875, Genera Coleopt. 11: 248 (Galerucinae). -Gahan, 1895, Ann. Mag. Nat. Hist. ser. 6, 15: 454.

\section{KeY TO SPECIES OF Goniopleura}

$1(0)$. Dorsal surfaces yellowish to reddish brown with blackish markings 2 Dorsal surfaces entirely yellowish to reddish brown; legs yellowish brown with tibiae, tarsi and apex of femora blackish; antenna black-

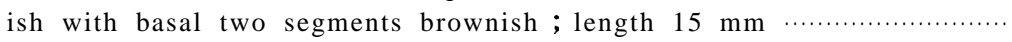

2 (1). Antenna normal, elytron with a lateral costa subbasally, starting from humerus and ending almost at middle ; reddish brown, elytron yellowish brown with lateral and sutural markings blackish; antenna pitchy black with basal segments brownish; legs pitchy black with middle of

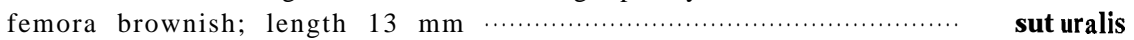

Antenna with basal segments robust, depressed; elytron without lateral costa; reddish brown, elytron with scutellar and humeral markings blackish, antenna pitchy black with basal segments brownish, in dark colored specimen pronotum pitchy brown, antenna almost entirely pitchy black; legs pitchy black with middle of femora brownish; length $15 \mathrm{~mm}$

tonkinea

\section{Goniopleura moseri Weise}

Goniopleura moseri Weise, 1922, Tijdschr. Entomol. 65: 50 (Tonkin; STOCKHOLM).

Distribution : Vietnam.

No additional material.

\section{Goniopleura suturalis Pic}

Goniopleura suturalis Pic, 1928, Mé1. Exot. Entomol. 52: 26 (Tonkin; PARIS).

Distribution : Vietnam.

\section{Goniopleura tonkinea Pic (Fig. 27a)}

Goniopleuratonkinea Pic, 1928, Mél. Exot. Entomol. 52 : '26 (Tonkin ; PARIS).

Distribution : Laos, Vietnam.

Material EXAMINED. LAOS: Ban Van Heue, 20km E of Phou-kow-kuei, 1 ex., 15-31. iv. 1965, Native collr (BISHOP). “Indo-China”, 1 ex. (BASEL).

It is possible that G. suturalis is' nothing but a female specimen of tonkinea Pic.

\section{Genus Aulexis Baly}

Aulexis Baly, 1863, J. Entomol. 2: 153 (type : Aulexis nigricollis Baly, from Borneo), -Chapuis, 1874, Genera Coleopt. 10: 276. -Lefèvre, 1885, Mém. Soc. Sci. Liège, ser. 2, 11: 76. Jacoby, 1908, Fauna India, Coleopt. 2: 410. - Chen, 1935, Sinensia 6 (3) : 355 ; 1940, ibid. 11 (5-6) : 491. -Gressitt \& Kimoto, 1961, Pac. Insects Monogr. 1A: 26`2. 


\section{KeY to SPECIES OF Aulexis}

$1(0)$. Elytron without any distinct costa laterally

Elytron with a distinct lateral costa, starting from humerus and ending at apex; brownish with elytron blackish brown, tibiae infuscate; length $6.0 \mathrm{~mm}$

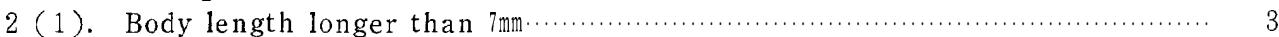

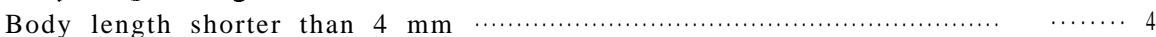

3 (2). Entirely yellowish brown, antenna with apical segments infuscate ; prothorax with three teeth laterally; length $7.0 \mathrm{~mm} \ldots \ldots \ldots \ldots \ldots \ldots \ldots \ldots \ldots$. languei

Reddish brown, head and prothorax blackish; elytron yellowish brown; prothorax with three teeth laterally; length $7.0 \mathrm{~mm} \ldots \ldots \ldots \ldots \ldots \ldots \ldots . . . . . . .6$ bicolor

4 (2). Antenna robust, nearly twice or $11 / 2$ times as long as wide at preapical

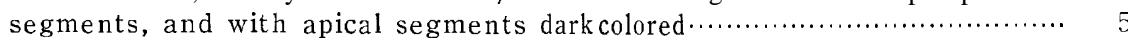

Antenna slender, nearly three times as long as wide; general color yellowish brown, antenna entirely pale ; length 3.5-4. $5 \mathrm{~mm}$

unispinosa

5 (4). Each side of prothorax with three distinct, well separate teeth, and prothorax more transverse, nearly $13 / 5$ times as wide as long; general color reddish brown, elytron mostly pitchy black, antenna pitchy black with basal segments brownish; legs pitchy black with femora mostly brownish ; length $4.0 \mathrm{~mm}$

nigripennis

Each side of prothorax with two minute, more or less fused teeth at middle, and prothorax rather short, nearly $11 / 4$ times as wide as long; general color reddish brown, antenna with apical segments pitchy brown ; length $2.8 \mathrm{~mm}$

minor

\section{Aulexis bicolor Pic}

A ulexis bicolor Pic, 1927, Mél. Exot. Entomol. 54: 15 (Tonkin ; PARIS).

Distribution : Vietnam.

No additional material.

Aulexis carinata Pic

Aulexis carinata Pic, 1935, Échange 51: 8 (Tonkin; PARIS).

Distributeon : Vietnam.

No additional material.

\section{Aulexis humeralis Le fèvr e}

Aulexis humeralis Lef., 1885, Mém.Soc. Sci. Liege, ser. 2, 11: 77, nota 1 (Siam: Ile Juthia).

Distribution : Thailand.

We could not trace the type location of this species. This species is not included in the key. It is possible that this species is proved as a same species with unispinosa Pic.

\section{Aulexis languei Lefèvre}

Aulexis languei Lef., 1893, Ann. Soc. Entomol. Fr. 62: 121 (Tonkin; PARIS). 
Distribution : Vietnam.

No additional material.

Aulexis minor Kimoto \& Gressitt, n. sp. (Fig. 27c)

Oblong oval; general color yellowish to reddish brown, antenna pitchy brown with five or six basal segments reddish brown.

Head with surface distinctly and rather closely punctate on clypeus and more finer and sparser on vertex, and interstices of punctures smooth, shining. Antenna nearly $2 / 3$ as long as body length, rather robuster, distinctly thickened in apical segments ; first segment robust, somewhat club-shaped; second nearly $2 / 3$ as long as first, and nearly twice as long as wide; third slightly longer than second but much slenderer; fourth subequal to third in length and shape; fifth slightly longer and robuster than fourth; sixth subequal to fifth in length and shape; seventh subequal to sixth in length but gradually widened apically; eighth slightly shorter than seventh and nearly twice as long as wide; ninth and tenth subequal to eighth in length and shape; eleventh $11 / 2$ times as long as tenth and its apex pointed. Pronotum transverse, nearly $11 / 4$ times as wide as long, and armed at middle of each side with two minute, more or less closely situated teeth; disc fairly smooth and even, distinctly and not very closely punctate, and with a pair of shallow oblique depressions laterally. Scutellum convex, subpentagonal, narrowed behind and rounded apically, surfaces distinctly punctate. Elytron suboblong, broadly rounded; surface distinctly and somewhat closely punctate.

Length $2.8 \mathrm{~mm}$.

Holotype (Type No. 2358, Kyushu Univ.), THAILAND: Kao Chong, nr. Trung, 26. vi. 1965, Y. Miyatake. Paratopotype: 1 ex., same data as the holotype (BISHOP).

This new species somewhat resembles A. brevidentata Gressitt from China, but is separable in being the body length shorter and the body entirely reddish brown.

\section{Aulexis nigripennis Kimoto \& Gressitt, n. sp. (Fig. 27b)}

Oblong oval; general color reddish brown, elytron pitchy black with basal margin more brownish; antenna pitchy black with five or six basal basal segments brownish; legs pitchy black with femora mostly reddish brown.

Head with surface distinctly and rather closely punctate and interstices of punctures smooth, and shining. Antenna nearly $2 / 3$ as long as body length, rather robuster, distinctly thickened in apical segments; first segment robust, somewhat club-shaped; second nearly $3 / 4$ as long as first, and nearly twice as long as wide; third slightly longer than second but much slenderer; fourth subequal to third in length and shape; fifth nearly $3 / 4$ as long as fourth; 

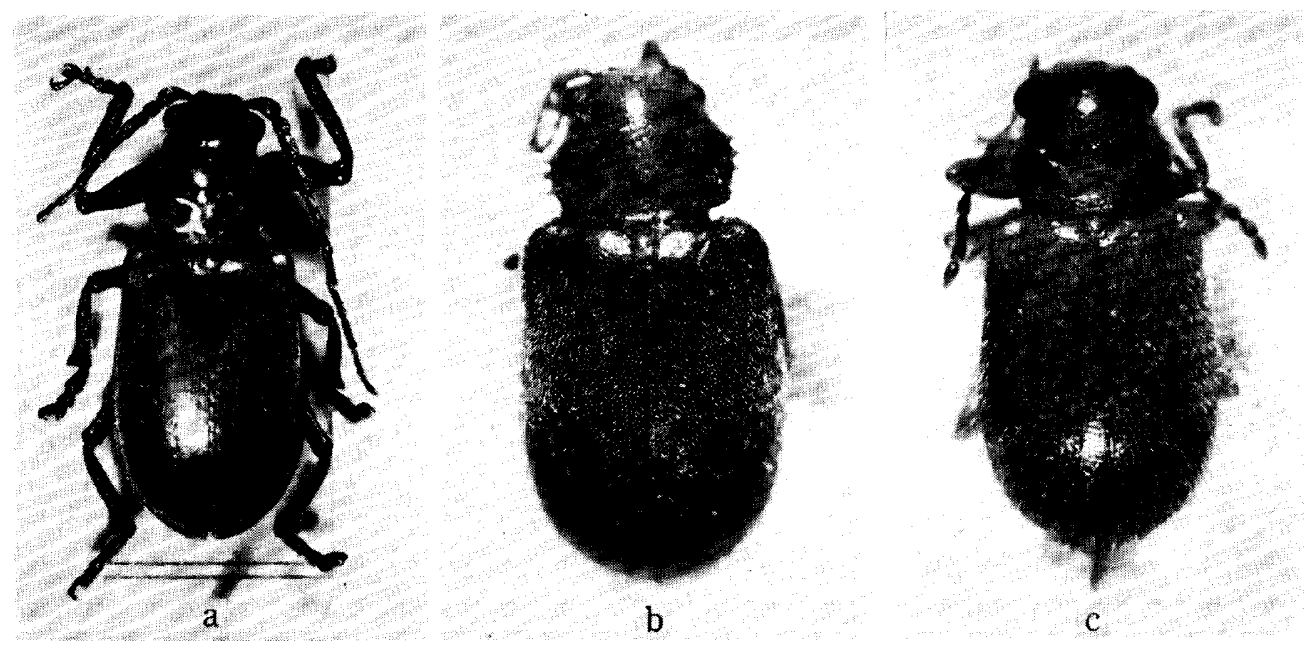

Fig. 27. a, Goniopleura tonkinen Pic; b, Aulexis nigripennis Kimoto \& Gressitt, n. sp. ; c, A. minor Kimoto \& Gressitt, n. sp.

sixth subequal to fifth in length and shape; seventh nearly $12 / 3$ times as long as sixth, widened apically; eighth nearly $4 / 5$ as long as seventh, and $11 / 3$ times as long as wide; ninth and tenth subequal to eighth in length and shape; eleventh nearly $1 \frac{1 / 2}{2}$ times as long as tenth and its apex pointed. Pronotum transverse, nearly $13 / 5$ times as wide as long, and armed at middle of each side with three distinct, well separate teeth, which are nearly equal in length; disc fairly smooth and even, distinctly and rather closely punctate, and with a pair of shallow oblique depressions laterally. Scutellum convex, subpentagonal, narrowed behind and rounded apically, surface distinctly punctate. Elytron suboblong, broadly rounded ; surface distinctly and somewhat closely punctate.

Length $4.0 \mathrm{~mm}$.

Holotype (BISHOP), VIETNAM: $60 \mathrm{~km}$ SW, Blao (Balao), $500 \mathrm{~m}, 22$. iv. 1960, L. W. Quate. Paratopotype : 1 ex., same data as the holotype (KIMOTO).

This new species somewhat resembles Aulexis atripennis Pic, from China, but differs in having the ventral surfaces of body entirely brownish.

\section{Aulexis unispinosa Pic}

Aulexis unispinosaPic, 1935, Bull. Soc. Zool. Fr. 60: 172 (Tonkin; PARIS).

Distribution : Vietnam.

The number and shape of teeth on the side prothorax seem to be unstable character.

Material examined. THAiland: Doi Suthep, $1000 \mathrm{~m}$, Chiang Mai Prov., 4 exs., 18. vi. 1965, K. Morimoto \& Y. Miyatake (KU). CAMBODIA: Domrey 
Phong, 1 ex., 14-16. iv. 1961, N. R. Spencer (BISHOP). LAOS: Ban Van Heue, $20 \mathrm{~km}$ E of Phou-kow-kuei, 3 exs., 1-15. v. 1965, J. A. Rondon (BISHOP). VIETNAM : Fyan, 900-1000 m, 14. exs., 11. vii. - 9. viii. 1961, N. R. Spencer ; Fyan, $1200 \mathrm{~m}, 5$ exs., 11. vii. - 9. viii. 1961, N. R. Spencer; $7 \mathrm{~km}$ SE of Dilinh (Djiring), 990 m, 2 exs., 2. v. 1960, R. E. Leech; Dilinh, 1 ex., 27. ix. 14. x. 1960, C. M. Yoshimoto; Blao (Balao), 500 m, 2 ess., 14-21. x. 1960, C. M. Yoshimot o (BISHOP).

\section{Genus Abirus C hapuis}

Abirus Chapuis, 1874, Genera Coleopt. 10: 310 (type :Cryptocephalus aeneus Wiedermann, from Java). --Lefèvre, 1885, Mém. Soc. Sci. Liege, ser. 2, 11: 13. -Jacoby, 1908, Fauna India, Coleopt. 2: 456. -Chen, 1935, Sinensia 6(3): 259; 1940, ibid. 11(5-6) : 492. -Chûjô, 1956, Philip. J. Sci. 85 (1): 128. -Gressitt \& Kimoto, 1961, Pac. Insects Monogr. $1 \mathrm{~A}:$ 277. -Kimoto, 1964, J. Fac. Agr. Kyushu Univ. 13 (2): 236.

\section{Abirus fortuneii (Baly) (Fig. 2c)}

Dermorytis fortuneii Baly, 1861, J. Entomol. 1: 283 (N. China ; BM).

A birus harmandi Lefevre, 1876, Rev. Mag. et Zool. ser. 3, 4: 305 (Saigon). -Jacoby, 1889, Ann. Mus. Civ. Genova, ser. 2, 7: 167 (Burma). -Lefèvre, 1889, Ann. Soc. Entomol. Fr. ser. 6, 9: 296 (Tonkin:Lienson) ; 1890, Nouv. Arch. Mus. Paris, ser. 3, 2: 196 (Laos) ; 1904, Miss. Pavie Indo-China, ser. 2, 3 (Zool.) : 152 (Laos). New synonymy.

A birus granosus Lefevre, 1893, Ann. Soc. Entomol. Fr. 62: 128 (Tonkin). New synonymy. Abirus denticollis Lefevre, 1893, Ann. Soc. Entomol. Fr. 62: 127 (Tonkin). New synonymy.

Abirus harmandi var. archardi Pic, 1923, Mél. Exot. Entomol. 39: 12 (Tonkin).

Abirus harmandi varr. viridenscens, cuprescens, curtus Pic, 1946, Échange 62: 13 (Tonkin). Abirus recticollis Pic, 1927, Bull. Soc. Linn. Lyon 6: 133 (China: Fookien ; PARIS). -Gressitt \& Kimoto, 1961, Pac. Insects Monogr. 1A: 278 (=fortuneii).

Abirus sinensis Pic, 1927, Buil. Soc. Linn. Lyon 6: 133 (Yunnan ; PARIS). -Gressitt \& Kimoto, 1961, Pac. Insects Monogr. 1A: 278 (=) fortuneii).

Abirus atricolor Pic, 1927, Mel. Exot. Entomol. 49: 23 (Tonkin; with var. superbus; PARIS). New synonymy.

Abirus yashiroi Yuasa, 1930, Proc. Imp. Acad. Tokyo 6 (7) : 294, fig. 2 (Okinawa). Chûjô \& Kimoto, 1961, Pac. Insects 3 (1) : 139 (=fortuneii).

Abirus kiotoensis Pic, 1944, Échange 60: 8 ("Kioto" ; PARIS). - Chûjô \& Kimoto, 1961, Pac. Insects 3 (1) : 139 (=fortuneii).

Abirus mallet $i$ Pic, 1944, Échange 60: 8 (Tonkin; PARIS). N ew synonymy.

A birus sinensis var. guerryi Pic, 1946, Échange 62: 14 (China).

Abirus fortuneii: Fairmaire, 1889, Ann. Soc. Entomol. Fr. ser. 6, 9: 71 (China, Korea). --Weise, 1922, Tijdschr. Entomol. 65: 51 (China). Chen, 1935, Sinensia 6 (3): 260 (Korea, China). - Chûjô, 1956, Philip. J. Sci. 85 (1) : 131 (Formosa). -Gressitt \& Kimoto, 1963, Pac. Insects Monogr. 1A: 277 (China, Tonkin). --Kimoto, 1964, J. Fac. Agr. Kyushu Univ. 13 (2): 237 (Ryukyu Is.).

Distribution : Burma, Thailand, Laos, Vietnam, Ryukyus, Taiwan, China,

Korea.

Elongate ; subcylindrical, dorsal surfaces covered with fine pubescence; pronotum and elytron closely impressed with large punctures, and the latter with strongly raised transverse rugosities. General color variable : metallic 
green, cupreous, violaceous or purplish; antenna blackish brown with basal segments fulvous ; length 7.5-9.5 mm.

Material examined. THAiland: Chiang Mai Prov., 1 ex., 15. vi. 1965, P. D. Ashlock (BISHOP). LAOS: Sedone Prov., Pakse, 1 ex., 16. v. 1965, Native collr ; Sedone Prov., $5 \mathrm{~km} \mathrm{E}$ of Pakse, 1 ex., 13. v. 1965, P. D. Ashlock; Wapikhamthong Prov., Khong Sedone, 1 ex., 16. v. 1965, Native collr; Sayaboury Prov., Sayaboury, 1 ex., 20. v. 1966; Khammouane Prov., Phon Tiou, 1 ex., 28. iv. 1965, 1 ex., 17. v. 1965, Native collr (BISHOP) ; Umgeb. Vientiane, 31 exs., 1963; Umgeb. Paklay, 6 exs., 1963; Umgeb. Vanky, 3 exs., 1964; Umgeb. Pakse, 1 ex., 1963 (MUNCHEN). VIETNAM: Ban Me Thuot, 6 exs., 16-18. v. 1960, L. W. Quate, S. Quate, \& R. E. Leech (BISHOP) ; Hoa-Binh, Tonkin, 13 exs., A. Cooman; Chapa, Tonkin, 7 exs., A. Cooman, 2 exs., 23-24. iv. 1918, Jeanvoine; Hanoi, Tonkin, 5 exs., 13-22. iii. 1918, Jeanvoine (FREY).

\section{Genus Pachnephorus Chevrolat}

Pachnephorus Chevr., 1837, in Dejean, Cat. Coleopt. ed. 3: 408. -Chapuis, 1874, Genera Coleopt. 10: 323. --Lefèvre, 1885, Mém. Soc. Sci. Liege, ser. 2, 11: 123. -Jacoby, 1908, Fauna India, Coleopt. 2: 460 (type :Cryptocephalus pilosus Rossi, from Europe). --Chen, 1935, Sinensia 6 (3) : 277; 1940, ibid. 11 (5-6) : 489. -Monórs \& Bechyné, 1956, Entomol. Arb. Mus. Frey 7 (3) : 1127 (type : Cryptocephalus arenarius Fabricius =pilosus). - Chû jô, 1959, Philip. J. Sci. 85 (1) : 142. --Gressitt \& Kimoto, 1961, Pac. Insects Monogr. 1A : 239.

\section{Key to SPECIEs of Pachnephorus}

Robust ; pronotum short ; elytral punctures partly irregular ; length 2. 4-3.5 mm ….. lewisii

Slender ; pronotum elongate ; elytral punctures regular, punctural interstices with minute punctures; length $2.3-3.0 \mathrm{~mm}$

porosus

Pachnephorus lewisii Baly (Fig. 28b)

Pachnephorus Zewisii Baly, 1878, J. Linn. Soc. Lond., 2001. 14: 257 (China ; BM). -Gressitt \& Kimoto, 1961, Pac. Insects Monogr. 1A: 240 (China).

Pachnephorus bretinghami Baly, 1878, J. Linn. Soc. Lond., Zool. 14 : 256 (India ; BM). -Jacoby, 1908, Fauna India, Coleopt. 2: 460 (India, Burma, Sumatra). --Weise, 1922, Philip. J. Sci. 21 (5) : 481 (Philippines). - Chen, 1935, Sinensia 6 (3): 279 (Fokien, Burma, India, Sumatra). New synonymy.

Pachnephorus variegatus Lefèvre, 1887, Bull. Soc. Entomol. Fr. ser. 6, 7: 57 (Cochinchina : env. de Hue) ; 1889, Ann. Soc. Entomol. Fr. ser. 6, 9: 293 (Annam: Hue ; Cochinchina : Saigon ; Cambodge : Pnomh-Penh) ; 1893, ibid. 1893: 128 (Tonkin). New synonymy.

Pachnephorus plagiatus Jacoby, 1892, Ann. Mus. Civ. Genova 32: 913 (Burma ; BM, GENOVA) ; 1899, Stett. Entomol. Zeit. 60: 313 (=bretinghami).

Pachnephorus formosanus Chû jô, 1938, Arb. Morph. Taxon. Entomol. Berlin-Dahlem 1 (5) : 32 (Formosa ;TARI) ; 1959, Philip. J. Sci. 85 (1) : 145 (Formosa). - Kimoto, 1969, Esakia, Kyushu Univ. 7: 18 (=lewisii).

Distribution: India, Burma, Thailand, Cambodia, Laos, Vietnam, China, Taiwan. 
Material examined. THAiland: Chiang Mai Prov., Maesa Water Fall, 1 ex., 16. vi. 1965, K. Morimoto; Chiang Mai, 1 ex.. 9. vi. 1965, K. Morimoto ; Chiang Dao, 1 ex., 15. vi. 1965, K. Morimoto; Khao Yai Nat. Park, 1 ex., 6. vi. 1965, K. Moriotmo (KU). CAMBODIA: Ph. Chisau, $40 \mathrm{~km} \mathrm{~S}$ of P. Penh, $20 \mathrm{~m}$, 1 ex., 29. iv. 1961, N. R. Spencer (BISHOP). LAOS: Vientiane Prov., Vientiane, 1 ex., 28. vii. 1965, Native collr; Sedone Prov., Pakse, 2 exs., 23. v. 1965, P. D. Ashlock; Nongtevada, 1 ex., 27. vi. 1965, Native collr, 1 ex., 6 viii. 1965, Native collr (BISHOP). V I E T NAM : Hoa-Binh, Tonkin, 1 ex., A. Cooman (FREY).

Our 1961 identification of bretinghami Baly should be corrected as porosus Baly and part of Zewisii as porosus Baly.

\section{Pachnephorus porosus Baly Resurrected from synonymy (Fig. 28a)}

Pachnephorus porosus Baly, 1878, J. Linn. Soc. Lond., 2001. 14: 256 (China ; BM). --Gressitt\& Kimoto, 1961, Pac Insects Monogr. 1A: 240 (erroneously synonymized as bretinghami). Pachnephorus seriatus Lefèvre, 1887, Bull. Soc. Entomol. Fr., ser. 6, 7: 57 (Cochinchina: env. de Hué; cotype : BM) ; 1889, Ann. Soc. Entomol. ser. 6, 9: 293 (Annam;Hué). -Fairmaire, 1889, Ann. Soc. Entomol. Belg. 32: 39 (Fokien). --Lefèvre, 1889, Ann. Soc. Entomol. Fr., ser. 6. 9: 293 (Annam). -Weise, 1922, Tijdschr. Entomol. 65: 52 (Fokien). -Chen, 1935, Sinensia 6 (3) : 278 (Fokien, Tonkin, Cochinchina). -Gressitt \& Kimoto, 1961, Pac. Insects Monogr. 1A: 240 (China). New synonymy.

Pachnephorus squamosus Chûjô, 1936, Bull. Umeno Entomol. Lab. 3: 9 (Korea ;TARI). -Takizawa, 1980, Nature \& Life, Kyunpook J. Biol. Sci. 10 (2) : 5 (Korea). New synonymy.

Pachnephorus sauteri Chûjô, 1938, Arb. Morph. Taxon. Entomol. Berlin-Dahlem 1 (5) : 32 (Formosa ;TARI) ; 1956, Philip. J. Sci. 85 (1) : 146 (Formosa). -Kimoto, 1969, Esakia, Kyushu Univ. 7: 19 (erroneously synonymized as bretinghami). New synonymy.

Pachnephorus bretinghami: Gressitt \& Kimoto, 1961, Pac. Insects Monogr. 1A: 240, fig. 60a (China). --Kimoto, 1969, Esakia, Kyushu Univ. 7: 19 (Taiwan). -Gruev, 1978, Entomol. Rev. Japan 32 (1-2) : 51 (Korea).

Pachnephorus Zewisii: Gressitt \& Kimoto, 1969, Pac. Insects Monogr. 1A: 240 (in part ; Kirin, E. Siberia).

Distribution : India, Burma, Thailand, Laos, Vietnam, China, Taiwan, Korea, E. Siberia.

Material Examined. THAILAND: Chiangmai Prov., Chiangmai (Arboretum), 300 m, 1 ex., 12. vi. 1965, P. D. Ashlock (BISHOP). LAOS: Sedone Prov., Pakse, 1 ex., 14. v. 1965, P. D. Ashlock; 1 ex., 18. v. 1965, P. D. Ashlock (BISHOP).

\section{Genus Aulacolepis Baly}

Aulacolepis Baly, 1863, J. Entomol. 2: 162 (type : Aulacolepis mouhoti Baly, from Siam). -Chapuis, 1874, Genera Coleopt. 10: 327. -Lefèvre, 1885, M\&m. Soc. Sci. Liège, ser. 2, 11: 127. -Jacoby, 1908, Fauna India, Coleopt. 2: 431.

Aulacolepis mouhoti Baly (Figs. 29a-b)

Aulacolepis mouhoti Baly, 1863, J. Entomol. 2 :168 (Siam ; BM). Lefèvre, 1889, Ann. Soc. 

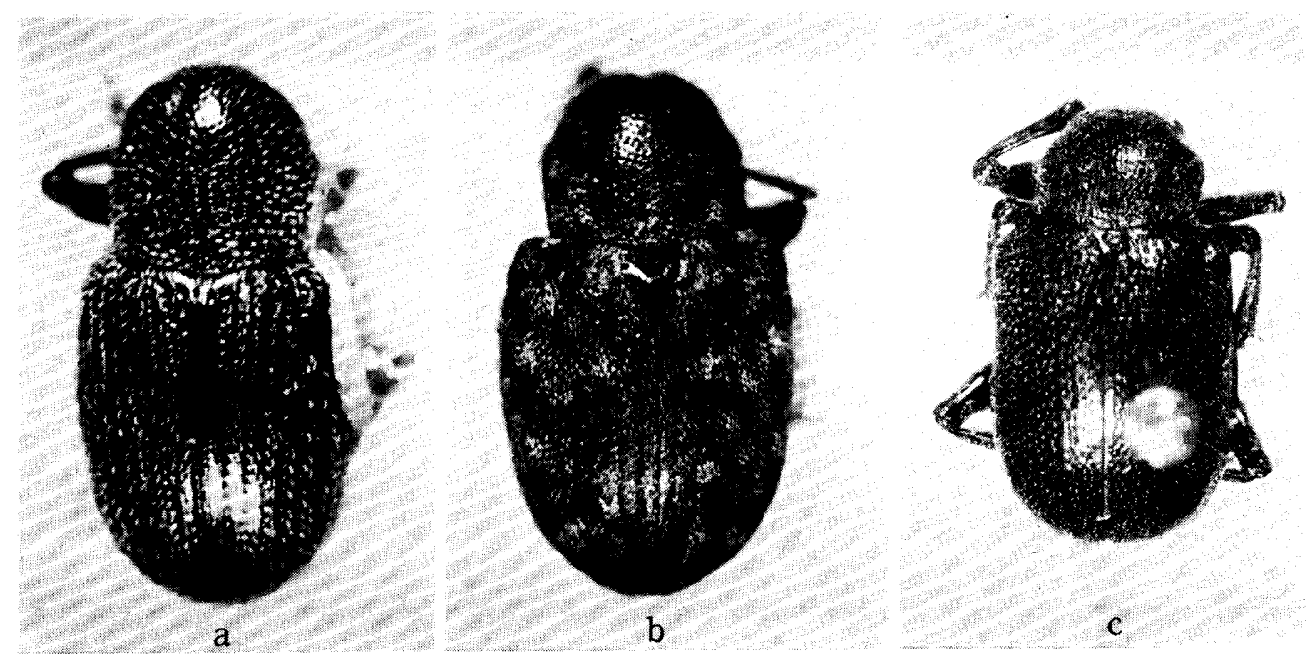

Fig. 28. a, Pachnephorus porosus Baly; b, P. lewisiiBaly; c, Lophea melancholica Baly.

Entomol. Fr., ser. 6, 9: 293 (Cochinchina; Saigon) ; 1893, Ann. Soc. Entomol. Fr., 1893: 128 (Bords du Meking et montagnes de Chaudoc). --Jacoby, 1908, Fauna India, Coleopt. 2: 432, fig. 152 (Burma, Siam).

Distribution : Burma, Thailand, “Indo-China”.

Body oblong, subnitid, black ; closely covered with large, broad, rigid, adpressed or suberect scales, which are white, brownish or blackish, surface of scales longitudinally concave; pronotum with a pair of elevated large tubercles at middle; elytron with about seven tufts of rigid, erect, black scales; length 5.0-5.5 $\mathrm{mm}$.

Material examined. THAiland: Fang, 1 ex., 14. vi. 1965, K. Morimoto ; Khao Chong, nr. Trung, 1 ex., 26. vi. 1965, Y. Miyatake (KU).

\section{Genus Lophea Baly}

Lophea Baly, 1865, J. Entomol. 2: 441 (type : Lophea melancholica Baly, from Burma). -Chapuis, 1874, Genera Coleopt. 10: 316.-Lefèvre, 1885, Mém. Soc. Sci. Liege, ser. 2, 11: 17. Jacoby, 1908, Fauna India, Coleopt. 2: 462.

\section{Key to SPECIES OF Lophea}

Elytral surface strongly and closely punctate and their interstices flat; greenish, violaceous, bluish black; length $8 . \mathrm{B}-10.7 \mathrm{~mm} \ldots \ldots \ldots \ldots \ldots \ldots \ldots \ldots \ldots \ldots \ldots \ldots \ldots \ldots$ melancholica

Elytral surface strongly punctate, and their interstices convex and irregularly ru-

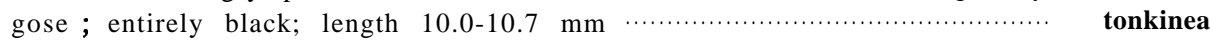

\section{Lophea melancholica Baly (Fig. 28c)}

Lophea melancholica Baly, 1865, J. Entomol. 2: 442 (Burma ; BM).

Chrysochus hirsutus Jacoby, 1900, Mém. Soc. Entomol. Belg. 7: 117 (Burma). -Bryant, 1337, 

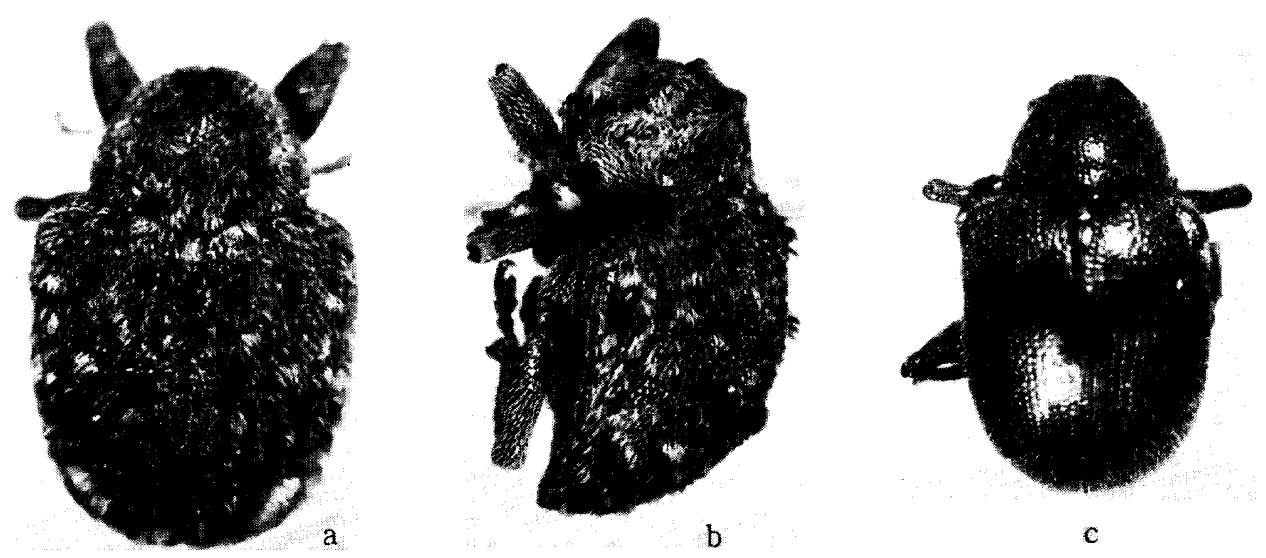

Fig. 29. a, Aulacolepis mouhoti Baly, dorsal view; b, ibid., lateral view; c, H eterotrichus balyi Chapuis.

Ann. Mag. Nat. Hist. ser. 10, 20: 97 (=melancholica).

Dermorhytis hirsuta: Jacoby, 1908, Fauna India, Coleopt. 2: 377 (Burma).

Lophea salvazai Pic, 1928, Mél. Exot. Entomol. 52: 28 (Laos ;?PARIS). New synonymy.

Distribution : Burma, Thailand, Laos.

We could not find the type of salvazai Pic but a series of material identified by Pic in Paris Museum. The material should be identified as melancholica Baly.

MAterial ExAmined. THAILAND: Fang, 1 ex., 14. vi. 1965, Y. Miyatake (KU). LAOS: Vientiane Prov., Ban Van Eue, 1 ex., 29. iv. 1966, 1 ex., 15. iv. 1966, Native collr ; Sayaboury Prov., Sayaboury, 1 ex., 5. v. 1966, 1 ex., 20. v. 1966, Native collr; Kyouang Prov., Ban Sam Thang, 1 ex., 5. vi. 1965, Native collr (BISHOP).

\section{Lophea tonkinea Pic}

Lophea tonkinea Pic, 1927, Bull, Soc. Linn. Lyon 6: 133 (Tonkin; PARIS).

Distribution: Vietnam,

Material examined. VietNAM: Hoa Binh, Tonkin, 13 exs., A. Cooman (FREY).

\section{Genus Heterotrichus Chapuis}

Heterotrichus Chap., 1874, Genera Cloeopt. 10: 316 (type: Heterotrichus balyi Chapuis, from Siam). -Lefèvre, 1885, Mém. Soc. Sci. Liege, ser. 2, 11: 118. - Jacoby, 1908, Fauna India, Coleopt. 2: 463. 
Heterotrichus balyi Chapuis (Figs. 29c, 30a)

Heterotrichus balyi Chap., 1874, Genera Coleopt. 10: 317, nota 1, pl. 121, fig. 6 (Juthia; BRUXE-

LES). -Lefèvre, 1890, Nouv. Arch. Mus. Paris, ser. 3, 2: 197 (Laos) ; 1904, Miss. Pavie

Indo-China, ser. 2, 3 (2001.) : 152 (Laos). -Jacoby, 1908, Fauna India, Coleopt. 2: 464,

pl. 2, fig. 2 (Burma, Siam).

Trichochrysea laosensis Pic, 1928, Mél. Exot. Entomol. 52 : 27 (Laos ; PARIS) . New synonymy.

Distribution : Burma, Thailand, Laos, Vietnam.

Short ovate, thickly covered with erect hairs; head closely and rugosely punctate; bluish balck, elytron golden cupreous, with suture and a broad angulate band placed near base and side bluish black, but in some specimen elytron entirely bluish black ; length $5.3-7.1 \mathrm{~mm}$.

Material examined. THAiland: Chiang Mai Prov., Fang (Agr. Expt. Station), 600 m, 1 ex., 14. vi. 1965, P. D. Ashlock (BISHOP) ; Mae Sa Water Fall, Chiang Mai Prov., 1 ex., 16. vi. 1965, Y. Miyatkae ; Tan Keo, Doi Suthep, 800 m, Chiang Mai Prov., 1 ex., 10. vi. 1965, Y. Miyatake (KU) ; Sam Ngow, Tak, 1 ex., 23. vi. 1959; Kanchanaburi, 3 exs., 21. v. 1962; Utaithani, 2 exs., 13. iv. 1963 (BANGKHEN). LAOS: Sayaboury Prov., Sayaboury, 1 ex., 8. v. 1965, 1 ex., 30. v. 1965, 1 ex., 20. v. 1966, Native collr ; Wapikhamthong Prov., Khong Sedone, 1 ex., 17. vii. 1965, Native collr ; Sedone Prov., Pakse, 1 ex., 5. vii. 1965, Native collr ; $5 \mathrm{~km} \mathrm{E}$ of Pakse, 1 ex., 13. v. 1965, P. D. Ashlock; Ban Van Heue, $20 \mathrm{~km}$ E of Phou-kow-kuei, 1 ex., 1-15. v. 1965, J. A. Rondon (BISHOP). VIETNAM: Ban Me Thuot, 500 m, 3 exs., 16-18. v. 1960, S. Quate \& L. W. Quate (BISHOP).

\section{Genus Cleoporus Lefèvre}

Cleoporus Lef., 1884, Bull. Soc. Entomol. ser. 6, 4: 76 (type: Cleoporus cruciatus Lef.; Philippines) ; 1885, Mém.Soc. Sci. Liège, ser. 2, 11 : 137. -Jacoby, 1908, Fauna India, Coleopt. 2: 479. -Chen, 1935, Sinensia 6 (3) : 283; 1940, ibid. 11 (5-6) : 489. -Chûjô, 1956, Philip. J. Sci. 85 (1) : 152. - -Gressitt \& Kimoto, 1961, Pacif. Insects Monogr. 1A: 199.

Kimoto, 1964, J. Fac. Agr. Kyushu Univ. 13 (2) : 239.

\section{KeY TO SPECIES OF Cleoporus}

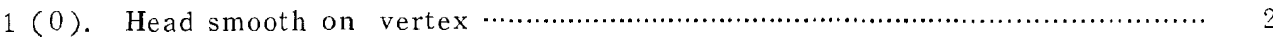

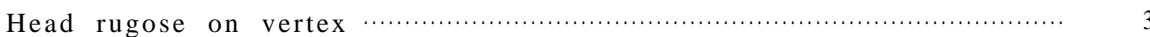

2 (1). Prothorax with side almost subparallel or widest almost at middle and slightly narrowed anteriorly and posteriorly, surface nearly impunctate; head with deep supraocular sulcus shorter and without deep and wide excavation above eye; ochraceous, in some specimen elytron black with apical portion brownish; length 2. 4-2. $9 \mathrm{~mm}$................ inornatus

Prothorax with side widest before base and distinctly narrowed anteriorly, surface somewhat finely punctured; head with deep supra-ocular sulcus longer and wide excavation above eye; very variable in coloration: (a) dorsal surfaces entirely black ; (b) black with pale pronotum, humerus and apex of elytron; (c) reddish brown with black markings on elytron; (d) reddish brown with scutellum and elytral suture pitchy; 
length 2.0-3.5 $\mathrm{mm}$

variabilis

3 (1). Dorsal surfaces not entirely reddish to bronzy red

Dorsal surfaces entirely reddish to bronzy red; length $3.5 \mathrm{~mm}$

badius

4 (3). Pronotum blackish brown, elytron piceous with 5 pairs of markings red-

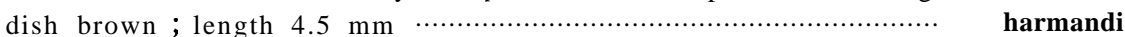

Dorsal surfaces reddish brown, elytron with a humeral, laterobasal and

a postmedian markings black; length $3.8-4.2 \mathrm{~mm}$

trimaculatus

Cleoporus badius Lefèvre (Fig. 33a)

Cleoporus badius Lef., 1889, Ann. Soc. Entomol. Fr., ser. 6,9: 294 (Cambodge : Promh-Penh) ; 1893, loc. cit. 1893 : 129 (Tonkin).

Cleoorus rugiceps Chen, 1940, Sinensia 11 (5-6) : 563 (S. China). -Gressitt \& Kimoto, 1961, Pac. Insects Monogr. 1A: 200 (S. China, Hainan, Tonkin). New synonymy.

Distribution: Thailand, Cambodia, Laos, Vietnam, Hainan, S. China.

The original description of Cleoporus badius Lefèvre is completely agreed to the characteristic Chinese species, C. rugiceps Chen.

Material examined. THAILAND: Bangkok, 25 exs., 2. vii. 1959, 1 ex., 30. xii. 1960 (BANGKEN) ; Chiangmai Prov., Fang (Agr. Expt. Station), 600 m, 1 ex., 14. vi. 1965, P. D. Ashlock (BISHOP). LAOS: Luang Prabang, 300 m, 1 ex., 4-5. vi. 1965, S. Quate (BISHOP). VIETNAM: Hoa-Binh,Tonkin, 3 exs., A. Cooman (FREY).

Cleoporus harmandi Lefèvre (Fig. 30b)

Cleoporus harmandi Le f., 1893, Ann. Soc. Entomol. Fr. 62: 129 (Cochinchina; PARIS).

DisTRIBUTION : Vietnam.

No additional material.

Cleoporus inornatus Jacoby (Fig. 31a)

Cleoporus inornatus Jac., 1908, Fauna India, Coleopt. 2: 480 (India: Bombay, Assam; Burma ; $\mathrm{BM})$.

Distribution : India, Burma, Thailand, Laos.

Material examined. THAilAnd: Chiangmai Prov., Fang (Agr. Expt. Station), 600 m, 1 ex., 14. vi. 1965, P. D. Ashlock; Tang Keo, 1 ex., 10 . vi. 1965, P. D. Ashlock; Mae Klang, 340 m, 4 exs., 11. vi. 1965, P. D. Ashlock (BISHOP) ; Mae Klang Water Fall, nr. Chom Thong, 3 exs., 11. vi. 1935, K. Morimoto \& Y. Miyatake ; Fang, 2 exs., 14. vi. 1965, K. Morimoto (KU). LAOS: Ban Van Heue, 20 km E of Phou-kow-kuei, 3 exs., 1-15. v. 1965, J. A. Rondon; Sedone Prov., Paksong, 2 exs., 17-18. v. 1965, P. D. Ashlock; Sedone Prov., Pakse, 1 ex., 15. v. 1965, P. D. Ashlock (BISHOP). VIETNAM : Dalat, 6 km S., 1400-1500 m, 1 ex., 9. vi.-7. vii. 1961, N. R. Spencer (BISHOP). 
Cleoporus trimaculatus Kimoto \& Gressitt, n. sp. (Fig. 31b)

Body oblong; dorsal surfaces reddish brown, pronotum with a pair of small, lateral markings blackish; elytron with humeral, postmedian and laterobasal markings blackish; head with vertex blackish, and labrum, clypeus and middle of vertex reddish brown; antenna dark reddish brown; ventral surfaces reddish brown with metathorax and part of basal segments black; legs reddish brown, femora with blackish marking apically.

Head with deep supra-ocular sulcus but without deep and wide excavation above eye ; surface somewhat rugose, strongly and closely punctate, and interstices of punctures much narrower than their diameters. Antenna slenderer, nearly half as long as body length; first segment robust, long, somewhat club-shaped ; second nearly half as long as first, robust ; third nearly twice as long as second, slender; fourth slightly shorter than third; fifth subequal to fourth in length and shape; sixth slightly shorter than fifth; seventh slightly longer than sixth; eighth nearly $11 / 3$ times as long as seventh; ninth and tenth subequal to eighth in length and shape; eleventh slightly longer than tenth and its apex pointed. Prontoum transverse, about $11 / 4$ times as wide as long, sides slightly rounded, widest almost at middle ; anterior and posterior corners each with a setigerous puncture; anterior margin slightly
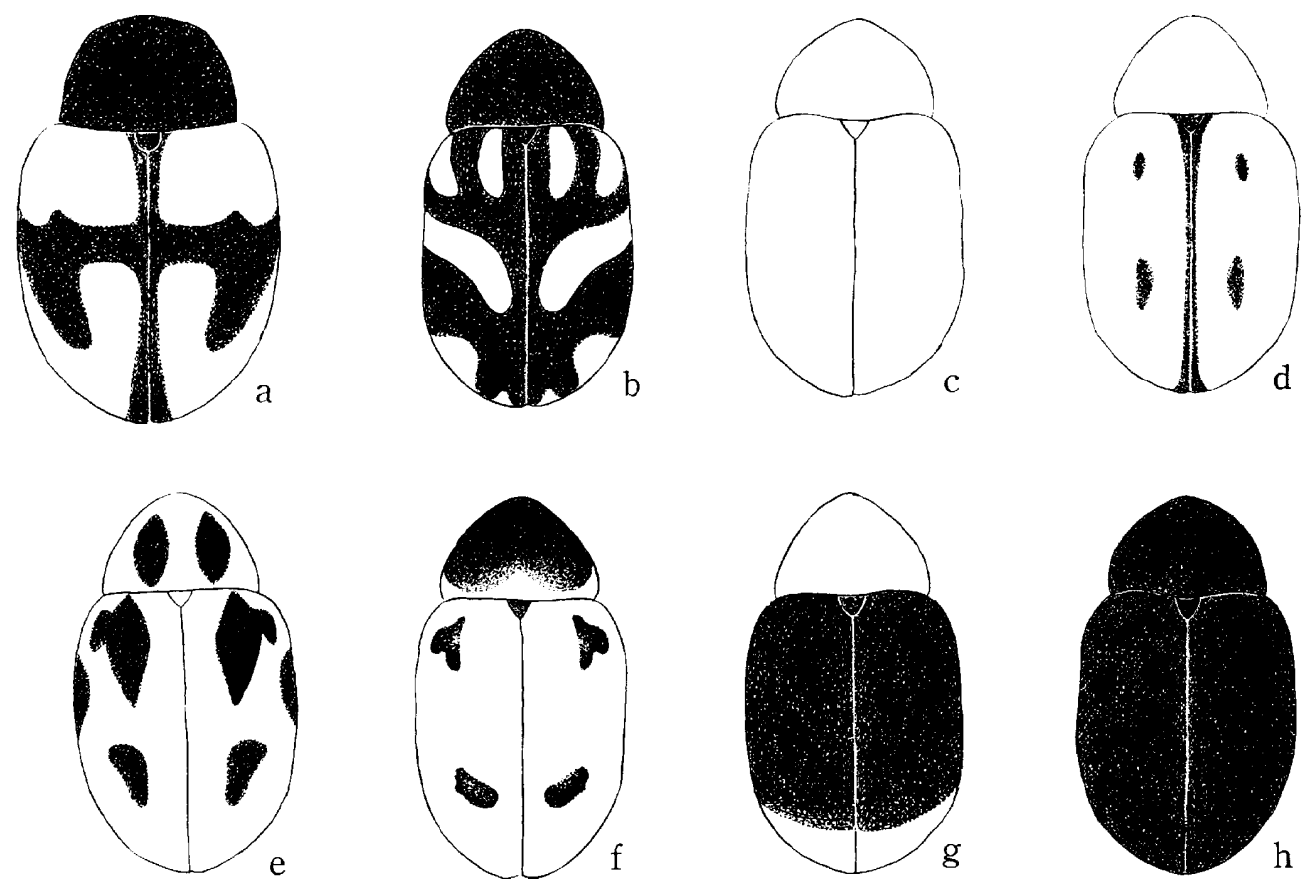

Fig. 30. a, Heterotrichus balyi Chapuis; b, Cleoporus harmandi Lefèvre; c-h, C. variabilis (Baly). 
rounded anteriorly and posterior margin more strongly so posteriorly; anterior sulcus distinct only on lateral portion; dorsal surface rather closely and distinctly punctate on lateral portion and sparsely and finely so medianly, and their interstices sparsely impressed by minute punctures ; proepimeron smooth, shining, nearly impunctate ; metasternum distinctly and rather closely covered with punctures and pubescence. Elytronsu bparallel-sided and rounded at apex; subbasal area slightly covex. and subbasal transverse furrow obsolete; punc. tures regularly arranged in longitudinal rows, and punctures larger and deeper on basal area and finer and shallower on apical area, and their interstices sparsely impressed by minute punctures and convex especially on lateral portion.

Length $3.8-4.2 \mathrm{~mm}$.

Holotype (BANGKHEN), THAILAND : Kanchanaburi, 12. х. 1935. Paratopotypes: 3 exs., same data as the holotype (BANGKHEN, BISHOP, KIMOTO).

This new species somewhat resembles C. badius Lefèvre, in having the head rugose on vertex, but differs in having metasternum distinctly and rather closely covered with punctures and pubescence, and the characteristic markings on the dorsal surfaces.

\section{Cleoporus variabilis (Baly) (Figs. 30c-h)}

Paria variabilis Baly, 1874, Trans. Entomol. Soc. Lond., 1874: 166 (E. Siberia, Japan ; BM). Paria robusta Baly, 1874, Trans. Entomol. Soc. Lond., 1874: 166 (Japan ;BM). -Chûjô \& Kimoto, 1961, Pac. Insects 3 (1) : 142 (=variabilis).

Stethotes tibialis Lefèvre, 1885, Ann. Soc. Entomol. Fr., ser. 6, 5: 65 (Tonkin), --Gressitt \& Kimoto, 1961, Pac. Insects Monogr. 1A: 201 (=variabilis).

Stethotes pallidipes Fairmaire, 1888, Ann. Soc. Entomol. Belg., 32: 36 (China). -Chen, 1935, Sinensia 6 (3) : 285 (=tibialis).

Mouhotina tibialis: Lefèvre, 1885, Ann, Soc. Entomol. Fr., ser. 6, 9: 293 (Cochinchina: Saigon ; Annam: Qui-Nhon ; Cambodia : Pnomh-Penh) ; 1893, Ann. Soc. Entomol. Fr., 1853: 128 (Tonkin).

Mouhotina rufipes Lefèvre, 1889, Ann. Soc. Entomol. Fr., ser. 6, 9: 293 (Cochinchina: Saigon ; Annam; Qui-Nhon ;Tonkin: Lien-Son) ; 1893, Ann. Soc. Entomol. Fr. 1893: 128 ( = pallidipes) .

Mouhotina pallidipes:Lefèvre, 1893, Ann. Soc. Entomol. Fr., 1893: 128 (Tonkin).

Cleoporus niger Weise, 1922, Tijdschr. Entomol. 65: 52 (Fukien; STOCKHOLM). --Gressitt \& Kimoto, 1961, Pac. Insects Monogr. 1A:201 (=variabilis).

Cleoporus pygmaeus Weise, 1922, Tijdschr. Entomol. 65: 53 (Fukien ; HAMBURG). - Gressitt \& Kimoto, 1961, Pac. Insects Monogr. 1A : 201 (=variabilis).

Cleoporus tibialis: Chen, 1935, Sinensia 6 (3) : 288 (China, Tonkin).

Cleoporus suturalis Chen, 1935, Sinensia 6 (3) : 287 (Tonkin; PARIS). -Gressitt\& Kimoto, 1961, Pac. Insects Monogr. 1A: 291 (Tonkin). New synonymy.

Cleoporus tibialis var. aeneipennis Chen, 1935, Sinensia 6 (3) : 286 (Tonkin).

Cleoporus variabilis: Chen, 1935, Sinensia 6 (3) : 288 (China). -.-Gressitt\& Kimoto, 1961, Pac. Insects Monogr. 1A: 201 (China, Hainan). -Kimoto, 1964, J. Fac. Agr. Kyushu Univ. 13 (2): 239 (Japan) ; 1972, Entomol. Ts. 93 (4): 145 (Annam).

Distriburion: E. Siberia, China, Korea, Japan, Taiwan, Hainan, Vietnam, 

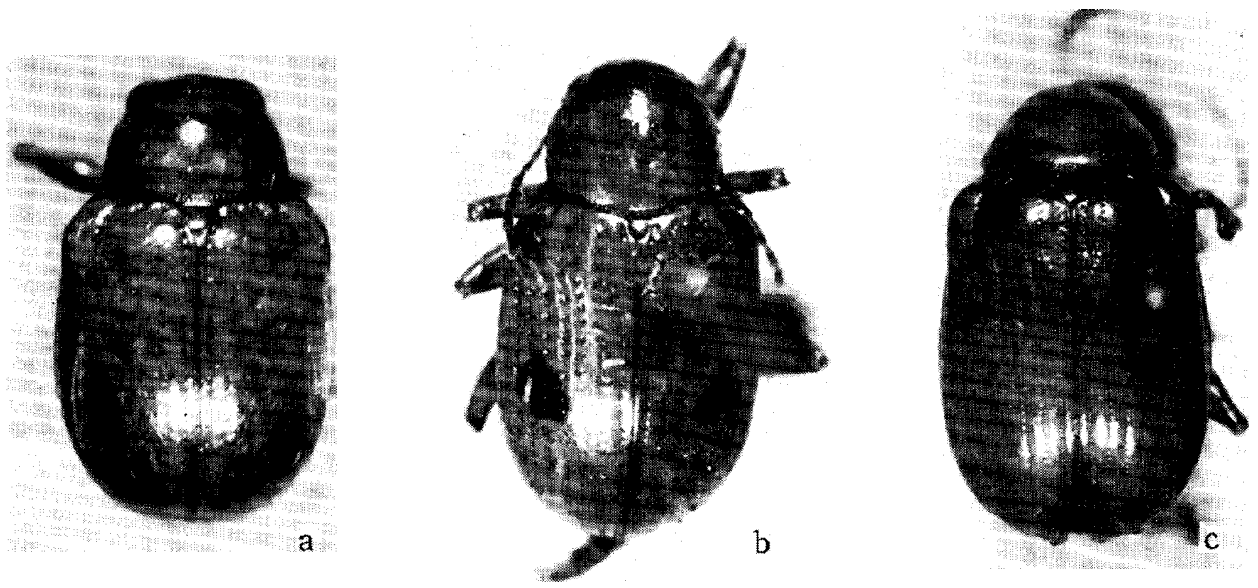

Fig. 31. a, Cleoporus inornatus Jacoby ; b, C. trimaculatus Kimoto \& Gressitt, n. sp.; c, Phytorus tonkinensis Lefèvre, cotype.

Laos, Cambodia, Thailand.

Material examined. THAilAND: Mae Klang Water Fall, nr. Chom Thong, 25 exs., 11. vi. 1965, K. Morimoto \& Y. Miyatake; Doi Suthep, $1000 \mathrm{~m}$, Chiang Mai Prov., 2 exs., 12. vi. 1965, K. Morimoto; Tan Keo, Suthep, 800 m, Chiang Mai Prov., 1 ex., 10. vi. 1965, Y. Miyatake; Fang, 28 exs., 13-14. vi. 1965, K. Morimoto; Chinag Mai, 3 exs., 9. vi. 1965, 3 exs., 12. vi. 1965, K. Morimoto ; Chieng Dao, 10 exs., 15. vi. 1965, K. Morimoto \& Y. Miyatake ; Botanical Garden, Chiang Mai, 1 ex., 1.2. vi. 1965, Y. Miyatake; Kao Yai Nat. Park, 30 exs., 556. vi. 1965, S. Asahina \& Y. Miyatake; Muak Lek, $40 \mathrm{~km}$ SE of Sara Buri, 6 exs., 5. vi. 1965, K. Morimoto; Pak Chong, nr. Kao Yai, 2 exs., viii. 1970, sorghum, K. Yano, 1 ex., 14. vi. 1973, Y. Yoshiyasu ; Mae Talai, nr. Chieng Dao, 4 exs., 3. vii. 1970, upland rice, K. Yano; Lampang, 1 ex., 16. vii. 1970, sweeping of paddy field, K. Yano (KU) ; Phu Kadueng, 1 ex., 7. x. 1972, M. Sato (EHIME) ; Chiangmai Prov., Chiangmai (Arboretum), 300 m, 2 exs., 12. vi. 1965, P. D. Ashlock; Chiang Mai Prov., Fang, 500 m, 4 exs., 13. vi. 1965, P. D. Ashlock; Chiang Mai Prov., Fang (Agr. Expt. Station), 600 m, 1 ex., 14. vi. 1965, P. D. Ashlock; Trang Prov., Khaophappha, Kaochong, $200 \mathrm{~m}, 2$ exs., 11-12. i. 1964, 200-400 m, 2 exs., 10-12. i. 1964, G. A. Samuelson; Saraburi Prov., Ban Muak Lek Nat. Park, 1 ex., 6. vi. 1965, P. D. Ashlock; Banna, Chawang, nr Nabon, 70 m, 1 ex., 5. ix. 1958, J. L. Gressitt (BISHOP) ; Songkhla, 20 exs., 14. vii. 1963 (BANGKHEN) . CAMBODIA: Ph. Chisau, $40 \mathrm{~km} \mathrm{~S}$ of P. Penh, $20 \mathrm{~m}, 2$ exs., 29. iv. 1961, N. R. Spencer (BISHOP). LAOS: Ban Van Heue, $20 \mathrm{~km}$ E of Phou-kow-kuei, 29 exs., 15-31. v. 1965, Native collr ; Vientiane Prov., Ban Van Eue, 1 ex., 31. ix. 1965, Native collr; Sedone Prov., Pakse, 1 ex., 14. v. 1965, 1 ex., 15. v. 1965, 3 exs., 17. v. 1965, P. D. Ashlock; Sedone Prov., $5 \mathrm{~km}$ E of Pakse, 1 ex., 13. v. 1965, 3 km N of Pakse, 1 ex., 23. v. 1965, P. D. Ashlock; Luang Prabang, $300 \mathrm{~m}$, 
1 ex., 4-5. vi. 1965, light trap, S. Quate \& L. W. Quate; Muong Sing, NW of Luang Prabang, 650 m, 6-10. vi. 1960, Melastoma, S. Quate \& L. W. Quate; Savanakhet Prov., Savanakhet, 1 ex., 5. vii. 1965, Native collr (BISHOP) ; Umgeb. Vientiane, 4 exs., ii-vi. 1963 (MUNCHEN). VIETNAM: Hoa-Binh, 20 exs., A. Cooman (FREY) ; Dalat, 1500 m, 2 exs., 26-27. ix. 1960, C. M. Yoshimoto, 1 ex., 28. iv.-4. v. 1960, L. W. Quate, 3 exs., 29. iv.-4. v. 1960, S. Quate \& L. W. Quate ; 6 km S., Dalat, 1400-1500 m, 9 exs., 9. vi.-7. vii. 1961, N. R. Spencer; $4 \mathrm{~km} \mathrm{~W}$ of Dalat, $1560 \mathrm{~m}$, Mtn., 1 ex., 4. v. 1960, R. E. Reech;DiLinh (Djiring) , 1200 m, 1 ex., 22-28. iv. 1960, S. Quate, 900 m, 1 ex.. 22-28. iv. 1960, L. W. Quate ; $7 \mathrm{~km}$ SE of Dilinh, 4 exs., 2. v. 1960, R. E. Leech; $9 \mathrm{~km} \mathrm{~S}$ of Dilinh, 1 ex., 24. iv. 1960, R. E. Leech ; $30 \mathrm{~km} \mathrm{NE} \mathrm{of} \mathrm{Dilinh,} 1$ ex., 27. iv. 1960, S. Quate \& L. W. Quate ; Mt. Lang Bian, 1500-2000 m, 4 exs., 19. v.-8. vi. 1961, N. R. Spencer; Ban Me Thuot, 500 m, 1 ex., 20-24. xii. 1960, C. M. Yoshimoto ; Dak Song, 76 km WE of Ban Me Thuot, 870 m, 1 ex., 19-21. v. 1960, L. W. Quate ; Karyu Danor, 200 m, 1 ex., 13-28. ii. 1961, N. R. Spencer; Kontum, N. of Pleiku, 550 m, 1 ex., 13. v. 1960, L. W. Quate (BISHOP).

\section{Genus Phytorus Jacoby}

Phytorus Jacoby, 1884, Ann. Mus. Civ. Genova 20: 226 (type : Phytorus dilatatus Jacoby ; Singapore). -Lefèvre, 1885, Mém. Soc. Sci. Liege, ser. 2, 11: 133. -Gressitt, 1955, Insects of Micronesia 17 (1) : 19.

\section{Phytorus tonkinensis Lefevre (Fig. 31c)}

Phytorus tonkinemis Le f., 1893, Ann. Soc. Entomol. Fr. 62: 128 (Tonkin; PARIS).

Distribution : Vietnam.

Suboblong ovate ; convex, elytron with regularly arranged longitudinal rows of punctures; general color reddish brown, with posterior part of elytron much paler; antenna blackish with basal segments brownish; legs entirely brownish ; length 6.0-6.5 mm.

No additional material.

\section{Genus Tricliona Lefevre}

Tricliona Lef., 1885, Bull. Soc. Entomol. Fr., ser. 6, 5: 147 (first species listed: Tricliona fasciata Lefevre, from Sumatra) ; 1885, Mém. Soc. Sci. Liege, ser. 2, 11: 141. -Jacoby, 1980, Fauna India, Coleopt. 2: 469 (type indicated as "T. bifasciata Lef." typographical error of T. fasciata Lef.) .-Chen, 1935, Sinensia 6 (3) : 289; 1940, ibid, 11 (5-6): 489. ---Gressitt \& Kimoto, 1961, Pac. Insects Monogr. IA: 202.

\section{KEY TO SPECIES OF Tricliona}

1 (0). Interocular area feebly punctured; pronotum very weakly punctured at side $\cdots 2$ Interocular area closely punctured; pronotum heavily and closely punctate at side 
2 (1). Elytron with puncture rows sulcate and interstices strongly raised to apex; dorsum reddish ; reddish ; length $5.5 \mathrm{~mm} \ldots \ldots \ldots \ldots$. costipennis

Elytron with puncture rows not sulcate and interstices not raised; reddish brown, elytron with lateral and apcial margins narrowly and sutural margin widely blackish; ventral surfaces with meso- and metathorax blackish; length 2.9-3.4 $\mathrm{mm}$

suturalis

3 (1). Head with longitudinal sulcus at middle shallow and shorter . . ....................4 Head with longitudinal sulcus at middle deep and distinct; length over

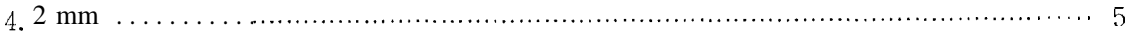

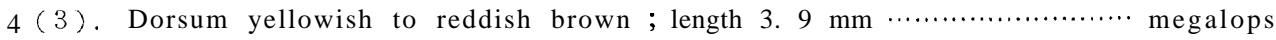
Dorsum entirely pitchy black, with slight aeneous reflextion, ventral surfaces pitchy brown; pitchy brown with basal segments paler ; legs dark

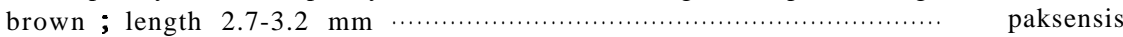

5 (3). Dorsum entirely pitchy black, with slight aeneous reflection, ventral surfaces pitchy brown; length $4.2-4.8 \mathrm{~mm} \ldots \ldots \ldots \ldots \ldots \ldots \ldots \ldots \ldots \ldots \ldots . . . . . . . \quad$ melanura

Dorsum pitchy, somewhat mixed with green ; elytron a broad longitudinal stripe, more deeply colored than lest of surface, extending from humerus to a littled:stance beyond middle ; ventral surfaces pitchy black ; length $4.5 \mathrm{~m} \mathrm{~m}$ consobrina

\section{Tricliona conso brina $\mathrm{C}$ hen}

Tricliona consobrina Chen, 1935, Sinensia 6: 291 (Tonkin).--Gressitt\& Kimoto, 1961, Pac. Insects Monogr. IA: 202 (Tonkin).

Distribution : Vietnam.

No additional material.

\section{Tricliona costipennis Chen}

Tricliona costipennis Chen, 1935, Sinensia 6: 292 (Tonkin; PARIS). -Gressitt \& Kimoto, 1961, Pac. Insects Monogr. 1A: 202 (Tonkin).

Distribution : Vietnam.

No additional material.

\section{Tricliona megalops Chen}

Tricliona megalops Chen, 1935, Sinensia 6: 294 (Tonkin; PARIS). -Gressitt \& Kimoto, 1961, Pac. Insects Monogr. 1A:202 (Tonkin).

Distribution : Vietnam.

No additional material.

Tricliona melanura Lefèvre (Fig. 32a)

Tricliona melanura Lef., 1890, Nouv. Arch. Mus. Paris, ser. 3, 2: 197 (Cambodge ; PARIS) ; 1904, Miss, Pavie Indo-China, ser. 2, 3 (Zool.): 153 (Cambodge).

Distribution : Cambodia, Laos, Vietnam.

Material examined. LAOS: Wapikhamthong Prov., Khong Sedone, 1 ex., 17. vii. 1965, Native collr; Sedone Pakse, 1 ex., 23. v. 1965. P. D. Ashlock (BISHOP) ; Umgeb. Vanky, 1 ex., 1963 (MUNCHEN). 

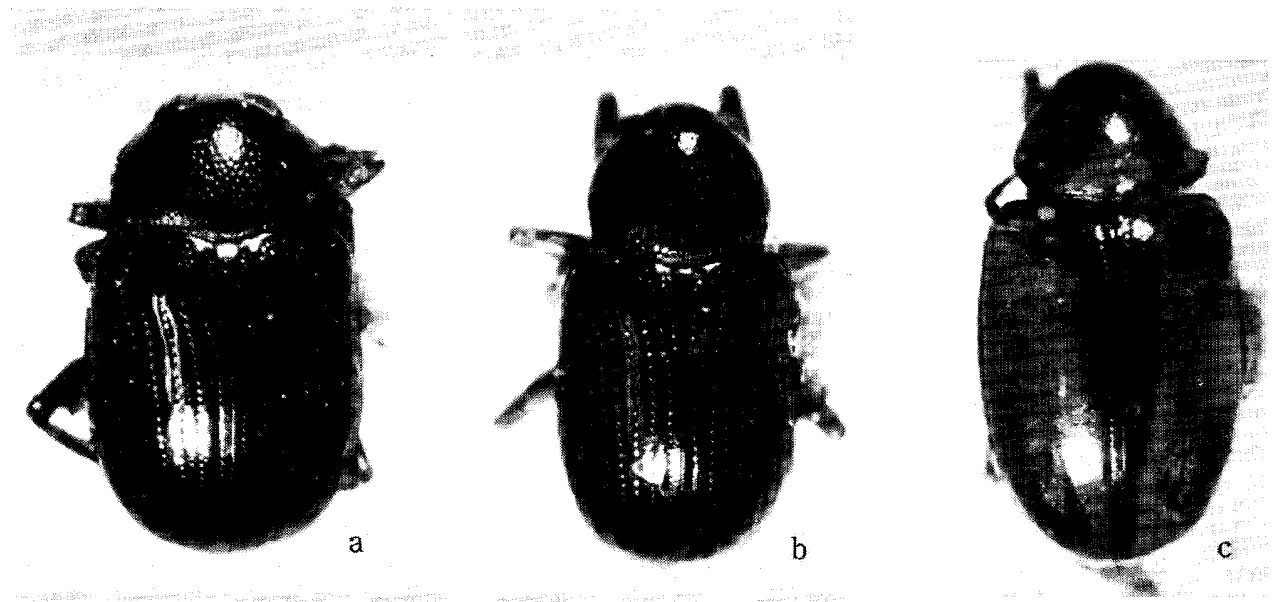

Fig. 32. a, Tricliona melanura Lefèvre; b, T. paksensis Kimoto\& Gressitt, n. sp.; c, T. suturalis Kimoto \& Gressitt, n.sp.

Tricliona paksensis Kitnoto \& Gressitt, n. sp. (Fig. 32b)

Oblong, subparallel-sided ; dorsum entirely pitchy black, with slight aeneous reflextion, ventral surfaces pitchy brown; antenna pitchy brown with basal segments paler; legs dark reddish brown.

Head with surface finely granulate, vertex sparsely impressed by distinct punctures, and with a shallow longitudinal furrow at middle; clypeus widely excavated at middle, separated from vertex by a distinct furrow. Antenna robust, nearly $2 / 3$ as long as body length; first segment robust, somewhat club-shaped; second nearly $2 / 3$ as long as first; third slightly longer than second, much slenderer; fourth and fifth subequal to third in length and shape; sixth as long as fifth but gradually widened apically; seventh as long as sixth but much robuster, subtriangular ; eighth slightly longer than seventh, robust; ninth slightly shorter than eighth, and nearly $11 / 2$ times as long as wide; tenth subequal to ninth in length and shape; eleventh nearly $13 / 4$ times as long as tenth and its apex pointed. Pronotum transverse, about $11 / 2$ times as wide as long, side slightly rounded, widest almost at middle and slightly narrowed anteriorly and posteriorly ; anterior and posterior corners slightly thickened, each with a setigerous puncture ; anterior margin rounded anteriorly and posterior margin more widely rounded posteriorly; dorsal surface distinctly and rather closely punctate and their interstices sparsely impressed by fine punctures; proepimeron finely granulate, nearly impunctate; metasternum smooth, shining, sparsely impressed by fine punctures and covered with fine hairs. Scutellum subpentagonal, with apex rounded; surface smooth, shining, nearly impunctate. Elytron subparallel-sided, with apex rounded; punctures large, distinct, regularly arranged in longitudinal rows, 
and their interstices sparsely impressed by fine punctures and slightly raised especially on lateral area. Femur of anterior leg with a distinct tooth sub. apically and femur of hind leg with a minute tooth subapically.

Length 2.7-3.2 $\mathrm{mm}$.

Hодотуре (BISHOP), LAOS: Sedone Prov., Pakse, 23. v. 1965, P. D. Ashlock. Paratype : LAOS: 3 km N of Pakse, 1 ex., 2. v. 1965, P. D. Ashlock (KIMOTO).

This new species somewhat resembles Tricliona melanura Lefèvre, but differs in being the body length shorter and having vertex of head more sparsley and finely punctate.

Tricliona suturalis Kimoto \& Gressitt, n. sp. (Fig. 32c)

Oblong oval; reddish brown, elytron with lateral and apical margins narrowly and sutural margin widely blackish; ventral surfaces with meso- and metathorax blackish.

Head with vertex smooth, shining, sparsely impressed by minute punctures, and with a shallow longitudinal furrow at middle; clypeus shagreened, flat, more sparsely impressed by stronger punctures than vertex. Antenna robust, nearly $2 / 3$ as long as body length ; first segment robust, somewhat club-shaped ; second nearly $3 / 4$ as long as first, robust; third nearly as long as second but much slenderer; fourth slightly longer than third, slender; fifth and sixth subequal to fourth in length and shape; seventh slightly longer than sixth, gradually widened apically, subtriangular ; eighth slightly longer than seventh and much robuster; ninth and tenth subequal to eighth in length and shape, and nearly twice as long as wide; eleventh $11 / 4$ times as long as tenth and its apex pointed. Pronotum transverse, about $11 / 4$ times as wide as long, side rounded, widest almost at middle and narrowed anteriorly and posteriorly; anterior and posterior corners each with a setigerous puncture ; dorsal surface sparsely impressed by fine punctures; proepimeron smooth, shining, impunctate; metasternum smooth, shining, sparsely impressed by fine punctures and fine hairs. Elytron subparallel-sided, with apex rounded; punctures distinct on basal part and obsolete on apical part, regularly arranged in longitudinal rows and their interstices flat, sparsely impressed by minute punctures. Femur of anterior leg armed with a distinct tooth subapically and femur of posterior leg with a minute tooth subapically.

Length 2.9-3.4 $\mathrm{mm}$.

Hоготуре: Chiang Dao, (Type No. 2359, Kyushu Univ.), 15. vi. 1965, K. Morimoto. Paratopotypes : THAILAND : 2 exs., same data as the holotype (KU). Paratypes : THAILAND : Botanical garden, Chiangmai, 1 ex., 12. vi. 1965, Y. Miyatake (KU) ; Chiang Dao, 4 exs., 15. vi. 1965, P. D. Ashlock (BISHOP).

This new species somewhat resembles Tricliona marginata Jacoby, from India, but differs in being the body length shorter, and having prothorax narrower and elytral suture widely blackish. 


\section{Genus Cleorina Lefèvre}

Cleorina Lef., 1885, Mém.Soc. Sci. Liège, ser. 2, 11 : 143. Jacoby, 1908, Fauna India, Coloept. 2: 432 (type designated : Cleorina aulica Lefèvre= Nodostoma aeneomicans Baly, from Malacca) --Chen, 1935, Sinensia 6 (3) : 281; 1940, ibid. 11 (5-6) : 488. -Chûjô, 1956, Philip. J. Sci. 85 (1) : 148. Gressitt \& Kimoto, 1961, Pac. Insects Monogr. IA: 202.

\section{Key to SPECIES of Cleorina}

1 (0). Legs entirely blackish

Legs partly reddish; blackish blue, antenna with basal segments reddish; length $3.0 \mathrm{~mm}$.

2 (1). Proepimeron not distinctly punctured, surface finely granulate ; metasternum heavily punctured

Proepimeron distinctly punctured, surface smooth, shining ; metasternum nearly impunctate, except for a few marginal ones; coloration of dorsal surfaces variable: entirely greenish, violaceous, bluish or blackish, in some specimen ground color green with disc of elytron cupreous; length $3.0-4.2 \mathrm{~mm}$

aeneomicans Of large size; entirely blackish blue ; length 4.0-4. $5 \mathrm{~mm}$.................... major

Of small size; entirely blackish with or without slightly bluish luster; length 2. $5-3.0 \mathrm{~mm}$

bainana

\section{Cleorina aeneomicans (Baly)}

Nadostoma aeneomicans Baly, 1867, Trans. Entomol. Soc. Lond., ser. 3, 4 (2) : 237 (Malacca; BM). Jacoby, 1884, Notes Leyd. Mus. 6: 15 (Sumatra) ; 1889, Ann. Mus. Civ. Genova 1889: 165 (Burma).

Nodostoma purpureipenne Baly, 1867, Trans. Entomol. Soc.Lond., 3, 4 (2) : 249 (Malacca ; BM), $\mathrm{N}$ ew synonymy.

Cleorina aeneomicans: Lefèvre, 1887, Notes Leyd. Mus. 9: 263 (synonymies) ; 1889, Ann, Soc. Entomol. Fr. ser. 6, 9: 293 (Cambodge: Pnomh-Penh). -Jacoby, 1889, Ann. Mus. Civ. Genova 1885: 165 (Burma) ; 1896, ibid. 36: 412 (Sumatra). -Lefèvre, 1893, Ann. Soc. Entomol. Fr. 1893: 129 (Env. Bangkhok).

Cleorina aulica Lefèvre, 1885, Mém. Soc. Sci. Liège, ser. 2, 11: 144, nota 1 (Batavia) ; 1887, Notes Leyd. Mus. 9: 263 (=aeneomicans).--Jacoby, 1908, Fauna India, Coleopt. 2: 488 (Java, Burma).

Cleorina janthina Lefèvre, 1885, Mém. Soc. Sci. Liège, ser. 2, 11: 144, nota 3 (Hongkong). Chen, 1935, Sinensia 6 (3): 282 (Hongkong). ---Gressitt \& Kimoto, 1961, Pac. Insects Monogr. 1A : 203 (S. China. China, Hainan Is.). N ew synonymy.

Cleorinalefevrei Jacoby, 1890, Entomologist $23: 116$ (China ; BM) ; 1908, Fauna India, Coleopt. 2: 484 (Burma, China). -Chen, 1935, Sinensia 6 (3) : 282. Chû jô, 1956, Philip. J. Sci. 85 (1) : 150, fig. 12 (Formosa), --Gressitt \& Kimoto, 1961, Pac, Insects Monogr. 1A: 203 (=janthina).

Cleorina purpureipennis: Jacoby, 1896, Ann. Mus. Civ. Genova 36: 414 (genus).

Cleorina purpureipennis varr. purpureicollis, violaceipennis Pic, 1937, Mél. Exot. Entomol. 69: 23 (Tonkin).

Distribution : Burma, Andaman Is., Thailand, Cambodia, Laos, Vietnam, Hainan, S. China, Taiwan, Malaya, Sumatra, Java.

Material EXAmined. THAILAND: Doi Suthep, 1000 m, Chiang Mai Prov., 1 ex., 8. vi. 1965, K. Morimoto, 3 exs., 18-19. vi. 1965, K. Morimoto \& Y. Miyatake ; 

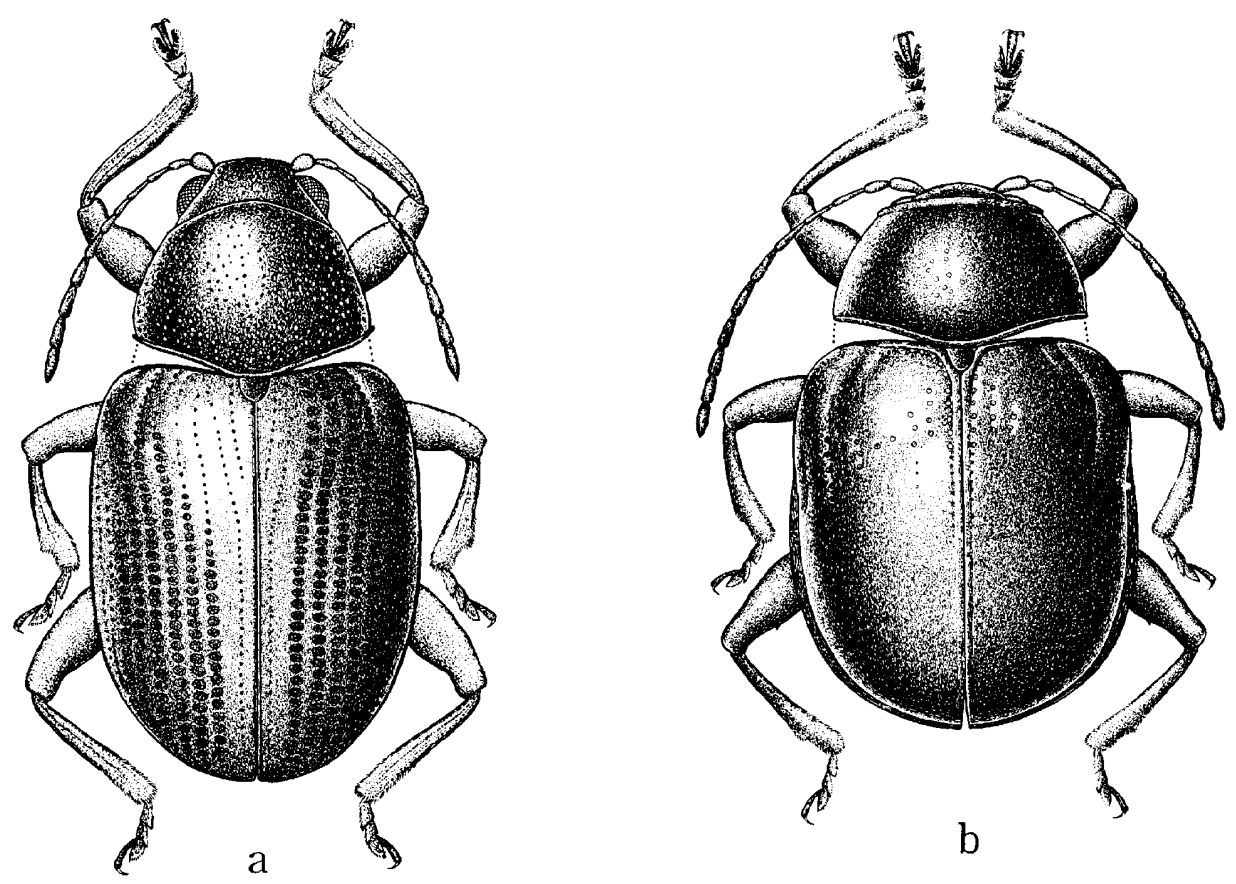

Fig. 33. a, Cleoporus badius Lefèvre ; b, Cleorina hainana Gressitt \& Ki moto.

Khao Yai Nat. Park, 2 exs., 6. vi 1965, K. Morimoto; Khao Chong, nr. 'Trung, 26. vi. 1965, Y. Miyatake (KU) ; Trang Prov., Khaophappha, Kaochang, 200-400 m, 1 ex., 31. i. 1964, 3 exs., 11-12. i. 1964, G. A. Samuelson; Nakhon Nayok Prov., Khao Yai Nat. Park, 1 ex., 6. vi. 1965, P. D. Ashlock; Banna, Chawang, nr. Nabon, 70 m, 1 ex., 5. ix. 1958, J. L. Gressitt ; Chinagmai Prov., Chiang Dao, 450 m, 1 ex., 5-11. iv. 1958, T. C. Maa (BISHOP). LAOS: Vientiane Prov., Ban Van Eue, 1 ex., 15. 31. v. 1965, 1 ex., 31. vii. 1965, Native collr ; Ban Van Heue, 20 km E of Phou-kow-kuei, 5 exs., 15-31. v. 1965, Native collr; Borikhane Prov., Pakkading, 1 ex., 31. vii. 1965, 1 ex., 3. viii. 1965, Native collr ; Borikhane Prov., Paksane, 1 ex., 3. xii. 1965, Native collr; Sedone Prov., Paksong, 1 ex., 1. vi. 1965, Native collr ; Attopeu., Houei Kong, 2 exs., 31. v. 1965, Native collr (BISHOP) ; Umgeb. Vientiane, 3 exs., iii-vi. 1963; Umgeb. Paklay, 1 ex., 1963 (MUNCHEN). VIETNAM: Fyan, 900-1000 m, 8 exs., 11. vii.-9. viii. 1961, N. R. Spencer; $18 \mathrm{~km} \mathrm{NW}$ of Dalat, $1300 \mathrm{~m}, 1$ ex., 4-5. v. 1960, L. W. Quate ; Blao (Balao), $500 \mathrm{~m}, 1$ ex., 22. iv. 1960, 5 exs., 14-21. x. 1960, C. M. Yoshimoto (BISHOP) ; Hoa-Binh, Tonkin, 20 exs., A. Cooman (FREY).

Cleorina bicoloripes Pic

Cleorina bicoloripesPic, 1937, Mél.Exot. Entomol. 69: 23 (Tonkin).

DISTRIBUTION: Vietnam. 
We could not trace the type location of this species. No additional material.

Cleorina hainana Gressitt \& Kimoto (Fig. 33b)

Cleorina hainana Gressitt \& Kimoto, 1961, Pac. Insects Monogr. 1A: 203 (Hainan I.;CAS).

Distribution: Thailand, Laos, Vietnam. Hainan.

Material examined. THAILAND : Trang Prov., Kahophappha, Kaochong, 200-400 m, 10. i. 1964, G. A. Samuelson (BISHOP). LAOS: Ban Van Heue, $20 \mathrm{~km}$ E of Phou-kow-kuei, 2 exs., 15-31. v. 1965, J. A. Rondon, 1 ex., 1-15. v. 1965, Malaise trap, J. A. Rondon; Ile de Khong, 1 ex., 6. ii. 1965, 1 ex., 7. ix. 1965, Native collr (BISHOP) ; Vientiane, 2 exs., iii-vi. 1963 (MUNCHEN). VIETNAM: Fyan, 900-1000 m, 5 exs., 11. vii.-9. viii. 1961, N. R. Spencer ; Mt. Lang Bian, 1500-2000 m, 4 exs., 19. v.-8. vi. 1961, N. R. Spencer; Dalat, $1500 \mathrm{~m}$, 3 exs., 29. iv.-4. v. 1960, S. Quate \& L. W. Quate; 6 km S., Dalat, 1400-1500 m, 4 exs., 9. vi.--7. vii. 1961, N. R. Spencer; M'Drak, E of Ban Me Thuot, 4-600 m, 2 exs., 8-19. xii. 1960, C. M. Yoshimoto; Dai Lanh, $\mathrm{N}$ of Nhang, Trang, 3 exs., 30. xi.-5. xii. 1960, C. M. Yoshimoto; $22 \mathrm{~km} \mathrm{~S}$ of Nha Trang, 1 ex., 20-26. xi. 1960, C. M. Yoshimoto (BISHOP).

\section{Cleorina major Kimoto \& Gressitt, n. sp. (Fig. 34a)}

Body very round, convex; entirely blackich blue; antenna blackish with basal segments partly brownish.

Head with surface strongly and closely punctate, vertex with a shallow longitudinal furrow at middle. Antenna filiform, nearly $2 / 3$ as long as body length; first segment robust, somewhat club-shaped ; second robust, nearly half as long as wide, and almost $2 / 3$ as long as first; third slender, nearly 1 $1 / 2$ times as long as second ; fourth nearly $11 / 5$ times as long as third; fifth subequal to fourth in length but robuster ; sixth subequal to fifth in length and shape; seventh slightly shorter than sixth; eighth to tenth subequal to seventh in length and shape; eleventh slightly longer than tenth and its apex pointed. Pronotum transverse, about $12 / 3$ times as wide as long, side sligthly rounded, widest at basal margin and narrowed anteriorly; anterior and posterior corners each with a setigerous puncture; anterior margin nearly straight and posterior margin rounded and distinctly produced posteriorly at middle; anterior sulcus distinct, deep and impressed by a row of deep punctures; dorsal surface closely impressed by large punctures which are larger than average elytral punctures_ and interstices of punctures subequal or narrower than diameter of punctures and distinctly raised longitudinally on lateral portion ; proepimeron finely granulate, impunctate ; metasternum closely impressed by large punctures, and their interstices partly granulate. Scutellum subpentagonal, convex ; surface finely and rather closely punctate at middle. 
Elytron with side rounded, widest slightly behind humerus and gradually narrowed and apex rounded; subbasal area distinctly raised, separated from behind by a distinct subbasal transverse furrow ; punctures regularly arranged in longitudinal rows and their interstices finely impressed by minute punctures and distinctly raised especially on lateral portion.

Length $4.0-4.5 \mathrm{~mm}$.

Holotype (BISHOP), LAOS: Ban Van Heue, $20 \mathrm{~km}$ E of Phou-kow-kuei, 1531. v. 1965, J. A. Rondon. Paratopotypes: 2 exs., same data as the holotype (BISHOP, KIMOTO). Paratypes : LAOS: 2 exs., same data as the holotype but 15. v. 1965, J. A. Rondon (BISHOP); Vientiane Prov., Ban Van Heue, 1 ex., 15. v. 1965, 1 ex., 15-31. v. 1965, Native collr (BISHOP) ; Umgeb. Vientiane, 1 ex., iii-vi. 1963 (MUNCHEN).

This new species somewhat resembles C. aeneomicans (Baly), but differs in having proepimeron impunctate and finely granulate and metasternum closely impressed by large punctures.

\section{Genus Mouhotina Lefèvre}

M ouhotia Baly, 1878 (nec Castelnau, 1862), J. Linn. Soc. Zool. 14: 262 (type : M ouhotia femorata Baly, from Cambodia).

M ouhotina Lefèvre, 1885, Mém. Soc. Sci. Liège, ser. 2, 11: 130 (new name for Mouhotia Baly, 1878, nec Castelnau, 1862). -Jacoby, 1908, Fauna India, Colept. 2: 490.

\section{Key to species of Mouhotina}

Body length longer; pronotum somewhat finely punctate; reddish brown, head, thorax, antenna and legs mostly black, ventral surfaces of thorax pitchy black, in most pale colored specimen reddish brown with antenna and legs mostly pitchy black and in most dark colored specimen pitchy black with part

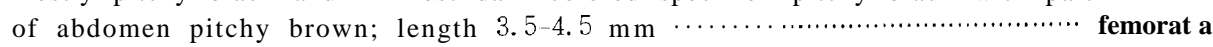

Body length shorter; pronotum nearly impunctate; shining black; antenna reddish brown with apical segments much darker; legs dark reddish brown to pitchy black ; length $2.4-2.6 \mathrm{~mm}$

minuta

Mouhotina femorata (Baly) (Fig. 34b)

M ouhotia femorata Baly, 1878, J. Linn. Soc. Lond., 14: 262 (Cambodia; BM).

M ouhotina femorata: Lefèvre, 1885, Mém. Soc. Sci. Liège, ser. 2, 11: 130 (Cambodge).

Distribution: Thailand, Cambodia, Vietnam.

Material examined. THAiland: Khao Chong, nr. Trung, 1 ex., 27. vi. 1965, Y. Miyatake (KU). VIETNAM: $9 \mathrm{~km} \mathrm{~S}$ of Dilinh (Djiring), 4 exs., 24. iv. 1960, R. E. Leech; $40 \mathrm{~km} \mathrm{~N}$ of Dilinh, $540 \mathrm{~m}, 2$ exs., 26. iv. 1960, R. E. Leech $28 \mathrm{~km} \mathrm{~N}$ of Dilinh, $900 \mathrm{~m}, 1$ ex., 22-28. iv. 1960, L. W. Quate (BISHOP).

Mouhotina minuta Kimoto \& Gressitt, n. sp. (Fig. 34c)

Ovate, convex ; shining black, antenna reddish brown with apical segments 
much darker; legs dark reddish brown to pitchy black.

Head with surface finely and not closely punctate; vertex convex, with a short shallow longitudinal furrow at middle; anterior margin of clypeus angularly notched. Antenna robuster, nearly $2 / 3$ as long as body length; apical seven segments compressed; first segment robust, somewhat club-shaped ; second nearly $3 / 4$ as long as first, rubust; third nearly as long as second, slender; fourth subequal to third in length, but slightly robuster; fifth nearly $11 / 4$ times as long as fourth, slightly widened apically; sixth and seventh subequal to fifth in length and shape; eighth sligthly shorter than seventh, and nearly twice as long as wide; ninth slightly shorter than eighth; tenth subequal to tenth in length and shape; eleventh slightly longer than tenth and its apex pointed. Pronotum transverse, nearly twice as wide as long; side slightly rounded, widest at basal margin and gradually narrowed anteriorly; anterior and posterior corners each with a setigerous puncture; anterior margin nearly straight and posterior margin rounded posteriorly and slightly produced at middle; anterior sulcus distinct, deep and impressed by a row of distinct punctures; dorsal surface nearly impunctate; proepimeron smooth, nearly impunctate; metasternum slightly wrinkled, sparsely impressed by minute punctures. Scutellum subtrigonate, with apex rounded, convex ; surface smooth, shining, impunctate. Elytron rounded at side, widest slightly behind humerus and gradually narrowed posteriorly and apex rounded ; subbasal area distinctly raised, separated from behind by a distinct subbasal transverse furrow; punctures regularly arranged in longitudinal rows, and punctures deep and distinct on basal area, and nearly obsolete on apical area, and their interstices distinctly impressed by minute punctures; humerus distinctly raised, and with a distinctly raised costa starting from humerus.

Length $2.4-2.6 \mathrm{~mm}$.

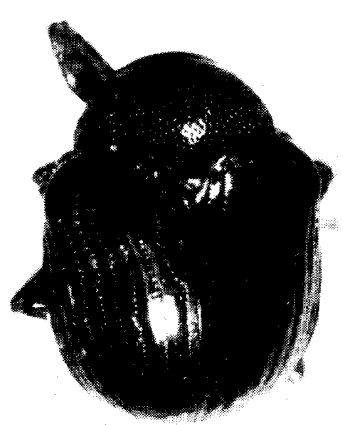

a
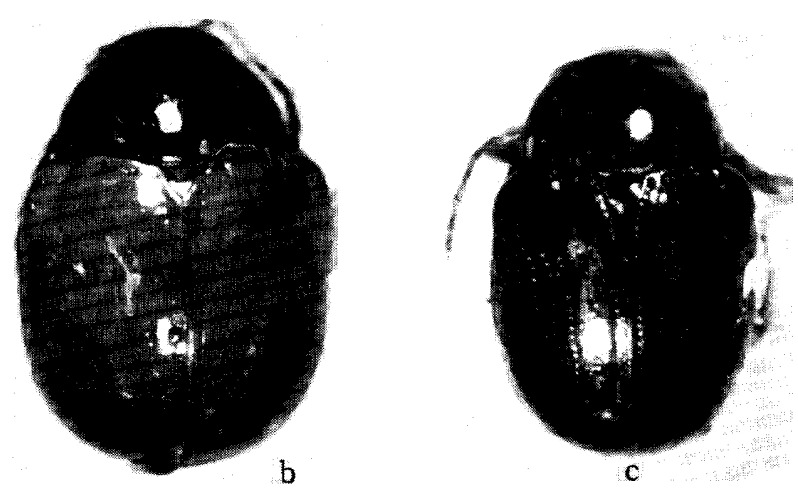

Fig. 34. a, Cleorina major Kimoto \& Gressitt, n. sp.; b, Mouhotina femorata(Baly); c, M. minuta Kimoto \& Gressitt, n. sp. 
Holotype (Type No. 2360, Kyushu Univ .), THAILAND : Chiang Mai Prov., Doi Suthep, 1000 m, 10. vi. 1965, K. Morimoto. Paratopotypes: 13 exs., same data as the holotype (KU, KIMOTO). Paratypes : THAILAND: 1 ex., same locality but 8 vi. 1965, S. Asahina; Chiangmai Prov., Doi Pui, 1300 m, 2 exs., 17. vi. 1965, K. Morimoto; Tankeo, Doi Suthep, 800 m, 10 exs., 10. vi. 1965, Y. Miyatake (KU) ; Doi Suthep, 1300 m, 2 exs., 8. vi. 1965, P. D. Ashloock; Doi Pui, 2 exs., 17. vi. 1965, P. D. Ashlock; VIETNAM: Dalat, 1500 m, 1 ex., 29. iv. 1960, L. W. Quate; $18 \mathrm{~km} \mathrm{NW}$ of Dalat, $1300 \mathrm{~m}, 2$ exs., 4-5. v. 1960, S. Quate \& L. W. Quate (BISHOP).

This new species somewhat resembles M. femorata(Baly), but differs in being the body length shorter, and ha\&g pronotum nearly impunctate.

\section{Genus Chalcolema Jacoby}

Chalcolema Jacoby, 1830, Entomologist 23: 84 (type : Chalcolema foveicollis Jacoby, from China). --Chen, 1935, Sinensia 6 (3) : 241; 1940, ibid. 11 (5-6) : 492. -Gressitt \& Kimoto, 1961, Pac. Insects Monogr. 1A: 276.

Tillopsis Berlioz, 1917, Ann. Soc. Entomol. Fr. 86: 312 (type: Tillopsis angustata Berlioz, from Laos). N ew synonymy.

Laoseumolpus Pic, 1935, Mél. Exot. Entomol. 75: 3 (type: Laoseumolpus bicolor Pic, from Laos). N ew synonymy.

Abirellus Chûjô, 1956, Philip. J. Sci. 85 (1) : 133 (type : Chrysochus cylindricus Chûjô, f ro m Taiwan).

$$
\text { Key to species of Chalcolema }
$$

Entirely blackish blue; antenna black with basal segments brownish; length 7.0

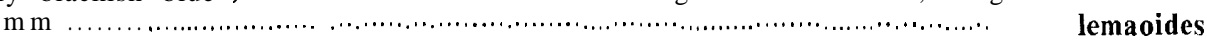

Reddish brown, elytron bluish black or bluish green; antenna pitchy black with

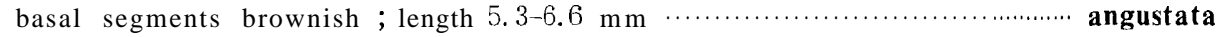

Chalcolema angustata (Berlioz) New combination (Fig. 35a)

Tillopsis angustata Berlioz, 1917, Ann. Soc. Entomol. Fr., 86: 313 (Laos ; PARIS).

Laoseumolpus bicolor Pic, 1935, Mel. Exot. Entomol. 65: 3 (Laos ; PARIS). N ew synonymy.

Distribution : Laos, Vietnam.

Material examined. LAOS: Vientiane Prov., Ban Van Eue, 4 exs., 15. v. 1965, Native collr; Muong Sing, NW of Luang Prabang, 650 m, 1 ex., 6-10. vi. 1960, L. W. Quate (BISHOP). VIETNAM: Ban Me Thuot, $500 \mathrm{~m}, 2$ exs., 16-18. v. 1960, L. W. Quate (BISHOP) ; Hoa-Binh, Tonkin, 1 ex., A. Cooman (FREY).

\section{Chalcolema lemaoides (Pic) New combination}

Tillopsis lemaoides Pic, 1928, Mel. Exot. Entomol. 52: 28 (Tonkin:Chapa; PARIS).

Distribution: Vietnam.

No additional material. 


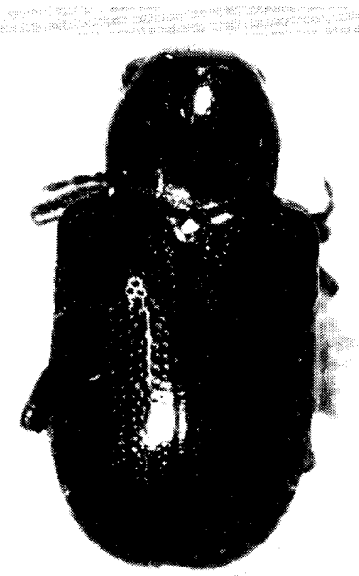

a
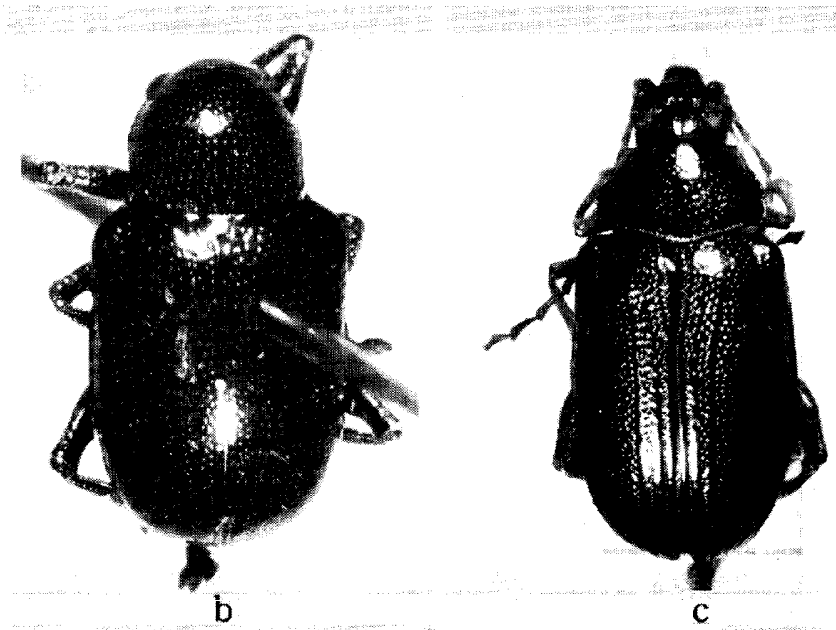

Fig. 35. a, Chalcolema angiustata (Berlioz); b, Heminodes an tennatus (Jacoby) ; c, Olorus speciosus Berlioz.

\section{Gemus Heminodes Jacoby}

Heminodes Jac., 1895, Ann. Soc. Entomol. Belg. 39: 287 (type : Heminodes indicus Jacoby, from India) ; 1908, Fauna India, Coleopt. 2: 512.

\section{Heminodes antennatus (Jacoby) (Fig. 35b)}

A birus antennatus Jac., 1892, Ann. Mus. Civ. Genova 32: 912 (Burma ; GENOVA).

Henzinodes antennatus: Jacoby, 1908, Fau na India, Coleopt. 2514 (Burma).

Distribution: Burma, Laos.

Oblong-elongate, glabrous above ; antenna with apical five segments broadly dilated; pronotum strongly and rather closely punctate; elytron with punctures arranged in semiregular rows; dorsum dark aeneous ; bluish black or violaceous blue; beneath metallic greenish, bluish or violaceous; length 6.0$10.0 \mathrm{~mm}$.

Material examined. LAOS: Khammouane Prov., Phon Tiou, 1 ex., 30. iv. 1965, Native collr; Hrikhane Prov., Pakkading, 2 exs., 22. iii. 1965, Native collr (BISHOP) ; Umgeb. Vientiane, 2 exs., iii-vi. 1963 (MUNCHEN).

\section{Genus Olorus Chapuis}

Olorus Chapuis, 1874, Genera Coleopt. 10: 311 (type: Olorus femoralis Chapuis, from Siam). Lefèvre, 1885, Mkm. Soc. Sci. Liège, ser. 2, 11: 115.

\section{KEY TO SPECIES OF Olorus}

Male: general color yellowsih brown; head and pronotum dark brown with greenish luster; elytron dark reddish brown; length $7 \mathrm{~mm}$ femoralis 
Male: head, pronotum, scutellum and elytron golden green, ventral surfaces and legs entirely reddish brown, posterior femur with a long projection subapically golden green or blackish blue, with ventral surfaces and legs slightly brownish in part, posterior femora with a distinct spine subapically; antenna blackish with basal segments brownish; length 7. 8-8. $3 \mathrm{~mm}$

speciosus

\section{Olorus femoralis Chapuis}

Olorus femoralis Chap., 1874, Genera Coleopt. 10: 312, nota 1, pl. 121, fig. 1 ("Natal": Juthia), -Lefèvre, 1885, Mém.Soc. Sci. Liège, ser. 2, 11: 116 ("Siam”).

Distribution : Thailand.

No additional material examined.

\section{Olorus speciosus Berlioz (Fig. 35c)}

Olorus speciosus Berlioz, 1917, Ann. Soc. Entomol. Fr., 86: 311 (Laos; PARIS).

Distribution : Thailand, Laos.

Material EXAmined. THAILAND: NW Chiangmai, Fang, 500 m, 1 ex.. 1219. iv. 1958, T. C. Maa (BISHOP). LAOS: Ban Van Heue, $20 \mathrm{~km}$ E of Phou-kowkuei, 1 ex., 1-5. v. 1965, J. A. Rondon (BISHOP) ; Umgeb. Vientiane, 4 exs., iiivi. 1963 ; Umgeb. Vanky, 1 ex.. 1963; Umgeb. Paklay, 1 ex., 1963 (MUNCHEN).

\section{Genus Colaspoides Castelnau}

Colaspoides Castelnau, 1883, in Silbermann, Rev. d'Entomol. 1: 20 (type :Cryptocephalus limbatus Fabricius, from Pueruto Rico). - Chapuis, 1874, Genera Coleopt. 10: 346. --Lefèvre, 1883, Mém. Soc. Sci. Liège, ser. 2, 11: 157. -Jacoby, 1908, Fauna India, Coleopt. 2: 514. Chen, 1935, Sinensia 6 (3) : 249; 1940, ibid. 11 (5-6) : 492. -Chûjô, 1956, Philip. J. Sci. 85 (1) : 160. --Gressitt \& Kimoto, 1961, Pac. Insects Monogr. IA : 276. -Kimoto, 1964, J. Fac. Agr. Kyushu Univ. 13 (2) : 239.

Amasia Chapuis, 1874, Genera Coleopt. $10: 313$ (type :Amasiaspinipes Chapuis =Colaspoides varians Baly, from Java).

Tailandia Chûjô, 1964, Nature and Life in SE Asia, Kyoto 3:281 (type : Tailandia chakratongii Chûjô, from Thailand). New synonymy.

\section{KEY TO SPECIES OF Colaspoides}

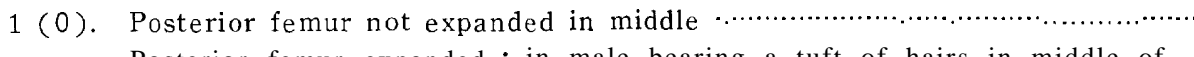

Posterior femur expanded; in male bearing a tuft of hairs in middle of posterior femur; male green, female blue; legs pitchy black, antenna pitchy black with basal four or five segments brownish ; length 5.1-5.7 $\mathrm{mm}$ femoralis

2 (1). Posterior femur with a distinct spine and anterior femur widened, angu-

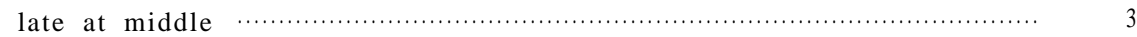

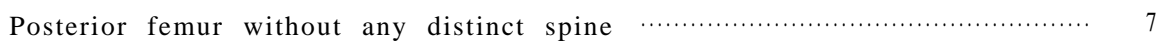

3 (2). Elytron largely not rugose, without distinct longitudinal costa dorsally $\cdots \cdots \cdots \cdots, 4$ Elytron largely subrugose, and with three or four longitudinal costae dorsally; dorsal surfaces generally cupreous with slight greenish luster; ventral surfaces pitchy brown with or without greenish luster; legs 
dark brown; length 6.0-6.3 $\mathrm{mm}$

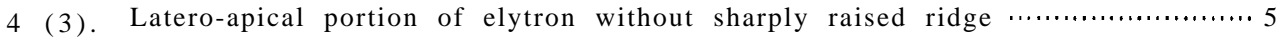

Latero-apical portion of elytron with a sharply raised ridge; ventral surfaces blackish with or without greenish to bluish luster; dorsal surfaces golden green, cupreous, violaceous or bluish black ; legs bluish black, greenish brown or reddish brown; length 4. 3-6. $1 \mathrm{~mm} \ldots \ldots \ldots \ldots \ldots$ prasinus

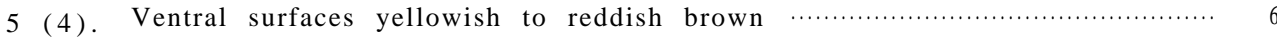

Ventral surfaces entirely pitchy black; general color green with or without metallic luster; antenna reddish brown with apical segments in part or largely blackish ; legs reddish brown; length 3.5-4. $6 \mathrm{~mm} \cdots \cdot$ thailandicus

6 (5). Posterior femur armed with a long and sharp spine subapically; dorsal surfaces greenish cupreous; length $4.5 \mathrm{~mm}$............................ spinigerus

Posterior femur armed with a broad spine subapically; metallic green to

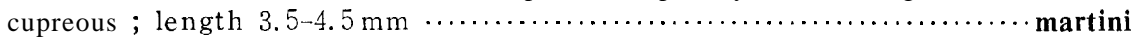

7 (2). Elytral surface largely subrugose, closely impressed by large punctures,

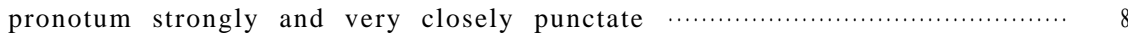

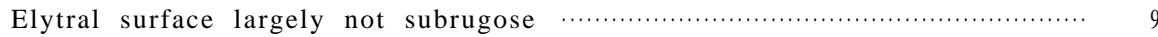

8 (7). Short oval, elytron with three or four longitudinal costae, golden green to greenish blue; antenna pitchy black with basal segments brownish;

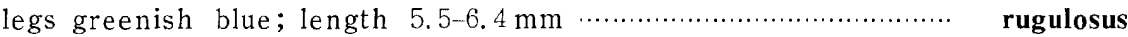

Oblong oval, elytron with irregular transverse or tubercular rugosities, all over surface; golden green, antenna ochraceous ; legs golden green;

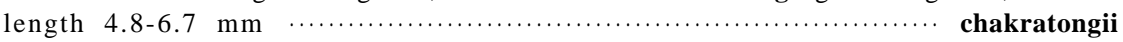

9 (7). Antenna robuster, preapical segments nearly twice as long as wide; head with vertex finely granulate especially on anterior portion $\ldots . . . . . . . . . . . .610$

Antenna slenderer, preapical segments much longer than twice as long as wide

10 (9). Prontoum distinctly and fairly closely punctate throughout and interstices of punctures closely impressed by minute puntures; bronzy black, antenna reddish brown with apical segments partly blackish; legs reddish brown ; length $3.5-4.0 \mathrm{~mm}$

Pronotum sparsely impressed by shallow punctures and their interstices nearly smooth, impunctate; bronzy black, violaceous blue or bluish green; antenna reddish brown with apical segments partly blackish; legs reddish brown; length $2.9-3.5 \mathrm{~mm}$.

langbianicus

11 (9). Elytral punctures about as large as interspaces or larger, and with some irregular transverse rugosities at side and with or without slightly raised longitudinal costae apically; pronotal punctures nearly as large as interspaces at side

Elytral punctures distinctly smaller than interspaces, and without irregular transverse rugosities or raised longitudinal costae ........................ 14

12 (11). Pronotum with lateral margin narrower and interstices of punctures finer and sparsely impressed; antenna reddish brown with apical segments partly blackish

Pronotum with lateral margin wider and interstices of punctures distinctly and rather closely impressed; antenna blackish with basal segments partly brownish; elytron with irregular transverse rugosities at side but interstices of semiregularly arranged longitudinal punctures at subapical portion hardly raised; green, cupreous green, violaceous blue or bluish black; legs entirely greenish or bluish; length $4.5-7.0 \mathrm{~mm} \cdots \cdots . .$. paviei

13 (12). Oblong oval : interstices of semiregularly arranged longitudinal puncture rows slightly raised; dorsal surfaces greenish cupreous, antenna yellow- 


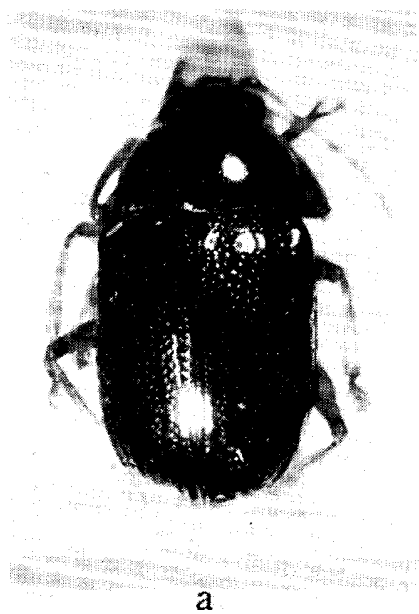

a

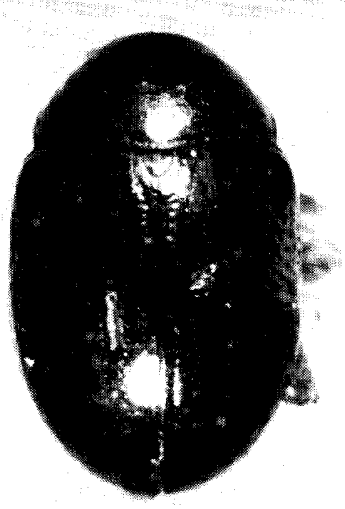

b

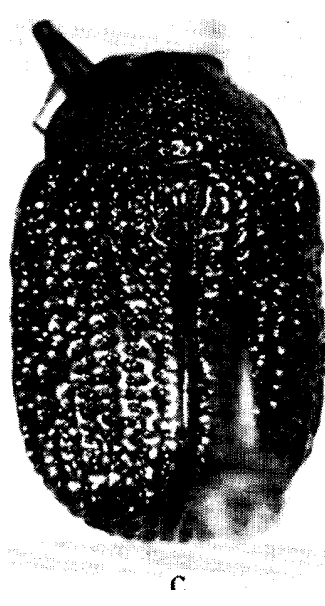

c.

Fig. 36. a, Colaspoides prasinus Lefèvre ; b, C. thailandicus Kimoto \& Gressitt, n. sp.; c, C. rugulosus Lefèvre.

ish brown with apical segments infuscate; length $6.3 \mathrm{~mm}$

fulvicornis

Short oval; interstices of punctures more distinctly raised; green with or without cupreous luster; legs reddish brown with femora greenish, or almost entirely greenish; antenna reddish brown with apical seg-

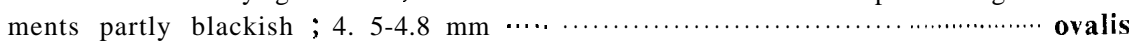

14 (11). Antenna normal, 3-7 segments of antenna not closely clothed with long hairs in male 15

Antenna with underside of 3-7 segments closely clothed with long hairs in male; coloration of dorsum variable, either reddish brown with metallic luster or aeneous; underside generally dark reddish brown, antenna reddish brown with apical segments infuscate ; length 4.3-G. 0 $\mathrm{mm}$

pilicornis

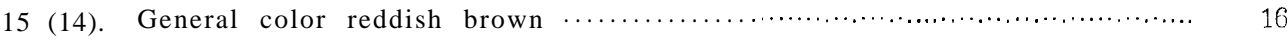

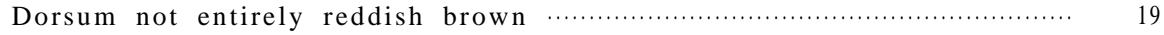

16 (15). Pronotal punctures distinctly smaller than interspaces even at side, or

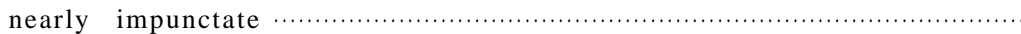

Pronotal punctures about as large as interspaces and slightly smaller at side ; reddish brown ; length $3.7-4.2 \mathrm{~mm}$............................. vietnamicus

17 (16). Pronotum distinctly but sparsely punctate; reddish brown, antenna reddish brown with apical segments blackish in various degrees, legs reddish brown with tibiae, tarsi and apex of femora dark brown to pitchy

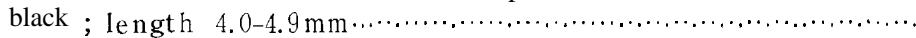

Pronotum nearly impunctate; pitchy brown with slight greenish luster ; antenna reddish brown with segment 11 mostly pitchy brown; abdomen and legs reddish brown; length 2.9-3.4 $\mathrm{mm} \ldots \ldots \ldots \ldots \ldots \ldots \ldots \ldots \ldots$ minimu

18 (15). Dorsal surfaces bluish, greenish or violaceous, ventral surfaces pitchy

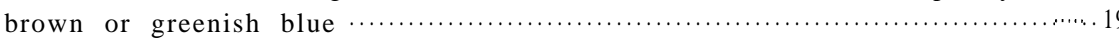

Pronotum reddish brown, elytron pitchy black; head and ventral surfaces reddish brown, antenna pitchy black with basal segments brownish ; legs reddish brown with tibiae, tarsi and apex of femora blackish ; pronotum distinctly and rather closely punctate, elytral punctures more closely and distinctly punctate than prothorax ; length 5. 1-5. $5 \mathrm{~mm}$ 
19 (15). Pronotum sparsely impressed by fine punctures and their interstices

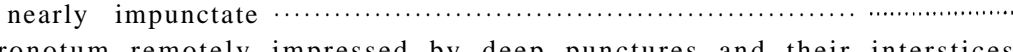

Pronotum remotely impressed by deep punctures and their interstices impressed by minute, round punctures; dorsal surfaces greenish with slight cupreous luster ; ventral surfaces pitchy brown; antenna reddish brown with apical segments infuscate, legs entirely reddish brown;

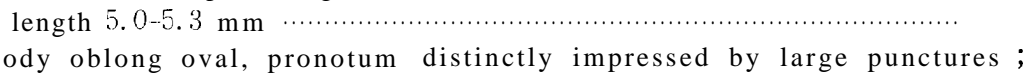
fulvipes

20 (19). Body oblong oval, pronotum distinctly impressed by large punctures; head and dorsal surfaces green with or without cupreous luster: antenna yellowish to reddish brown with apical segments infuscate; ventral surfaces pitchy brown, legs reddish brown; length $3.5-4.5 \mathrm{~mm} \cdots$ diffinis Body short oval, pronotum sparsely impressed with shallow punctures; head and dorsal surfaces green with or without cupreous luster; antenna reddish brown with apical segments pitchy black; ventral surfaces pitchy brown, legs reddish brown ; length 3. 5-3. $7 \mathrm{~mm}$

persimilis

Colaspoides chakratongii (Chûjồ) New combination (Fig. 40a)

Tailandiachakratongii Chûjô, 1964, Nature and Life in SE Asia, Kyoto 3: 283, fig. 18 (Thailand : Chieng Mai; CHUJO).

Distribution : Thailand.

Material examined. THAiland: Chiang Mai Prov., Doi Suthep, 1000 m, 1 ex., 12. vi. 1965, P. D. Ashlock (BISHOP), 1 ex., 8. vi. 1965, S. Asahina, 2 ex., 12. vi. 1965 , K. Morimoto, 16 exs., 18-19. vi. 1965, K. Morimoto \& Miyatake (KU).

\section{Colaspoides diffinis Lefèvre}

Colaspoides diffinis Lef., 1893, Ann. Soc. Entomol. Fr. 62: 134 (Tonkin). -Chen, 1935, Sinensia 6 (3) : 255 (S. China, Tonkin). Gressitt\& Kimoto, 1961, Pac. Insects Monogr. IA: 279 (Tonkin, S. China).

Distribution : S. China, Vietnam.

We could not trace the type location of this species. Our identification is based on the material identified by Lefèvre which is preserved in Paris Museum.

No additional material.

Colaspoides femoralis Lefèvre

Colaspoides femoralis Lef., 1885, Mém. Soc. Sci. Liège, ser. 2, 11: 158, nota 1 (China: Macao) ; 1889, Ann. Soc. Entomol. Fr. ser. 6, 9: 298 (Tonkin: Lien-Son) ; 1893, ibid. 1893: 133 (Cochinchine, Tonkin). - Chen, 1935, Sinensia 6 (3) : 258, fig. 10 (China, Macao, Tonkin, Cochinchina). -Gressitt \& Kimoto, 1961, Pac. Insects Monogr. IA: 280 (China).

Distribution: Laos, Vietnam, China.

Material examined. LAOS: Umgeb. Vanky, 1 ex., 1963; Umgeb. Paklay, 1 ex., 1963 (MUNCHEN). VIETNAM: $25 \mathrm{~km}$ SW of Pleiku, $400 \mathrm{~m}, 1$ ex., 12. v. 1960, L. W. Quate (BISHOP). 
Colaspoides fulvicornis (Baly)

Colasposoma fulvicorne Baly, 1865, Trans. Entomol. Soc. Lond., ser. 3, 2 (5) : 428 (Siam; BM). Colaspoides fulvicornis:Jacoby, 1889, Ann. Mus. Civ. Genova 27: 187, nota (Siam).

Distribution : Thailand.

No additional material.

\section{Colaspoides fulvipes Lefèvre}

Colaspoides f ulvipes Lef., 1889, Ann. Soc. Entomol. Fr. ser. 6, 9: 299 (Tonkin : Lien-Son).

Distribution : Vietnam.

No additional material.

Colaspoides langbianicus Kimoto \& Gressitt, n. sp. (Fig. 37a)

Body broadly ovate, strognly convex. Bronzy black, violaceous blue or bluish green, head with labrum reddish brown; antenna reddish brown with apical portion of eighth, ninth and most of eleventh segments blackish; ventral surfaces entirely reddish brown.

Head with surface finely granulate, sparsely impressed by fine punctures, vertex with a shallow longitudinal sulcus. Antenna short, with first segments large, robust, slightly curved; second robust, nealy as long as wide, shortest; third slenderer, nearly $12 / 3$ times as long as second; fourth slightly shorter than third; fifth subequal to fourth in length and shape; sixth sligthly shorter than fifth; seventh robuster, nearly $1 \mathrm{l} / \mathrm{Z}$ times as long as wide, and nearly $11 / 2$ times as long as sixth; eighth slightly shorter than seventh; ninth subequal to eighth in length but slightly slenderer ; tenth subequal to ninth in length

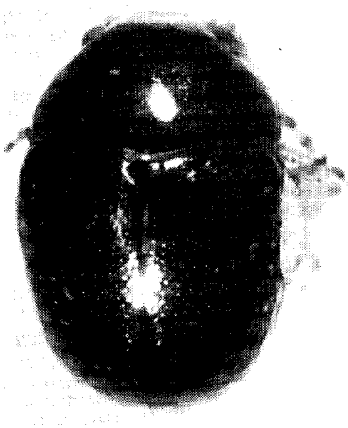

a

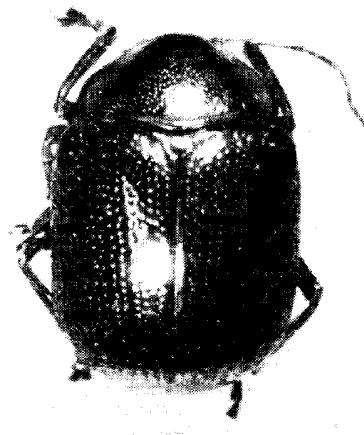

b

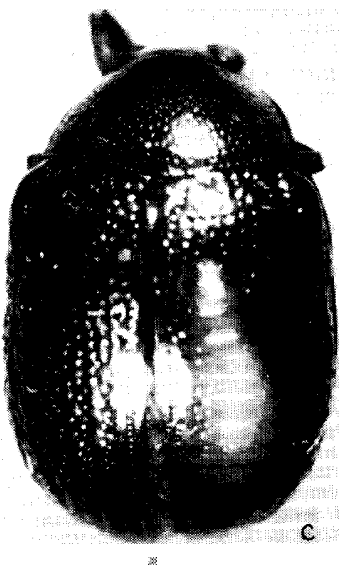

Fig. 37. a, Colaspoides langbianicus Kimoto \& Gressitt, n. sp.; b, C. paviei Lefèvre ; c, C. ovalis Lefèvre. 
and shape; eleventh sligthly longer than tenth and its apex pointed. Pronotum nearly twice as broad as long, strongly narrowed anteriorly, basal margin sinuate with median portion distinctly arched posteriorly, dorsal surface strongly convex, sparsely impressed by shallow punctures. Scutellum subpentagonal, surface somewhat wrinkled and impressed by fine punctures. Eiytron widest at humerus, slightly wider than pronotum, and strongly narrowed posteriorly, dorsal surface very strongly convex, distinctly and closely punctate, punctures partly arranged in longitudinal rows, their interstices closely impressed by fine punctures.

Length 2.9-3.5 mm.

Holotype (BISHOP), VIETNAM : Mt. Lang Bian, 1500-2000 m, 19. v.-8. vi. 1961, N. R. Spencer. Paratopotypes: 35 exs., same data as the holotype (BISHOP, KIMOTO). Paratypes : VIETNAM: Dilinh (Djiring), $1200 \mathrm{~m}, 22-28$. iv. 1960, L. W. Quate (BISHOP).

This new species clsoely resembles C. rufipes Kimoto \& Gressitt, but differs in having pronotum more sparsely impressed with shallow punctures and their interstices nearly impunctate.

Colaspoides laosensis Kimoto \& Gressitt, n. sp. (Fig. 38b)

Oblong oval; reddish brown, antenna with apical sements slightly darker, legs with tibiae, tarsi and apical part of femora infuscate in various degrees.

Head with surface smooth, sparsely impressed by distinct punctures, clypeus more closely punctate than vertex. Antenna slenderer, nearly $2 / 3$ as long as body length ; first segment large, robust, slightly curved; second robust, slightly longer than wide, shortest; third slenderer, nearly twice as long as second; fourth subequal to third in length and shape, fifth about $11 / 4$ times as long fourth; sixth slightly longer than fifth and much robuster apically; seventh subequal to sixth in length and shape; eighth slightly shorter than seventh; ninth and tenth subequal to eighth in length and shape; eleventh slightly longer than tenth and its apex pointed. Pronotum nearly $12 / 3$ times as broad as long, widest at $1 / 3$ from basal corner and strongly narrowed anteriorly and less strongly so posteriorly, basal margin sinuate with median portion distinctly arched posteriorly; dorsal surface convex, distinctly and rather closely punctate throughout and their interstices sparsely impressed by minute punctures. Scutellum subpentagonal, surface smooth, shining, impunctate. Elytron widest at humeral region, slightly wider than pronotum, and gradually narrowed posteriorly, dorsal surface strongly convex, distinctly and closely punctate, punctures partly arranged in longitudinal rows, and their interstices sparsely impressed by fine punctures.

Length 4.0-4.5 $\mathrm{mm}$.

Holotype (MUNCHEN), LAOS: Umgeb. Vanky, 1963. Paratopotypes : 4 exs., same data as the holotype (MUNCHEN, KIMOTO). Paratypes : LAOS: Umgeb. 
Paklay, 1 ex., 1963 (MUNCHEN) ; Ban Van Heue, 20 km E of Phou-kow-kuei, 2 exs., 1 15. v. 1965, J. A. Rondon (BISHOP).

This new species somewhat resembles C. vietnamicus Kimoto \& Gressitt, but differs in having the pronotal punctures finer and their interstices wider than their diameters.

Colaspoides martini Lefèvre

Colaspoides martini Lef., 1885, Bull. Entomol. Fr., ser. 6, 5: 66 (Tonkin;? PARIS). Chen, 1935, Sinensia 6 (3) : 252 (Sino-Tonkin frontier). Gressitt \& Kimoto, 1961, Pac. Insects Monogr. 1A: 282 (S. China, N. Vietnam).

Distribution : Thailand, Laos, Vietnam.

We could trace the type location of this species. Our identification is based on the material identified by Lefèvre. Those are preserved in Paris Museum.

Material examined. THAiland: Saraburi Prov., Ban Muak Lek Nat. Park, 1 ex., 5. vi. 1965, P. D. Ashlock; Nayok Prov., Kao Yai Nat. Park, 1 ex., 5. vi. 1965, P. D. Ashlock (BISHOP) ; Khao Yai Nat. Park, 26 exs., 5-6. vi. 1965, S. Asahina, K. Morimoto \& Y. Miyatake ; Fang, 1 ex., 13. vi. 1965, Y. Miyatake (KU) ; Saraburi, 49 exs., 21-23. vi. 1960 (BANGKHEN). LAOS: Ban Van Heue, $20 \mathrm{~km}$ E of Phou-kow-kuei, 9 exs., l-15. v. 1965, J. A. Rondon; Muong Sing, NW of Luang Prabang, $650 \mathrm{~m}, 2$ exs., 6-10. vi., 1960, L. W. Quate (BISHOP). VIETNAM: Hoa-Binh, Tonkin, 7 exs., A. Cooman (FREY).

Colaspoides minimus Kimoto \& Gressitt, n. sp. (Fig. 38c)

Oblong oval; generally reddish brown with or without bluish luster, especially on marginal portion.

Head with surface smooth, sparsely impressed by distinct punctures, which are more closely impressed on clypeus than vertex, vertex with a shallow longitudinal sulcus, clypeus not distinctly separated from vertex by transverse impression. Antenna slenderer, nearly $2 / 3$ as long as body length ; first segment large, robust, curved ; second robust, slightly longer than wide, shortest; third slenderer, almost $12 / 3$ times as long as second; fourth subequal to third in length and shape; fifth slightly longer than fourth; sixth subequal to fifth in length and shape; seventh slightly longer than sixth; eighth slightly shorter than seventh; ninth and tenth subequal to eighth in length and shape; eleventh slightly longer than tenth and its apex pointed. Pronotum nearly twice as broad as long, widest slightly before base, strongly narrowed anteriorly, basal margin sinuate with median portion slightly arched posteriorly, dorsal surface nearly impunctate. Scutellum subpentagonal, surface smooth, shining, impunctate. Elytron widest at humeral region, slightly wider than pronotum and strongly narrowed posteriorly, dorsal surface distinctly convex, distinctly and closely punctate and interstices of punctures subequal to or slightly narrower than their diameters in lateral portion, punc- 

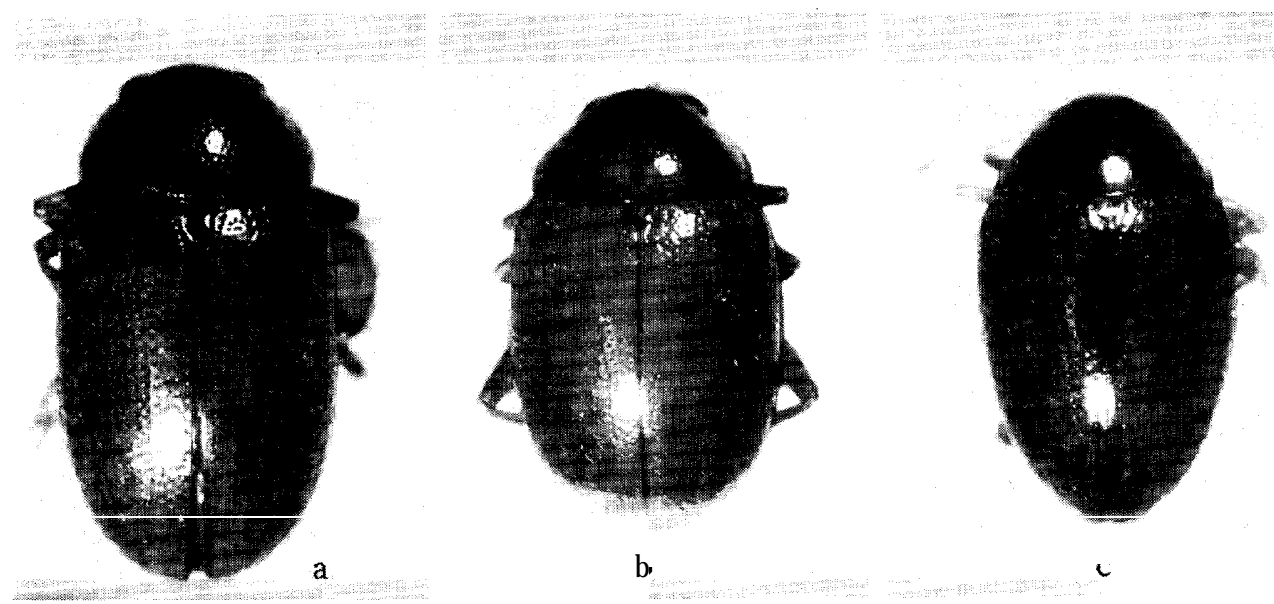

Fig. 38. a, Colaspoides vietnamicus Kimoto \& Gressitt, n. sp.; b, C. Iaosensis Kimoto \& Gressitt, n. sp.; C, C. minivnus Kimoto \& Gressitt, n. sp.

tures partly arranged in longitudinal rows.

Length $2.9-3.4 \mathrm{~mm}$.

Hozotype (BISHOP), VIETNAM : Karyu Danar, 200 m, 13-28. ii. 1961, N. R. Spencer. Paratopotypes : 3 exs., same data as the holotype (BISHOP, KIMOTO). Paratype : LAOS: Ban Van Eue, 20 km E of Phou-kow-kuei, 1 ex., 15-31. v. 1965, Native collr (BISHOP).

A specimen taken from Laos is slightly different from specimens from Vietnam, in having the body slightly longer and the dorsum entirely reddish brown without bluish luster.

This new species somewhat resembles C. laosensis Kimoto \& Gressitt, but differs in having pronotum nearly impunctate and the body length much short. er.

Colaspoides ovalis Lefèvre (Fig. $37 \mathrm{c}$ )

Colaspoides ovalis Lef., 1890, Nouv. Arch. Mus. Paris, ser. 3, 2: 201 (Laos: Luang-Phabang; PARIS) ; 1904, Miss. Pavie Indo-China, ser. 2, 3 (Zool.) : 156 (Laos),

Colaspoides siamensis Jacoby, 1905, Fasciculi Malayenses, Appendix 2 : 4 (Siamese Malay States: Biserat, Jalor; BM). New synonymy.

Distribution : Thailand, Laos, Vietnam.

Material examined. THAiland: Chiang Mai Prov., Fang (Agr. Exp. Station), 600 m, 1 ex., 14. vi. 1965, P. D. Ashlock (BISHOP) ; Fang, 4 exs., 14. vi. 1965, K. Morimoto \& Y. Miyatake ; Tankeo, Doi Suthep, 800 m, 8 exs., 10. vi. 1965, Y. Miyatake; Khao Yai Nat. Park, 4 exs., 6. vi. 1965, K. Morimoto \& Y. Miyatake; Khao Chong, nr. Trung, 7 exs., 25-27. vi. 1965, Y. Miyatake; Ratopoom, 2 exs., 27. vi. 1965, Y. Miyatake ; Kor Hong, 3 exs., 23. vi. 1965, K. Morimoto \& Miyatake ; Songkhla, 2 exs., 22. vi. 1965, Y. Miyatake (KU). LAOS: 
Ban Van Heue, $20 \mathrm{~km}$ E of Phou-kow-kuei, 5 exs., l-15. v. 1965, J. A. Rondon; Sayaboury Prov., Sayaboury, 2 exs., 5. v. 1966, Native collr (BISHOP). VIETNAM: $7 \mathrm{~km}$ SE of Dilinh (Djiring), $990 \mathrm{~m}, 1$ ex., 2. v. 1960, R. E. Leech ; Ban Me Thuot, $500 \mathrm{~m}, 1$ ex., 16-18. v. 1960, L. W. Quate; $20 \mathrm{~km} \mathrm{~N}$ of Pleiku, $650 \mathrm{~m}$, 1 ex., 9. v. 1960, L. W. Quate (BISHOP) ; Hoa-Binh, Tonkin, 1 ex. (FREY).

\section{Colaspoides paviei Lefèvre (Fig. 37b)}

Colaspoides paviei Lef., 1890, Nouv. Arch. Mus. Paris, ser. 3, 2: 201 (Laos: Luang-Prabang;

PARIS) ; 1904, Miss. Pavie Indo-China, ser. 2, 3 (Zool.): 156 (Laos).

Distribution : Laos.

Material examined. LAOS: Umgeb. Vientiane, 20 exs., iii-vi. 1963; Umgeb. Paklay, 4 exs., 1963 ; Umgeb. Pakse, 5 exs., 1963; Umgeb. Vanky, 4 exs., 1964 (MUNCHEN) ; Vientiane Prov., Ban Van Eue, 6 exs., 15. v. 1965, 1 ex., 31. v. 1966, 1 ex., 30. vi. 1966, Native collr; BanVan Heue, 20 km E of Phou-kowkuei, 2 exs., l-15. v. 1965, 6 exs., 15.-31. v. 1965, Native collr ; Attopeu Prov., Houei Kong, 1 ex., 16. v. 1965, 3 exs., 31. v. 1965, Native collr; Khamouane Prov., Phou Tiou, 1 ex., 17. v. 1965, Native collr (BISHOP).

Colaspoides persimilis Kimoto \& Gressitt, n. sp. (Fig. 39b)

Body short oval, strongly convex. Bluish green, with or without cupreous luster ; head with labrum reddish brown; antenna reddish brown with four or five apical segments blackish; ventral surfaces pitchy black; legs entirely reddish brown.

Head with surface smooth, sparsely impressed by fine punctures, vertex with a shallow longitudinal sulcus, clypeus not distinctly separated from vertex by transverse impression. Antenna slenderer, nearly $2 / 3$ as long as body length ; first segment large, robust, slightly curved; second robust, slightly longer than wide, shortest; third slenderer, nearly twice as long as second; fourth subequal to third in length and shape; fifth nearly $11 / 3$ times as long as fourth; sixth subequal to fifth in length and shape; seventh subequal to sixth in length but distinctly widened apically; seventh to tenth subequal to each other in length and shape; eleventh slightly longer than tenth and its apex pointed. Pronotum nearly twice as broad as long, strongly narrowed anteriorly, basal margin sinuate with median portion distinctly arched posteriorly, dorsal surface strongly convex, sparsely impressed by fine punctures. Scutellum subpentagonal, surface smooth, nearly impunctate. Elytron widest at humeral region, slightly wider than pronotum, and gradually narrowed posteriorly, dorsal surface strongly convex, distinctly and closely punctate, punctures partly arranged in longitudinal rows, and their interstices sparsely impressed by minute punctures. 
Length $3.5-3.7 \mathrm{~mm}$.

Holotype (BISHOP), LAOS: Ban Van Eue, $20 \mathrm{~km}$ E of Phou-kow-kuei, l-15. v. 1965, J. A. Rondon. Paratopotypes: 12 exs., same data as the holotype (BISHOP, KIMOTO). Paratypes : LAOS: 1 ex., same data as the holotype, but malaise trap ; Ile de Khong, 1 ex., 7. v. 1965, Native collr (BISHOP).

This new species most closely resembles C. diffinis Lefevre, but differs in having the body much robuster, punctures of pronotum more sparsely and finely impressed.

Colaspoides piceus Kimoto \& Gressitt, n. sp. (Fig. 39a)

Oblong oval; general color reddish brown, elytron pitchy black with margins more brownish; antenna pitchy black with basal segments brownish; legs reddish brown with tibiae, tarsi and apex of femora blackish.

Head with surface smooth, shining, sparsely impressed by distinct punctures which are more closely impressed on clypeus than vertex; clypeus not distinctly separated from vertex by transverse impression. Antenna relatively robsut, nearly $2 / 3$ as long as body length and slightly depressed at subapical segments; first segment large, robust, slightly curved ; second robust, slightly longer than wide, shortest; third slenderer, nearly twice as long as second; fourth subequal to third in length and shape; fifth $11 / 3$ times as long as fourth, slightly depressed ; sixth subequal to fifth in length and shape; seventh slightly longer than sixth; eighth slightly shorter than seventh; ninth and tenth subequal to eighth in length and shape; eleventh slightly longer than tenth and its apex pointed. Pronotum nearly $14 / 5$ times as broad as long, widest almost at middle, strongly narrowed anteriorly and less strongly so posteriorly, basal margin sinuate with median portion slightly arched posteriorly ; dorsal surface convex, distinctly and rather closely punctate. Scutellum subpentagonal, surface smooth, shining, impunctate. Elytron widest at humeral region, slightly wider than pronotum, and gradually narrowed posteriorly, surface strongly and more closely punctate than pronotum, punctures partly arranged in longitudinal rows, and their interstices sparsely impressed by minute punctures.

Length 5.1-5.5 mm.

Holotype (BISHOP), VIETNAM: Ban Me Thuot, 500 m, 16-18. v. 1960, L. W. Quate. Paratopotype : 1 ex., same data as the holotype (KIMOTO). Paratype : LAOS: Ban Van Heue, $20 \mathrm{~km}$ E of Phou-kow-kuei. 1 ex., 1-15. 1965, J. A. Rondon (BISHOP).

This new species somewhat resembles C. pilicornis Lefevre, but differs in having the punctures of pronotum stronger, and antenna without any long hairs in male. 


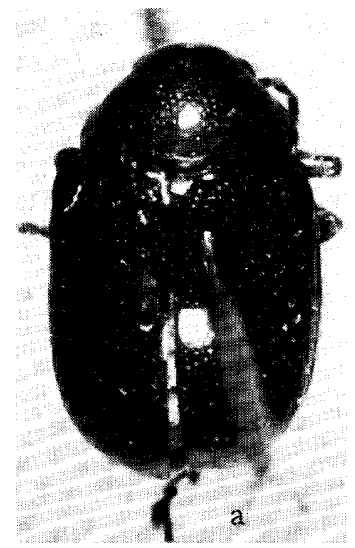

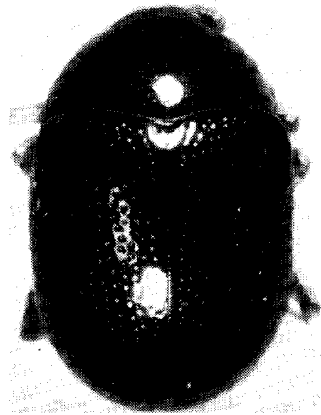

$\mathrm{b}$

Fig. 39. a, Colaspoides pice us Kimoto \& Gressitt, 11. sp. ; b, C. persimilis Kimoto \& Gressitt, n. sp.

\section{Colaspoides pilicornis Lefevre}

Colaspoides pilicornis Lef., 1889, Ann. Soc. Entomol. Fr. ser. 6, 9:298 (Tonkin: Lien-Son), -Chen, 1935, Sinensia 6 (3) :254 (S. China, Tonkin). -Gressitt \& Kimoto, 1961, Pac.

Insects Monogr. 1A:282 (Tonkin, S. China).

Distribution : Vietnam, S. China.

We could not trace the type location of this species. Our identification is based on the material identified by Lefevre, which is preserved in Paris $\mathrm{Mu}$ seum.

Material examined. VIETNAM: $7 \mathrm{~km}$ SE of Dilinh (Djiring), 2 exs., 2. v. 1960, R. E. Leech; Ban Me Thuot, 500 m, 2 exs., 16-18. v. 1960, S. Quate \& L. W. Quate (BISHOP) ; Hoa-Binh, Tonkin, 32 exs., A. Cooman (FREY).

Colaspoides prasinus Lefevre (Fig. 36a)

Colaspoides prasina Lef., 1890, Nouv. Arch. Mus. Paris, ser. 3, 2 : 202 (Laos: Luang-Prabang;

PARIS); 1904, Miss. Pavie Indo-China, ser. 2, 3 (2001.) : 156 (Laos).

Colaspoides chlorolaema Berlioz, 1917, Ann. Soc. Entomol. Fr. 86: 316 (Laos; PARIS). New synonymy.

Distribution: Thailand, Laos.

Material examined. THAildAND: Chiang Mai Prov., Maesa, 3 exs., 16. vi. 1965, P. D. Ashlock; Fang, 500 m, 1 ex., 13. vi. 1965, P. D. Ashlock (BISHOP) ; Mae Sa Water Fall, 10 exs., 16. vi. 1965, K. Morimoto \& Y. Miyatake; Doi Suthep, $1000 \mathrm{~m}, 2$ exs., 10. vi. 1965, K. Morimoto; Fang, 2 exs., 14. vi. 1965, K. Morimoto (KU). LAOS: Muong Sing, NW of Luang Prabang, 650 m, 2 exs., 610. vi. 1960, S. Quate, \& L. W. Quate; Vientiane, 1 ex., 31. v.-3. vi. 1960, S. Quate \& L. W. Quate (BISHOP). 
Colaspoides rufipes Kimoto \& Gressitt, n. sp. (Figs. 40b-c)

Body broadly ovate, strongly convex. Bronzy black; head cuprous with labrum reddish brown; antenna reddish brown with apical portion of eighth, ninth and tenth and entire eleventh segments blackish; ventral surface entirely black; legs entirely reddish brown.

Head with surface finely granulate, sparsely impressed by fine punctures, vertex with a shallow longitudinal sulcus, clypeus separated from vertex by feeble transverse impression. Antenna short, with first segment large, robust, slightly curved; second robust, slightly longer than wide, shortest; third slenderer, $11 / 3$ times as long as second; fourth subequal to third in length and shape ; fifth slightly shorter than fourth; sixth subequal to fifth in length and shape; seventh robuster, nearly $11 / 2$ times as long as wide, and nearly $11 / 2$ times as long as sixth; eighth subequal to seventh in length and shape; ninth subequal to eighth in length but slightly slenderer; tenth subqual to ninth in length and shape; eleventh slightly longer than tenth and its apex pointed. Pronotum nearly twice as broad as long, strongly narrowed anteriorly, basal margin sinuate with median portion distinctly arched posteriorly, dorsal surface strongly convex, distinctly and fairly closely punctate throughout and interstices of punctures closely impressed by minute punctures. Scutellum subpentagonal, surface finely granulate and impressed by distinct punctures. Elytron widest at humerus, slightly wider than pronotum, and strongly narrwoed posteriorly, dorsal surface very strongly convex, distinctly and closely punctate, punctures partly arranged in longitudinal rows, and their interstices closely impressed by fine punctures.

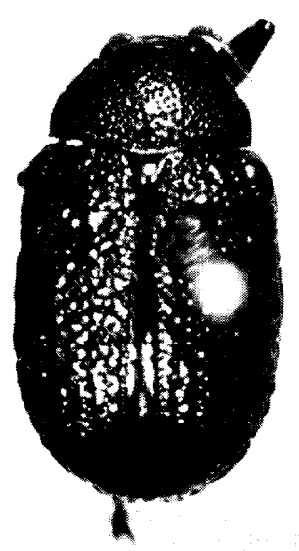

a

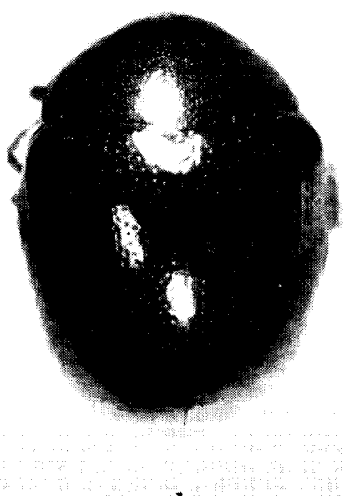

b

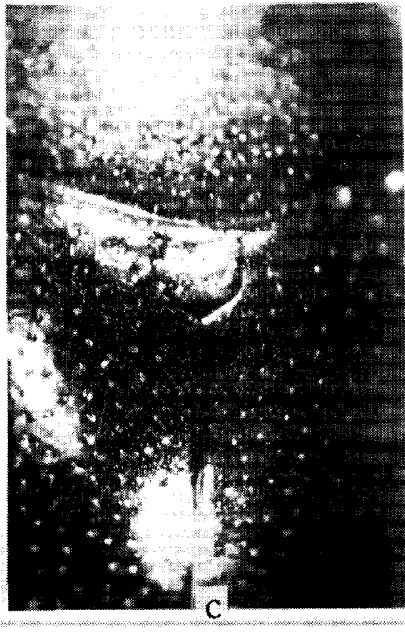

Fig. 40. a, Colaspoides chakratongii (C h ûjô) ; b, C. rufipes Kimoto \& Gressitt, n. sp., C, ditto, enlarged. 
Length $3.5-4.0 \mathrm{~mm}$.

Holotype (BISHOP), VIETNAM: $15 \mathrm{~km} \mathrm{NW}$ of Dalat, $1850 \mathrm{~m}, 5 . \mathrm{v} .1960$, S. Quate. Paratopotype : 1 ex., same data as the holotype (KIMOTO). Paratypes: VIETNAM: $15 \mathrm{~km}$ Dalat, $1500 \mathrm{~m}, 1$ ex., 29. iv.-5. v. 1960, S. Quate; $6 \mathrm{~km}$ S., Dalat, 1400-1500 m, 4 exs., 9. vi.-7. vii. 1961, N. R. Spencer (BISHOP).

Distribution : Vietnam.

This new species somewhat resembles $\mathrm{C}$. diffinis Lefevre, in having the dorsum greenish and legs entirely reddish brown, but differs in being the body length shorter and antenna robuster and head with vertex finely granulate.

\section{Colaspoides rugipennis Lefevre}

Colaspoides rugipennis Lef., 1893, Ann. Soc. Entomol. Fr. 62 : 133 (Indo-China ; PARIS). -Berlioz, 1917, Ann. Soc. Entomol. Fr. 86: 316 (Haut-Laos: Vientiane).

Distribution : Laos.

No additional material.

Colaspoides rugulosus Lefevre (Fig. 36c)

Colaspoides rugulosa Lef., 1889, Ann. Soc. Entomol. Fr. ser. 6, 9: 299 (Tonkin).

Distribution : Vietnam.

Material examined. VIETNAM: Dalat, $6 \mathrm{~km} \mathrm{S,} \mathrm{1400-1500} \mathrm{m,} 2$ exs., 9. vi.7. vii. 1961, N. R. Spencer (BISHOP).

\section{Colaspoides spinigerus Lefevre}

Colaspoides spinigera Lef., 1893, Ann. Soc. Entomol. Fr. 62: 133 (Saigon).

Distribution : Vietnam.

We could not trace the type location of this species. No additional material.

Colaspoides thailandicus Kimoto \& Gressitt, n. sp. (Fig. 36b)

Body broadly ovate, strongly convex. Greenish blue with or without cupreous luster, head with labrum reddish brown; antenna reddish brown with apical portion of tenth and most of eleventh segments blackish; ventral surfaces entirely pitchy black; legs entirely reddish brown.

Head with surface smooth, distinctly and not closely punctate, vertex with a shallow longitudinal sulcus, clypeus separated from vertex by feeble transverse impression. Antenna slenderer, nearly $2 / 3$ as long as body length; first segment large, robust, slightly curved ; second robust, slightly longer than wide, shortest; third slenderer, nearly twice as long as second; fourth subequal to third in length and shape; fifth slightly longer than fourth; fifth to seventh subequal to each other in length and shape; eighth slightly shorter 
and robuster than seventh; eighth to tenth subequal to each other in length and shape; eleventh slightly longer than tenth and its apex pointed. Pronoturn nearly $12 / 3$ times as broad as long, strongly narrowed anteriorly, basal margin sinuate with median portion distinctly arched posteriorly, dorsal surface strongly covex, sparsely impressed by fine punctures. Scutellum subpentagonal, surface smooth, shining, impunctate. Elytron widest at humerus, slightly wider than pronotum, and gradually narrowed posteriorly, dorsal surface very strongly convex, distinctly and closely punctate, punctures partly arranged in longitudinal row, and their interstices sparsely impressed by minute punctures. Posterior femur with distinct spine and anterior femur widened, angulate at middle.

Length 3.5-4.6 $\mathrm{mm}$.

Hодотүре (Type No. 2361, Kyushu Univ.), THAILAND: Mae Sa Water Fall, Chiang Mai Prov., 16. vi. 1965, Y. Miyatake. Paratopotypes: 5 exs., same data as the holotype (KU, BISHOP, KIMOTO). Paratypes : THAILAND : Botanical Garden, Chiang Mai, 2 exs., 12. vi. 1965, Y. Miyatake ; Chiangmai, 1 ex., 9. vi. 1965, K. Morimoto; Fang, 1 ex., 14. vi. 1965, K. Morimoto; Chieng Dao, 1 ex., 15. vi. 1965, Y. Miyatake (KU) ; Mae Sa, Chiang Mai Prov., 2 exs., 16. vi. 1965, P. D. Ashlcok; Chiang Mai Prov., Chiangmai (Arboretum), 2 exs., 12. vi. 1965, P. D. Ashlock (BISHOP).

This new species resembles C. martini Lefèvre, but differs in having pronotum more strongly produced posteriorly at middle, posterior femur with a spine shorter and the ventral surfaces of body pitchy black.

Colaspoides vietnamicus Kimoto \& Gressitt, n. sp. (Fig. 38a)

Oblong oval; entirely reddish brown.

Head with surface smooth, shining, rather closely impressed by distinct punctures, clypeus not distinctly separated from vertex by transverse impression. Antenna relatively robust, nearly $2 / 3$ as long as body length; first segment large, robust, slightly curved; second robust, almost as long as wide, shortest; third elongate, nearly three times as long as wide, and nearly twice as long as second; fourth subequal to third in length and shape ; fifth slightly longer than fourth; sixth subequal to fifth in length and shape; seventh slight ly longer than sixth; eighth slightly shorter than seventh; ninth and tenth subequal to eighth in length and shape; eleventh slightly longer than tenth and its apex pointed. Pronotum nearly $12 / 3$ times as broad as long, widest almost at middle and strongly narrowed anteriorly and less strongly so posteriorly, basal margin sinuate with median portion distinctly arched posteriorly, and lateral margin rather widely marginate, dorsal surface convex, distinctly and rather closely punctate throughout, interstices of punctures finely impressed by minute punctures. Scutellum subpentagonal, surface smooth, shining, nearly impunctate. Elytron widest at humerus, slightly wider than pro. 
notum, and strongly narrowed posteriorly, dorsal surface very strongly convex, distinctly and closely punctate, punctures partly arranged in longitudinal rows, and their interstices sparsely impressed by minute punctures.

Length $3.7-4.2 \mathrm{~mm}$.

Holotype (BISHOP), VIETNAM: Karyu Danar, 200 m, 13-28. ii. 1961, N. R. Spencer. Paratopotypes: 2 exs., same data as the holotype (BISHOP, KIMOTO).

This new species somewhat resembles C. laosensis Kimoto \& Gressitt, but differs in having the pronotal punctures stronger and closer, and their interstices nearly as wide as their diameters or narrower.

\section{Genus Platycorynus Chevrolat}

Platycorynus Chevr., 1937, in Dejean, Cat. Coleopt. ed. 3: 413 (type: Eumolpus compressicornis Fabricius, from W. Africa). --Monrós\& Bechyné, 1956, Entomol. Arb. 7: 1127. -Gressitt \& Kimoto, 1961, Pac. Insects Monogr. 1A: 286. -Kimoto, 1964, J. Fac Agr. Kyushu Univ. 13 (2): 241.

Corynodes Hope, 1840, Coleopt. Manual. 3: 162. -Marshall, 1864, Proc. Linn. Soc. Lond., Zool. 8: 30. --Baly, 1867, Trans. Entomol. Soc. Lond., ser. 2, 4: 99. -Chapuis, 1874, Genera Coleopt. 10: 337-Lefèvre. 1885, Mém. Soc. Sci. Liège, ser. 2, 11: 146. Jacoby, 1908, Fauna India, Coleopt. 2: 492 (type designated as Eumolpus compressicornis Fabricius). Chen, 1934, Sinensia 5(5-5):484; 1935, ibid. 6(3):265; 1940, ibid. 11 (5 6): 422 . -Chû jô, 1956, Philip. J. Sci. 85(1): 171 .

\section{KeY to SPECIES OF Platycorynus}

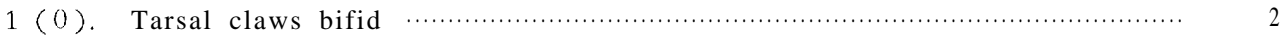

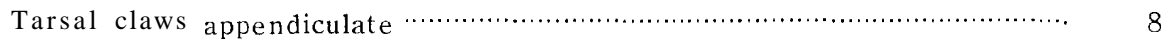

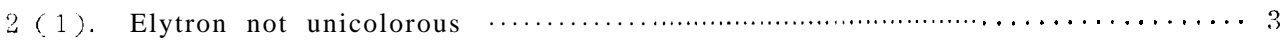

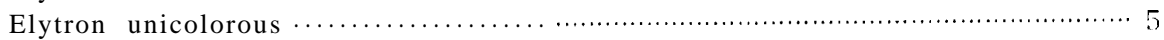

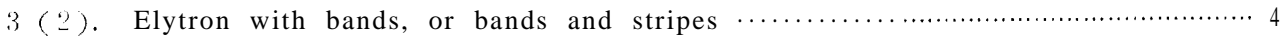

Elytron cupreous, a humeral spot and apex purplish or violaceous;

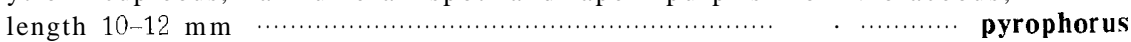

4 (3). Dark to greenish blue; elytron reddish violacoeus with antemedian arcuate marking and its apex dark blue; sulcus behind eye wider and fovea between eyes deeper ; length $10-11 \mathrm{~mm}$. .................... arcuatefasciatus

Blue, greenish or violet; elytron violaceous with basal and postmedian band reddish violaceous, more or less golden reddish to greenish at base and behind middle and anterior to middle and at apex; sulcus behind eye narrower and fovea between eyes shallower; length 9-

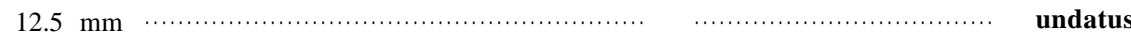

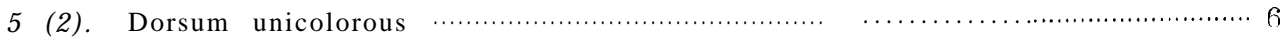

Pronotum metallic blue violet; elytron cupreous red with violaceous

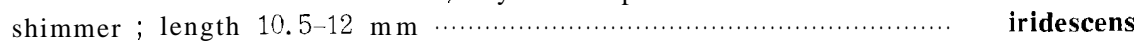

6 (5). Punctures of elytron finer, interspaces of punctures much wider than

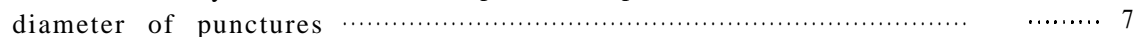

Punctures of elytron stronger. interstices of punctures narrower or subequal to diameter of punctures, especially on lateral and laterosubapical portion; front of head with a pair of depressions or transverse furrow between eyes; but the furrow does not connect with ocular groove ; length 9-12 mm

peregrinus 
7 (6). Excavation of interocular space deep and strong; ocular groove with wide and deep; entirely bluish black; length 10-12 mm

Excavation of interocular space weak, indistinct; ocular groove narrow

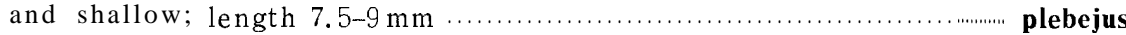

8 (2). Front of head with a complete transverse furrow between eyes $\ldots . . . \ldots \ldots \ldots \ldots . . . .6$

Front of head lacking a complete transverse furrow between eyes $\ldots \ldots \ldots \ldots \ldots . \ldots$

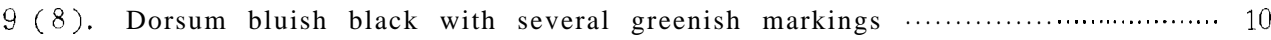

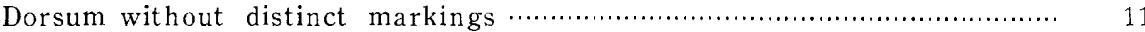

10 (9). Dorsum bluish black, elytron with basal and postmedian markings bluish to reddish green; vertex strongly convex ; length $7.7-8.0 \mathrm{~mm}$..... igneofasciatus

Dorsum bluish black; pronotoum with median and lateral markings reddish to bluish green, elytron with basal, postmedian and apical markings reddish to bluish green; vertex convex; length 6.9-8.3 $\mathrm{mm} \cdots$ pyrospilotus

$11(10)$. Vertex not strongly raised above level of interocular area $\ldots \ldots \ldots \ldots \ldots \ldots \ldots \ldots . . . \ldots 12$

Vertex strongly raised above level of interocular area; apical 5 antennal segments wider than long; length $9 \mathrm{~mm} \ldots \ldots \ldots \ldots \ldots \ldots \ldots \ldots \ldots \ldots \ldots$ gratiosus

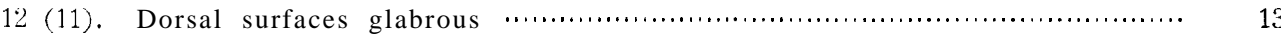

Dorsal surfaces entirely covered with tine hairs, elytron closely and rugosely punctate; golden green ; length $10.5 \mathrm{~mm} \ldots \ldots \ldots \ldots \ldots \ldots \ldots \ldots$ rugipennis

13 (12). Preapical antennal semgents nearly 1 1/3 times as long as wide $\ldots \ldots \ldots \ldots \ldots \ldots \ldots \ldots$

Preapical antennal segments nearly as long as wide; greenish, violaceous or blackish blue, in some specimen elytron greenish cupreous with pronotum violaceous blue; length $7-11 \mathrm{~mm} \ldots \ldots \ldots \ldots \ldots \ldots \ldots \ldots$ chalybaeus

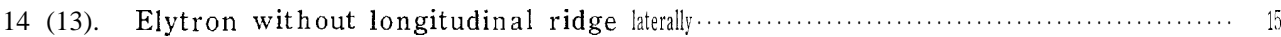

Elytron with strongly raised 2 longitudinal ridges laterally in female and punctures somewhat in paired rows, with spaces between pairs in part slightly raised; last 5 apical segments slightly longer than broad; generally metallic blue to violaceous; length $5.8-8$. $5 \mathrm{~mm}$

aemulus

15 (14). Elytron without an acute costa posterolaterally ; in male mid femur with a spine and mid tibia with an excavation at middle; golden green to bluish green; length $9.5-11 \mathrm{~mm}$

Elytron with an acute costa at side from before middle to apex in female; middle femur slightly dilated into a small tooth beneath; bluish green; length $8.3-10 \mathrm{~mm}$

nitidus

16 (8). Elytron with a sharply raised lateral costa starting from humerus and

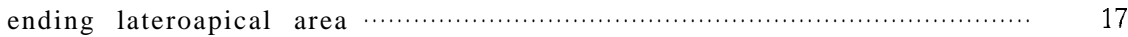

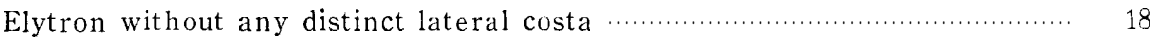

17 (16). Elytron sparsely covered with fine hairs at apical portion ; dorsum distinctly punctate and their interstices smooth and shining; golden

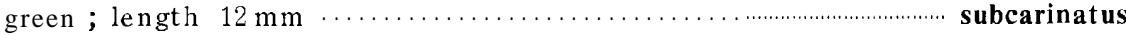

Elytron glabrous; dorsum rugosely impressed by large punctures and their interstices raised; golden green; length $6.9-8.0 \mathrm{~mm}$................. rugosus

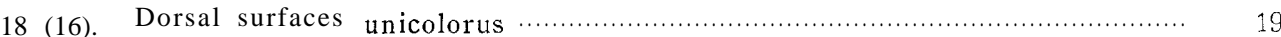

Dorsal surfaces more than one color...................................... 20

19 (18). Elytron covered with fine hairs subapically; head without distinct interantennal cavity; preapical antennal segments distinctly longer than

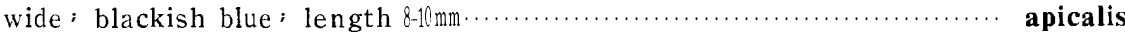

Elytron entirely glabrous; head with interocular cavities moderately deep, somewhat oblique or with side sloping upward to margin above antennal insertion; prepaical antennal segments about as broad as long; blackish blue with slight purplish or greenish reflections; length 
9-12. $5 \mathrm{~mm}$

mouhoti

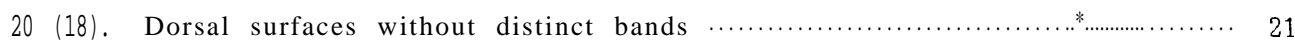

Pronotum bluish black, elytron with basal, median and apical bands

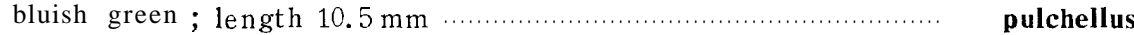

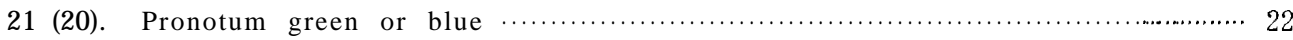

Pronotum generally reddish cupreous ; elytron largely golden green, changing to violet along suture broadly and external margin narrowly;

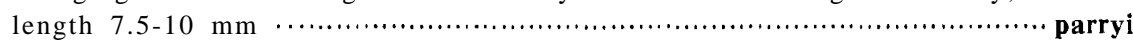

22 (21). Elytral punctures very coarse and irregular; elytron reddish cupreous with external marign green ; length $12.5 \mathrm{~mm}$........................ chapanus

Elytron densely and strongly punctures ; punctures subgeminately arranged, especially near suture; elytron reddish cupreous with external margin greenish cupreous; length $8 \mathrm{~mm}$....

speciosus

\section{Platycorynus aemulus (Lefèvre)}

Corynodes aemulus Lef., 1889, Ann. Soc. Entomol. Fr., 6, 9: 297 (Tonkin: Lien-Son ; PARIS) ; 1893, ibid. 1893: 130 (Tonkin, Lang-son). -Chen, 1934, Sinensia 5 (5-6) : 503; 1935, ibid. 6 (3) : 270 (N. Vietnam).

Corynodes beauchenei Lef., 1889, Ann. Soc. Entomol. Fr., ser. 6, 9: 298 (Tonkin: Lien-Son; PARIS) ; 1893, ibid. 1893: 130 (Tonkin, Lang-Son), -Chen, 1934, Sinensia 5(5-6): 520 (Chinese border, Tonkin); 1935, ibid. 6 (3) : 270 (Chinese border, Tonkin). New synonymy.

Corynodes costatus var. inlimbatus Pic, 1936, Mél. Exot. Entomol. 67: 13 (Tonkin).

Platycorynus aemulus: Gressitt \& Kimoto, 1961, Pac. Insects Monogr. 1A: 289 (Tonkin).

Platycorynus beauchenei : Gressitt \& Kimoto, 1961, Pac. Insects Monogr. 1A : 289 (N. Vietnam, Chinese border).

This species is characteristic in having the elytron with sharply raised two longitudinal ridges laterally in female. Pic (1936) described custata var. inlimbatus from Tonkin, separating the nominate form in having elytron entirely bluish, instead of bluish with margin violaceous. The nominate form of custatus was described by Baly (1864) from Manila. Chûjô (1964) recorded this species from Thailand, but this should be referred under Platycorynuschalybaeus (Marshall).

DistRIBUTION : Laos, Vietnam.

Material eXamined. LAOS: Umgeb. Paklay, 1 ex., 1963 (MUNCHEN) ; Vientiane Prov., Ban Van Eue, 1 ex., 15. v. 1965. Native collr (BISHOP). VIETNAM: Hoa-Binh, Tonkin, 2 exs., A. Cooman (FREY).

Platycorynus apicalis Kimoto \& Gressitt, n. sp. (Fig. 41a)

Oblong ; entirely blackish blue.

Head with transverse furrow separating anterior margin of vertex from clypeus widely interrupted at middle; surface subrugosely impressed by large punctures and interspaces of pucntures much narrower than their diameters, finely granualte and thickly covered by fine hairs. Antenna slightly over half as long as body length and spical four segments strongly depressed; first seg- 


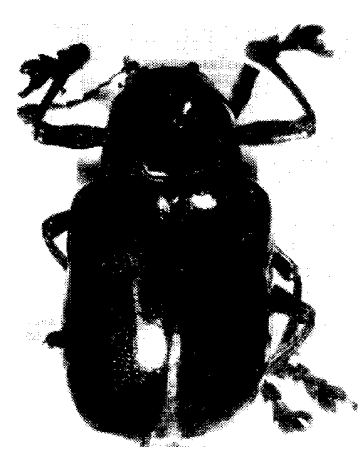

a

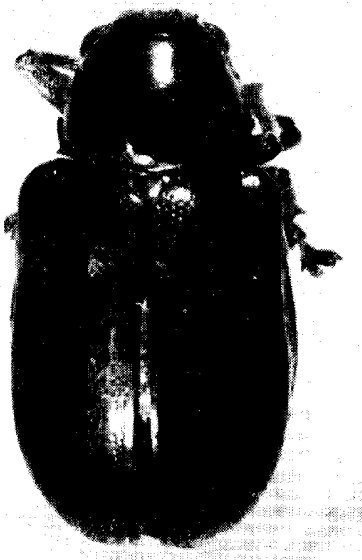

$\mathrm{b}$

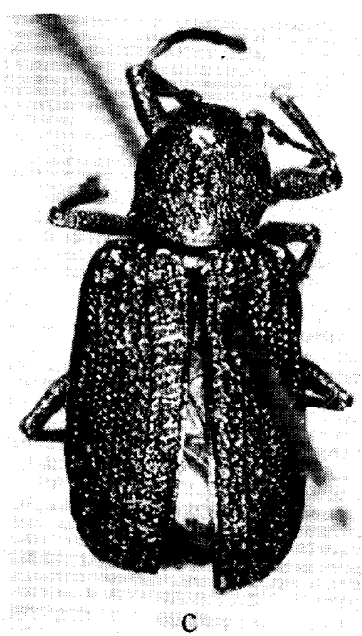

Fig. 41. a, Platycorynus apicalis Kimoto \& Gressitt, n. sp. ; b, P. laosensis Kimoto \& Gressitt, n. s p.; c, P. rugosus Kimoto \& Gressitt, n. sp.

ment robust, subglobose, nearly $12 / 3$ times as long as wide ; third $11 / 2$ times as long as second, slender; fourth slightly longer than third; fifth slightly longer than fourth; sixth nearly $4 / 5$ as long as fifth and widened apically; seventh nearly $1 \frac{1 / 2}{2}$ times as long as sixth, and neariy $11 / 2$ times as long as wide, subtriangular; eighth nearly $4 / 5$ as long as seventh and slightly longer than wide; ninth and tenth subequal to eighth in length and shape; eleventh nearly $11 / 4$ times as long as tenth and its apex pointed. Pronotum convex, $12 / 5$ times as wide as long; side rounded, widest almost at middle, distinctly narrowed anteriorly and posteriorly ; surface distinctly and not closely impressed by large and small punctures, and interstices of punctures smooth, shining, and closely covered by fine hairs at lateroanterior part. Scutellum subtriangular, slightly longer than broad, rounded apically, surface smooth, shining, nearly impunctate. Elytron distinctly convex subbasally, and separated from behind by a deep transverse furrow; dorsum distinctly and rather closely punctate, and covered with fine hairs subapically. Tarsal claws appendiculate.

Length $8.0-10 \mathrm{~mm}$.

ноцотуре (MUNCHEN), LAOS : Umgeb. Vientiane, iii-vi. 1963. Paratypes : 2 exs., same data as the holotype (BISHOP, KIMOTO).

This new species somewhat resembles P.mouhoti Baly, but differs in having the lateroanterior part of pronotum and apical part of elytron thickly covered by fine hairs.

\section{Platycorynus arcuatefasciatus (Pic) New combination (Fig. 42e)}

Corynodes arcuatefasciatus Pic, 1938, Mél. Exot. Entomol. 70: 27 (Tonkin ; PARIS). 
Distribution : Vietnam.

No additional material.

Platycorynus chalybaeus (Marshall) (Fig. 43b)

Corynodes chalybaeus Marshall, 1865, J. Linn. Soc. 2001. 8: 44 (Cambodia, Burma ; BM). -Jacoby, 1889, Ann. Mus. Civ. Genova 27: 182 (Burma). -Lefèvre, 1890, Nouv. Arch.

Mus. Paris, ser. 3, 2: 200 (Laos) ; 1904, Miss. PavieIndo-China, ser. 2, 3 (Zool.): 155 (Laos).

Corynodes pavieiLefèvre, 1890, Nouv. Arch. Mus. Paris, ser. 3, 2: 198 (Cambodge ; PARIS) ; 1904, Miss. PavieIndo-China, ser. 2, 3 (Zool.): 154 (Cambodge). New synonymy.

Corynodes dilaticollis Jacoby, 1892, Ann. Mus. Civ. Genova 32: 916 (Burma ; GENOVA, BM) ; 1908, Fauna India, Coleopt. 2: 52 (Burma). N ew synonymy.

Corynodes rectesulcatusPic, 1938, Bull. Soc. Entomol. Fr. 43: 124 (Yunnan ; PARIS). -Gressitt \& Kimoto, 1961, Pac, Insects Monogr. 1A: 290 (=chalybaeus).

Corynodes chrysochoides Ch e n, 1940, Sinensia 11(5-6): 523 (Hainan). -Gressitt \& Kimoto, 1961, Pac. Insects Monogr. 1A : 290 (=chalybaeus).

Platycorynus chalybaeus: Gressitt \& Kimoto, 1961, Pac. Insects Monogr. 1A : 290 (China, Vietnam, Thailand) .

Platycorynus dilaticollis: Chûjô, 1964, Nature and Life in SE Asia, Kyoto 3: 276 (Cambodia). Platycorynus beauchenei: Chûjô, 1964, ibid. (Thailand).

Distribution: Burma, Thailand, Cambodia, Laos, Vietnam, China.

Material examined. THAILAND : Doi Suthep, $1000 \mathrm{~m}$, Chinag Mai Prov., 1 ex., 12. vi 1965, Y. Miyatake, 1 ex., 19. vi. 1965, K. Morimoto (KU) ; Kanchanaburi, 2 exs., 21. v. 1962 (BANGKHEN). LAOS: Umgeb. Vientiane, 224 exs., i-vi. 1963, Umgeb. Pakley, 34 exs., 1963; Umgeb. Vanky, 5 exs., 1963, 12 exs., 1964 (MUNCHEN) ; Vientiane Prov., Ban Van Eue, 14 exs., 15. v. 1965, 1 ex., 31. vii. 1965, 1 ex., 16. iii. 1966, Native collr; Vientiane Prov., Phou Kou Khouei, 1 ex., 30. iv. 1966, Native collr ; Ban Van Heue, $20 \mathrm{~km}$ E of Phou Kou Khuei, 1 ex., 1-15. v. 1965, J. A. Rondon, 1 ex., 15-31. v. 1965, Native collr ; Borikhane Prov., Pakkading, 4 exs., 17. v. 1965, Native collr ; Sedone Prov., Pakse, 1 ex., 16. v. 1965, Native collr ; Sayaboury Prov., Sayaboury, 1 ex., 5. v. 1966, 1 ex., 20. v. 1966, Native collr; Moung Sing, NW of Luang Prabang, $650 \mathrm{~m}, 1$ ex., 6-10. 1960, S. Qaute \& L. W. Quate ; Luang Prabang, 300 m, 1 ex., 4-5. vi. 1960, S. Quate (BISHOP). VIETNAM: Ban Me Thuot, 500 m, 1 ex., 16-18. v. 1960, S. Quate ; $7 \mathrm{~km} \mathrm{SE}$ of Dilinh (Djiring), 990 m, 1 ex., 2 v. 1960, R. E Leech ; $20 \mathrm{~km} \mathrm{~N}$ of Pleiku. $650 \mathrm{~m}, 1$ ex., 9. v. 1960, L. W. Quate (BISHOP).

\section{Platycorynus chapanus (Chen)}

Corynodes chapanus Chen, 1934, Sinensia 5(5-6) : 498, fig. 25 (Chapa,Tonkin; PARIS) ; 1935, ibid. 6 (3) : 269 (Tonkin).

Platycorynus chapanus: Gressitt \& Kimoto, 1961, Pac. Insects Monogr, 1A: 290 (Tonkin).

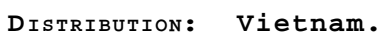


Platycorynus deletus (Lefèvre)

Corynodes deletus Lef ., 1890, Nouv. Arch. Mus. Paris, ser. 3, 2: 119 (Laos ; PARIS) ; 1904, Miss. Pavie Indo-China, ser. 2, 3 (Zool.) : 115 (Laos).

Corynodes fonkineus Lefèvre, 1893, Ann. Soc. Entomol. Fr. 62: 132 (Tonkin: Lang-Son; cotype :MCZ). New synonymy.

Platycorynus viridimicans Gressitt \& Kimoto, 1961, Pac. Insects Monogr. 1A: 295 (S. China, Hainan I. ; BISHOP), New synonymy.

Distribution: S. China, Hainan, Vietnam, Laos.

No additional material.

\section{Platycorynus florentini (Lefèvre) New combination}

Corynodes florentini Lef., 1893, Ann. Soc.Entomol. Fr. 62: 130 (Tonkin ; Lang-Son).

Distribution : Vietnam.

We could not trace the type location of this species. Judging from the original description, this species seems to be very close to or same as a variation of P.chalybaeus Marshall. This species is not included in the key,

\section{Platycorynus gratiosus Baly}

Platycorynus graitosus Baly, 1864, Descr. n. gen. \&spec. Phytoph., 7 (Triganee; BM). Gressitt \& Kimoto, 1961, Pac. Insects Monogr. 1A: 291 (Hainan, Laos, Vietnam).

Corynodes gratiosus: Marshall, 1865, J. Linn. Soc. 2001. 8: 41 (Rangoon). -Baly, 1867, Trans. Entomol. Soc. Lond., ser. 3, 4(2): 127 (Malaya: Tringanee; Burma, Cambodia). -- Jacoby, 1889, Ann. Mus. Civ. Genova, 27: 181 (Burma) ; 1908, Fauna India, Coleopt. 2: 498 (Burma, Malay Pen., Cambodia). Chûjô, 1961, Nauture and Life in SE Asia, Kyoto 1: 349, fig. 3 (Thailand: Chieng Mai).

Corynodes gibbifrons Lefèvre, 1885, Mém. Soc. Sci. Liège, ser. 2, 11: 148, nota 2 (Siam: Juthia) ; 1890, Nouv. Arch. Mus. Paris. ser. 3, 2: 199 (Cambodge) ; 1893, Ann. Soc. Entomol. 1893: 130 (Tonkin); 1904, Miss. Pavie Indo-China, ser. 2, 3 (2001.) : 154 (Cambodge). New synonymy.

Distribution : Burma, Thailand, Cambodia, Laos, Vietnam, Hainan.

We could not trace the type location of Corynodes gibbifrons Lefèvre. In Paris Museum, a specimen identified by Lefèvre as gibbifrons is preserved. This specimen was taken from Cambodia and seems to be a series of Pavie Expedition.

Also, this species closely resembles C. chalybaeus (Marshall), but differs in having the subbasal depression of elytron weaker, lateraoapical portion of elytron not angulate and punctures of pronotum much stronger.

Material exAmined. THAILAND : Kanchanaburi, 1 ex., 21. v. 1962 (BANGKHEN). LAOS: Borikhane Prov., Pakkading, 5 exs., 17. v. 1965, 2 exs., 29. iv. 1966, 1 ex., 13. v 1966, Native collr; Khammouane Prov., Phon Tiou, 1 ex., 17. v. 1965, 1 ex., 11. ix. 1965, Native collr; Ban Heue, $20 \mathrm{~km} \mathrm{E}$ of Phoukow-kuei, 1 ex., 15-31. v. 1965, Native collr (BISHOP) ; Umgeb. Vientiane, 10 exs., iii- vi. 1963, Umge b. Paklay, 4 exs., 1963; Umgeb. Pakse, 2 exs., 1963; Umgeb. Vanky, 2 exs., 1963 (MUNCHEN). 


\section{Platycorynus igneofasciatus (Baly) New combination (Fig. 42b)}

Corynodes igneofasczatus Baly, 1860, J. Entomol. 1: 32 (Cochinchina, Siam; BM) ; 1893, Ann.

Soc. Entomol. 1893 : 130 (Cochinchina).

Corynodes pyrosphilotus varr. inapicalis, perroudi Pic, 1934, Mél. Exot. Entomol. 64: 4 (Cochinchina ; PARIS).

Corynodes satoi Chûjô, 1959, Sci. Report Agr. Expedition Cambodia, Hyogo Univ. Agr., 2: 58, 1 fig. (Cambodia; CHUJO). New synonymy.

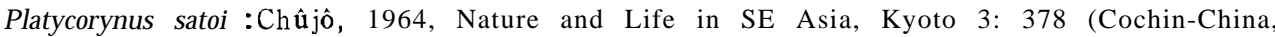
Thailand).

Distribution: Thailand, Cambodia, Laos, Vietnam.

Material examined. LAOS : Sedone Prov., Pakes, 4 exs., 17 v. 1965, Native collr ; Ile de Khong, 1 ex., 7. v. 1965, 2 exs., 17. v. 1965, Native collr (BISHOP). VIETNAM: Cochinchina, 2 exs. (FREY).

\section{Platycorynus iridescens (Berlioz) New combination}

Corynodes iridescens Belrioz, 1917, Ann. Soc. Entomol. Fr., 86: 314 (Laos; PARIS).

Distribution: Laos.

No additional material.

Platycorynus Zaosensis Kimoto \& Gressitt, n. sp. (Fig. 41b)

Oblong ; entirely bluish black.

Head with a pair of deep subtriangular depression between upper lobe of eyes; ocular groove wide and deep, sparsely covered by fine hairs; vertex moderately swollen, distinctly and not very closely punctate; clypeus more closely and distinctly punctate than vertex and interspaces of punctures slightly narrower than their diameters. Antenna sligthly over half as long as body length and strongly depressed except for same basal segments; first segment robust, subglobose, nearly $11 / 3$ times as long as wide; second subglobose, nearly as long as wide; third nearly $11 / 2$ times as long as second, slender; fourth subequal to third in length and shape ; fifth nearly $12 / 3$ times as long as fourth, and slightly widened apically ; sixth subequal to fifth in length but slightly wider ; seventh nearly 1 1/5 times as long as sixth, and as long as wide, subtriangular; eighth subequal to seventh in length and shape; ninth slightly longer than eighth and slightly wider than long; tenth subequal to ninth in length and shape; eleventh slightly longer than tenth, and nearly $11 / 5$ times as long as wide, and apex pointed. Pronotum convex, slightly broader than long; sides subparallel at basal $2 / 3$ and gradually narrowed anteriorly; surface sparsley impressed by distinct punctures, interstices of punctures sparsely impressed by minute puncutres. Scutellum subtriangular, slightly longer than broad, rounded apically, surface smooth, shining, impunctate, slightly depressed basally. Elytron distinctly convex subbasally, and separated from behind by a distinct transverse furrow; dorsum distinctly and not so 
closely punctate and their interstices nearly smooth, sparsely impressed by minute punctures. Claws bifid.

Length 10-12 mm.

Holotype (MUNCHEN), Laos : Umgeb. Vientiane, iii-vi. 1963. Paratypes : 6 exs., same data as the holotype (MUNCHEN, BISHOP, KIMOTO).

This new species somewhat resembles P.plebejus (Weise), but differs in having the excavation of interocular space deep and strong and ocular groove wide and deep.

\section{Platycorynus mouhoti Baly}

Platycorynus mouhoti Baly, 1864, Descr. n. gen. \&spec. Phytoph., 7 (Cambodia ; B M). -Gressitt \& Kimoto, 1961, Pac. Insects Monogr. 1A: 293 (key). -Kimoto, 1972, Entomol. Ts. 93 (4) : 145 (Annam).

Corynodes mouhoti: Jacoby, 1889, Ann. Mus. Civ. Genova 27: 180 (Burma). -Lefèvre, 1889, Ann. Soc. Entomol. Fr., ser. 6, 9: 294 (Cochinchine: Saigon) ; 1890, Nouv. Arch. Mus. Paris, ser. 3, 2: 198 (Cambodge, Siam) ; 1904, Miss. Pavie Indo-China, ser. 2, 3 (Zool.): 153 (Cambodge, Siam). -Jacoby, 1908, Fauna India, Coleopt. 2: 496 (Sikkim, Burma, Siam, Cambodia).

Corynodes bicavifrons Chen, 1940, Sinensia 11(5-6): 524 (Hainan). New synonymy.

Platycorynus bicavifrons: Gressitt \& Kimoto, 1961, Pac. Insects Monogr. 1A: 289 (Hainan, S. China).

Distribution : N. India, Burma, Thailand, Cambodia, Laos, Vietnam, Hainan, S. China.

Material EXAmined. THAILAND: Mae Klang Water Fall, nr. Chom Thong, 9 exs., 11. vi. 1965, K. Morimoto \& Y. Miyatake; Doi Suthep, 1000 m, Chieng Mai Prov., 1 ex., 12. vi. 1965, K. Morimoto; Khao Yai Nat. Park, '2 exs., 5. vi. 1965, S. Asahina \& K. Morimoto; Kor Hong, 1 ex., 23. vi. 1965, K. Morimoto (KU) ; Musa, nr. Fang, 1 ex., 22. xi. 1968, K. Hatta (EHIME) ; Kanchanaburi, 1 ex., 21. v. 1962 (BANGKHEN). LAOS: Umgeb. Vientiane, 61 exs., iii-vi. 1963 ; Umgeb. Pakse, 5 exs., 1963, 3 exs., 1964; Umgeb. Vanky, 6 exs., 1963; Umgeb. Paklay, 13 exs., 1963 (MUNCHEN) ; Vientiane Prov., Phou Kou Kouei, 1 ex., 31. v. 1965, Native collr ; Vientiane Prov., Ban Van Eue, 3 exs., 15. v. 1965, 1 ex., 31. vii. 1965, 5 exs., 15. vii. 1966, Native collr, 3 exs., 1-15. v. 1965, J. A. Rondon; Khammouane Prov., Phon Tiou, 3 exs., 17. v. 1965, 2 exs., 28. vi. 1965, 1 ex., 11. ix. 1965, Native collr ; Borikhane Prov., Pakkading, 2 exs., 17, v. 1965, 3 exs., 29. iv. 1966, 1 ex., 13. v. 1966, Native collr ; Wapikhamthong Prov., Khong Sedone, 1 ex., 16. v. 1965, Native collr; Tonpheng, 1 ex., 30. vi. 1966, Native collr (BISHOP). VIETNAM: Ban Me Thuot, 500 m, 4 exs., 16-18. v. 1960, R. E. Leech; Nam Fyan, 900-1000 m, 1 ex., 11. vii. -9. viii. 1961, N. R. Spencer; Than Moi, Tonkin, 3 exs., vi-vii. H. Fruhstorfer; Hoa-Binh, Tonkin, 5 exs., A. Cooman (FREY). 

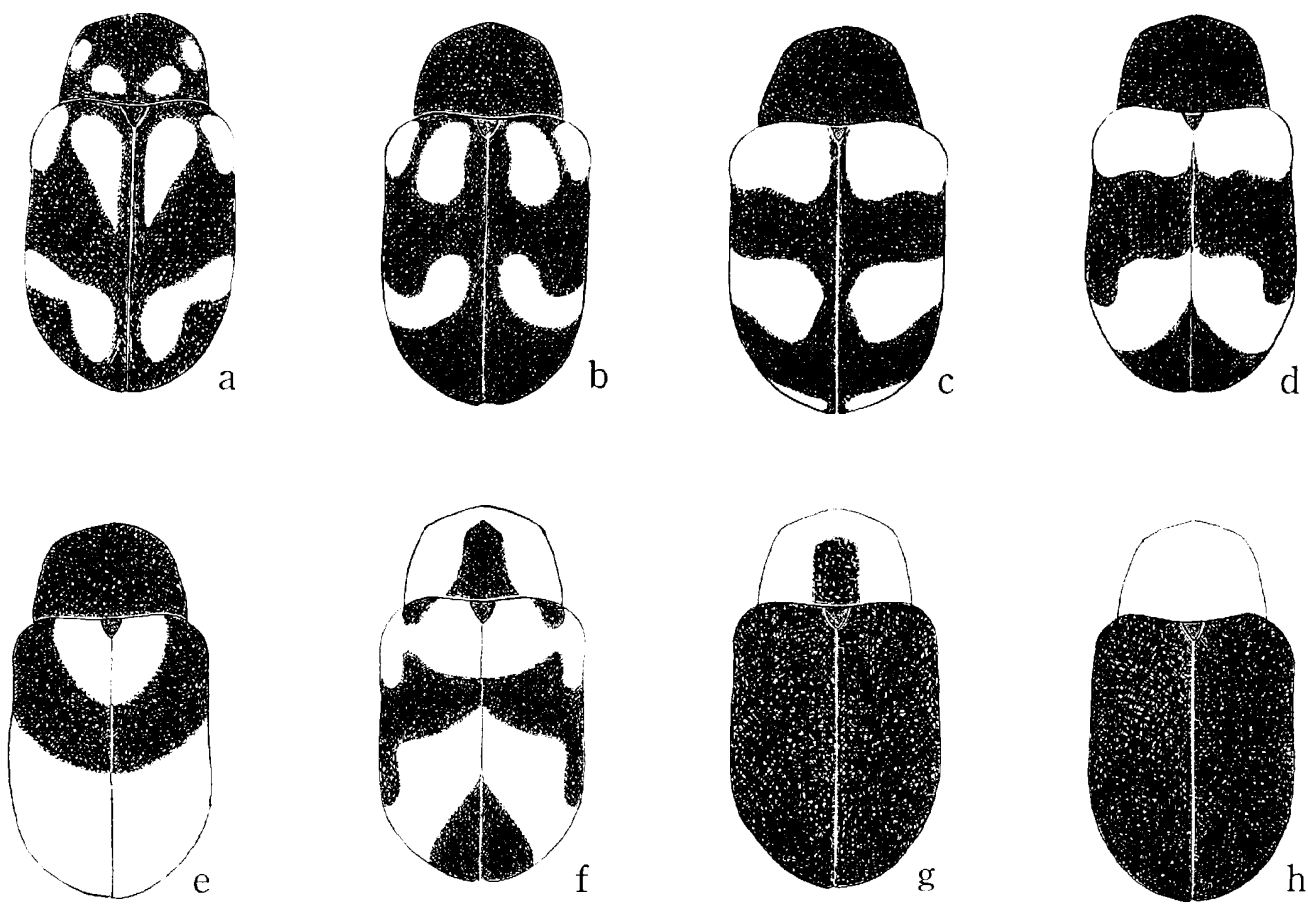

Fig. 42. a, Platycorynus pyrophorus (Parry) ; b, P. igneofasciatus (B aly) ; c, P. pulchellus (Baly) ; d, P. undatus (Olivier) ; e, P. arcuatejasciatus (Pic); f-h, Chrysochus pulcher Baly.

\section{Platycorynus nitidus (Fabricius)}

Chrysomela nitidus F., 1792, Entomol. Syst. 1: 325 (Siam).

Platycorynus approximans Baly, 1864, Descr. n. gen. \& spec. Phytoph., 6 (Sumatra). Jacoby, 1908, Fauna India, Coleopt. 2: 506 (=nitidus).

Corynodes nitidus: Marshall, 1865, J. Linn. Soc.Zool., 8: 37 (S. India). Jacoby, 1908, Fauna India, Coleopt. 2: 506 (India, Penang, Suamtra, Java).

Corynodes hyacinthinus Marshall, 1865, J. Linn. Soc. Zool. 8: 42 (Java). -Jacoby, 1908, Fauna India, Coleopt. 2: 506 (=nitidus).

Corynodes approximans: Baly, 1867, Trans. Entomol. Soc. Lond., ser. 3, 4(2): 107 (Sumatra, Pulo Penang, Malacca).

Corynodes amoenus Lefèvre, 1891, C. R. Soc. Entomol. Belg. 35: 277 (Upper Tenasserim). -Jacoby, 1908, Fauna India, Coleopt. 2: 506 (= nitidus).

Distribution : India, Burma, Thailand, Malaya, Sumatra, Java.

Material examined. Thailand: Khao Chong, nr. Trung, 5 exs., 25-26. vi. 1965, Y. Miyatake (KU).

\section{Platycorynus parryi Baly}

Platycorynus parryi Baly, 1864, J. Entomol. 2 : 223 (China; BM). -Gressitt \& Kimoto, 1961, Pac. Insects Monogr. 1 A: 293 (China, Korea).

Corynodes parryi:Lefèvre, 1893, Ann. Soc. Entomol. Fr. 1893: 130 (Tonkin, Lang-Son, HaLang); 1934, Sinersia 5 (5-6): 496 (China, Tonkin: Lang-Son, Cao-Bang); 1935, ibid. 
6(3): 226 (China, Tonkin).

Corynodes parryi var. jeanvoinei Pic, 1936, Mel. Exot. Entomol. 67: 13 (Tonkin).

Distribution: Korea, China, Vietnam.

No additional material.

\section{elatycorynus peregrinus (Herbst)}

Cryptocephalus peregrinus Herbst, 1783, in Fiiessly, Archiv d. Insectengesch. 5: 63, pl. 23, fig. 25 (Ostindien).

Eumolpuscyaneus Oliviter, 1808, Entomologie 6: 388, pl. 1, fig. 4, a, b (nec Fabricius, 1801). ---Marshall, 1865, J. Linn. Soc. Zool. 8: 34 (=peregrinus).

Corynodes peregrinus: Marshall, 1865, J. Linn. Soc. Zool. 8: 34 (India). -Baly, 1867, Trans. Entomol. Soc. Lond., ser. 3, 4(2): 132 (Malacca: Tringanee ; Siam, India). -Jacoby, 1889, Ann. Mus. Civ. Genova 27: 184 (Burma). -Lefèvre, 1889, Ann. Soc. Entomol. Fr., ser. 6, 9: 296 (Tonkin: Lien-Son) ; 1890, Nouv. Arch. Mus. Paris, ser. 3, 2: 198; 1904, Miss. PavieIndo-China, ser. 2, 3 (Zool.) : 154 (Laos, Battambang, Chataboun). -Jacoby, 1908, Fauna India, Coleopt. 2: 493, fig. 168 (India, Ceylon, Burma, Siam, Malacca). --Chen, 1934, Sinensia 5 (5-6) : 490; 1935, ibid. 6 (3) : 267 (S. China, Tonkin, Laos, Burma, Siam, E. India, Ceylon, Malacca).

Corynodes chapuisi Lefèvre, 1885, Mém.Soc. Sci. Liege, ser. 2, 11: 147, nota 1 (Pulo Penang). Jacoby, 1908, Fauna India, Coleopt. 2: 493 (=peregrinus).

Corynodes bonneuili Pic, 1934, Mél. Exot, Entomol. 64: 6 (Siam; PARIS).

Platycorynus major Gressitt \& Kimoto, 1961, Pac. Insects Monogr. 1 A : 292 (S. China, Tonkin ; MCZ). N ew synonymy.

Platycorynus peregrinus: Gressitt \& Kimoto, 1961, Pac. Insects Monogr. 1A: 294 (SW China, Laos, Tonkin). -Kimoto \& Takizawa. 1973, Kontyû, Tokyo 41 (2) : 176 (Nepal).

Distribution : India, Ceylon, Burma, Nepal, Thailand, Cambodia, Laos, Vietnam, SW China, Malaya.

Gressitt \& Kimoto (1961) described P.major as an indendent species. However, this is nothing but an infraspecific variation of this species.

Material ExAmined. THAilAnd: Chiang Dao, Chiang Mai Prov., 1 ex., 15. vi. 1965, P. D. Ashlock, 1 ex., 5-11. iv. 1958, T. C. Maa; Saraburi Prov., Ban Muak Lek Nat. Park, 2 exs., 5. vi. 1965, P. D. Ashlock (BISHOP) ; Khon Kaen, 4 exs., 21. vii. 1972, M. Sato, 7 exs., x-xii. M. Sato (EHIME) ; Mae Klang Water Fall, nr. Chom Thong, 4 exs., 11. vi. 1965, K. Morimoto \& Y. Miyatake; Fang, 1 ex., 14. vi. 1965, Y. Miyatkae; Prabuddhabat, nr. Mae Trang, 1 ex., 7. vii. 1970, on citrus, K. Yano (KU) ; Kanchanaburi, 9 exs., 21. v. 1962 (BANGKHEN). LAOS: Umgeb. Vientiane, 20 exs., iii-vi. 1963; Umgeb. Paklay, 22 exs., 1963, 2 exs., 1964; Umgeb. Pakse, 6 exs., 1963 ; Umgeb. Vanky, 1 ex., 1963, 7 exs., 1964 (MUNCHEN) ; Sayaboury Prov., Sayaboury, 1 ex., 17. v. 1965, Native collr (BISHOP). VIETNAM: Nha Ho, 14km N, Phan Rang, 2 exs., 15. xi. 1960, C. M. Yoshimoto (BISHOP).

\section{Platycorynus plebejus (Weise) (Fig. 43a)}

Corynodes plebejus Weise, 1889, Horae Soc. Entomol. Ross. 23: 593 (W. China; ZMB). -Chen, 1934, Sinensia 5(5-6): 491; 1935, ibid. 6(3): 267 (W. China). 
Chrysochus yunnanus Pic, 1927, Mé1. Exot. Entomol. 48: 25 (Yunnan ; PARIS). -Chen, 1934, Sinensia 6(3): 274 (Yunnan). -Gressitt \& Kimoto, 1961, Pac. Insects Monogr. 1A: 294 (= plebejus).

Chrysochus gunnanus (sic) var. minutus Pic, 1929, Bull. Soc. Linn. Lyon 8 : 35 (China).

Corynodes yunnanus Pic, 1943, Mél. Exot. Entomol. 64: 5 (Yunnan ; PARIS). -Gressitt \& Kimoto, 1961, Pac. Insects Monogr. 1A: 294 (=plebejus).

Platycorynus plebejus: Gressitt \& Kimoto, 1961, Pac. Insects Monogr. 1A : 294 (W. China, Laos).

Distribution : W. China, Laos.

No additional material.

\section{Platycorynus pulchellus (Baly) New combination (Fig. 42c)}

Corynodes pulchellus Baly 1860, J. Entomol. 1: 31 (Siam; BM).

DisTRIBUTION : Thailand.

No additional material.

\section{Platycorynus pyrophorus (Parryj (Fig. 42a)}

Eumolpus pyrophorus Parry, 1843, Proc. Entomol. Soc. Lond., 1843: 87 (Assam; BM) ; 1844, Ann. Mag. Nat Hist. 14: 454 (Assam) ; 1845, Trans. Entomol. Soc. Lond., 1845: 86 (Assam).

Corynodes gloriosus Baly, 1859, Ann. Mag. Nat. Hist. ser. 3, 4: 125 (N. India ; BM). ---Marshall, 1865, J. Linn. Soc.Zool. 8: 34 (=pyrophorus).

Corynodes pyrophorus: Marshall, 1865, J. Linn. Soc. 2001. 8: 34 (Assam). -Jacoby, 1908, Fauna India, Coleopt. 2: 492 (Himalaya, Nepal, India, Burma, China). - Chen, 1934, Sinensia 5 (5-6) : 492, fig. 19; 1935, ibid. 6 (3) : 268 (China, Nepal, Tonkin, Burma, Assam, Himalaya).

Platycorynus pyrophorus: Gressitt \& Kimoto, 1961, Pac. Insects Monogr. 1A: 294 (key). - Kimoto \& Takizawa, 1973, Kontyû, Tokyo 41(2): 176 (Nepal).

Distribution : India, Nepal, Burma, Laos, Vietnam, China. Material exAmined. LAOS: Umgeb. Pakse, 1 ex., 1963 (MUNCHEN).

\section{Platycorynus pyrospilotus (Baly) New combination}

Corynodes pyrospilotus Baly, 1860, J. Entomol. 1: 32 (Tenasserim; BM). - Marshall, 1865, J. Linn. Soc.Zool. 8: 37 (Siam). --Lefèvre, 1889, Ann. Soc. Entomol. Fr. ser. 6, 9: 294 (Cambodge : Pnomh-Penh) ; 1890, Nouv. Arch. Mus. Paris, ser. 3, 2: 198 (Cambodge : Battambang à Pnomh-Penh) ; 1893, Ann. Soc. Entomol. Fr. 1893: 130 (Tonkin); 1904, Miss. PavieIndo-China, ser. 2, 3 (Zool.): 153 (Cambodge). -Jacoby, 1908, Fauna India, Coleopt. 2: 495, pl. 2, fig. 13 (Burma, Siam).

Distribution : Burma, Thailand, Cambodia, Laos.

Material EXAMINed. THAILAND: Kanchanaburi, 3 exs., 21. v. 1962 (BANGKHEN). LAOS: Ban Van Heue, 20 km E. of Phou-kow-kuei, 1 ex., 1-15. v. 1965, J. A. Rondon, 1 ex., 15-31. v. 1965, Native collr (BISHOP) ; Umgeb. Vientiane, 8 exs., iii-vi. 1963 ; Umgeb. Pakse, 1 ex., 1963; Umgeb. Paklay, 2 exs., 1963 ; Umgeb. Vanky, 1 ex., 1964 (MUNCHEN) ; "Laos”, 5 exs., (FREY). 
Platycorynus rugipennis (Jacoby) New combination

Corynodes rugipennis Jac., 1895, Stett. Entomol. Zeit. 56: 66 (Siam ; RM).

Distribution : Thailand.

No additional material.

Platycorynus rugosus Kimoto \& Gressitt, n. sp. (Fig. 41c)

Oblong; dorsal surfaces golden green, ventral surfaces with slight cupre. ous luster.

Head with transverse furrow separating anterior margin of vertex from clypeus interrupted at middle, sparsely covered with fine hairs, vertex moderately swollen and slightly depressed at anterior portion; surface subrugosely impressed by large punctures and interspaces of punctures much narrower than their diameters and impressed by minute pucntures. Antenna sligthly over half as long as body length, and five or six apical segments depressed; first segment robust, club-shaped, nearly $11 / 2$ times as long as wide; second subglobose, nearly half as long as first; third nearly twice as long as second, slender; fourth slightly shorter than third; fifth slightly longer than fourth; sixth nearly as long as fifth, slightly widened apically; seventh nearly $11 / 4$ times as long as sixth, subtriangular; eighth nearly $4 / 5$ as long as seventh and nearly twice as long as wide; ninth and tenth subequal to eighth in length and shape ; eleventh $11 / 4$ times as long as tenth and its apex pointed. Pronotum convex, slightly broader than long; side rounded, widest slightly before middle, distinctly narrowed anteriorly and more strongly so posteriorly; surface rugosely impressed by large punctures, interstices of punctures much narrower than their diameters and impressed by minute punctures. Scutellum subtriangular, slightly longer than broad, rounded apically, surface granulate and sparsely impressed by minute punctures. Elytron slightly convex subbasally, and with a sharply raised lateral costa starting from humerus and ending lateroapical area and several short costae medianly, dorsum rugosely impressed by large punctures and their interstices distinctly raised. Tarsal claws appendiculate.

Length 6.9-8.0 $\mathrm{mm}$.

Holotype (BISHOP), VIETNAM: Dilinh (Djiring), $920 \mathrm{~m}, 22-28$. iv. 1960, L. W. Quate. Paratopotype : 1 ex., same data as the holotype but $1200 \mathrm{~m}$ (KIMOTO).

This new species somewhat resembles P. subcarinatus (Pic), in having the lateral costa of elytron, but differs in being the body length shorter and having the elytron subrugosely punctate.

Platycorynus speciosus (Lefevre) New combination

Corynodes speciosus Lef., 1891, C. R. Soc. Entomol. Belg. 35: 276 (Sikkim; cotype ?: BM) ; 1893, Ann. Soc. Entomol. Fr. 1893: 130 (Tonkin: Ha-Lang). -Jacoby, 1908, Fauna India, Coleopt. 2 : 497 (Sikkim). -Kimoto\& Takizawa, 1973, Kontyûu, Tokyo 41(2): 176 (Nepal). 
Distribution : N. India, Nepal, Vietnam.

No additional material.

Platycorynus subcarinatus (Yic) New combination

Chrysochus subcarinatus Pic, 1927, Mél. Exot. Entomol. 48: 25 (Tonkin;PARIS).

Distribution: Vietnam.

No additional material.

Platycorynus undatus (Olivier) (Fig. 42d)

Cryptocephalus undatus Oliv., 1791, Encycl. Méth., 6: 614 (Indes orientales).

Eumolpus bifasciatus Olivier, 1808, Entomologie 6: 900, pl. 1, fig. 5 (no locality name cited). Baly, 1867, Trans. Entomol. Soc. Lond., ser. 3, 4(2):13 (- undatus).

Eumolpus groendalii Swartz, 1808, in Schbnherr, Synonymia Insectorum 1(2): 235, nota 1

(China). -Baly, 1867, Trans. Entomol. Soc. Lond., ser. 3, 4(2):13 (=bifasciatus).

Corynodes bifasciatus: Marshall, 1865, J. Linn. Soc. 2001. 8: 34 (China), -Baly, 1867, Trans.

Entomol. Soc.Lond., ser. 3, 4(2): 130 (Malacca, Siam, China, Pulo-Penang).

Corynodes flosculus Marshall, 1865, J. Linn. Soc.Zool. 8: 37 (Cambodia ; BM). Baly, 1867,

Trans. Entomol. Soc. Lond., ser. 3, 4(2):13 (=bifasciatus).

Corynodes undatus: Lefèvre, 1889, Ann. Soc. Entomol. Fr., ser. 6, 9: 294 (Cambodia: Pnomh-

Penh ; Cochinchina: Mytho) ; 1890, Nouv. Arch. Mus. Paris, ser. 3, 2: 197 (Cambodge,

Siam) ; 1904, Miss. PavieIndo-China, ser. 2, 3 (Zool.): 153 (Cambodia, Siam). -Jacoby,

1908, Fauna India, Coleopt. 2: 493, pl. 2, fig. 12 (India, Burma, Siam, China, Malacca,

Penang). - Chen, 1934, Sinensia 5 (5-6) : 493; 1935, ibid. 6 (3) : 268 (Hainan, Hong

Kong, Indo-China, Burma, Siam, India, Malacca, Pulo-Penang). -Chûjô, 1956, Philip.

J. Sci. 85(1): 174 (Formosa).
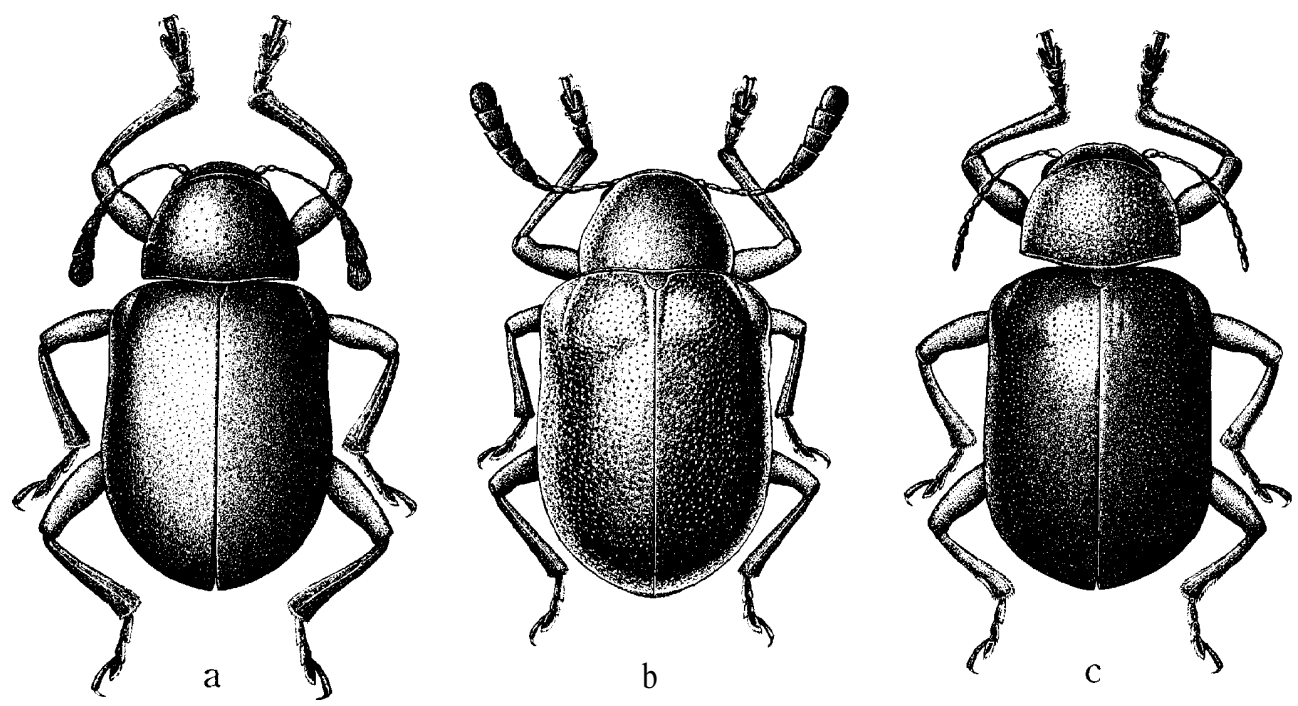

Fig. 43. a, Platycorynus plebejus Weise; b, P. chalybaeus (Marshall) ; c, Chrysochus pulcher Baly. 
Corynodes undatus varr. subconcolor, cyaneofasciatus Pic, 1934, Mél. Exot. Entomol. 64: 4 (Cambodia, Cochinchina).

Platycorynus undatus : Gressitt \& Kimoto, 1961, Pac. Insects Monogr. 1A: 259 (China, Hai$\mathrm{nan})$.

Distribution : India, Burma, Thailand, Cambodia, Laos, Vietnam, Hainan, China, Taiwan, Malaya.

MATERIAL EXAMINED. THAILAND: Bangkok, 1 ex., 22. vii. 1958 (BANGKHEN). LAOS: Wapikhamthong Prov., Khong Sedone, 1 ex., 16. v. 1965, 5 exs., 17. vii. 1965, Native collr; Khammouane Prov., Phon Tiou, 3 exs., 17. v. 1965, Native collr; Vientiane Prov., Phouei, 1 ex., 30. vi. 1966, Native collr ; Ban Van Heue, $20 \mathrm{~km}$ E of Phou Kow Kuei, 1 ex., l-15. v. 1965, J. A. Rondon (BISHOP) ; Umgeb. Vientiane, 60 exs., iii-vi. 1963; Umgeb. Pakse, 20 exs., 1963, 8 ex., 1964; Umgeb. Paklay, 14 exs., 1963 ; Umgeb. Vanky, 1 ex., 1964 (MUNCHEN). VIETNAM: Nha Ho, 14 km N, Phan Rang, 1 ex., 15. xi. 1960, C. M. Yoshimoto (BISHOP).

\section{Genus Chrysochus Chevrolat}

Chrysochus Chevrolat, 1837, in Dejean, Cat. Coleopt. ed. 3: 413. -Chapuis, 1874, Genera Coleopt. 10: 341. -Weise, 183'2, Insects Deutschl. 6: 296. --Lefèvre, 1885, Mém. Soc. Sci. Liege, ser. 2, 11: 152. -Jacoby, 1908, Fauna India, Coleopt. 2: 507 (type designated: Cryptocephalus pretiosus Schneider, 1791=Cryptocephalus asclepiadeus Pallas, 1776, from Europe). Chen, 1935, Sinensia 6 (3) : 270; 1940, ibid. 11 (5-6) : 492. -Gressitt \& Kimoto, 1961, Pac. Insects Monogr. 1A: 296. Kimoto, 1964, J. Fac. Agr. Kyushu Univ. $13(2): 241$.

Atymius Gistl, 1837, Systema Insectorum, secundum classes, ordines, genera, species, 1: 61. Monrós\& Bechyné, 1956, Entomol. Arb. Mus. Frey 7: 1127.

\section{Chrysochus pulcher Baly (Figs. 42f-h,43c)}

Chrysochus pulcher Baly, 1864, Descr. new gen. \&spec. Phytoph., 1 (Malay Peninsula; BM); 1867, Trans. Entomol. Soc. Lond., ser. 3, 4: 134, pl. 5, fig. 4 (Malay Peninsula). -Jacoby, 1908, Fauna India, Coleopt. 2: 509 (Malaya).

Chrysochus mouhoti Baly, 1864, J. Entomol. 2: 222 (Sima; BM).--Lefèvre, 1893, Ann. Soc. Entomol. Fr. 1893: 131 (Cochinchina). Jacoby, 1908, Fauna India, Coleopt. 2: 510 (Burma, Siam, Tonkin). New synonymy.

Chrysochus conspectus Lefèvre, 1890, Nouv. Arch. Mus. Paris, ser. 3, 2: 200 (Laos; PARIS) ; 1904, Miss. Pavie Indo-China, ser. 2, 3 (Zool.) :157(Laos). New synonymy.

Chrysochus languei Lef èvre, 1893, Ann. Soc. Entomol. Fr. 62: 132 (Tonkin; PARIS). New synonymy.

Chrysochus massieiLefèvre, 1893, Ann. Soc. Entomol. Fr. 62: 131 (Cambodia :Luang-Prabang ; PARIS). -Gressitt \& Kimoto, 1961, Pac. Insects Monogr. 1A: 298 (Laos, Hainan). New synonymy.

Chryoschus siamensis Jacoby, 1895, Stett. Entomol. Zeit. 56: 67 (Siam ; BM) ; 1908, Fauna India, Coleopt. 2: 509 (Burma, Siam). New synonymy.

Chrysochus adonis Pic, 1928, Mél. Exot. Entomol. 52: 27 (Laos; PARIS). New synonymy. Distribution : Burma, Thailand, Cambodia, Laos, Veitnam, Hainan, Malaya. Subquadrate, oval; coloration of dorsal surfaces variable : (a) purplish 
blue, pronotum golden cupreous, (b) purplish blue to metallic green, pronotum golden green with a round purplish to geenish spot on disc, (c) golden green to greenish blue, pronotum with a round purplish spot on disc, elytron with lateral and apicosutural margin and a broad subbasal transverse band purplish ; length 7.0-9. Om m.

Material EXAmined. THAILAND : Chieng Dao, 1 ex., 15. vi. 1965, K. Morimoto (KU) ; Kanchanaburi, 12 exs., 21. v. 1962 (BANGKHEN). LAOS : Umgeb. Vientiane, 47 exs., iii-vi. 1963; Umgeb. Vanky, 1 ex., 1963; Umgeb. Pakse, 1 ex., 1964 (MUNCHEN) ; Vientiane Prov., Ban Van Eue, 3 exs., 15-31. v. 1965, 1 ex., 31. v. 1966, Native collr (BISHOP).

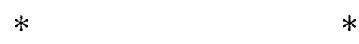

\section{Genus Massiea Lefèvre}

Massiea Lef., 1893, Ann. Soc. Entomol. Fr. 62:132 (type : Massiea cyanipennis Lefèvre, from Cambodia).

According to the original description, this genus was included in Entoce. phalini and related to Dermoxanthus. This genus is not included in the key to genera.

\section{Massiea cyanipennis Lefèvre}

Massiea cyanipennis Lef., 1893, Ann. Soc. Entomol. Fr. 62: 133 (Cambodge :Luang-Prabang). Distribution: Laos.

Oblong, subcylindrical, entirely reddish brown, shining, elytron bluish black ; length $6-6.5 \mathrm{~mm}$.

We could not trace the type location of this species.

The following 3 species are listed under Chrysomelidae, Eumolpinae in Zoological Record, Vol. 61 for the year 1924.

Pseudeumolpus javanus Pic, 1924, Mel. Exot. Entomol. 41: 27 (Annam). Pseudeumolpus substriatus Pic, 1924, ibid. (Java).

Pseudeumolpus binhanus Pic, 1924, ibid. (Tonkin).

These should be listed under Tenebrionidae. This confusion may be caused by the fact that Chrysomelid genus Pseudeumolpus Jacoby, 1893, is a junior homonym of Tenebrionid genus Pseudeumolpus Kraatz, 1880. A new name for Pseudeumolpus Jacoby was given as Eumolpopsis by Jacoby, in 1894. 


\section{References}

Berlioz, J. 1917. Contribution à l'Etude de la Faune Indo-Chinoise. Coléoptères Eumolpides recuellies par Mr. R. Vitalis de Salvaza au Laos, Ann. Soc. Entomol. Fr. 86: 307316.

Chen, S. 1935. Study on Chinese Eumolpid beetles. Sinensia 6(3): 221-387.

1940. New genera of Eumolpinae from Oriental Asia. Sinensia 11: 207-212. 1940. Notes on Chinese Eumolpidae. Sinensia 11: 483-528.

Gressitt, J. L. \& S. Kimoto 1960. The Chrysomelidae (Coleopt.) of China and Korea. Part 1. Pac. Insects Monogr. 1A : 1-299.

\& --- 1963. The Chrysomelidae (Coleoptera) of China and Korea. Part 2. Pac. Insects Monogr. 1B:301-1026.

J. A. Rondon \& S. von Breuning. 1970. Cerambycid beetles of Laos. Pac. Insects Monogr. 24: 1-651.

Jacoby, M. 1889. Viaggio di Leonardo Fea in Birmania e Regioni Vicine, 17. List of the Phytophagous Coleoptera obtained by Signor L. Fea Burmah and Tennasserim, with descriptions of new species. Ann. Mus. Civ. Genova, ser. 2, 7: 147-237.

--- 1892. Viaggio di Leonardo Fea in Birmania e Regioni Vicine, 51. Descriptions of the new genera and species of the Phytophagous Coleoptera obtained by Sign. L. Fea in Burma, Ann. Mus. Civ. Genova 32 : 867-999.

Lefèvre, E. 1885. Eumolpidarum hucusque cognitarum catalogus. Mém. Soc. Sci. Liège, ser. 2, 11: $1-172$.

1889. Contributions à la faune Indo-Chinoise, 4. Cryptocephalides, Clytrides et Eumolpides. Ann. Soc. Entomol. Fr., ser. 6, 9: 287-299.

1890. In Pavie, M., Collection d'insectes formée dans l'Indo-Chine, Coleoptera. Clytridae et Eumolpidae. Nouv. Arch. Mus. Paris, ser. 3, 5: 189-202.

1893. Contributions à la faune Indo-Chinoise, 12. Clytrides \& Eumolpides. Ann. Soc. Entomol. Fr. 1893: 111-134.

1904. Famille des Chrysomélides des Clytrines et des Eumolpines. Mission Pavie Indo-China 1879-1895, ser. 2, 3: 146-164, pl. 9. 